\title{
Antibiotic resistance reservoirs: the cases of sponge and human gut microbiota
}

Dennis Versluis 


\section{Thesis committee}

\section{Promotor}

Prof. Dr. H. Smidt

Personal chair at the Laboratory of Microbiology

Wageningen University

\section{Co-promotors}

Dr. M.W.J. van Passel

Senior project-coordinator

Institute for Public Health and the Environment (RIVM), Bilthoven

Dr. D. Sipkema

Assistant professor, Laboratory of Microbiology

Wageningen University

\section{Other members}

Prof. Dr. J.A.G.M. de Visser, Wageningen University

Prof. Dr. M.J.M. Bonten, University Medical Center Utrecht

Prof. Dr. P.J. Schupp, Institute of Chemistry and Biology of the Marine Environment, Wilhelmshaven, Germany

Dr. B.E. Dutilh, Utrecht University

This research was conducted under the auspices of the Graduate School VLAG (Advanced studies in Food Technology, Agrobiotechnology, Nutrition and Health Sciences) 


\section{Antibiotic resistance reservoirs: the cases of sponge and human gut microbiota}

Dennis Versluis

\section{Thesis}

submitted in fulfilment of the requirement for the degree of doctor at Wageningen University

by the authority of the Rector Magnificus

Prof. Dr. A.P.J. Mol,

in the presence of the

Thesis Committee appointed by the Academic Board

to be defended in public

on Monday 21 November 2016

at 11 a.m. in the Aula. 
Dennis Versluis

Antibiotic resistance reservoirs: the cases of sponge and human gut microbiota, 197 pages.

PhD thesis, Wageningen University, Wageningen, NL (2016)

With references, with summaries in Dutch and English

ISBN 978-94-6257-905-7

DOI 10.18174/388428 
This thesis is dedicated to my parents 



\section{Table of contents}

Chapter 1. General Introduction \& Thesis Outline 1

Chapter 2. Mining microbial metatranscriptomes for expression of 19 antibiotic resistance genes under natural conditions

Chapter 3. High throughput recovery of previously uncultured bacterial genera from three Mediterranean sponges

Chapter 4. Sponge microbiota are a reservoir of functional antibiotic resistance genes

Chapter 5. Comparative genomics of the marine genus Pseudovibrio 93 highlights symbiotic capacities of versatile bacteria

Chapter 6. High throughput cultivation-based screening on the MicroDish platform allows targeted isolation of antibiotic resistant human gut bacteria

Chapter 7. General Discussion

References

Summary 

General Introduction \&

Thesis Outline 


\section{Thesis aim}

Antibiotic resistance poses a serious threat to human health to the extent that options for the treatment of infections by antibiotic-resistant bacteria are becoming increasingly limited. The first step in containing this problem would be to thoroughly understand antibiotic resistance with respect to its development, persistence and expansion. Hence, the aim of this thesis is to study aspects of antibiotic resistance that are still poorly understood, with a focus on environmental antibiotic resistance. One important aspect lies in the identification of biological niches and bacterial taxa that are important reservoirs of antibiotic resistance. Furthermore, it is important to comprehend to what extent resistance is exchanged between reservoirs, and by what mechanisms resistance exchange occurs. The most relevant issue in this context is the role of the environmental resistome in the occurrence of resistance genes in microorganisms causing nosocomial infections. In this thesis, these topics are addressed with a particular focus on the human gut microbiota, the sponge microbiota, and the marine genus Pseudovibrio. We specifically aimed to identify novel antibiotic resistance reservoir species and to investigate the presence of resistance determinants in their respective genomes. The research described in this thesis expands our knowledge and thereby contributes to finding novel leads to combat the emergence of antibiotic resistance.

This Introduction Chapter gives context to the research chapters by reviewing relevant scientific literature. The first paragraph provides a historical perspective regarding antibiotic discovery and emerging resistance. The second paragraph describes the human pathogens that currently pose a major health threat, and the text also explains why research into environmental resistance is crucial. This is followed by introduction of relevant topics, including horizontal gene transfer, cellular targets of antibiotics, antibiotic resistance mechanisms, and methods to study antibiotic resistance. Subsequently there is a short overview of literature discussing environmental resistance reservoirs followed by a more in-depth description of the current knowledge with respect to microbial communities associated with sponges and the human gut. Furthermore, the marine bacterial genus Pseudovibrio is introduced in more detail as potential reservoir of antibiotic resistance. Finally, an outline is given of the remaining chapters in this thesis. 


\section{Antibiotics - discovery and emerging resistance}

Since their introduction in the 1930s, antibiotics have played a central role in modern health- and veterinary care, where they act by killing or arresting growth of bacteria while the cells of the human or animal host remain unharmed. In the pre-antibiotic era, when treatment of bacterial infections included rinsing with alcohol, maggot debridement therapy [1, 2], patient isolation [3] and treatment with chemical compounds, these infections were life-threatening, and treatment was often not successful. The first clinically applied antibiotics were the sulfonamides in 1935, penicillin in 1941, and streptomycin in 1943 [4]. Following the introduction of these antibiotics, bacterial infection-related mortality (e.g. bacterial pneumoniae and tuberculosis) dropped sharply [5]. The 1950s and 1960s marked the Golden Age of antibiotic discovery in which most important classes of antibiotics in use today were discovered. In subsequent decades, natural antibiotics from most important classes were optimized for potency, and their properties were improved by modification of the chemical structure [6]. Nowadays, physicians do not only rely on antibiotics to treat infections, but also to severely reduce risks of infections when performing complicated operations. In addition, antibiotics are administered prophylactically to patients that are admitted to intensive care units [7].

Already during the Golden Age of antimicrobial discovery, antibiotic resistant strains were being encountered [4]. However, initial reports about horizontal gene transfer (HGT) were still regarded sceptically [8], that is, resistance was considered to be limited to the specific strain in which it was detected. In subsequent decades, more pathogens developed multidrug resistance, and scientists started to appreciate the high impact of HGT, and specifically conjugation, in resistance dissemination [4]. This emergence of antibiotic resistance was mainly attributed to high antibiotic use and resulted in some once-treatable infections becoming difficult or impossible to cure [9]. Not only did resistance emerge, but also fewer new antibiotics entered the market that could complement the current repertoire [10]. Outbreaks of strains resistant to last resort antibiotics such as the Klebsiella pneumoniae outbreak in the Maasstad hospital in Rotterdam started occurring more frequently [11-13]. Today, antibiotic resistance is considered a global threat that presents a great social and economic burden on societies. The CDC estimated that in the United States alone in 2013 two million infections and 23,000 deaths were attributable to antibiotic 
resistance [14]. As a response to these concerning developments, policy makers are now increasingly taking action. On March $27^{\text {th }}, 2015$, the Obama administration launched an action plan for combating antibiotic-resistant bacteria, and on February $4^{\text {th }}, 2016$, it was announced that the Dutch Ministry of Health increased the budget for research on antibiotic resistance by $€ 16$ million [15].

\section{The antibiotic resistance crisis and prospective research}

The rise in multidrug-resistant bacterial infections by major pathogens has led the WHO to declare that we are currently in a global antibiotic resistance crisis with potentially devastating costs to human society [16]. The Gram-positive pathogen posing the greatest threat to human health is methicillin-resistant Staphylococcus aureus, which in the USA alone is attributed $\sim 11,000$ deaths per year [17, 18]. Another group of Gram-positive pathogens representing a major therapeutic challenge are the Vancomycin-resistant enterococci [18-20]. Among Gram-negative pathogens, infections by extended-spectrum $\beta$-lactamase-producing and/or carbapenem-resistant Enterobacteriaceae, (mostly Klebsiella pneumoniae) [17, 18, 21], multidrug-resistant (MDR) Streptococcus pneumoniae [17, 18], MDR Mycobacterium tuberculosis [17, 22], MDR Pseudomonas aeruginosa [18, 23] and MDR Acinetobacter spp. [18] resulted in the highest mortality rates. MDR pathogens are a global concern because highly mobile individuals can lead to epidemic pathogenic spread $[24,25]$. At present, transmissible resistance to nearly all clinical antibiotics has been found in human pathogens indicating the importance of understanding resistance dissemination [18]. For example, the first mobilizable gene conferring resistance to colistin (mcr-1), which is considered to be a last resort antibiotic [26], was found recently in E. coli strain SHP45 that was isolated from a pig [27]. Ever since the description of $m c r-1$, animal-to-human transmission of the gene has already been detected in China [28], Denmark [29], Laos [30] and Thailand [28, 30]. Hence, even though carbapenem-resistant Enterobacteriaceae can still be treated with colistin, there is now the imminent threat that these bacteria acquire the mcr-1 gene, resulting in pan-resistant infections becoming truly untreatable [27]. Antibiotic stewardship [31, 32], prudent application of antibiotics in food animals [33, 34], and application of novel antibiotics [35, 36] are important strategies to curb the emergence of antibiotic resistance in pathogens. However, in order to develop effective strategies for preventing and controlling resistance spread, it is also 
essential to understand in-depth to what extent, and via which mechanisms, both human-associated and environmental bacteria contribute to resistance dissemination. Knowledge with regards to the role of environmental bacteria in acquisition and spread of antibiotic resistance is still very limited [37-39] even though the majority of resistance genes are expected to have originated from non-human sources [40]. The notion that environmental bacteria are highly relevant is further supported by them being a rich and assorted reservoir of resistance genes that may be heterologously expressed in human pathogens [41-44]. As such, there is the need to identify important relevant resistance reservoirs (e.g. bacteria in animals, soil or aquatic environments), and to assess the transferability of environmental resistance to human pathogens. In specific, the dynamics of resistance gene dissemination, the mechanisms of resistance gene transfer, and the conditions that promote resistance transfer need to be investigated $[40,45]$. Responding to these major research challenges, and with the ambition to generate novel insights into these processes, the Evolution and Transfer of Antibiotic Resistance (EvoTAR) project was initiated within the Seventh Framework Programme of the European Commission, including the research described in this thesis.

\section{Horizontal gene transfer}

HGT is considered the main mode of inheritance by which antibiotic resistance genes are disseminated and by which pathogenic bacteria develop resistance to clinical antibiotics [46-48]. HGT is defined as exchange of genetic material between organisms beyond transfer through procreation. In bacteria, the three mechanisms through which HGT can occur are conjugation, transformation and transduction [49, 50]. Conjugation is the transfer of genetic material from the donor to the recipient through a pilus. Transformation is incorporation of exogenous DNA taken up directly from the environment. Transduction is the delivery of exogenous genetic material into the cell by a phage. HGT mechanisms principally exchange resistance genes located on plasmids whereas lateral exchange of chromosomally located resistance genes occurs less frequently [51,52]. Aggregation of resistance genes on plasmids allows for bacteria to become simultaneously resistant to multiple antibiotics from a single DNA transfer event $[53,54]$. Analysis of fully sequenced bacterial genomes revealed that a substantial fraction of open reading frames have been horizontally transferred indicating the importance of HGT in bacterial diversification $[55,56]$. The rate at 
which genetic exchange between bacteria occurs is limited by their genetic dissimilarity and spatial distribution. However, even though HGT events predominantly occur between closely related species, bacterial recombination can extend across bacterial phyla and even across the domains of life [57-59]. Therefore, any resistance gene harboured by a bacterium can potentially provide resistance to another bacterium that was previously susceptible as long as the cellular machinery is compatible. The horizontal spread of resistance-harbouring plasmids through conjugation is the most important mechanism by which pathogenic bacteria in the clinic acquire resistance $[51,60]$.

\section{Antibiotic targets and antibiotic resistance}

Antibiotics kill (bactericidal drugs) or inhibit (bacteriostatic drugs) growth of bacteria by disrupting essential processes or cellular components. Nearly all antibiotics target one of the following processes: DNA replication, nucleotide biosynthesis, transcription, cell wall synthesis, cell wall stability or protein production [61]. In 2010, the classes of antibiotics that were administered to patients most often were the $\beta$ lactams (e.g. ampicillin), macrolides (e.g. erythromycin), fluoroquinolones (e.g. ciprofloxacin), tetracyclines (e.g. tetracycline), amphenicols (e.g. chloramphenicol), aminoglycosides (e.g. kanamycin), and trimethoprim [62]. B-lactams by definition contain a four-membered $\beta$-lactam ring in their molecular structure and block crosslinking of peptidoglycan layers of bacterial cell walls. B-lactams are comprised of the subclasses penicillins, cephalosporins, monobactams, and carbapenems [63]. Tetracyclines and aminoglycosides inhibit translation by targeting the $30 \mathrm{~S}$ ribosome while macrolides target the $50 \mathrm{~S}$ ribosome [61]. Fluoroquinolones impair DNA replication by interfering with DNA supercoiling [64]. Trimethoprim inhibits synthesis of nucleotides by binding to dihydrofolate reductase [65]. Said antibiotics are effective against both Gram-positive and Gram-negative bacteria. However, some antibiotics can be classified based on whether they primarily target Gram-positive or Gramnegative bacteria. Polymyxins (e.g. colistin), which exclusively target Gram-negative bacteria, disturb the cell membrane resulting in leakage of cell contents and eventually cell death [66]. Glycopeptides (e.g. vancomycin) and oxazolidinones (e.g. linezolid) predominantly affect Gram-positive bacteria by inhibition of cell wall crosslinking and protein synthesis, respectively $[44,67,68]$. 
Bacterial resistance to an antibiotic can be described as intrinsic or as acquired. In the case of intrinsic resistance, the bacterium naturally does not possess the target site for the drug. Alternatively, low permeability of the cell wall to the drug may prevent the drug from reaching its target. In the case of acquired resistance the bacterium is naturally susceptible, and resistance can be acquired by four general mechanisms: (i) modification or inactivation of the antibiotic, (ii) reduction of the binding capacity of the antibiotics by target site alteration, (iii) modification of a metabolic pathway to circumvent the effects, and (iv) reduction of the intracellular antibiotic concentration by efflux or decreased membrane permeability [69, 70]. A bacterium can acquire resistance either by mutations in the bacterial chromosome, or by procurement of an exogenous resistance gene. Resistance genes are often located on mobile genetic elements that facilitate HGT such as plasmids, transposons and integrons. Figure 1.1 shows the mechanisms of acquired resistance to the most important classes of antibiotics.

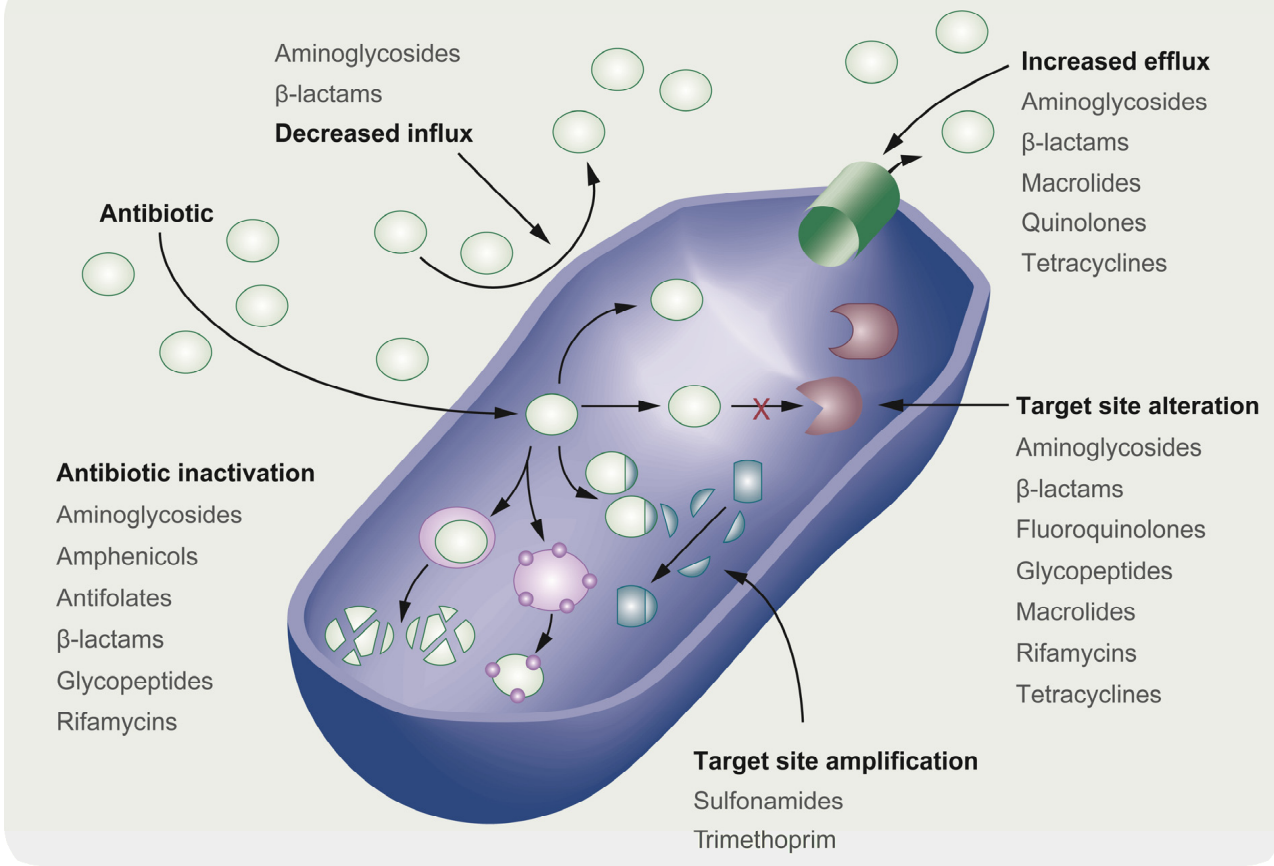

Figure 1.1 Schematic representation of the mechanisms by which bacteria can become antibiotic resistant. This figure was adapted from [69]. 


\section{Methods to study antibiotic resistance}

Methods to study antibiotic resistance can be separated into those that are uniquely suitable to study resistance in bacteria in pure culture, and to those that are suitable to study resistance in bacterial communities but may also be applied to study resistance in bacteria in pure culture.

The main advantages of studying bacteria in pure culture are that identified resistance genes can be assigned to a single bacterium rather than to a community, and that the bacteria can be characterized. Characterization includes performing resistance profiling, studying how antibiotics affect cell physiology and morphology, and investigating if bacteria themselves produce antibiotics. Transposon mutagenesis can be used to identify the genes conferring resistance if the cellular machinery of the bacterium in question is compatible with this method [7]. Alternatively, the bacterial genome can be sequenced, and putative resistance genes can be identified based on similarity to known resistance genes [71]. In vitro evolution experiments can be performed to investigate how antibiotic resistance evolves under different experimental conditions [72]. Pure bacterial cultures also permit bacteriumto-bacterium HGT (e.g. conjugation) experiments [73].

Methods for studying antibiotic resistance at the community level can be separated into three approaches that each study a different biological information tier, namely (i) metagenomics; the study of collective DNA, (ii) metatranscriptomics; the study of collective RNA, and (iii) metaproteomics; the study of collective proteins (Figure 1.2). All three approaches can be applied culture-independently. The most commonly used approach to study the bacterial resistome, i.e. the collection of all resistance genes in a bacterial community [74], is metagenomics. Metagenomics in the context of the resistome involves the deep sequencing of community DNA, and subsequent mapping of the (assembled) reads to an antibiotic resistance gene database [69]. Occasionally researchers apply "targeted" metagenomics, which uses community DNA for microarray hybridization $[75,76]$ or (quantitative) PCR [77, 78]; the disadvantage being that the number of resistance genes that can be detected is limited to the size of the experiment and similarity to known resistance genes. Functional metagenomics involves the expression of community DNA in a surrogate host (usually Escherichia coli). For this purpose, fragmented community DNA is ligated into a vector, either as $<5 \mathrm{kbp}$ fragments into a routine vector, or as $\sim 40 \mathrm{kbp}$ 
fragments into a fosmid vector. Subsequently, transformation of $E$. coli with the ligation mixture yields a library that is then plated out on media containing antibiotics. Resistant clones are picked and their inserts, which confer resistance, are sequenced $[69,79,80]$. Functional metagenomics is more time- and resource-consuming than the previously mentioned methods but has the advantage that identified resistance genes are confirmed to be functional, and that novel resistance genes (i.e. resistance genes with no significant similarity to those already deposited in the resistance gene databases) can be identified. Metatranscriptomic and metaproteomic approaches have not yet been used as dedicated tools to study antibiotic resistance though these approaches have been used to selectively draw conclusions about active resistance in environmental samples [81, 82]. The research in Chapter 2 of this thesis applies metatranscriptomics to study antibiotic resistance in a range of ecological niches.

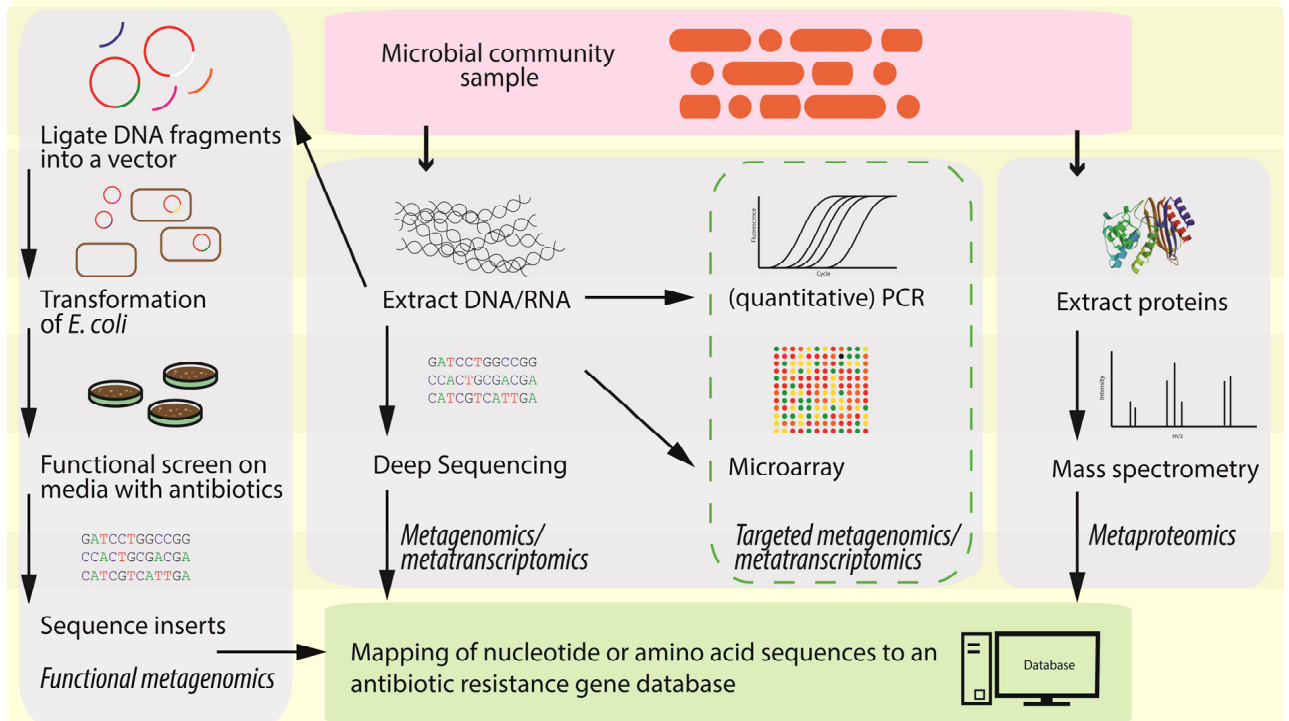

Figure 1.2 Different methodological approaches for the study of the resistome of bacterial communities.

\section{Microbial cultivation methods}

It is generally accepted that only a small fraction $(\sim 1 \%)$ of the total bacterial diversity can be readily cultivated in the laboratory - often referred to as the 'great plate count anomaly' [83, 84]. Historically, isolation of uncultured bacterial species was mainly achieved by application of diverse growth media. However, in recent years, this strategy has had reduced success, especially regarding well-studied biological niches such as the human gut microbiota. For example, it took as much as 88 culture 
conditions and 12,700 different picked colonies to only isolate 11 new bacterial species from a human faecal sample [85]. On the other hand, in a recent study $90 \%$ of species-level bacterial abundance in 6 human faecal samples was cultivated, thereby challenging the notion that the isolation of novel gut bacteria by classical cultivation methods has plateaued [86]. Bacteria will resist in vitro growth if essential aspects of their natural environment are not properly replicated. Essential aspects for bacterial growth can be divided in abiotic (including, but not restricted to, $\mathrm{pH}$, nutrients, temperature, oxygen concentration and light intensity) and biotic (i.e. microbe-microbe and host-microbe interactions) factors, where biotic factors are typically much more difficult to mimic in vitro. Bacteria depending on other organisms to remove toxic end products or to supply essential growth factors [87] are examples of biotic factors.

Nowadays, an increasing number of innovative culturing techniques are being applied, either involving implementation of non-traditional in vitro conditions - at least in the initial stages of bacterial isolation - or involving cultivation conditions specifically designed to isolate a target bacterium. Non-traditional methods include co-culturing and community culturing, which have already been successfully applied to cultivate previously uncultured bacteria [88]. Another novel cultivation method is the application of multi-well micro culture chips such as the MicroDish Culture Chip (MDCC) [89]. MDCCs allow bacterial colonies to grow at an unprecedented density on a porous aluminium oxide support through which small molecules (i.e. nutrients and growth factors) can diffuse (Figure 1.3). Bacterial cultivation of novel species has also been achieved by application of devices with $>250$ small growth chambers. Growth chambers with liquid media were inoculated with on average one bacterial cell from the sample of interest. Subsequently, the chambers, which have semipermeable membranes that only allow passing of small molecules, were placed in the natural environment of the bacteria [35, 90,91]. These are only a few examples of innovative cultivation methods; however, more comprehensive reports can be found in various excellent review papers on this topic [87, 92, 93]. Isolation of uncultured bacterial species with a targeted approach requires at least some knowledge with respect to its metabolic requirements and necessary growth conditions. Predictions about these aspects may be derived from the full genome sequence of the target bacterium, or from a (partial) genome reconstructed from metagenomic data [94]. 
Even though this approach has promise, so far very few studies have been published that use genomic information to achieve cultivation [95, 96].

(A)

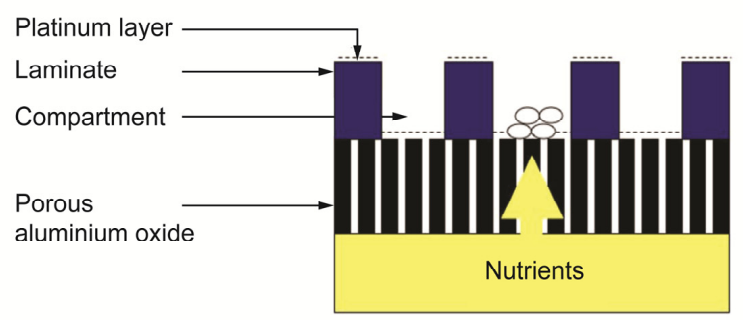

(B)

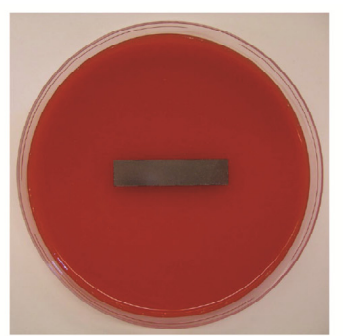

Figure 1.3 MicroDish Cultivation Chips (MDCCs) consist of a porous aluminium oxide support with on top an acrylic polymer laminate containing discrete round compartments. MDCCs are available with up to 180,000 compartments allowing for high-throughput cultivation of bacteria. (A) Cross-sectional diagram of a small part of the MDCC. (B) Photograph of an MDCC placed on top of blood agar. The pictures are from [89].

\section{Environmental reservoirs of antibiotic resistance}

Resistance genes have been detected in a broad range of biological niches associated with complex microbial communities [97]. Resistance genes in these socalled reservoirs can theoretically spread into other environments and previously non-resistant microbial populations, including human pathogens. Soil is the most recognized reservoir, but also activated sludge [98], wastewater effluent [99], animals [100, 101], oceans [102], lakes [97] and rivers [103] were found to harbour diverse clinically relevant resistance genes (Figure 1.4). Studies have shown that microbial resistomes cluster by ecological niche, and even within an ecological niche independent microbial communities appear to contain particular subsets of resistance genes [99, 104-106]. The fact that antibiotic resistance functions are largely constrained by ecology suggests that niches should be evaluated independently as potential resistance reservoirs. It is still unknown which niches are the greatest contributors to antibiotic resistance in human pathogens [105, 107]. Functional antibiotic resistance genes have been identified in isolated caves and permafrost soil indicating that resistant genes are ancient and predate the era of application of antibiotics in human health care [108, 109]. Despite the high diversity of resistance genes in the miscellaneous environmental niches, only few cases have been documented involving transfer of environmental resistance genes to human pathogens. For example, the CTX-M $\beta$-lactamases that have been emerging in Gram-negative pathogens [110] are known to have originated from environmental 
Kluyvera spp. [111]. As another example, the quinolone resistance-conferring qnrA gene was shown to have originated from Shewanella algae residing in marine and fresh water [112, 113]. Furthermore, in soil, aminoglycoside, tetracycline, sulfonamide, amphenicol and $\beta$-lactam resistance genes were identified that shared $100 \%$ nucleotide identity with genes in known pathogens [43]. A perfect nucleotide identity either indicates that soil bacteria are a direct resistance source that can be harnessed by human pathogens, or it indicates that human pathogens or their genes have spread into other environments. To sum up, even though it is clear that environmental resistance has a clinical impact, little is yet known with respect to resistance exchange mechanisms and frequencies.

One ecological niche that has been little-studied with regards to antibiotic resistance is the marine environment [102]. Given its microbial diversity, putatively comprising $\sim 90 \%$ of total prokaryotic biomass on earth [114, 115], this niche is expected to harbour diverse antibiotic resistance functions.

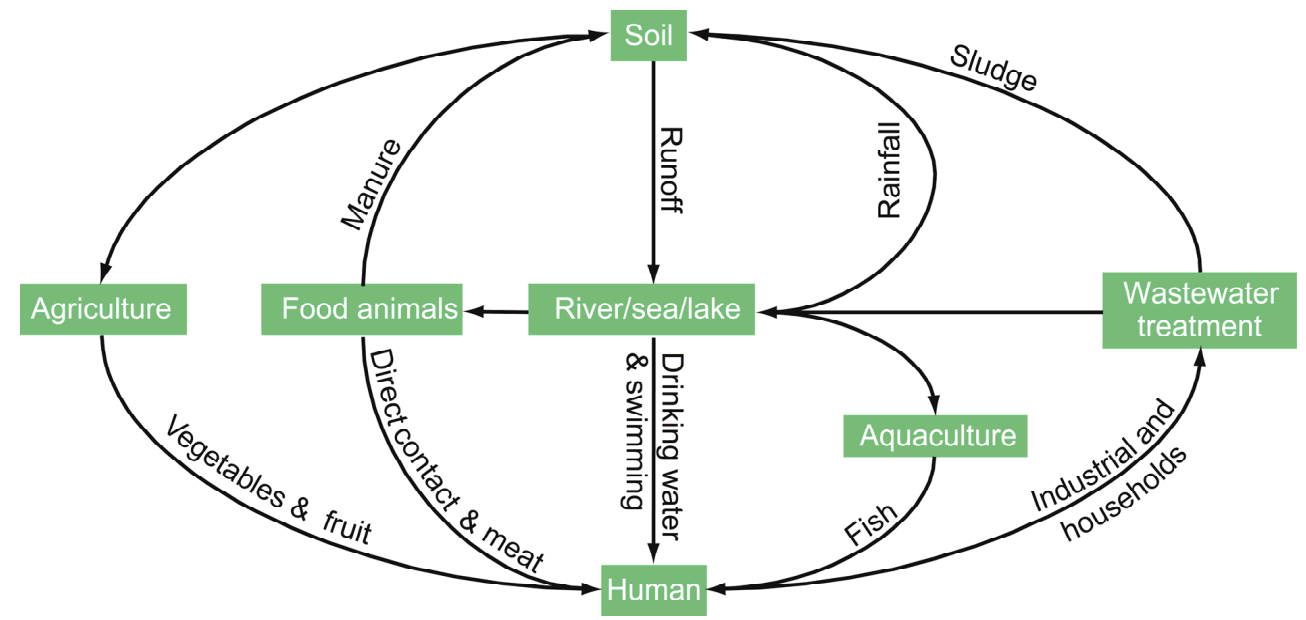

Figure 1.4 Potential routes of resistance gene dissemination. The arrows indicate possible routes through which antibiotic resistance can be disseminated. This figure was adapted from [69].

\section{Sponge microbiota and antibiotic resistance}

Sponges arose 600-700 million years ago and since that time have been consistent members of benthic communities in the aquatic environment [116]. Their diet consists of plankton and organic debris that they filter from surrounding water. Although sponges actively feed on microorganisms, they frequently simultaneously harbour dense and highly diverse microbial communities that co-exist with the host in a symbiotic relationship $[117,118]$. Various roles have been suggested by which the 
associated microbiota may benefit the sponge host, including in anti-predation [119], antifouling [119], nitrogen fixation [120], nitrification [121], carbon fixation [122] and defense against microbial pathogens [123].

Sponges can be divided according to the bacterial cell density in their tissues where they either have a bacterial concentration within the range of that in surrounding sea water (low-microbial-abundance sponges), or the bacterial concentration is increased by 2-4 orders of magnitude (high-microbial-abundance sponges) [124]. In high-microbial-abundance sponges, the microbial community can comprise as much as $40 \%$ of the sponge volume [125]. A meta-analysis of publicly available $16 \mathrm{~S}$ rRNA gene sequences of sponge-associated bacteria showed that, overall, Proteobacteria, Actinobacteria, Bacteroidetes, Chloroflexi and Firmicutes are the dominant bacterial phyla [123]. However, there is great variation in spongeassociated microbiota, and remarkably, bacterial communities seem to be largely host species-specific, with only few bacterial taxa shared between sponge species [126-128]. Only a small fraction of sponge-associated bacteria is currently amenable to cultivation [125], and this mainly includes bacteria from the phyla Proteobacteria, Firmicutes, Bacteroidetes and Actinobacteria [129-131].

Sponges, and in particular their associated microbes [132], are recognized as the richest source of bioactive compounds in the marine environment [133]. Accordingly, over the last decades, sponges have been receiving increasing interest from pharmaceutical and biotechnological industries as sources of drugs against e.g. cancer, viruses, inflammations and infections [134, 135]. Mining of unexplored sponge species has shown to consistently yield new bioactive compounds with novel chemical structures [136]. However, the discovery of novel drug leads is hampered by only a small fraction of sponge-associated bacteria being currently cultivable in the laboratory. Furthermore, logistical obstacles often preclude collecting large quantities of sponge material to be used in experiments [137]. In the natural environment, these bioactive compounds often provide important defensive capabilities to a static organism that is otherwise easily preyed upon [138]. Given that some sponges contain complex microbial communities that can produce diverse antimicrobials, it could be speculated that sponge holobionts also contain diverse antibiotic resistance genes. As a consequence, it is tempting to speculate that sponges might be a reservoir of yet unknown resistance genes that may be harnessed by bacteria in other environments. So far, research on functional antibiotic resistance genes in 
sponge microbiota has been limited to a Bacillus sp. isolated from the sponge Haliclona simulans. The Bacillus sp. was found to contain two small plasmids, one of which harboured the tet $(\mathrm{L})$ tetracycline resistance gene [139] whereas the other harboured the $\operatorname{erm}(\mathrm{T})$ erythromycin resistance gene [140]. Tetracycline and erythromycin antibiotics are both commonly used in the clinics [141], and as such, these findings indicate that sponges are potential sources of emerging resistance. $\mathrm{A}$ recent study detected the mecA, mupA, tetL and $q n r B$ resistance genes in the sponge Petromica citrina by PCR with gene-specific primers [142]. With the uncovered bacterial diversity in these metazoans it is likely that both a large diversity of antibiotics as well as their concomitant resistance genes may be mapped.

\section{The marine bacterial genus Pseudovibrio}

Members of the a-proteobacterial genus Pseudovibrio have been isolated exclusively from marine habitats, that is, those habitats that depend on salt water from the sea. The first published type species was Pseudovibrio denitrificans in 2004 [143], and since then the genus has been expanded to include six species [144, 145]. Interestingly, studies have shown that Pseudovibrio spp. comprise most of the cultivable fraction of various sponge species [146-148], while never simultaneously being isolated from nor detected in surrounding sea water [149, 150]. This led to the hypothesis that Pseudovibrio spp. have a symbiotic relationship with sponges [151]. The finding that Pseudovibrio was transferred via the germline in the sponge Mycale laxissima [150] strengthened this hypothesis. Besides sponges, Pseudovibrio spp. have been isolated from ascidians [152], a flatworm [144], tunicates [153, 154] and corals $[155,156]$. Even though Pseudovibrio spp. appear to be enriched in various marine animals, they have also been isolated from sea water [143, 157]. Members of this genus have received high scientific interest as a source of novel clinically relevant bioactive compounds with potent broad-spectrum antimicrobial activity [158, 159]. To date, three antimicrobial compounds have been identified. Pseudovibrio ascidiaceicola $\mathrm{F} 423^{\top}$ was found to produce phenazine [160], the coral-associated Pseudovibrio strain PaH3.28 was found to produce the peptide pseudovibrocin [156], and the algae-associated Pseudovibrio sp. D323 was found to produce tropodithietic acid [161]. Numerous scientific articles speculate on the symbiotic role that Pseudovibrio spp. may have in sponges. Roles that have been suggested include functions in nutrient uptake [149], denitrification [162, 163], sponge health [164, 165], 
modulation of the host immune system [151], and host defense [161, 166]. Although it is now well-established that microbes are important for sponge health, the exact mechanisms behind the beneficial interactions remain to be elucidated [123].

\section{The human gut microbiota and antibiotic resistance}

The human digestive tract contains a community of microorganisms, collectively referred to as the human gut microbiota. The term gut microbiome is less defined but almost always includes the genes and genomes of the gut microbiota [167]. Anoxic conditions in the gut dictate that gut residents are predominantly anaerobic or facultatively anaerobic [168]. The number of microorganisms in the human digestive tract is estimated to have the same order of magnitude as the number of human body cells (i.e. $3-4 \times 10^{13}$ ) [169], and is dominated by the bacterial phyla Firmicutes, Bacteroidetes, Proteobacteria, Actinobacteria [170]. Bacteria from other bacterial phyla, fungi, viruses, and archaea are also present but at a lower relative abundance $[170,171]$. A stable gut microbiota is essential for the health of the human host with beneficial roles in e.g. colonization resistance, nutrition, metabolism of non-digestible dietary compounds and xenobiotics, proliferation and differentiation of intestinal mucosal epithelial cells, and homeostasis of the immune system [172-175]. Administration of antibiotics can result in gut microbiota dysbiosis with antibioticinduced alterations potentially persisting years after treatment [176]. Besides negatively affecting the health of the human host, antibiotic treatment will also enrich the gut microbiota with resistant bacteria and resistance genes [177]. In fact, once an antibiotic resistance determinant has been introduced into the gut microbiota, it will generally continue to persist at some low level, and reintroduction of the antibiotic will reselect resistant strains despite months or even years of non-use [178]. As a result, administration of antibiotics will lead to a greater resistance reservoir in the gut that may be harnessed by pathogenic bacteria. This causal relation was also observed in a study that assessed faecal metagenomes of 252 individuals to show that the resistome correlated more strongly with antibiotics that were on the market longer [104]. It is important to note that also in the gut microbiota from isolated human populations (i.e. remote populations that are unexposed to the effects of anthropogenic antibiotic pressure) resistance genes have been detected [179-181], suggesting either the presence of ancestral resistance genes, or the spread of resistance genes to remote areas [177]. 
The high density and diversity of bacteria in the gut microbiota provides ample opportunity for exchange of resistance genes between different taxa to occur. Resistance gene transfer from commensal gut bacteria to opportunistic pathogens appears to be relatively rare [80]. More often, gut commensals appear to function as reservoir-species of resistance genes that may be acquired by incoming pathogens. For example, Bacteroidetes have been suggested to be an important resistance reservoir as they can acquire DNA from a wide range of bacterial taxa [182]. The carbapenem-resistance-conferring $\beta$-lactamase NDM-1 was first detected in the opportunistic pathogen Klebsiella pneumoniae in 2008, but is now hosted by various gut commensals belonging to the Enterobacteriaceae [183]. This is alarming because carbapenems are often the last resort in the treatment of infections by Gram-negative pathogens [184]. Furthermore, Sommer and colleagues [185] identified functional resistance genes in cultured aerobic isolates, where nearly half of these genes was $100 \%$ identical to resistance genes harboured by major human pathogens. These results, and those of other studies [186-188], support the notion that the gut microbiota is not merely a resistance reservoir but also a mixing vessel that contributes to the generation of (multi-)antibiotic resistant pathogens.

\section{Thesis outline}

Chapter 2 studies the expression of resistance genes in natural environments i.e. those environments that have not been subjected to anthropogenic antibiotic pressure in the recent past. In addition, local antibiotic biosynthesis is assessed by investigating the expression of relevant secondary metabolite gene clusters. The ecological niches that were investigated are sea bacterioplankton, a marine sponge, forest soil, sub-seafloor sediment and the gut microbiota of humans, mice and pigs. In all studied ecological niches resistance genes were found to be expressed, albeit with niche-specific differences. Resistance gene expression could not be linked to antibiotic biosynthesis.

Chapter 3 describes a high-throughput cultivation experiment in which the cultivable bacterial fraction was studied from the Mediterranean sponges Aplysina aerophoba, Corticium candelabrum and Petrosia ficiformis. Bacterial populations growing on different agar media were analysed by $16 \mathrm{~S}$ ribosomal RNA (rRNA) gene amplicon sequencing. The results showed that previously uncultured bacteria can be cultivated by conventional plating. Notably, many species were cultivated from the 
phylum Actinobacteria, which are important sources of novel bioactive compounds. Thirty-one different bacterial strains were cultivated up to pure culture from agar media containing (combinations of) antibiotics, and further phenotypically characterized.

In Chapter 4 the presence and diversity of functional resistance genes in the sponges $A$. aerophoba, $C$. candelabrum and $P$. ficiformis is investigated. Small-insert libraries were prepared in $E$. coli with sponge holobiont DNA, as well as with pooled DNA from the 31 bacterial isolates obtained from these sponges as described in Chapter 3. The study revealed diverse and/or novel resistance genes that conferred resistance to ampicillin, D-cycloserine, gentamicin, amikacin, trimethoprim, chloramphenicol and rifampicin.

Chapter $\mathbf{5}$ entails a comparative genomics study of the marine bacterial genus Pseudovibrio. Thirty-one Pseudovibrio genomes were analysed, including those of five type strains, as well as 18 isolates obtained in the cultivation experiment described in Chapter 3. The phylogeny of the Pseudovibrio strains was resolved, and features regarding symbiosis, secondary metabolite biosynthesis and antibiotic resistance were studied. Various findings support the hypothesis that Pseudovibrio spp. are symbionts of sponges.

Chapter 6 describes a cultivation experiment that aims to investigate yet unexplored bacterial taxa of the human gut as reservoirs of antibiotic resistance. Therefore, faecal samples from 20 intensive care patients that received antibiotic treatment were inoculated anaerobically on a porous aluminium oxide chip on top of agar media. Subsequently, the bacterial growth communities were analysed by $16 \mathrm{~S}$ rRNA gene amplicon sequencing. We detected growth of $>10$ novel species at $\geq 2 \%$ relative abundance. A multi-drug resistant Ruminococcaceae sp. was obtained by targeted isolation.

Chapter 7 summarizes and discusses the work described in this thesis. The results are related to current knowledge in the fields of antibiotic resistance and bacterial cultivation. The major challenges that should be addressed as part of future research are discussed. 



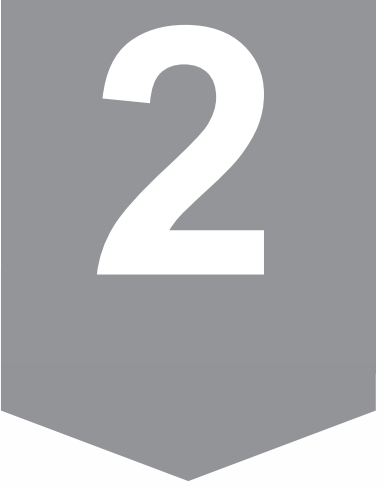

Mining microbial metatranscriptomes for expression of antibiotic resistance genes under natural conditions

Dennis Versluis, Marco Maria D’Andrea, Javier Ramiro Garcia, Milkha M. Leimena, Floor Hugenholtz, Jing Zhang, Başak Öztürk, Lotta Nylund, Detmer Sipkema, Willem van Schaik, Willem M. de Vos, Michiel Kleerebezem, Hauke Smidt, Mark W.J. van Passel 


\section{Abstract}

Antibiotic resistance genes are found in a broad range of ecological niches associated with complex microbiota. Here we investigated if resistance genes are not only present, but also transcribed under natural conditions. Furthermore, we examined the potential for antibiotic production by assessing the expression of associated secondary metabolite biosynthesis gene clusters. Metatranscriptome datasets from intestinal microbiota of four human adults, one human infant, 15 mice and six pigs, of which only the latter have received antibiotics prior to the study, as well as from sea bacterioplankton, a marine sponge, forest soil and sub-seafloor sediment, were investigated. We found that resistance genes are expressed in all studied ecological niches, albeit with niche-specific differences in relative expression levels and diversity of transcripts. For example, in mouse and human infant microbiota predominantly tetracycline resistance genes were expressed while in human adult microbiota the spectrum of expressed genes was more diverse, and also included $\beta$-lactam, aminoglycoside and macrolide resistance genes. Resistance gene expression could result from the presence of natural antibiotics in the environment, although we could not link it to expression of corresponding secondary metabolites biosynthesis clusters. Alternatively, resistance gene expression could be constitutive, or these genes serve alternative roles besides antibiotic resistance. 


\section{Introduction}

Antibiotic resistance genes are ubiquitous in bacterial communities in various ecological niches [97]. Metagenomic studies have revealed the presence of resistance determinants in environments as different as soil, gut, sea water, caves and glaciers [108, 109, 185, 189-192]. Bacterial communities in these and other ecological niches contain particular subsets of antibiotic resistance determinants, and can thus be seen as natural reservoirs from where they can theoretically spread into other environments and previously non-resistant microbial populations, including pathogens, in a process called horizontal gene transfer [45, 57, 193, 194]. The advantage that resistance genes confer to microorganisms exposed to anthropogenic antibiotic pressure is undisputed. However, the role of resistance genes in bacteria in environments not directly affected by application of antibiotics is less clear [19], and is not limited to antimicrobial defense. Bacteria can possess proteins that provide antibiotic resistance beyond their primary function [195]. For instance, Providencia stuartii possesses an enzyme involved in the acetylation of peptidoglycan, and the similarity of its substrate to certain aminoglycosides causes this enzyme to also provide antibiotic resistance [196].

Despite a growing body of knowledge about the distribution of antibiotic resistance genes in natural environments (i.e. those environments in which the human impact is limited), little is known about their expression, even though gene expression is a better proxy for functional activity than gene content [197]. We therefore hypothesized that a comprehensive metatranscriptome analysis could provide additional knowledge about resistance gene functioning and regulation. Due to the technical difficulties associated with processing of RNA samples, few metatranscriptome studies of microbial communities have been published [198-200]. However, recent advances, particularly in the field of next generation sequencing technologies, have resulted in increased availability of large metatranscriptome datasets $\left(>10^{6}\right.$ reads). In order to investigate the actual expression of resistance genes in natural environments, we evaluated both in-house generated as well as public metatranscriptomes. We investigated the expression levels and richness of genes conferring resistance to ten types of antibiotics, and furthermore assessed local production of these antibiotics by investigating the expression of relevant secondary metabolite gene clusters. 


\section{Results}

\section{Cumulative relative expression of antibiotic resistance genes}

Reads from metatranscriptome datasets from seven different ecological niches (human, mouse and pig intestinal microbiota, sea bacterioplankton, a marine sponge, forest soil and sub-seafloor sediment) were compared with genes from the resistance determinants database (RED-DB, www.fibim.unisi.it/REDDB) using megaBLAST. Per niche, a cumulative total was obtained for reads mapping to the different resistance genes, with the requirement that $\geq 3$ reads aligned per gene. In order to allow for comparison of gene expression in different datasets, we determined the relative abundance of resistance gene transcripts as a percentage of the total number of nonribosomal RNA reads. Expression of resistance genes was detected in all seven ecological niches (Figure 2.1), of which only the pig microbiota was knowingly exposed to antibiotics (i.e. a mixture of neomycin and procaine benzylpenicilline) approximately two to four weeks prior to taking the samples used for RNA extraction. Highest relative expression levels were observed in the sea bacterioplankton sample $(0.7 \%)$, whereas resistance gene transcripts comprised less than $0.06 \%$ of the reads in the other investigated niches. For human adults and pigs relative abundance of resistance gene transcripts was in the same order of magnitude $(0.009 \% \pm 0.014$ and $0.028 \% \pm 0.012$ [s.d.], respectively), whereas it was more than 15 -fold lower in mice $\left(4.4 \times 10^{-4} \% \pm 6.8 \times 10^{-4}\right)$ and the human infant $\left(5.7 \times 10^{-4} \%\right)$.

\section{Expression of genes conferring resistance to ten types of antibiotics}

The distribution of reads aligning to resistance genes that confer resistance to ten different types of antibiotics was analysed. Genes in the RED-DB are grouped according to resistance to the following types of antibiotics: aminoglycosides, $\beta$ lactams, chloramphenicol, glycopeptides, macrolides, oxazolidinones, quinolones, sulphonamides, tetracyclines and trimethoprim. Expression of genes involved in resistance to most types of antibiotics (6/10) was detected at least in one of the datasets analysed, with the exception of genes conferring resistance to quinolone, oxazolidinone, sulphonamide and trimethoprim antibiotics (Figure 2.2). In human gut microbiota $\beta$-lactam, aminoglycoside, macrolide and tetracycline resistance genes were expressed. In mice intestinal content samples, in which the cumulative relative expression of resistance genes was the lowest for all gut-associated niches examined here, primarily tetracycline resistance genes were found to be expressed. 


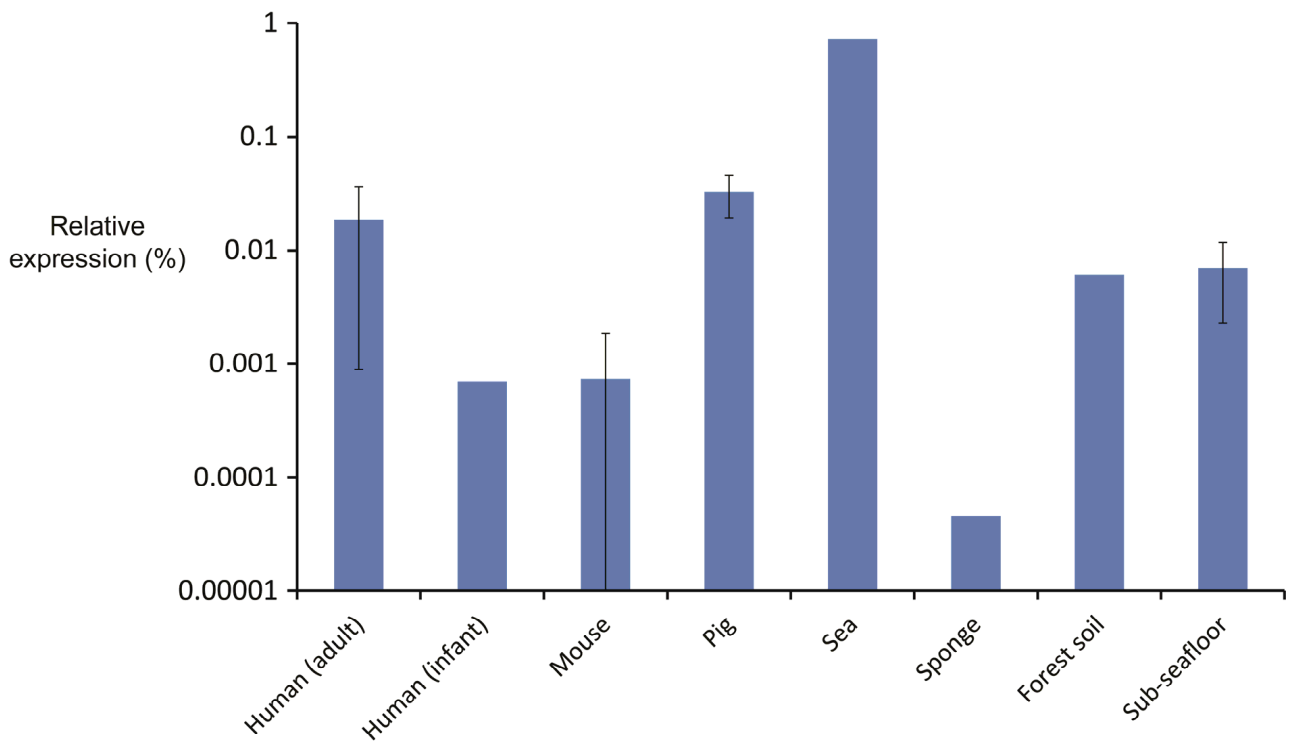

Figure 2.1 Relative cumulative expression of antibiotic resistance genes in different ecological niches. The relative expression is based on the total of number of reads that aligned to genes of the RED-DB, and calculated as a percentage of the non-ribosomal RNA reads. Error bars represent one standard deviation.

One-way ANOSIM showed that resistance profiles were not significantly different between different mammalian hosts when comparing the groups of human adults, mice and pigs (global $R=0.089 p=0.197$. A comparison of antibiotic resistance gene expression profiles of the microbiota of individual human adults, pigs and mice indicated there are substantial inter-individual differences within the groups (Figure 2.3). In the intestinal samples obtained from the human infant, only the expression of a tetracycline resistance gene was detected. The profiles of expressed resistance genes observed in forest soil and sub-seafloor sediment communities stood out because expression of chloramphenicol resistance genes made up $\geq 20 \%$ of the cumulative total whereas in the other ecological niches they were not detected. In sea bacterioplankton and the marine sponge the expression of two $\beta$-lactam resistance genes was detected, namely bla TEM-1 and bla ${ }_{\mathrm{TEM}-116 .}$. These genes share $97 \%$ nucleotide identity and therefore it is possible that in reality only a single $\beta$ lactam resistance gene is being expressed that is closely related to the two known genes. In sea bacterioplankton as much as $0.7 \%$ of the non-ribosomal transcripts aligned with the two bla TEM genes. 


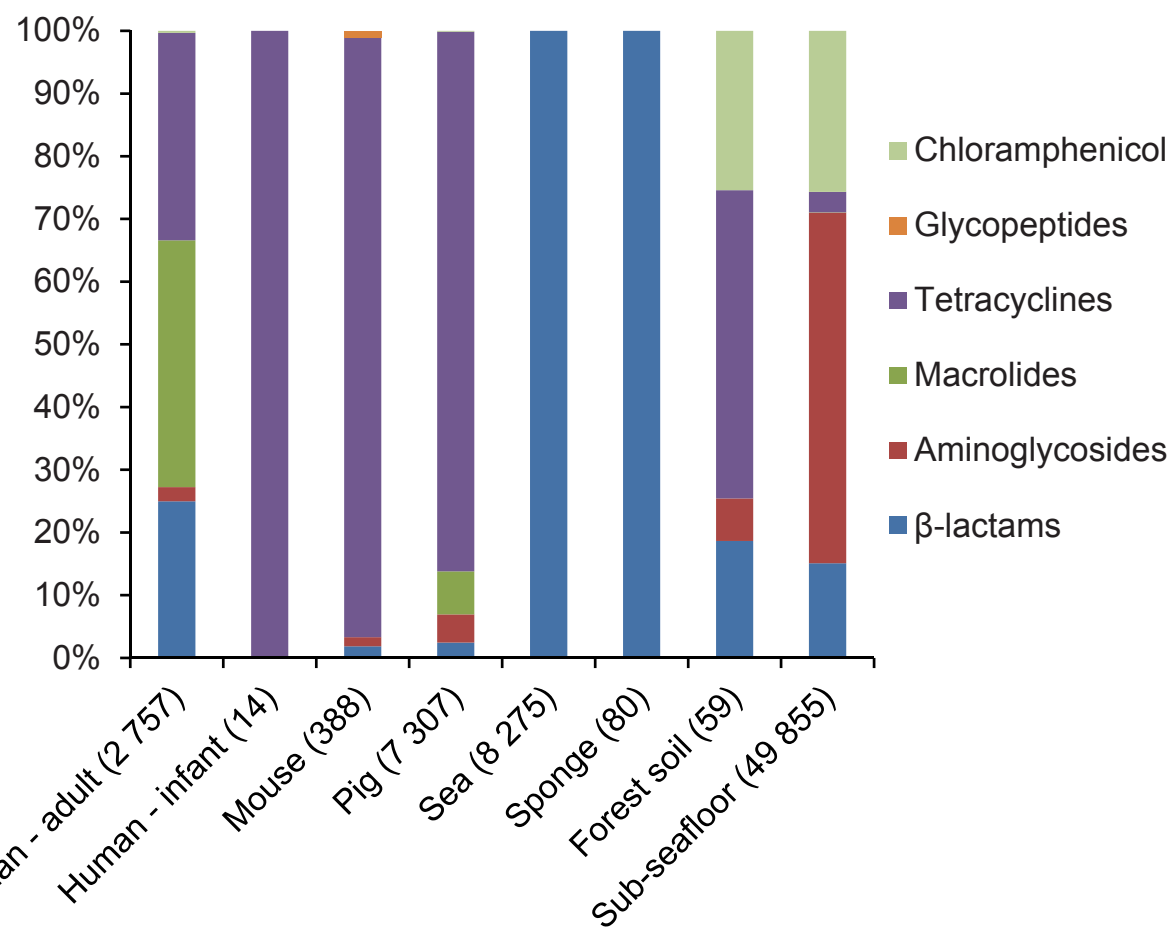

Figure 2.2 Resistance gene expression profiles for different ecological niches investigated for resistance to ten types of antibiotics. Genes conferring resistance to quinolone, oxazolidinone and trimethoprim antibiotics were not detected. The numbers in parentheses correspond to the total number of reads that aligned to resistance genes.

\section{Richness of expressed antibiotic resistance genes}

Read mapping was performed against single gene representatives of gene clusters defined by a $97 \%$ sequence identity threshold, in the following referred to as unique genes, and as a result, the number of detected genes can be taken as a measure for resistance gene richness. In human adult and pig microbiota the highest numbers of unique resistance genes were detected, namely 34 and 41 respectively. In the sea bacterioplankton and sponge metatranscriptomes, only two different resistance genes were detected. Detected resistance genes were classified according to which type of antibiotics they confer resistance (Figure 2.4, Table S2.2). It should be noted that the number of unique genes detected per niche is influenced by dataset size, sampling date, and the number of sampling sites from which data was analysed. Rarefaction curves in most cases (19/29) did not reach an asymptote, which suggests that resistance gene richness is underestimated to some extent (Figure S2.1). Regression analysis was performed to further investigate this feature for the 


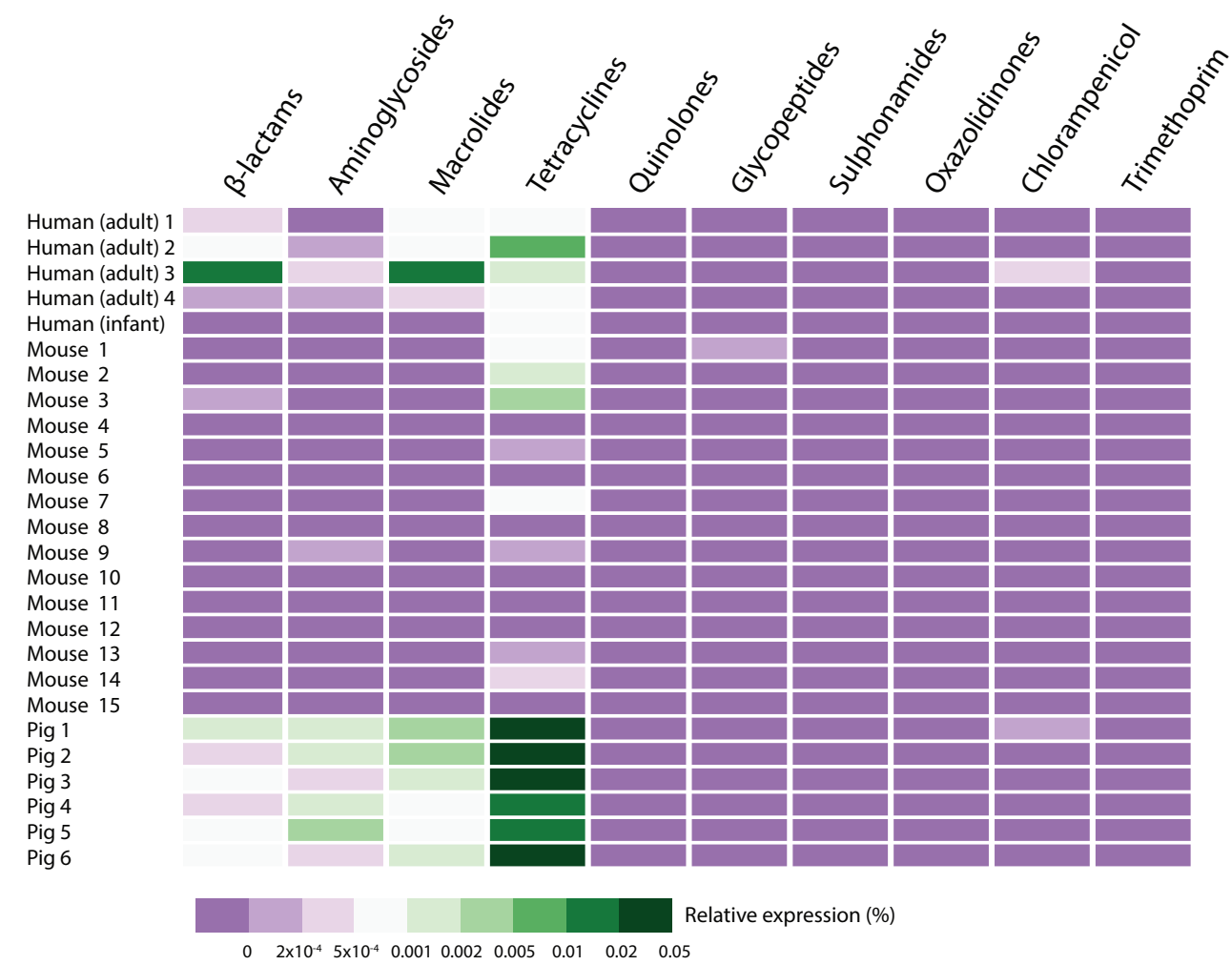

Figure 2.3 Heat map representing relative expression levels of resistance genes in the gut microbiota of individual humans, pigs and mice. Resistance genes are grouped based on resistance to ten types of antibiotics.

human and pig microbiota, as for these only the rarefaction curve of human adult individual 1 reached saturation (Table S2.1), and because the highest number of unique resistance genes was detected in these niches. Regression analysis equation two provided the best fit, of which the results indicate that in most cases $(8 / 10)$ the detected number of resistance genes is less than one gene different with respect to the expected number of resistance genes at infinite sample size. Therefore the datasets are expected to be sufficiently large to detect most of the resistance genes that were expressed.

\section{Expression of secondary metabolite biosynthesis (SMB) clusters}

We also investigated whether the expression of antibiotic resistance genes can be associated with local antibiotic biosynthesis. To this end, we used the antibiotics and secondary metabolite analysis shell (antiSMASH) pipeline, which focuses on the production of secondary metabolites, amongst which are also antibiotics [201]. Profile 


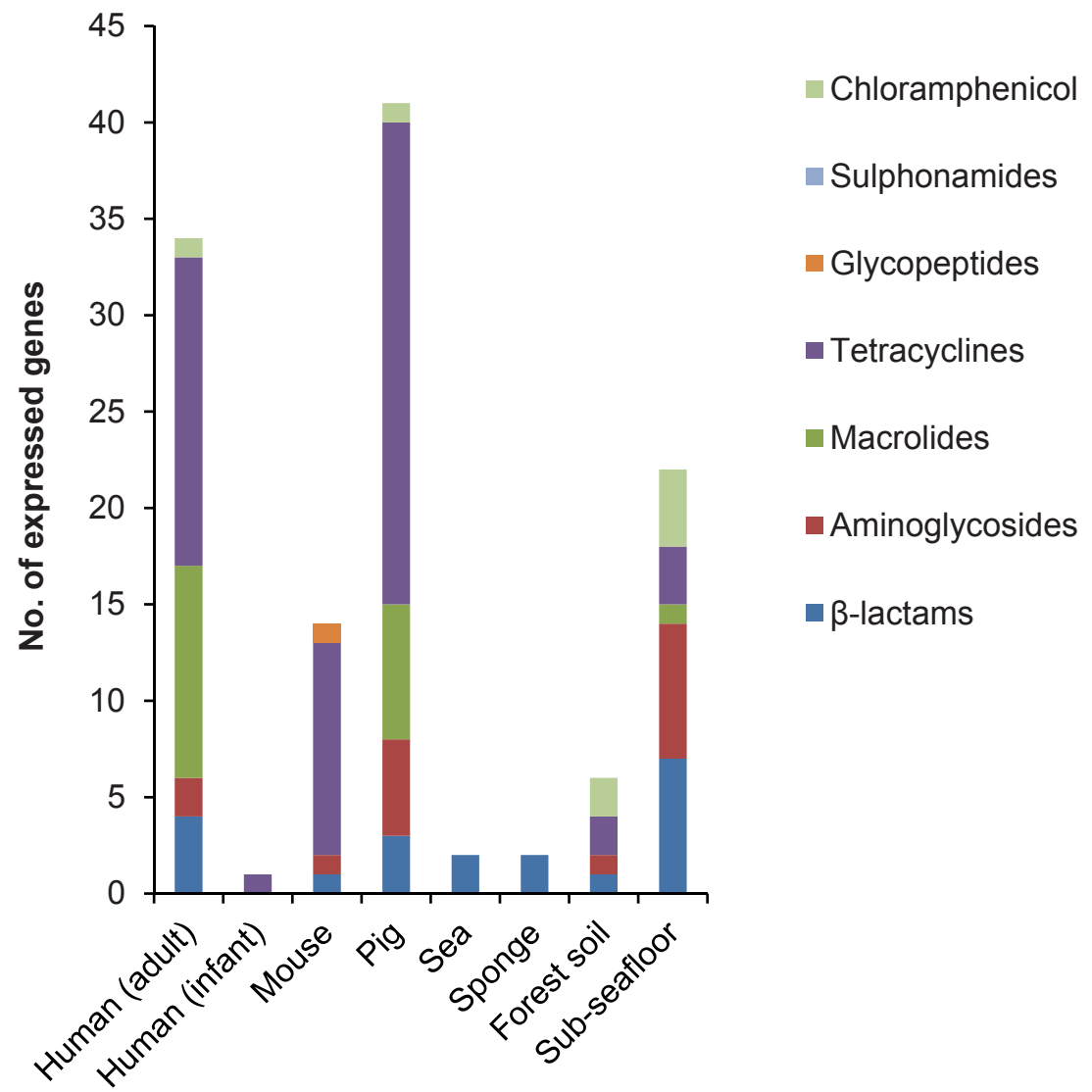

Figure 2.4 Richness of expressed resistance genes detected in the different ecological niches, based on the number of unique genes that were detected while using RED-DB entries clustered according to a $97 \%$ identity threshold.

hidden Markov Models (hMMs) from antiSMASH of domains of enzymes involved in the construction of the backbones of various types of antibiotics were used to investigate antibiotic biosynthesis. We investigated genes responsible for the biosynthesis of type I and II polyketides, non-ribosomal peptides and aminoglycosides/aminocyclitols (see Table S2.3 for the domains and the complete results), which can be involved in, respectively, the biosynthesis of macrolides, tetracyclines, $\beta$-lactams and aminoglycosides (Figure 2.5). In the gut microbiota of humans (adult and infant), pigs and mice, the marine sponge and sub-seafloor sediment, very little expression of SMB genes was detected. The lowest relative 
expression of SMB genes was found in human individual one, for whom only a single domain was detected by just five reads, suggesting that expression of SMB genes was very low. The expression of SMB genes in sea bacterioplankton and forest soil was at least an order of magnitude higher than in the other investigated environments. No associations between the expression of specific SMB clusters and resistance genes were uncovered, and their cumulative expression levels varied per ecological niche (Figure. S2.2).

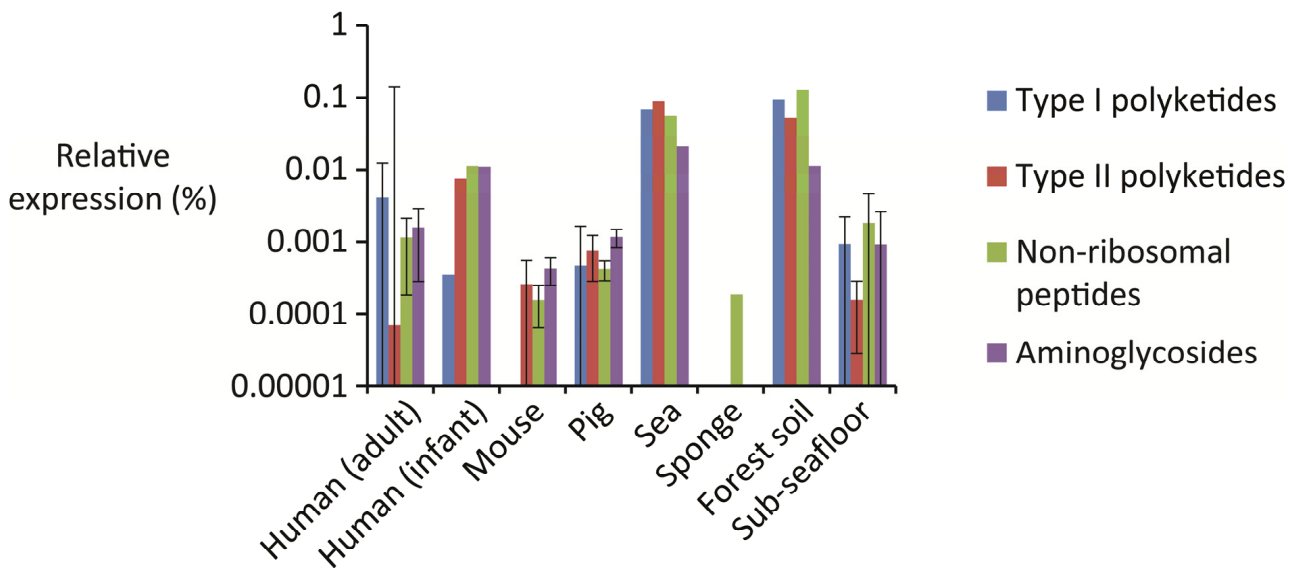

Figure 2.5 Cumulative relative expression of secondary metabolite biosynthesis domains involved in the production of type I polyketides, type II polyketides, non-ribosomal peptides and aminoglycosides. Error bars represent one standard deviation.

\section{Discussion}

Our explorative metatranscriptome analysis showed that antibiotic resistance genes are not only present, but also expressed in a broad variety of natural environments, exemplified here by samples derived from the intestinal tract of humans, mice, as well as from sea bacterioplankton, a marine sponge, forest soil and sub-seafloor sediment. Differences with respect to resistance gene expression profiles were observed between the ecological niches, and resistance genes richness was highest in human and pig microbiota. We find within-niche variability in resistance gene expression in gut microbiota of individual human adults, pigs and mice, which is consistent with metagenome-level studies wherein inter-individual variation in resistance potential was observed [104, 202]. It should be noted that the resistance profiles observed in this study are not necessarily representative for specific habitats since even samples from the same habitat but taken at different locations have been 
known to differ more than 100-fold in terms of relative expression levels of individual resistance genes [203]. On the other hand, microbial resistomes have been shown to cluster by habitat when soil, human and water resistomes were compared [105]. Expression of resistance genes against tetracyclines and $\beta$-lactams was most abundant in the gut microbiota of mammals and bacterial communities residing in aquatic environments, respectively. In the human gut microbiota specifically, resistance genes against $\beta$-lactams, macrolides and tetracyclines cumulatively comprised $>90 \%$ of the total expression. Resistance genes against these types of antibiotics were also most abundant in metagenome datasets of human gut microbiota [202]. We did not detect the expression of resistance genes against quinolone, oxazolidinone, sulphonamide and trimethoprim antibiotics. These antibiotics are all synthetic, and therefore these results might indicate that there has not yet been sufficient time for the corresponding resistance genes to spread into environmental reservoirs [108]. In the pig microbiota $\beta$-lactam and aminoglycoside resistance genes were detected, which might have resulted from these animals having received neomycin and procaine benzylpenicilline two to four weeks prior to sampling.

The expression of polyketide type I and II, non-ribosomal peptide, and aminoglycoside/aminocyclitol biosynthesis genes, which can potentially be involved in antibiotic biosynthesis, was detected in forest soil and sea bacterioplankton. This is consistent with previous reports [204-206]. In turn, in gut microbiota there was very little indication for potential antibiotic biosynthesis. Therefore, since antibiotic pressure seems to be absent in the sampled gut microbiota, it can be argued that antibiotic resistance gene expression in gut microbiota is either constitutive or controlled at least in part independently of the presence of antibiotics. Past studies already indicated that that expression of antibiotic resistance genes can be increased following mutations or movement of genetic elements [207]. As such, under antibiotic pressure, resistance genes that are constitutively expressed at low levels could become more active. For example, the expression of the ampC gene, which was detected in all human adults, has been shown to vary depending on a single mutation in the gene promoter [208].

There are two limitations to the undertaken approach to detect the expression of resistance genes that warrant consideration. The first limitation is that the number of characterized and deposited resistance genes in the RED-DB differs per ecological 
niche. As a consequence the number of expressed resistance genes can be underestimated if habitats have been studied less extensively. The second limitation is that we require the detection of 200 bp sequences instead of full-length resistance genes, and that as a result genes might be detected with other functional roles that share homology with resistance genes. Still, the cut-offs we used were conservative compared to those applied in previous studies [190, 209].

In human adult gut microbiota a large fraction of expressed resistance genes confer resistance to macrolide antibiotics. For this reason, and also since there is no indication of macrolide biosynthesis (in three out of four of the human adults), it can be suggested that the expression of macrolide resistance genes is either a result of prior anthropogenic antibiotic pressure as a result of which these genes are still constitutively expressed at a low level, or that the corresponding proteins serve other roles besides antibiotic resistance. A number of alternative physiological and metabolic functions have been proposed for two genes in M. tuberculosis that confer resistance to aminoglycosides, such as immune modulation and alleviation of cellular stress [210]. Nevertheless, the general consensus in literature is that the presence of antibiotic resistance genes in the gut microbiota is the result of anthropogenic antibiotic use, and there are numerous studies to support this claim [104, 211, 212]. In contrary to what was observed in humans and pigs in this study, in mice gut microbiota no macrolide resistance genes were found to be expressed, a result that could well be explained by lower exposure of mice to macrolide antibiotics, or antibiotics in general. This might also explain the overall lower levels and diversity of resistance gene expression detected in the gut microbiota of these animals. In the gut microbiota of the human infant expression of two tetracycline resistance genes was detected, as opposed to a variety of resistance genes in human adults. This could be because intestinal microbiota of babies generally have lower species diversity [211] and therefore species that contain resistance genes have not colonized the gut at levels that would allow detection of their transcripts. Remarkably, the human infant had not been exposed to antibiotics prior to the sampling and hence the observed naïve expression of tetracycline resistance genes indicates that antibiotics are not needed to trigger expression of cognate resistance genes. Indeed, one of the two transcribed tetracycline resistance genes shows high identity with the tetM gene which is known to be constitutively expressed but highly mobile as found in various conjugative transposons, such as $\operatorname{Tn} 916[212,213]$. 
Surprisingly, in sea bacterioplankton, the expression of AR genes was more than one order of magnitude higher than in any other ecological niche. It was comprised of two highly similar $\beta$-lactamase genes, namely blaTEM-1 and blaTEM-116. The same two genes were also found to be expressed in a metatranscriptome dataset from the marine sponge Crambe crambe. The bla $\mathrm{TEM}_{\mathrm{TE}} \mathrm{g}$ gene is commonly present in cloning vectors, and therefore lab contamination can be suspected; however, this possibility is disputed by the fact that the gene is detected in two separate marine metatranscriptome datasets that have been independently generated. The functional role of a $\beta$-lactamase in a marine environment is yet unknown. A substrate for this enzyme might be periplasmic or cell-wall bound, as excreted $\beta$-lactams could quickly be diluted to negligible concentrations in the open sea. On the other hand, the $\beta$-lactamase could also have an extra-cellular role if the bacteria occasionally reside in places with a high cell density (micro niches), such as corals or sponges. Roles of these resistance genes in (the disruption of) cell signalling and antimicrobial activity can be hypothesized [19]. Another possibility is that these $\beta$-lactamases have no role in the aquatic environment but that they appeared through resistance gene dissemination, such as via human-derived sewage or animal faeces [100].

Acquired resistance to antibiotics is often associated with a fitness cost, which translates itself into a reduced bacterial growth rate. It has therefore been proposed that in an antibiotic-naïve environment in the long term, resistant strains will be outcompeted by susceptible strains [214, 215]. Still, Andersson and Hughes (2010) argued that reversal to a susceptible state proceeds so slowly, if at all, that the fitness cost has no practical importance. Moreover, it cannot be excluded that resistance genes co-localize, i.e. they are genetically linked, or that compensatory mutations have occurred that abolish fitness costs. It has been shown that selection for resistant bacteria occurs at antibiotic concentrations far below the minimal inhibitory concentration [216]. This suggests that resistance can be maintained in bacterial populations even if antibiotics are produced locally at a low concentration, or as a result of anthropogenic intervention.

In summary, a broad diversity of resistance genes was found expressed under natural conditions in the gut microbiota of mammals, as well as in aquatic and terrestrial bacterial communities, with niche-specific differences in relative expression levels and diversity of transcripts. Although the expression of antibiotic resistance 
genes could not be associated with expression of relevant secondary metabolite biosynthesis clusters, we do see a rich functional reservoir of resistance genes that could be accessible by human pathogens.

\section{Materials and methods}

\section{Metatranscriptome data}

In addition to in-house generated metatranscriptome datasets, literature was searched for available metatranscriptome datasets that contain at least $1 \times 10^{6}$ reads (see Table S2.4 for all datasets included in this study and their accession numbers). The analysed metatranscriptome data were generated from the intestinal microbiota of four human adults, one human infant, six pigs and 15 mice, as well as from the orange-red encrusting sponge (Crambe crambe), forest soil [217], sub-seafloor sediment [218] and sea bacterioplankton [219]. Except for the pig microbiota, none of the environments that were sampled did experience anthropogenic antibiotic pressure. The pig microbiota metatranscriptome data were included because pigs regularly receive antibiotics during intensive farming, and as such expression of resistance genes in this niche is of particular interest [220]. The human adult metatranscriptome data were obtained from ileostoma effluent samples of four human adults [221], as described by Leimena et al. [222]. The metatranscriptome data of the human infant was generated from faecal samples collected from a Finnish baby girl at three time points in the first six months of life (at the ages of 131 [sample 2B], 165 [samples 2D_1 and 2D_2] and 171 [sample 2E] days). Multiple time points were included (data from multiple time points was studied as a single entity) because the gut microbiota of human babies is very dynamic [211, 223]. Approximately $0.5 \mathrm{~g}$ of each faecal sample was processed with the RNAlater protocol [224] and stored at $70{ }^{\circ} \mathrm{C}$ prior to RNA isolation. RNA isolation was done with a protocol based on mechanical disruption [224], after which the quality and yield of the RNA was assessed with the NanoDrop 1000 Spectrophotometer (Thermo Scientific). Sequencing was performed on a Illumina HiSeq 2500 instrument. An additional presequencing rRNA removal step with the Rib-Zero kit (Epicentre) was included for sample 2D_2. The pig metatranscriptome data were those generated from proximal colon samples of pig 2,pig 4, pig 5, pig 6, pig 8 and pig 10 from a study by Haenen et al. [225], and in this study these pigs are addressed as pigs 1 to 6 , respectively. It is important to note that the pigs received Neopen (MSD Animal Health) intramuscularly 
prior to sampling. The mice metatranscriptome data were generated from cecum samples of 15 mice from a study by Hugenholtz et al. [226], and both pig and mice samples were processed and sequenced as described in this study. The sponge (Crambe crambe) metatranscriptome data were obtained from a sample that was collected as described before [227]. A one $\mathrm{cm}^{3}$ piece of sponge tissue was fixed in ten volumes of RNALater. Subsequently, RNA was extracted with a modified guanidium thiocyanate phenol-chloroform extraction method [228] where $100 \mathrm{mg}$ of tissue sample was homogenized in Solution D with glass beads in a Precellys 24 tissue homogenizer (Bertin Technologies) prior to extraction. The total RNA was treated with the Dynabeads $₫$ mRNA Purification Kit (Life Technologies) to remove sponge mRNA. One $\mu \mathrm{g}$ of the resulting RNA sample was treated with both the Epibio Ribo-Zero rRNA (Illumina) removal kit for Human/mouse/rat and the Epibio Ribo-Zero rRNA (Illumina) removal kit for meta-bacteria in order remove sponge rRNA and bacterial rRNA, respectively. Ten ng of resulting enriched bacterial mRNA was reverse transcribed and amplified with the Ovation RNASeq system (Nugen). Sequencing was performed on a Illumina HiSeq 1000 instrument at Macrogen. In order to perform a thorough analysis for the sub-seafloor sediment, available metatranscriptome data from all six depths ( $\geq 5$ metres below the seafloor) was analysed.

\section{Calculation of the fraction of non-ribosomal RNA reads}

In order to allow for comparison of relative transcript abundance across different datasets, we determined the number of non-ribosomal RNA reads for each metatranscriptome, applying the SortMeRNA pipeline [229], and using the included rRNA database and default filtering settings.

\section{Analysis of resistance gene expression}

To determine the expression of antibiotic resistance genes, the metatranscriptome reads were compared with the genes present in the resistance determinants database (RED-DB, www.fibim.unisi.it/REDDB). Genes in the RED-DB are grouped according to the type of antibiotic they confer resistance to, which makes the database suitable for our purpose. Relying on the FASTA headers of resistance genes (i.e. if FASTA headers matched the string "fusion"), fusion proteins of resistance genes together with sequences unrelated to antibiotic resistance were removed from the database. Subsequently, the redundancy of the RED-DB was 
reduced by clustering of entries with CD-HIT-EST [230] according to a $\geq 97 \%$ nucleotide identity threshold, reducing it in size to 4,537 genes. Thereafter, megaBLAST [231] was used to align the metatranscriptome reads against the nonredundant RED-DB with a nucleotide identity of $\geq 90 \%$ and an alignment length of $\geq 60$ bp as alignment thresholds. For a gene to be considered expressed at least three reads were required to align with a cumulative covered gene length of $\geq 200 \mathrm{bp}$. These cut-offs are more stringent than those used in previous studies [190, 209]. When these cut-offs were made less stringent, no notable increase in the expression of antibiotic resistance genes was observed, indicating the robustness of this approach (data not shown). The hit list of resistance genes was manually inspected. Housekeeping genes that are targets of antibiotics and can become resistance genes through mutations (e.g. DNA gyrase and RNA polymerase subunit B) were removed, as well as genes that cannot always be directly associated with a resistance phenotype (e.g. ribF [232]] and sanA [233]]). One-way analysis of similarities [234] (ANOSIM, Bray-Curtis distance measure, 999 permutations) was performed with the PRIMER 6 software package (Clarke, K.R., Gorley, R.N., 2006. PRIMER v6: User Manual/Tutorial. PRIMER-E, Plymouth) to test for significant differences between the expression profiles in human adults, mice and pigs, using the cumulative relative expression levels of resistance genes against each of the 10 types of antibiotics. Rarefaction curves were prepared using the QIIME script multiple_rarefactions.py. The average number of resistance genes that were detected by $>3$ reads in the rarefied OTU tables was calculated after 1,000 iterations of subsampling. Rarefaction curves were plotted and regression analysis was performed using two different equations [235].

$y=a\left(1-e^{-b x}\right)$

$y=a\left(1-e^{-b x^{\wedge} c}\right)$

where $\mathrm{x}$ is the sample size (number of reads sampled), $\mathrm{y}$ the observed number of resistance genes and a the number of resistance genes to be expected with infinite sample size.

\section{Expression of secondary metabolite biosynthesis cluster genes}

Various secondary metabolite biosynthesis (SMB) gene clusters encode proteins needed to produce antibiotics, and therefore their expression can give information 
about the potential presence of naturally produced antibiotics in a given ecological niche. For the construction of the antibiotics and secondary metabolite analysis shell (antiSMASH) [201], a list of core domains was compiled, which are exclusively present in genes in specific types of SMB clusters. Profile Hidden Markov Models (pHMMs) are available for these domains. In order to detect the expression of SMB clusters, metatranscriptome reads were translated to protein sequences in all six open reading frames. Subsequently, the HMMer3 tool (http://hmmer.janelia.org/) was used to detect the core domains specific to the different SMB clusters. The SMB clusters for which expression was investigated included those involved in synthesis of non-ribosomal peptides, polyketides type I and II and aminoglycosides/aminocyclitols. Thresholds for domain detection were the trusted cut-offs supplied with the pHMMs, and when those were not available an E-value of $1.0 \times 10^{-7}$ was used. Core domains were required to be detected by at least three reads in order for them to be considered expressed.

\section{Acknowledgements}

This work was supported by the European Union through the EvoTAR project (Grant agreement no. 282004). The sequencing of metatranscriptomes of human, mouse and pig origin was partially funded through TI Food \& Nutrition, the Netherlands Consortium for Systems Biology (NCSB) which is part of the Netherlands Genomics Initiative / Netherlands Organisation for Scientific Research, as well as the European Union through the Interplay project (Grant agreement no. 227549) and Cargill's R\&D Center Europe (Vilvoorde, Belgium). Cargill had no role in the study design, data collection and analysis, decision to publish, or preparation of the manuscript. This work was also supported in part by grants 141140 and 1272870 from the Academy of Finland to WMdV. We thank MetaExplore (EU Grant agreement no. 222625) for making available the sponge metatranscriptome data in advance of publication. 


\section{Supplementary information}

\section{Supplementary Tables and Figures}

Tables S2.2, S2.3 and S2.4 are available on the website of Scientific Reports; doi: 10.1038/srep11981.

Table S2.1 Regression analysis was done to fit the rarefaction curves using 2 equations: (f1) y = a (1 - e-bx) and (f2) y =a $\left(1-\mathrm{e}-\mathrm{bx}^{\wedge} \mathrm{c}\right)$. Constant a indicates the number of resistance genes to be expected with infinite sample size. In the case of human 1 no good fit was obtained for equation $\mathrm{f} 1$.

\begin{tabular}{|c|c|c|c|c|c|c|c|c|}
\hline Sample & $\begin{array}{l}\text { No. of resistance } \\
\text { genes detected }\end{array}$ & a (f1) & b (f1) & $r^{2}$ & $a(f 2)$ & b (f2) & c (f2) & $r^{2}$ \\
\hline Human 1 & 5 & n.a. & n.a. & n.a. & 5.245 & $2.05^{*} 10^{-15}$ & 2.485 & 0.9997 \\
\hline Human 2 & 20 & 20.34 & $6.76^{*} 10^{-07}$ & 0.9962 & 20.34 & $6.49 * 10^{-07}$ & 1.003 & 0.9962 \\
\hline Human 3 & 19 & 18.76 & $1.14^{*} 10^{-06}$ & 0.9064 & 24.19 & $7.25^{\star} 10^{-05}$ & 0.677 & 0.9974 \\
\hline Human 4 & 20 & 20.93 & $1.89 * 10^{-07}$ & 0.9956 & 20.67 & $1.49 * 10^{-06}$ & 0.857 & 0.9986 \\
\hline Pig 1 & 35 & 33.86 & $1.27 * 10^{-06}$ & 0.9621 & 35.971 & $9.73^{*} 10^{-05}$ & 0.676 & 0.9996 \\
\hline Pig 2 & 25 & 24.77 & $1.78^{*} 10^{-06}$ & 0.9973 & 24.456 & $6.73^{*} 10^{-07}$ & 1.075 & 0.9983 \\
\hline Pig 3 & 25 & 26.05 & $1.46^{*} 10^{-06}$ & 0.9862 & 32.344 & $3.24 * 10^{-05}$ & 0.742 & 0.9972 \\
\hline Pig 4 & 25 & 30.02 & $8.36^{*} 10^{-07}$ & 0.9957 & 26.251 & $8.34^{*} 10^{-08}$ & 1.185 & 0.9987 \\
\hline Pig 5 & 31 & 30.10 & $1.15^{*} 10^{-06}$ & 0.9893 & 31.884 & $1.25^{*} 10^{-05}$ & 0.818 & 0.9967 \\
\hline Pig 6 & 27 & 25.15 & $1.42 * 10^{-06}$ & 0.9763 & 27.235 & $2.05^{*} 10^{-05}$ & 0.791 & 0.9857 \\
\hline
\end{tabular}

A)

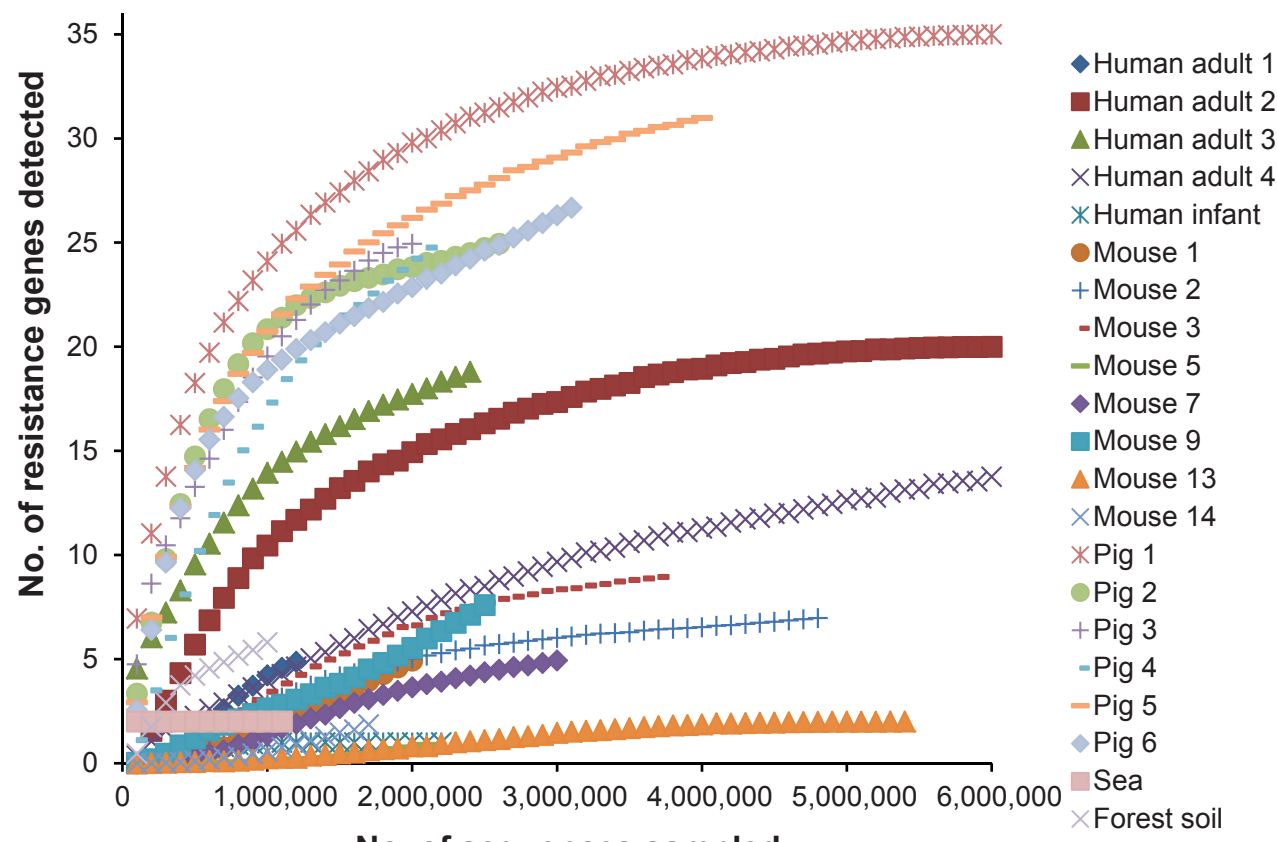

No. of sequences sampled 
B)

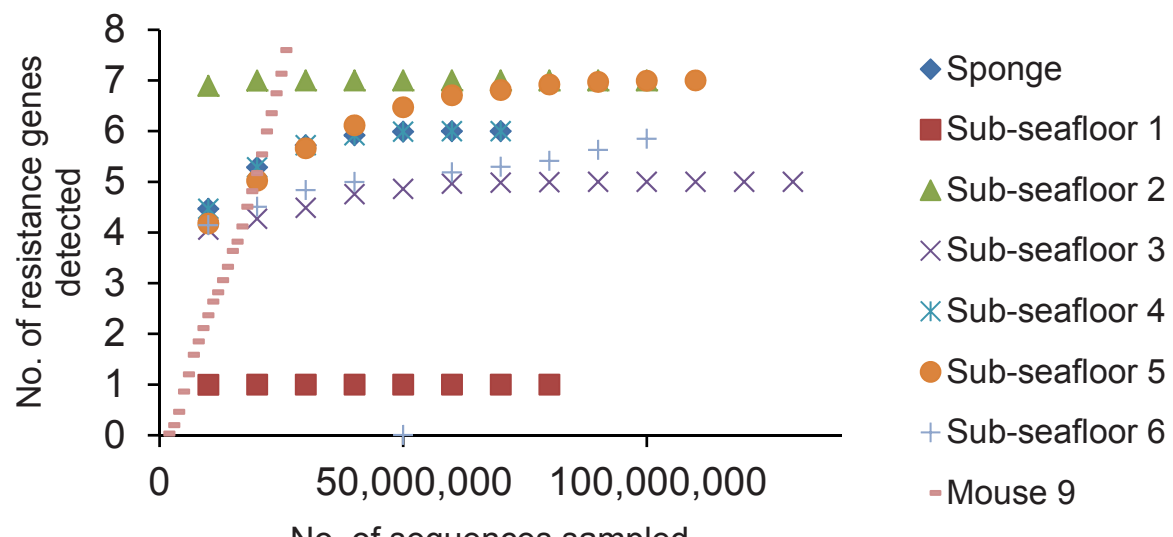

Figure S2.1 Rarefaction curves for all investigated ecological niches: (A) for datasets with $<20,000,000$ non-ribosomal RNA reads (B) for datasets with $>20,000,000$ non-ribosomal RNA reads.

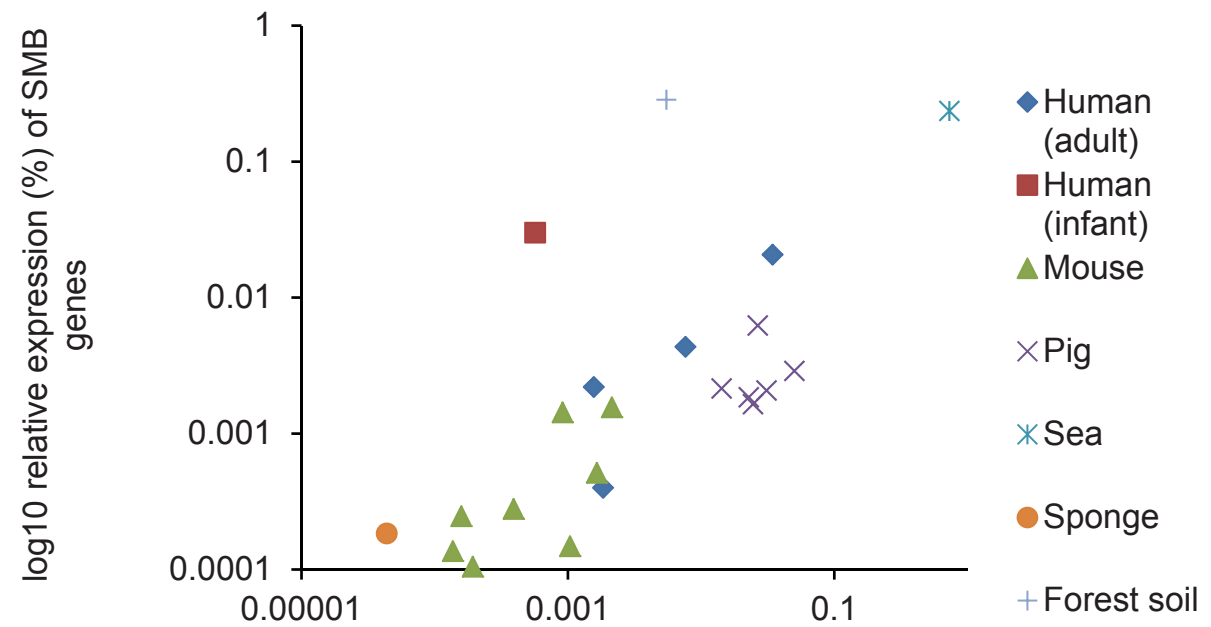

log10 relative expression (\%) of AR genes

Figure S2.2 Relative cumulative expression of antibiotic resistance genes plotted against the cumulative relative expression of secondary metabolite biosynthesis domains involved in the production of type I polyketides, type II polyketides, non-ribosomal peptides and aminoglycosides. 



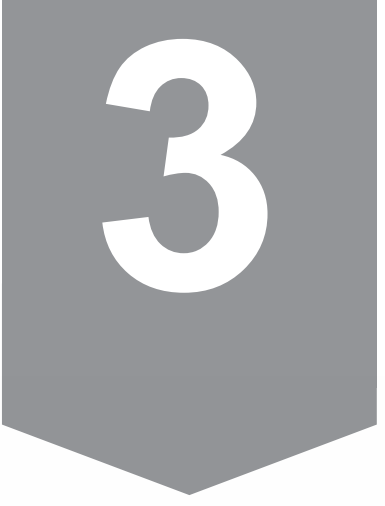

High throughput recovery of previously uncultured bacterial genera from three Mediterranean sponges 


\section{Abstract}

Sponges often harbour a dense and diverse microbial community. Presently, a large discrepancy exists between the cultivable bacterial fraction from sponges and the community in its natural environment. Here, we aimed to acquire additional insights into cultivability of (previously uncultured) bacteria from three sponge species, namely Aplysina aerophoba, Corticium candelabrum and Petrosia ficiformis, by studying bacterial growth on five media in the form of 60 communities scraped from plates without antibiotics, as well as in the form of individual isolates that were grown on these media supplemented with antibiotics. We applied (double-)barcoded $16 \mathrm{~S}$ rRNA gene amplicon sequencing for species identification. We show that previously uncultured bacteria can be cultivated using conventional plating and that application of antibiotics in the media can serve to capture a greater bacterial diversity. Moreover, we present criteria to address an important caveat of the plate scraping method whereby bacteria may be detected that did not actually grow. Fourteen out of 27 cultivated novel taxa ( $<95 \%$ identity of the 16S rRNA gene amplicon to reported species) belong to Actinobacteria, which indicates the presence of a large untapped reservoir of bioactive compounds. Three Flavobacteriaceae spp. were isolated that potentially constitute two new genera and one new species. 


\section{Introduction}

Sponges arose 600-700 million years ago and have been consistent members of benthic communities ever since [116]. Their diet consists of organic debris and plankton collected from the surrounding water through filter feeding. Even though sponges actively feed on microorganisms, they often simultaneously harbour dense and diverse microbial communities that co-exist with the host in a mutualistic relationship, and together are referred to as the sponge holobiont [117].

Sponges are generally distinguished according to the bacterial cell density in their tissues where they either have a bacterial concentration within the range of surrounding sea water (low-microbial-abundance sponges), or the bacterial concentration is increased by 2-4 orders of magnitude (high-microbial-abundance sponges) [124]. A comprehensive survey of publicly available 16S rRNA gene sequences of sponge-associated bacteria showed that, overall, (in descending order of relative abundance) Proteobacteria, Actinobacteria, Bacteroidetes, Chloroflexi and Firmicutes are the dominant bacterial phyla [123]. However, there is great variation between sponge-associated microbial communities, and remarkably, bacterial communities seem to be largely host species-specific, with only few bacterial lineages shared between sponge species [126-128]. Furthermore, host speciesspecific microbiota were found to be relatively stable in time, and [236] over wide bathymetric and geographic gradients [237]. Sponge-specific bacteria are of particular interest in the study of the complex host-symbiont relationship since they may represent the oldest animal-microbe symbioses on Earth [238].

However, little is known regarding the metabolic functions and possible hostmicrobe interactions of sponge-associated bacteria. Functional roles that have been ascribed to sponge microbes are carbon fixation [239], nitrogen cycling [125, 240] and host defense [241-243]. Host defense is suggested in most cases to be the result of microbe-derived bioactive compounds, and in the last decade their potential pharmaceutical application has sparked numerous studies [134, 135]. The classical non-ribosomal polyketide synthethase (NRPS) and polyketide synthase (PKS) pathways responsible for secondary metabolite production in soil microbes have also been found in sponge bacteria, including novel variations that appear to be restricted to symbiotic bacteria [244-246]. Still, bioactive compounds from sponge-associated 
bacteria are rather unexplored, a phenomenon that can be attributed to spongeassociated bacteria being difficult to culture.

Cultivation of sponge-associated bacteria is desirable in order to obtain a comprehensive understanding of their biology and ecological role [93]. However, there is still a large discrepancy between the cultivable bacterial fraction from sponges and the community in its natural environment [129, 130]. To address this gap between presence in the natural environment and cultivation, we extensively studied the cultivability of sponge-associated bacteria from three Mediterranean highmicrobial-abundance sponges, namely Aplysina aerophoba, Petrosia ficiformis and Corticium candelabrum, by collecting total colony material obtained under different cultivation conditions, and performing community analysis via sequencing. In addition, colonies were picked from media supplemented with different antibiotics in an effort to select for different bacterial species.

\section{Materials and methods}

\section{Sample collection}

Tissue samples were collected from one individual per sponge species. Samples from $P$. ficiformis (Pf1) and C. candelabrum (CC1) were collected on June $5^{\text {th }} 2008$

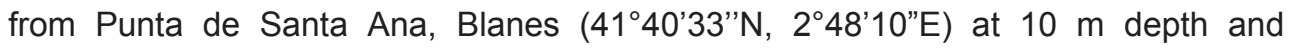
identified by M.J. Uriz. A. aerophoba (AA3) samples were collected on January $15^{\text {th }}$ 2012 from Cala Montgo (42 $06^{\prime} 52.20^{\prime \prime} \mathrm{N}, 03^{\circ} 10^{\prime} 06.52$ 'E) at $12 \mathrm{~m}$ depth and identified by D. Sipkema. Specimens were brought to the surface in plastic ziplock bags. The sponge samples were rinsed three times with sterile artificial sea water (ASW, $33 \mathrm{~g} / \mathrm{l}$ synthetic sea salt [Instant Ocean Reef Crystals, Aquarium Systems, Sarrebourg, France]) and were cut into pieces of $\sim 0.1 \mathrm{~cm}^{3}$. Half of the pieces was stored in $100 \%$ ethanol, while the other half was homogenized with mortar and pestle, and two tissue volumes of sterile artificial sea water were added to obtain a homogeneous cell suspension. This suspension was aliquoted in $1.2 \mathrm{ml}$ fractions, and each fraction was mixed with $0.6 \mathrm{ml} 50 \%$ sterile glycerol in ASW before storage at $-80^{\circ} \mathrm{C}$.

\section{Cultivation conditions}

Isolation of bacteria on media with antibiotics

Material from the original glycerol stocks was 10-fold diluted in ASW and subsequently $75 \mu \mathrm{l}$ was used to inoculate one plate of each combination of five media and 13 combinations of antibiotics. Fifty $\mu$ l was in contact with agar whereas 
the other $25 \mu \mathrm{l}$ was inoculated on a $0.2 \mu \mathrm{m}$ GTTP isopore membrane filter (Millipore) that was placed on top of the agar. The following five media were used: (I) $37.40 \mathrm{~g} / \mathrm{l}$ marine broth 2216 (Difco) in Milli-Q; (II) $3.74 \mathrm{~g} / \mathrm{l}$ marine broth 2216 (Difco) in Milli-Q ; (III) $22 \mathrm{~g} / \mathrm{l} \mathrm{BBL}$ Mueller Hinton broth (BD) in ASW; (IV) $2.2 \mathrm{~g} / \mathrm{l} \mathrm{BBL}$ Mueller Hinton broth (BD) in ASW; (V) $1 \mathrm{~g} / \mathrm{l}$ porcine stomach mucin (Sigma) in ASW. All media contained $15 \mathrm{~g} / \mathrm{l}$ noble agar (Sigma-Aldrich). Media III to $\mathrm{V}$ were supplemented with 1 $\mathrm{ml} / /$ trace metal solution [247], $1 \mathrm{ml} / \mathrm{l}$ phosphate solution [247] and $1 \mathrm{ml} / \mathrm{l}$ vitamin solution (BME vitamins, Sigma), with the $\mathrm{pH}$ adjusted to 7.7 before autoclaving. Solutions of phosphate, vitamins and antibiotics were filter-sterilized $(0.2 \mu \mathrm{m})$ and added after autoclaving in order to prevent precipitation or inactivation. The following 13 (combinations of) antibiotics were added: (I) $15 \mu \mathrm{g} / \mathrm{ml}$ polymyxin B and $15 \mu \mathrm{g} / \mathrm{ml}$ linezolid; (II) $15 \mu \mathrm{g} / \mathrm{ml}$ polymyxin B and $15 \mu \mathrm{g} / \mathrm{ml}$ daptomycin; (III) $15 \mu \mathrm{g} / \mathrm{ml}$ polymyxin $\mathrm{B}$ and $50 \mu \mathrm{g} / \mathrm{ml}$ vancomycin; (IV) $15 \mu \mathrm{g} / \mathrm{ml}$ polymyxin B and $50 \mu \mathrm{g} / \mathrm{ml}$ penicillin; (V) 15 $\mu \mathrm{g} / \mathrm{ml}$ erythromycin; (VI) $15 \mu \mathrm{g} / \mathrm{ml}$ ciprofloxacin; (VII) $100 \mu \mathrm{g} / \mathrm{ml}$ cefotaxime; (VIII) 50 $\mu \mathrm{g} / \mathrm{ml}$ tetracycline; (IX) $50 \mu \mathrm{g} / \mathrm{ml}$ chloramphenicol; (X) $95 \mu \mathrm{g} / \mathrm{ml}$ sulfamethoxazole and $5 \mu \mathrm{g} / \mathrm{ml}$ trimethoprim; (XI) $15 \mu \mathrm{g} / \mathrm{ml}$ lincomycin; (XII) $100 \mu \mathrm{g} / \mathrm{ml}$ kanamycin; (XIII) 7.5 $\mu \mathrm{g} / \mathrm{ml}$ rifampicin. Plates were incubated in the dark at $20{ }^{\circ} \mathrm{C}$ for three months. Colonies were picked twice per week in the first month and subsequently once per week. At most three colonies of the same morphology were picked per plate. Colonies were transferred to a fresh plate and colony material from these plates was used for preparation of glycerol stocks. Colonies appearing on filters were immediately stored in glycerol. Glycerol stocks were prepared by transferring colony material to 96 -well plates containing $25 \%$ glycerol in ASW, and stored at $-80{ }^{\circ} \mathrm{C}$. In addition, colony material was transferred to 96-well plates containing nuclease-free water (Promega) to be used as direct template for 16S rRNA gene PCR.

\section{Bacterial growth on media without antibiotics}

Material from the original glycerol stocks of $A$. aerophoba, $P$. ficiformis and $C$. candelabrum was respectively 100-fold, 10-fold and 2-fold diluted in ASW based on prior CFU counts, and subsequently plated on six plates of each of the five media described previously. Seventy-five $\mu$ l of diluted material was inoculated on each plate (50 $\mu \mathrm{l}$ in contact with agar and $25 \mu \mathrm{l}$ on a filter membrane on top of the agar). The plates were stored at $20{ }^{\circ} \mathrm{C}$ in the dark. Colony material from each combination of media and sponges was harvested 15 days ( 3 plates) and 30 days (the remaining 3 
plates) post-incubation by plate washing with the help of an L-shaped spreader. Colony material on top of agar and filter membranes was collected separately in $3 \mathrm{ml}$ phosphate buffered saline (PBS, $137 \mathrm{mM} \mathrm{NaCl}, 2.7 \mathrm{mM} \mathrm{KCl}, 10 \mathrm{mM} \mathrm{Na}{ }_{2} \mathrm{HPO}_{4}$, $\mathrm{KH}_{2} \mathrm{PO}_{4}$ ) each. $2.5 \mathrm{ml}$ was stored for gDNA isolation at $-20{ }^{\circ} \mathrm{C}$. The rest was mixed with $0.5 \mathrm{ml} 50 \%$ glycerol (in ASW) and stored at $-80{ }^{\circ} \mathrm{C}$ for cultivation.

\section{DNA extraction and 16S rRNA gene amplicon sequencing}

\section{DNA extraction}

The DNeasy Blood \& Tissue Kit (Qiagen) was used to extract total DNA from $0.4 \mathrm{~cm}^{3}$ of sponge tissue as well as from colony material stored in PBS (the fractions that were washed from the plates without antibiotics), according to the manufacturer's protocol for animal tissue. The DNA concentration was determined by NanoDrop (Thermo Scientific). DNA samples were stored at $-20{ }^{\circ} \mathrm{C}$ in AE buffer.

\section{Barcoded 16S rRNA gene amplicon 454-pyrosequencing}

Barcoded 16S rRNA gene amplicon 454-pyrosequencing was done (1) to analyse bacterial communities present in the sponge samples, (2) to identify bacterial colonies that were picked from media containing antibiotics, and (3) to analyse bacterial communities retrieved by scraping from media without antibiotics. PCR was performed to amplify an approximately $311 \mathrm{bp}$ fragment comprising the V1 and V2 regions of the 16S rRNA gene. For this purpose the extracted DNA of the samples was diluted to $10-20 \mathrm{ng} / \mu \mathrm{l}$. In case of identification of bacterial colonies cell material in nuclease-free water served as template. The composite forward primer consisted of titanium sequencing adaptor A, a barcode [248] and degenerate primer 27F-DegS [249] (Table S3.1). Reverse priming was done by an equimolar mixture of primers 338R-I and 338R-II [250], each attached to titanium sequencing adaptor B. For the identification of individually picked colonies a barcode was also included in the reverse primer (for each colony a unique combination of barcodes was used). PCR conditions and library preparation are described in the Supplementary Materials and Methods. Five libraries were sent for sequencing that contained samples particular to this study (Table S3.1). 
Demultiplexing, quality-filtering, denoising and taxonomy assignment of 454pyrosequencing data

454-pyrosequencing reads were processed with QIIME [251]. Firstly, libraries were demultiplexed according to the forward primer barcodes. Reads were retained if the average Phred quality score was $>25$, no homopolymer stretches $>6$ nucleotides were present, the read length was $\geq 200 \mathrm{bp}$, and no mismatches were present in the 5 '-end primers. For 454-pyrosequencing reads that were double-barcoded (i.e. those generated from picked colonies), an additional demultiplexing step based on the reverse barcode was performed. Subsequently, reads were denoised by Acacia [252] using default settings. Next, open reference operational taxonomic unit (OTU) picking and taxonomic assignment was performed by uclust [253] based on a 97\% identity threshold and the representative sequences of the $97 \%$ identity clustered SILVA 111 database as a reference. The representative sequence from each OTU was picked, which is the centroid that was used for OTU picking. Potentially chimeric OTUs were identified using ChimeraSlayer and the downloadable Greengenes core_set_aligned.fasta.imputed, and were excluded from downstream analysis. Finally, OTU tables were generated that tabulate which OTUs, orders or phyla were detected in each sample, and by how many reads.

\section{Analyses and visualisation of bacterial diversity}

\section{Diversity and clustering analyses of bacterial communities}

For sponge bacterial communities, rarefaction curves were calculated with QIIME scripts. Hierarchical clustering was performed using R package 'Vegan' based on OTU-level relative abundance data obtained from bacterial communities in sponge tissues as well as those scraped from agar plates. Canonical (constrained) correspondence analysis (CCA) as implemented in Canoco 5 [254] was used to investigate which experimental variables best explain the variation in species composition regarding OTU-level relative abundance data obtained from the bacterial communities scraped from agar plates. SIMPER [255] was used with respect to square rooted OTU-level relative abundance data to break down the contribution of each OTU to the observed dissimilarity between sample groups. ARB [256] was used to construct a 16S rRNA gene-based phylogenetic tree of bacterial isolates (>800 bp sequences were obtained by Sanger sequencing, see below), their closest type strain (based on BLASTn, bitscore sorted) and the nearest neighbour in the Silva 
guide tree (release 115). The analyses are described in detail in the Supplementary Materials and Methods.

\section{Regrowth, identification and resistance profiling of picked isolates}

Regrowth from glycerol stocks and identification

Isolates stored at $-80{ }^{\circ} \mathrm{C}$ were selected for regrowth based on their specific isolation media via the procedure described in the results section, and subsequently maintained on marine agar if this medium could support their growth. Bacterial cultures were passaged until pure, and their identity was confirmed by 16S rRNA gene Sanger sequencing using the $27 \mathrm{~F}$ and $1492 \mathrm{R}$ general bacterial primers [257]. PCR products were sent to GATC Biotech with sequencing primers 27F, 1492R or 907R [258]. Read ends were trimmed with DNA Baser version 3.5.4.2 until there were $99 \%$ good bases (quality value $>21$ ) in a 20 -base window. For each isolate at minimum the $\mathrm{V} 1$ and $\mathrm{V} 2$ regions of the 16S rRNA gene were sequenced, and the read (after trimming) was required to be at least $800 \mathrm{bp}$ in length. The Sanger read pertaining to an isolate needed to be $>97 \%$ identical to the representative read of the target OTU for it to be considered the corresponding strain. Three attempts for cultivation up to pure culture were made per selected OTU, where possible from distinct wells in the 96-well plates.

Resistance profiling of pure isolates

Isolates were tested for resistance to all antibiotics used in the cultivation experiment (at identical concentrations), as well as for resistance to ampicillin $(50 \mu \mathrm{g} / \mathrm{ml})$ and imipenem $(10 \mu \mathrm{g} / \mathrm{ml})$. The isolates were pregrown in liquid culture (same media but without noble agar), and subsequently inoculated on agar media supplemented with antibiotics. Antibiotic resistance was evaluated three, four and eight days postinoculation while using growth on media without antibiotics as a reference. Bacteria were categorized to be 'resistant', 'intermediately resistant', or 'susceptible'.

\section{Nucleotide sequence accession numbers}

The partial 16S rRNA gene sequences of pure isolates were deposited under accession numbers KP769416 to KP769446. The 16S rRNA gene amplicon sequences were deposited in the ENA SRA database under accession number PRJEB4784 (Table S3.1). 


\section{Results}

\section{Bacterial diversity in sponge samples and their cultivable fraction}

The cultivable bacterial fraction from A. aerophoba, P. ficiformis and C. candelabrum was studied on five media in the form of 60 communities scraped from plates without antibiotics, and in the form of individual isolates picked from media supplemented with (combinations of) different antibiotics (Figure 3.1). Agar media were supplemented with two antibiotics if they predominantly affect the growth of either Gram-negative or Gram-positive bacteria.

In total 679 colonies were picked from plates with antibiotics: 556 (81.9\%) from A. aerophoba, $95(14.0 \%)$ from $P$. ficiformis and $28(4.1 \%)$ from C. candelabrum. Qualified 454-pyrosequencing sequence data yielded 716,939 reads that clustered into 2,282 OTUs. 41,548 reads were assigned to 542 out of 679 picked colonies, a discrepancy that can largely be explained by the fact that 120 red colonies were found to belong to the Penicillium genus (data not shown). Furthermore, 660,721 reads were assigned to the 60 bacterial communities collected by scraping from agar media without antibiotics and 14,670 reads were assigned to the three sponge samples. Table S3.2 shows the number of colonies that were picked or scraped in this experiment sorted according to sponge species and media that were used.

Shannon indices for sponge samples indicated that $C$. candelabrum $\left(\mathrm{H}^{\prime}=3.96\right.$ \pm 0.01 [s.d.]) contained the least diverse microbiota when compared to $A$. aerophoba $\left(\mathrm{H}^{\prime}=5.29 \pm 0.04\right.$ [s.d.]), and P. ficiformis $\left(\mathrm{H}^{\prime}=4.54\right)$. Proteobacteria, Nitrospirae, Chloroflexi, Bacteroidetes, Actinobacteria and Acidobacteria were present in all three sponges at $>0.1 \%$ relative abundance (Figure 3.2). A. aerophoba was distinct because nearly $50 \%$ of the bacteria present belonged to the Chloroflexi. Conversely, the microbiota profiles of $C$. candelabrum and $P$. ficiformis were characterized by high relative abundances of Proteobacteria and Nitrospirae. It is noteworthy that in the microbiota of $P$. ficiformis $>20 \%$ of the bacteria belonged to Bacteroidetes whereas in the other two sponges their relative abundance was $<1 \%$. Rarefaction analysis demonstrated that the current sequencing depth did not capture the complete bacterial diversity in the sponge samples. (Figure S3.1). 


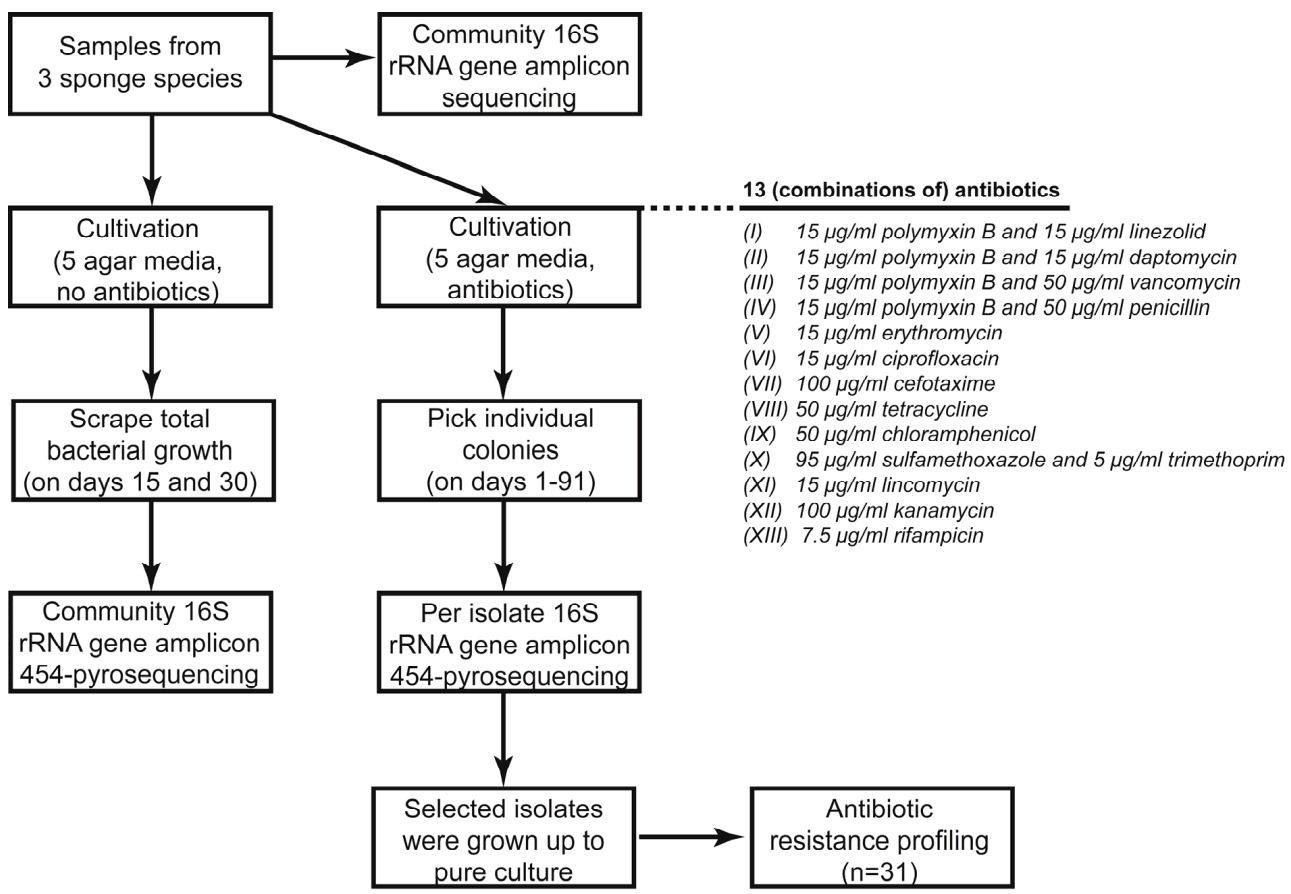

Figure 3.1 Flow diagram of the experiment. Bacterial diversity in samples from three different sponges (A. aerophoba, $P$. ficiformis and C. candelabrum) was compared to their cultivable fraction. Sponge samples were inoculated directly onto agar and on filter membranes on top of the agar. Resistance profiles were determined of 31 different isolates.

A. aerophoba

C. candelabrum

P. ficiformis

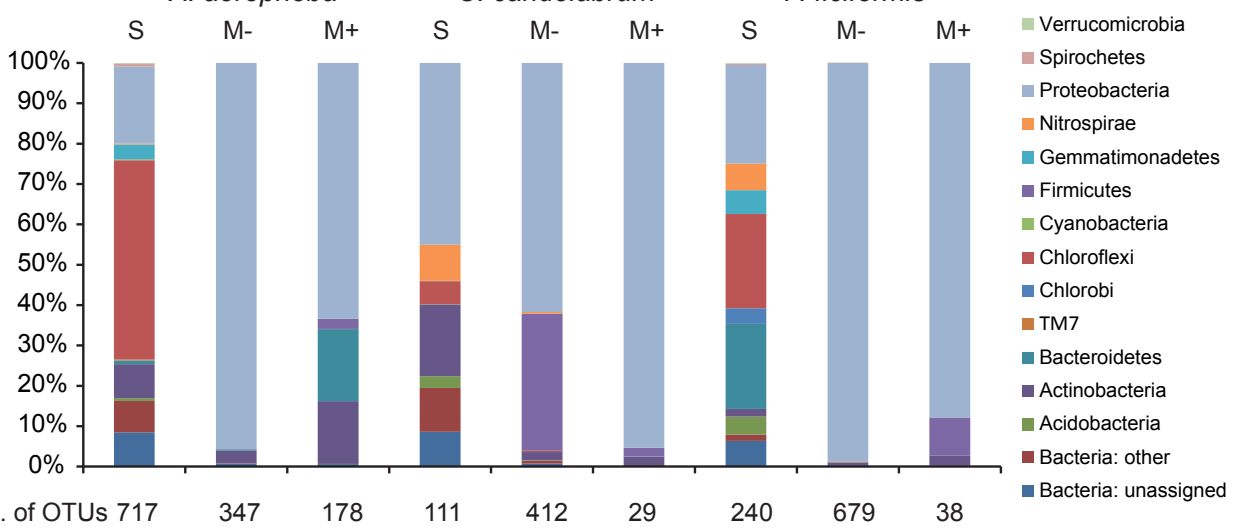

Figure 3.2 Phyla that were detected in the sponge samples (S), on media without antibiotics (M-) and on media with antibiotics $(\mathrm{M}+)$. Phyla with a relative abundance $<0.1 \%$ are not shown. The relative abundance values are based on the combined reads of the different experimental groups.

\section{Bacteria isolated in the absence of antibiotics}

Bacterial communities on the five agar media without antibiotics was dominated by Proteobacteria ( $>50 \%$ relative abundance) for the three sponge species investigated 
(Figure 3.2), which in most cases was attributed to OTU denovo1749 (>50\% relative abundance in 44 out of 60 scraped fractions), an $\alpha$-proteobacterium affiliated to the Pseudovibrio genus. Notably, OTU1749 was not detected in the sponges samples. On plates inoculated with $C$. candelabrum samples also Firmicutes were relatively abundant with $34 \%$ of the total reads.

Hierarchical clustering was performed to investigate dissimilarity at the OTU level between communities present in the sponge samples and those present on media without antibiotics (Figure 3.3). A. aerophoba-derived communities retrieved by scraping from plates and filters formed one large cluster because all samples featured a relatively high abundance of members belonging to the genus Ruegeria (OTU HE574879) (Table S3.3). Within this cluster, as a general rule, higher similarity was observed between samples that were retrieved from the same medium (Figure 3.3). Retrieved communities derived from $P$. ficiformis and $C$. candelabrum formed two mixed branches containing each $>10$ samples for which separate clustering can be ascribed to a relatively high abundance of a subset of 6 specific OTUs in 1 of the 2 branches. These signature OTUs constitute Microbulbifer spp. (5 OTUs) and a Pseudovibrio sp. (1 OTU). Samples within both of these branches showed further grouping according to cultivation medium. The remaining samples in the dendrogram are the original sponge communities and 12 communities derived from $P$. ficiformis and $C$. candelabrum that were highly dissimilar to each other. All except one of these 12 communities were comprised of $<10$ visible colonies.

CCA of OTU-level relative abundance data from the scraped bacterial communities indicated that the explanatory variables medium I (marine agar), medium II (marine agar, 10-fold diluted nutrients), sponge C. candelabrum and medium III (Mueller Hinton agar) could explain respectively 7.0\% ( $p=0.012$ ), 4.5\% ( $p$ $=0.002), 3.9 \%(p=0.002)$ and $2.6 \%(p=0.022)$ of the total variation in diversity using forward selection. Most variation could not be accounted for by the explanatory variables that were evaluated. Similarity percentage analysis (SIMPER) analyses indicated that most dissimilarity in terms of bacterial growth scraped from each sponge species could be explained by different levels of average relative abundance of OTU denovo1749 (Pseudovibrio sp.). In scraped communities derived from $P$. ficiformis the average relative abundance of OTU denovo1749 was $89 \%$, while the corresponding values for $A$. aerophoba and C. candelabrum were $77 \%$ and $63 \%$, respectively. Other top contributors to dissimilarity were OTU HE574879 (Ruegeria 
sp.) that was present with higher relative abundance, and OTU GQ118701 (Microbulbifer sp.) that was present with lower relative abundance, on plates inoculated with material from $A$. aerophoba, as compared to plates inoculated with samples from the other two sponges. In addition, OTU JN579972 (Bacillus sp.) contributed to the dissimilarity by a higher relative abundance on plates inoculated with C. candelabrum. Comparison by SIMPER of bacterial communities retrieved directly from agar and those obtained on filter membranes showed that there are no bacterial taxa that highly favour one growth surface over the other i.e. no taxon occurred with great differences in relative abundance that also substantially $(>2 \%)$ contributed to the dissimilarity.

Bacterial growth on the scraped plates was investigated for novel cultivable bacteria. Seventy-nine novel OTUs ( $\leq 95 \%$ identity of the representative read to the 16S rRNA gene of the closest type strain) that were identified. However, 13 of these 79 OTUs had a lower relative abundance in the scraped communities as compared to the inoculum (Figure S3.2). Therefore, it might be that these OTUs did not grow but instead were derived from sponge material that persisted on the agar media. To address this issue we applied three criteria to conclude bacteria growth: (i) OTUs needed to increase in relative abundance as compared to the inoculum, (ii) OTUs needed to have a relative abundance of $\geq 0.2 \%$, and (iii) the scraped community required $\geq 20$ visible colonies. Adopting these criteria, we retained as many as 27 novel cultivable OTUs from the scraped communities of which the representative read has $\leq 95 \%$ identity to the $16 \mathrm{~S}$ rRNA gene of the closest type strain (Table 3.1). At the phylum-level the 27 OTUs belong to the Proteobacteria (10 OTUs [8 $\alpha$ - and 2 Y-]), Firmicutes (2 OTUs), Bacteroidetes (1 OTU) and Actinobacteria (14 OTUs). Most $(19 / 27)$ novel OTUs grew on Mueller-Hinton-based agar media. Five of the novel OTUs (AJ347026, denovo528, denovo574, GU118526 and denovo98) were detected in the inoculum (sponge sample) as well (Table S3.3).

\section{Bacteria isolated in the presence of antibiotics}

Bacterial colonies were identified by double-barcoded 454-pyrosequencing. Reads were allocated to 542 out of 679 picked colonies, and amongst these colonies a total of 211 OTUs were detected. An OTU was only assigned if it represented $>50 \%$ of reads obtained for that colony, which resulted in 47 OTUs being assigned to 425 picked colonies (Figure S3.3). This assignment strategy was used in order to address 
the high number of OTUs that were detected by only one or a few reads, and in almost all cases comprised the minority of reads assigned to a colony (Figure S3.4). In this way we adopt a conservative approach and limit ourselves to strains from OTUs that are most likely truly represented by growing bacteria and discard potential sequence artefacts, even though OTUs detected by one or a few reads can still be informative about bacterial presence [259]. On average, a similar number of OTUs were assigned to colonies picked directly from agar media (2.56 \pm 2.10 [s.d]) as compared to those picked from filters on top of agar media (2.33 \pm 1.80 [s.d.]). For 31 of 47 OTUs that were assigned to colonies, a representative isolate was successfully regrown from the glycerol stocks up to pure culture. OTU denovo1749 (Pseudovibrio sp.) was assigned to isolates obtained from all three sponge species, and from two sponge species a representative isolate belonging to this OTU was successfully cultured up to pure culture. Isolates are assumed to be different strains if they belong to the same OTU but are obtained from different sponges, and hence both strains were included in subsequent analyses.

For each of the 31 pure cultures the 16S rRNA gene was sequenced to a length of at least $800 \mathrm{bp}$. A phylogenetic tree was constructed (Figure 3.4) based on 16S rRNA gene sequences from the isolates we obtained in pure culture, their most closely related type strain, and their closest neighbour in the Silva guide tree. These isolates belonged to the Firmicutes $(n=5)$, Actinobacteria $(n=6)$, Proteobacteria $(n=15)$ and Bacteroidetes $(n=5)$. The most closely related type strains could be separated into those isolated from the marine environment $(n=15)$, and those isolated mostly from soil, air and plants $(n=12)$. A similar distribution was observed for the closest neighbours where 18 out of 27 sequences originated from the marine environment. Three isolates belonging to the family of Flavobacteriaceae were isolated that potentially constitute two new genera and one new species. These strains exhibited the highest 16S rRNA gene sequence identity to Kriegella aquimaris (denovo1540, 94.9\% identity), Gaetbulibacter marinus (denovo1624, 95.6\% identity) and Lacinutrix algicola (denovo1558, 96.7\% identity). 


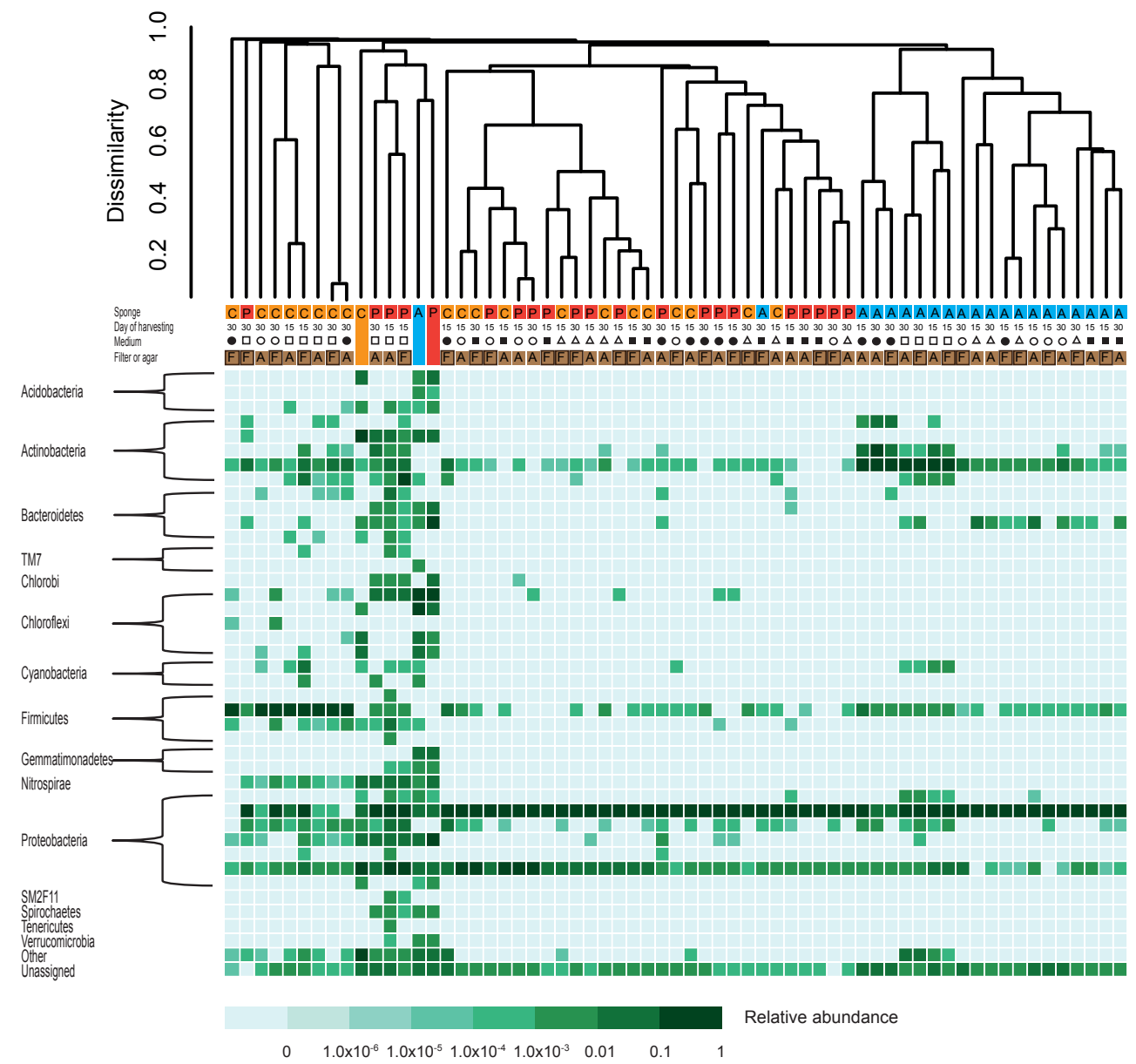

Figure 3.3 Hierarchical clustering using Bray-Curtis distance based on 16S rRNA gene amplicons generated from three sponge samples ( $A$. aerophoba, $P$. ficiformis and $C$. candelabrum) and corresponding communities retrieved by scraping from agar media. Bacterial communities were investigated on marine agar (squares), marine agar 10-fold diluted (open squares), Mufeller-Hinton agar (circles), Mueller-Hinton 10-fold diluted (open circles) and mucin agar (open triangles). Samples were either inoculated in direct contact with agar $(A)$ or on top of a filter $(F)$, and were harvested 15 and 30 days post-inoculation. The sponge samples (inocula) are indicated with continuous colour bars. Hierarchical clustering was performed at the OTU-level (97\% identity clusters). The heatmap corresponds to relative abundance values of order-level phylogenetic groups $(>0.01 \%$ relative abundance in at least one sample). 


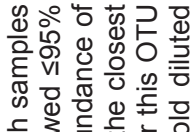

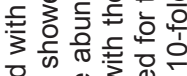

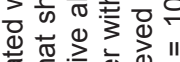

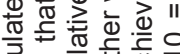

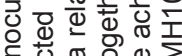

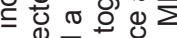

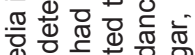

ह

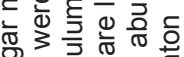

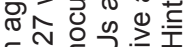

ह

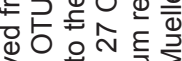

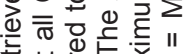

屯苟离离工

ळ

产定 00.

ह่

हो

즌

㐘

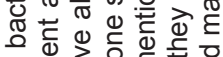

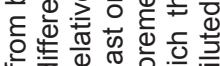

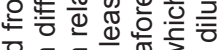

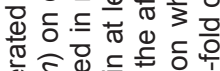

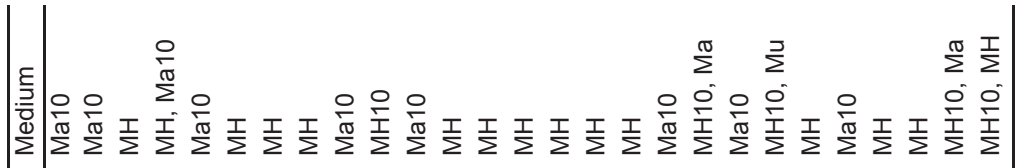

ह

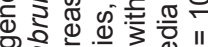

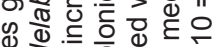

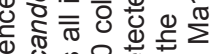

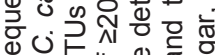

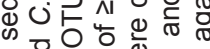

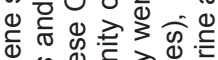
ब.

$\varangle ह F$ है

足过它

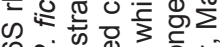

ㄴ.

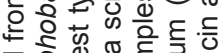

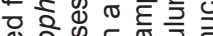

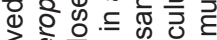
过 ब

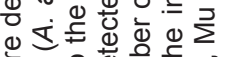

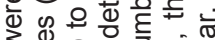

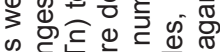

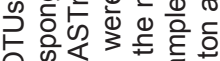

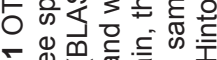
гं山

के

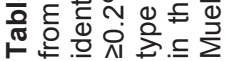

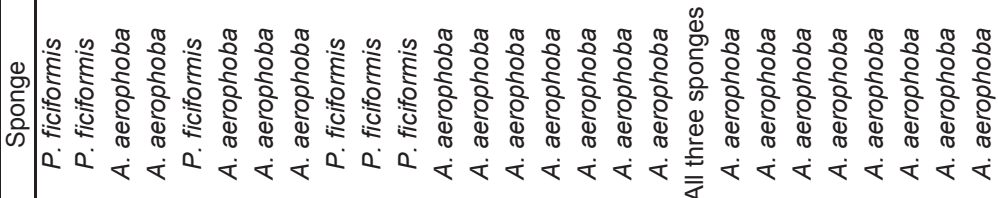

- 華

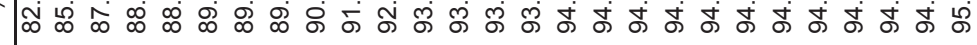

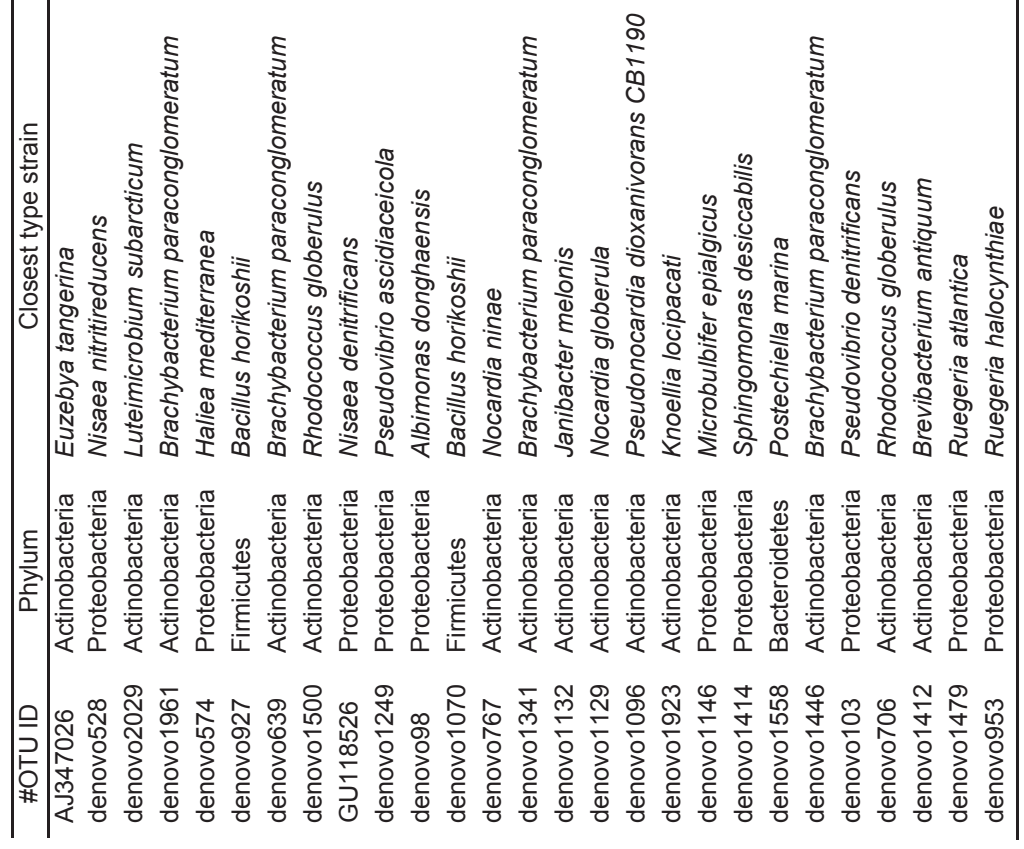


Furthermore, for each of the 31 pure cultures antibiotic resistance was investigated against the antibiotics used in the cultivation experiment, plus ampicillin and imipenem (Table 3.2). Resistance was most frequent against tetracycline, polymyxin $\mathrm{B}$ and kanamycin, whereas no single isolate was resistant to rifampicin or imipenem. Generally, if isolates were susceptible to a certain antibiotic, then the corresponding OTU was not detected on media supplemented with this antibiotic in the original cultivation experiment from which they were derived. Except for Bradyrhizobium pachyrhizi (OTU: FJ624884), isolates were always simultaneously resistant to both antibiotics belonging to the penicillin group of $\beta$-lactam antibiotics i.e. ampicillin and penicillin. Eleven Gram-negative bacteria were sensitive to vancomycin and/or daptomycin, whereas these antibiotics primarily target Gram-positive bacteria (Table 3.2).

Publicly available genomes were obtained for six bacteria of the same species as our isolates and investigated in terms of resistance gene composition (Table S3.4) [105]. We detected putative $\beta$-lactam resistance genes in all tested genomes even though only Acinetobacter radioresistens was found to be resistant to a $\beta$-lactam antibiotic (penicillin). A. radioresistens was also found to be resistant to both chloramphenicol and erythromycin. In each of six publicly available $A$. radioresistens genomes we detected genes encoding a chloramphenicol transferase, membrane fusion protein MacA, and inner membrane protein MacB, which could provide resistance to the aforementioned antibiotics. In three genomes (from Sphinogobium xenophagum, Leisingera aquimarina and Bacillus stratosphericus) MFS efflux pump genes were detected, which could explain why our corresponding isolates are tetracycline-resistant.

\section{Comparison of bacterial presence in sponge samples and their cultivable fraction}

We found that for $P$. ficiformis and C. candelabrum, respectively, 45 and 19 OTUs were detected in the sponge samples that were also present in the scraped fractions (Figure 3.5). In the case of $A$. aerophoba only two OTUs (GU940713 and EU803928) were retrieved in the scraped fraction, and those were classified at the genus level to Propionibacterium and Halomonas. Table S3.5 provides taxonomic information regarding the OTUs that overlap for the different datasets. For all three sponges, an OTU was never detected both in the sponge samples and on media supplemented 
with antibiotics, without it also being detected in communities scraped from media without antibiotics. This is in line with the expectation that the addition of antibiotics and the picking strategy (whereby merely visual growth was considered) prohibits capturing the full diversity of the cultivable fraction on media containing antibiotics.

Eight OTUs were detected in the bacterial communities of all sponge species (Figure 3.5). In addition, 78 OTUs were shared exclusively between $A$. aerophoba and $P$. ficiformis, whereas very few OTUs were shared between either of these two sponges and C. candelabrum (2 and 0 OTUs, respectively). The highest number of OTUs was detected in the combined scraped communities, which could be a product of this experimental group constituting the bulk of the reads (92.2\%) (Table S3.3). 


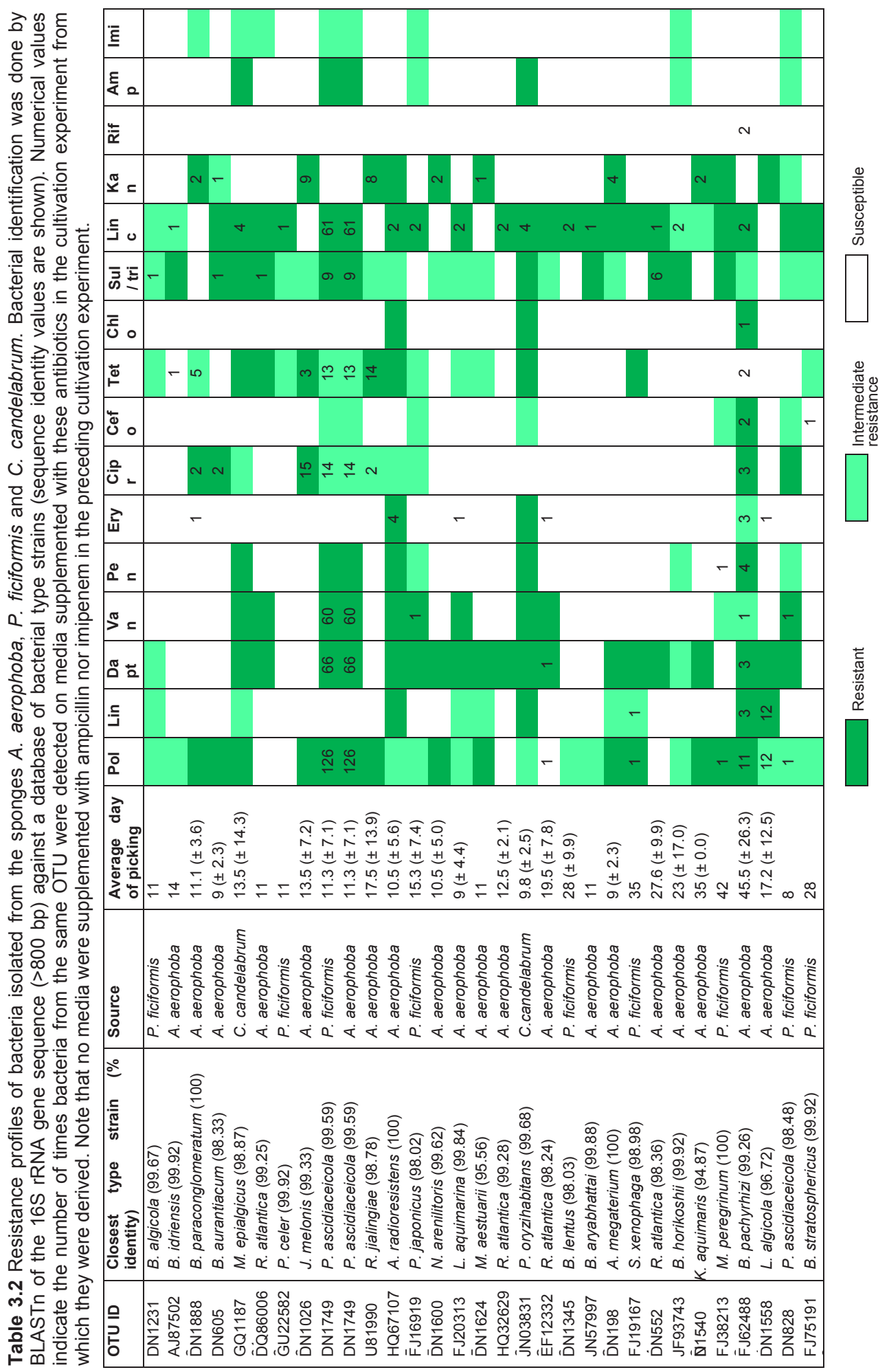




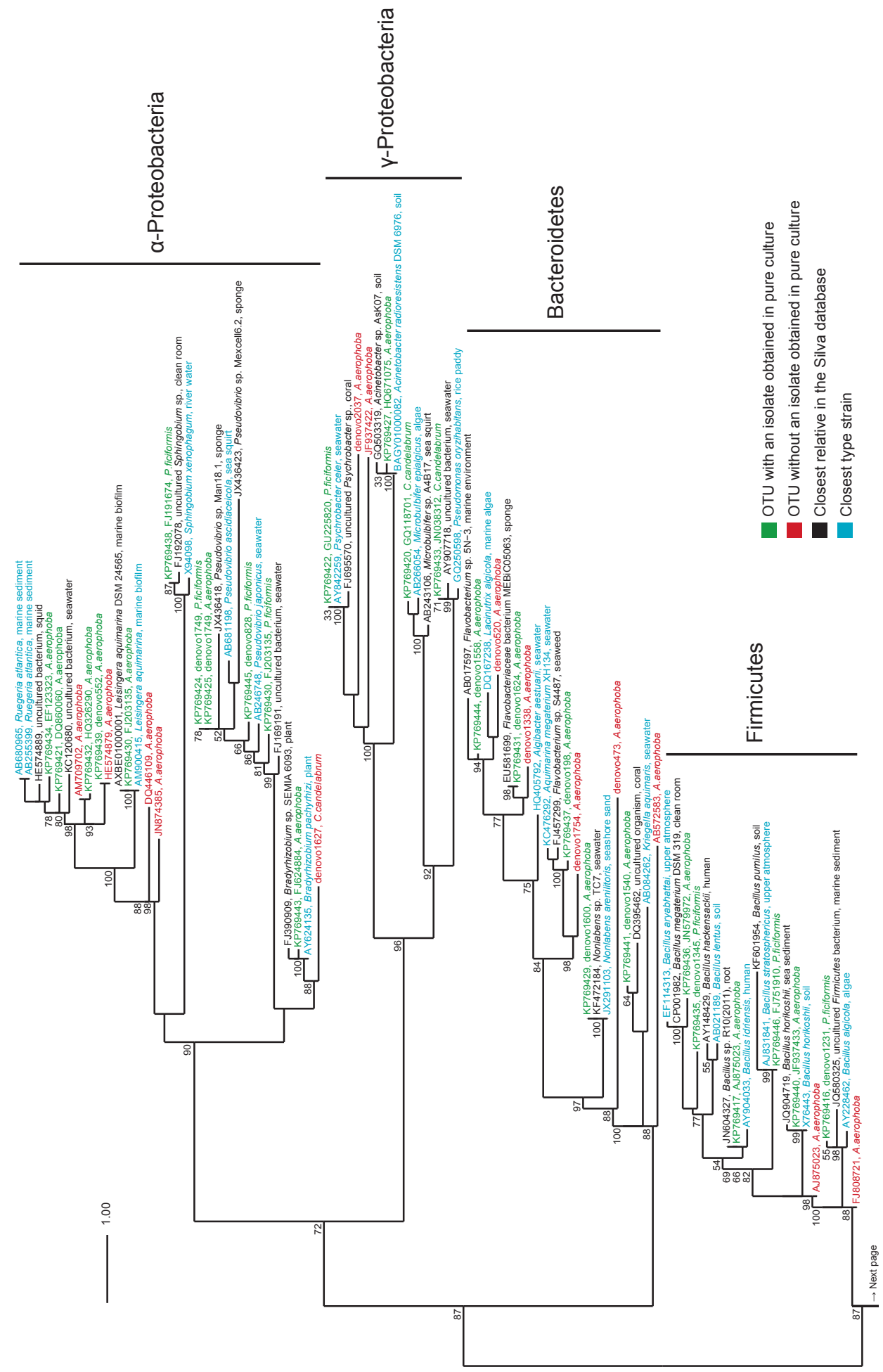




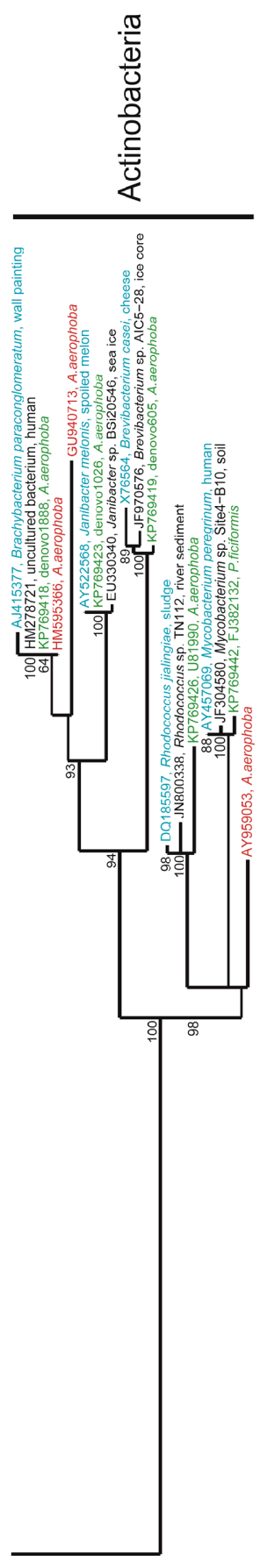

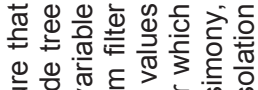

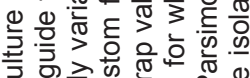

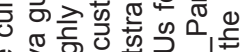

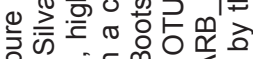

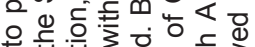

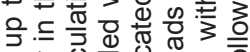

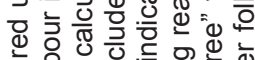

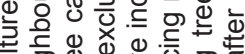

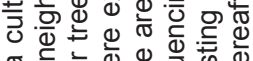

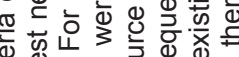
过

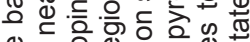

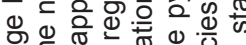

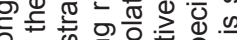
क力 응 is

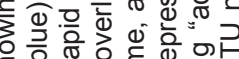

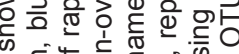
की

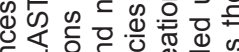

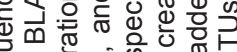

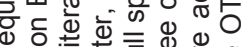

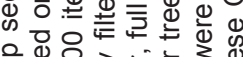

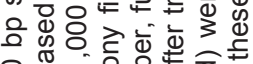

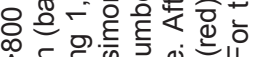
舟. ․ㅜㄴ.

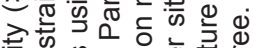

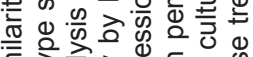

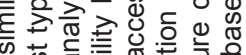

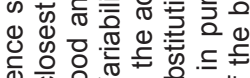

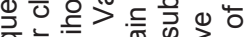

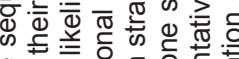

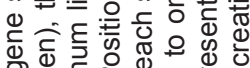
$\circlearrowleft$ 屯 है

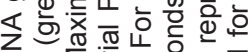

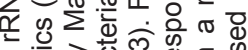

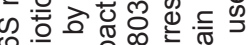
을

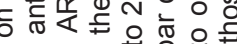

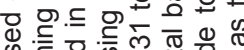
迹. ป ब记

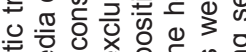

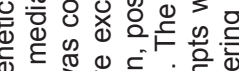
ภำ

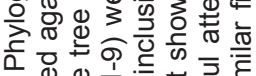

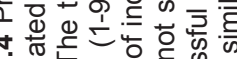

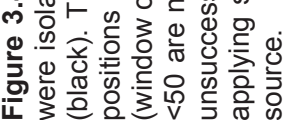


(A)

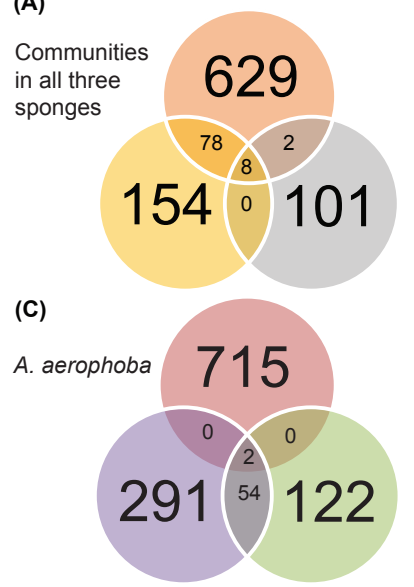

(B)

P. ficiformis

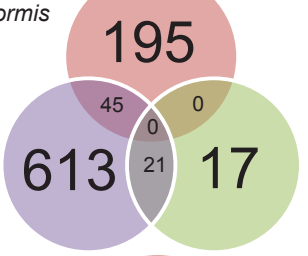

(D)

C. candelabrum

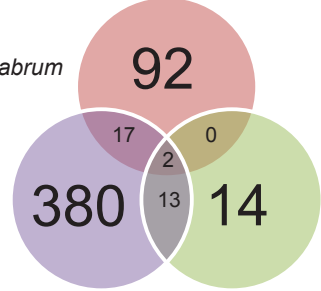

Legend

Community in sponge samples

Bacteria retrieved from media without antibiotics

Colonies picked from media with antibiotics

A. aerophoba

P. ficiformis

C. candelabrum

Figure 3.5 Venn diagrams that illustrate the relationship between OTUs that were detected in the three sponge samples, colonies picked from agar media supplemented with a wide variety of antibiotics, and bacteria retrieved by scraping from agar media without antibiotics. Panel A compares the overlap in terms OTUs in the samples of the three different sponge species ( $A$. aerophoba, $P$. ficiformis and C. candelabrum). Panel B, C and D compare the overlap in terms of OTUs between the sponge samples and their cultivable fractions

\section{Discussion}

\section{Bacterial profiles of the sponge hosts}

In studying bacterial culturability it is essential to know which bacterial species are present in the original samples. Therefore, the bacterial community profiles of the sponges studied here, i.e. A. aerophoba, C. candelabrum and $P$. ficiformis were determined. In the microbiota of Aplysina spp. the sponge-specific candidate phylum Poribacteria was originally discovered [260], of which just recently a member was genomically characterized after single-cell sorting [261, 262]. Poribacteria could not be detected in this study due to insufficient similarity of the 454-pyrosequencing forward primer to the 16S rRNA gene target. Only 8 out of 972 OTUs detected in the sponge samples were shared between all three sponges indicating that, as suggested by past studies [126, 263], bacterial colonization is highly sponge speciesspecific.

\section{Bacterial diversity captured by scraping bacterial biomass from agar media}

Twenty-seven novel OTUs (defined as those for which the representative read has $<95 \%$ identity to the 16 S rRNA gene of the closest type strain) were cultivated, albeit at $\leq 3 \%$ relative abundance. Fourteen out of 27 novel OTUs belong to the phylum Actinobacteria, members of which are known producers of secondary metabolites 
$[125,244,264]$. These strains are therefore particularly interesting for obtaining novel bioactive compounds with potential pharmaceutical applications. Five OTUs that met the criteria for growth were detected of which the 454-pyrosequencing read exhibited $<89 \%$ sequence similarity with the closest type strain, and these strains (3 Actinobacteria and 2 Proteobacteria) potentially belong to novel orders [265]. Three of these five OTUs (OTUs AJ347026, GU118536 and denovo528) were detected in bacterial biomass retrieved from agar media as well as in the original sponge samples, indicating a role in the sponge host. Consequently, they are of particular interest in the study of host-symbiont relations. OTU AJ347026 is especially relevant for these studies since it is highly novel (82.2\% sequence similarity of the 454pyrosequencing read to the 16S rRNA gene of the closest type strain), and it was already previously assigned to a sponge-specific clade [266]. OTU GU118536 was detected earlier in the coral Montastraea faveolata by cultivation-independent methods [267]. We detected no growth of novel Chloroflexi, Nitrospirae and Bacteroidetes, which are typically considered harder to cultivate by conventional plating methodologies such as those applied in this study. Our study is the first cultivation study for $C$. candelabrum, but nevertheless, most novel bacteria were detected in the cultivable fraction from $A$. aerophoba, warranting further investigation into those samples. It should be noted that since we did not obtain strains belonging to the novel OTUs (Table 3.1) in pure culture, there is the possibility that some are dependent on other strains for growth or some compounds from the sponges, and might therefore not be readily cultivable up to pure cultures by the methods applied here [268].

One issue of analysing bacterial growth by scraping from agar media is that cell material from the inoculum can still be present, and as a result bacteria may be detected that did not actually grow. This is an important caveat of the plate scraping method that needs to be considered in growth experiments that use high throughput $16 \mathrm{~S}$ rRNA gene sequencing. We acknowledge that this phenomenon could have occurred in our experiments when in one scraped community that was comprised of only two visible colonies as much as 188 OTUs were detected. In addition, in some scraped communities comprised of $<10$ colonies OTUs were detected that were also present in the sponge samples, but at lower relative abundance. For example, an OTU (DQ889875) that corresponded to a novel $\delta$-proteobacterium was present with $12 \%$ relative abundance in the $P$. ficiformis sample, whereas it was present at a 
relative abundance of $2.7 \%$ in a $P$. ficiformis scraped community that consisted of 2 colonies. We assume that this bacterium did not grow, even though we cannot exclude the possibility that micro-colonies appeared that were invisible to the naked eye. In order to address this issue we have applied conservative criteria before concluding bacterial growth. A separate issue is that glycerol-frozen sponge samples were used and as such bacteria that do not survive the freeze-thawing process are not recovered.

\section{Bacterial diversity captured by picking of individual isolates}

Bacterial sponge isolates typically belong to the Actinobacteria, Bacteroidetes, Firmicutes or $\alpha$-, $\beta$ - and $\gamma$-Proteobacteria [148, 269]. Here, each of 47 isolates obtained from media supplemented with antibiotics belongs to these four phyla, and only in two cases the bacterium (or a similar strain from the same OTU) was also detected in the original sponge sample. OTU1749 was assigned to 223 colonies even though in the sponges samples this OTU was not detected, suggesting that Pseudovibrio spp. have a strong growth advantage on the applied cultivation media. Pseudovibrio spp. have been repeatedly isolated from marine sponges without being detected by cultivation-independent techniques $[150,270]$. These results imply that in sponges a vast number of different bacterial species reside that are not detected at read depths of at least 3,000-7,000 reads. Therefore, the presence of additional uncovered bacterial diversity is still to be expected.

To isolate a diverse range of bacteria we also applied a polycarbonate filter that could mimic the inner structures of the filter-feeding sponge, an extended incubation period, poor and selective media, and supplementation of antibiotics. We found that the application of a polycarbonate filter did not complement conventional plating with respect to retrieved bacterial diversity. Two bacterial strains were picked no earlier than 34 days post-incubation. These were a Mycobacterium peregrinum (OTU FJ382132) and a bacterium that potentially belongs to a new genus in the Flavobacteriaceae family (OTU denov01540). After transfer to fresh media visible colonies grew after a week, which indicates that their initially slower growth might have been hampered due to adjustment to in vitro conditions. The Flavobacteriaceae sp. was originally isolated on mucin agar, but was also found to grow on marine agar. Two other strains, namely a Bradyrhizobium pachyrhizi (OTU FJ624884) and another novel Flavobacteriaceae sp. (OTU denovo1558), were isolated on 10-fold diluted 
marine agar and mucin agar, respectively, and both were unable to grow on (undiluted) marine agar. Lastly, on marine agar, a third novel Flavobacteriaceae sp. (OTU denov01624) was isolated which potentially constitutes a new genus. These results demonstrate that longer incubation periods and a spectrum of different media were effective to capture a broader diversity of bacteria. Pseudovibrio spp. hindered the isolation of diverse bacteria by virtue of their fast (over-)growth. Bacteria from this genus often dominate cultured fractions of sponge-associated bacterial communities [148, 271]. Pseudovibrio spp. were sensitive to kanamycin, which aided in the isolation of four different bacterial strains from agar media supplemented with this antibiotic that were not retrieved from media supplemented with other antibiotics. In general, these results show that refinement of standard cultivation strategies, such as supplementation of media with antibiotics, can be used to acquire yet uncovered bacterial diversity. The success at acquisition of previously uncultivated bacteria is also related to sponges being relatively unexplored [93, 129, 271].

\section{Sponge bacteria as a reservoir for antibiotic resistance genes}

Resistance genes have been detected in all biological niches associated with complex microbial communities [97]. It is therefore hypothesized that also spongeassociated bacteria harbour resistance genes that could (eventually) become available to human pathogens [272]. The bacteria isolated in this study were resistant to a diverse range of antibiotics that are routinely used in the clinic. Publicly available genomes of a subset of these species indicated the potential presence of resistance genes against tetracyclines, $\beta$-lactams, aminoglycosides, chloramphenicol and erythromycin, frequently correlating with the observed resistance phenotypes of our corresponding strains. However, class A or B $\beta$-lactamase resistance genes were detected in all investigated public genomes even though resistance to ampicillin and/or penicillin was only observed in 1 out of 6 of our corresponding strains. Functional (meta-)genomic studies are suggested to identify mechanisms behind the observed antibiotic resistance, and to investigate if sponges are a reservoir for (novel) antibiotic resistance genes. 


\section{Conclusion}

Using high throughput cultivation we captured a breadth of previously uncultured sponge-associated bacteria, mainly from $A$. aerophoba. At the same time, we presented criteria to address an important caveat of the plate scraping method whereby bacteria may be detected that did not actually grow. We propose 27 OTUs that are of prime interest due to their novelty alone. Among these, a diverse range of novel Actinobacteria was cultivated, which shows that previously uncultivated bacteria with high biotechnological and pharmaceutical potential are still accessible by classic cultivation. One highly novel previously uncultured Actinobacterium was cultivated that was also detected in the original sponge sample. This strain appears to belong to a sponge-specific clade, which makes it particularly interesting in the study of host-symbiont relations. In addition, three Flavobacteriaceae spp. were cultured up to pure cultures and potentially constitute two new genera and one new species.

\section{Acknowledgements}

This work was supported by the European Union through the EvoTAR project (Grant agreement no. 282004) and the Dutch BE-BASIC foundation (project 7.1.5). 


\section{Supplementary information}

\section{Supplementary Tables and Figures}

Tables S3.1, S3.3, S3.4 and S3.5 are available upon request.

Table S3.2 Panel A: number of colonies picked from samples of three sponges $(A$. aerophoba, $C$. candelabrum and $P$. ficiformis) inoculated on five different agar media supplemented with antibiotics. Panel B and C: number of visible colonies scraped from identical samples inoculated on media without antibiotics. Cell material was harvested 15 and 30 days post-inoculation.

(A)

\begin{tabular}{|l|l|l|l|l|l|l|r|}
\hline & Aplysina aerophoba & & \multicolumn{3}{l|}{ Corticium candelabrum } & \multicolumn{3}{|l|}{ Petrosia ficiformis } \\
\cline { 2 - 8 } & Agar & Filter & Agar & Filter & \multicolumn{2}{|l}{ Agar } & Filter \\
\hline Marine agar & 110 & 53 & 3 & & 4 & 21 & 11 \\
\hline Marine agar (10 X diluted) & 60 & 31 & 18 & & 1 & 1 & 0 \\
\hline Mueller-Hinton agar & 51 & 33 & 1 & & 0 & 12 & 10 \\
\hline Mueller-Hinton agar (10 X diluted) & 78 & 31 & 1 & & 0 & 18 & 4 \\
\hline Mucin agar & 57 & 52 & 0 & & 0 & 16 & 2 \\
\hline
\end{tabular}

(B)

\begin{tabular}{|l|l|l|l|l|l|r|r|}
\hline Material from 3 plates scraped at day 15 & \multicolumn{2}{|l|}{ Aplysina aerophoba } & \multicolumn{2}{l|}{ Corticium candelabrum } & \multicolumn{2}{l|}{ Petrosia ficiformis } \\
\cline { 2 - 8 } & Agar & Filter & Agar & Filter & \multicolumn{2}{|l}{ Agar } & Filter \\
\hline Marine agar & 174 & 55 & 17 & & 14 & 39 & 24 \\
\hline Marine agar (10 X diluted) & 195 & 160 & 4 & 3 & 2 & 2 \\
\hline Mueller-Hinton agar & 101 & 37 & 6 & 4 & 11 & 6 \\
\hline Mueller-Hinton agar (10 X diluted) & 226 & 104 & 4 & 4 & 19 & 17 \\
\hline Mucin agar & 183 & 100 & 93 & & 15 & 18 & 15 \\
\hline
\end{tabular}

(C)

\begin{tabular}{|c|c|c|c|c|c|c|}
\hline \multirow{2}{*}{ Material from 3 plates scraped at day 30} & \multirow{2}{*}{\begin{tabular}{|l|} 
Aplysina aerophoba \\
Agar
\end{tabular}} & \multirow[b]{2}{*}{ Filter } & \multicolumn{2}{|c|}{ Corticium candelabrum } & \multicolumn{2}{|c|}{ Petrosia ficiformis } \\
\hline & & & Agar & Filter & Agar & Filter \\
\hline Marine agar & 252 & 84 & 20 & 14 & 38 & 14 \\
\hline Marine agar (10 X diluted) & 250 & 90 & 7 & 2 & 23 & \\
\hline Mueller-Hinton agar & 133 & 35 & 1 & 4 & 11 & 9 \\
\hline Mueller-Hinton agar (10 X diluted) & 286 & 91 & 3 & 10 & 28 & 16 \\
\hline Mucin agar & 169 & 119 & 93 & 253 & 25 & 14 \\
\hline
\end{tabular}




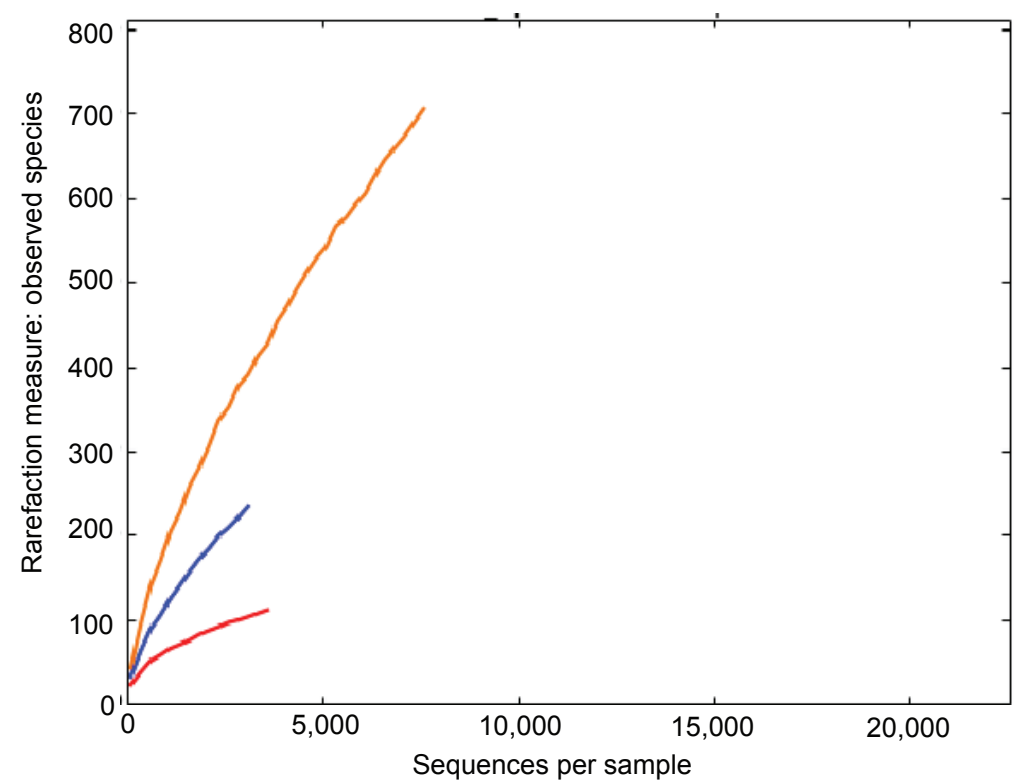

Figure S3.1 Rarefaction curves in which is plotted the number of OTUs detected in samples from the sponges $A$. aerophoba (yellow), $P$. ficiformis (blue) and C. candelabrum (red) as a function of the number of reads sampled.

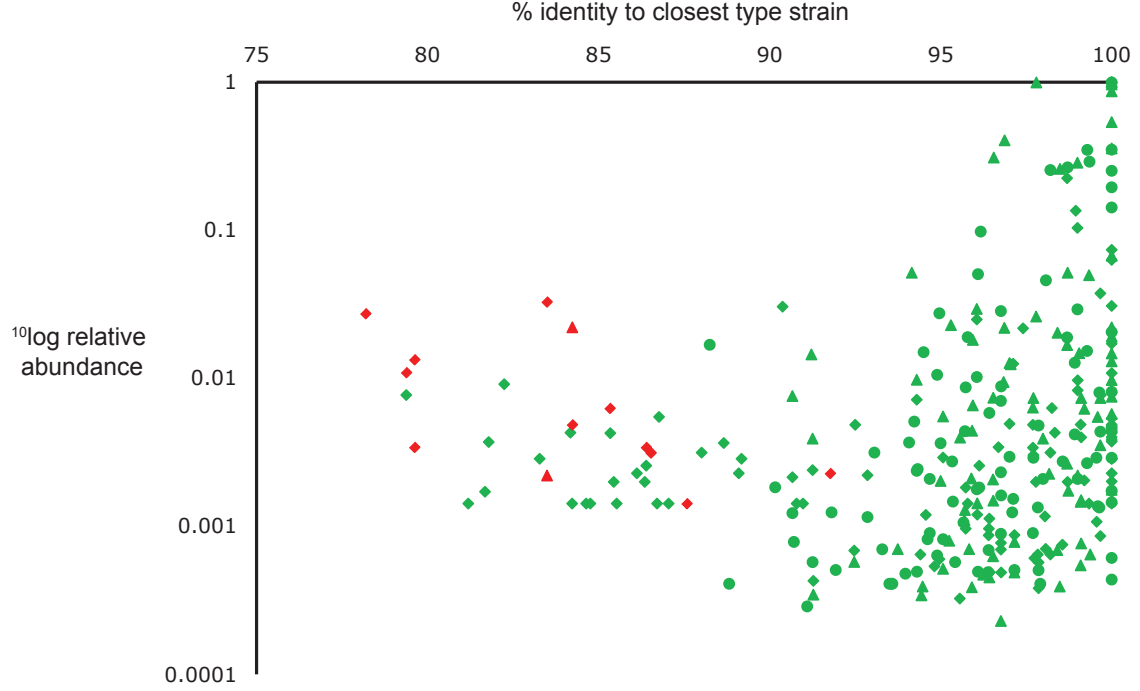

Figure S3.2 The relative abundance values of OTUs that were detected with $>4$ reads in the communities scraped from agar media are plotted against the identity of the corresponding read to the closest type strain, as determined by BLASTn. The highest relative abundance value is shown if the OTU was detected in multiple scraped communities. The OTUs are shown separately for each of the three sponges: A. aerophoba (circles), P. ficiformis (diamonds) and C. candelabrum (triangles). Data points are marked green if the relative abundance of the OTU in the scraped fraction is higher than in the in the sponge sample (the inoculum) or in red if the converse is true. 


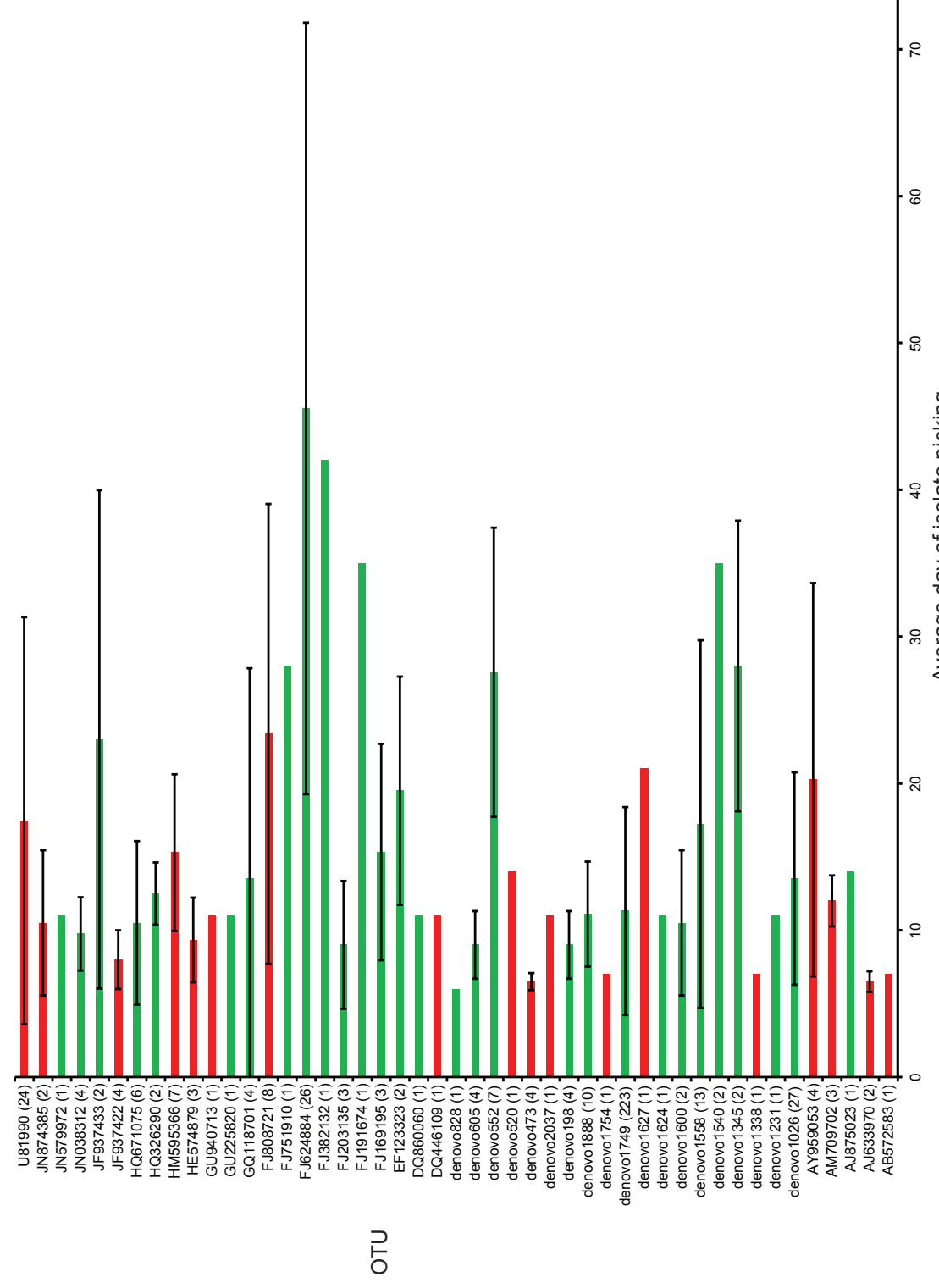

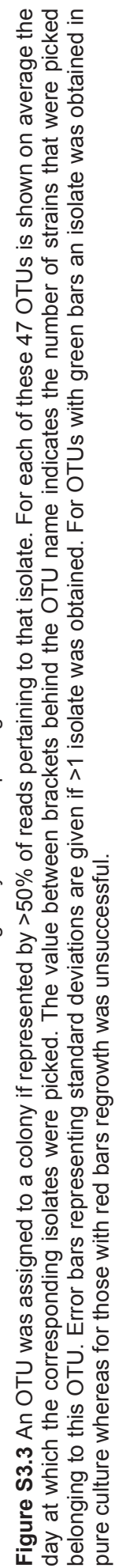




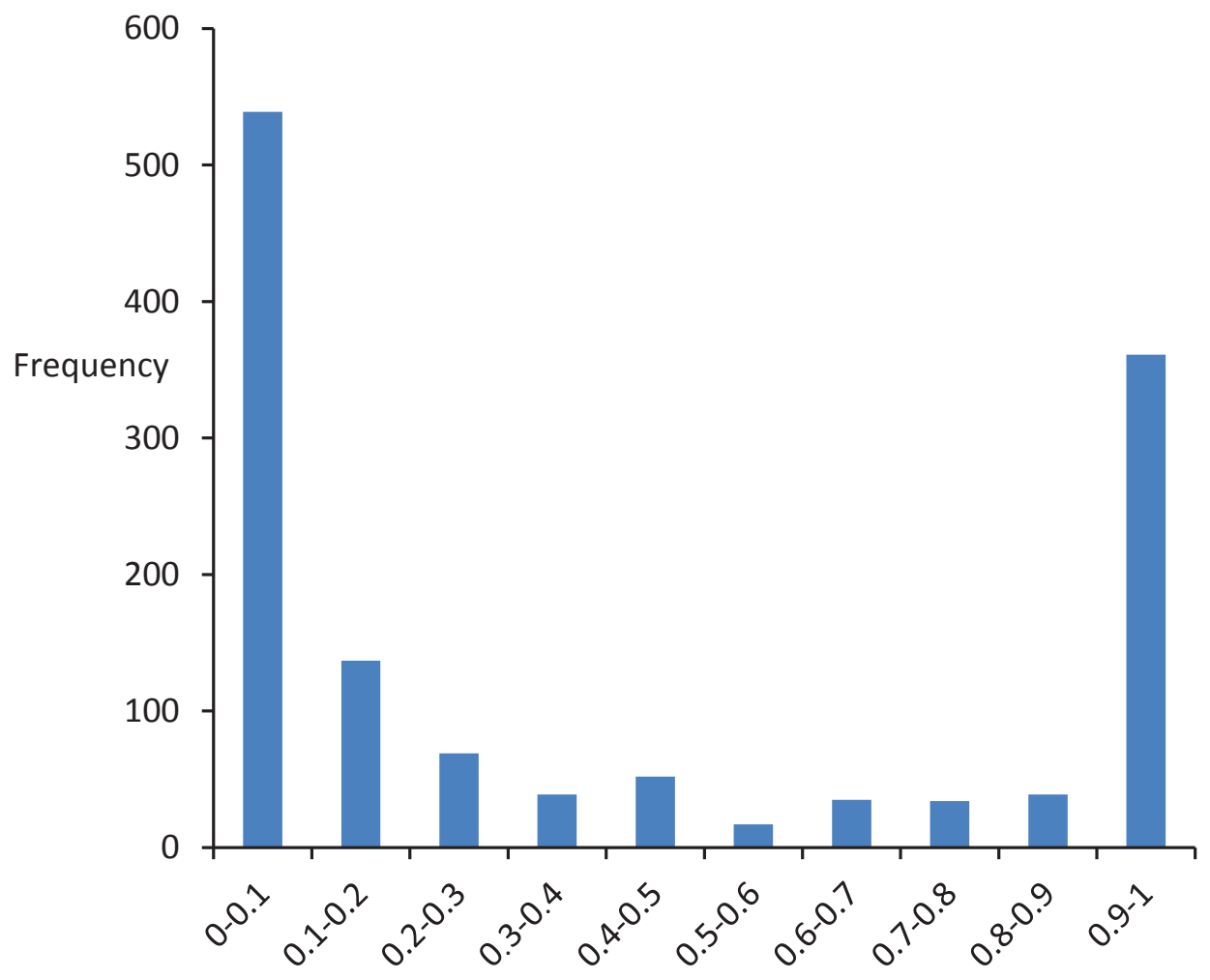

Relative abundance

Figure S3.4 For each OTU assigned to a picked colony, its relative abundance was calculated by dividing the number of reads with which this OTU was detected by the total number of reads assigned to the colony. By this manner a list of relative abundance values was generated, and the distribution of these values is shown here. Note that a high number OTUs were assigned to colonies with $<10 \%$ relative abundance (in most cases these OTUs are detected by one read only), which are expected to be mostly sequencing artefacts.

\section{Supplementary Materials and Methods}

\section{Barcoded 16S rRNA gene amplification and library preparation}

Barcodes were included to facilitate parallel processing of multiple samples. PCRs were performed in a total volume of $100 \mu$ l containing $20 \mu \mathrm{l}$ X Phusion Green HF buffer (Thermo Scientific), $2 \mu \mathrm{l} 10 \mathrm{mM}$ dNTPs, $5 \mu \mathrm{l} 10 \mu \mathrm{M}$ forward primer, $5 \mu \mathrm{l} 10 \mu \mathrm{M}$ reverse primer, $1 \mu \mathrm{l}$ Phusion Hot start II DNA polymerase (2 U/ $\mu \mathrm{l}$, Thermo Scientific) and $2 \mu$ sample $(10-20 \mathrm{ng} / \mu \mathrm{l})$. The PCR program consisted of: initial denaturation of $30 \mathrm{~s}$ at $98^{\circ} \mathrm{C} ; 30$ cycles of denaturation at $98^{\circ} \mathrm{C}$ for $10 \mathrm{~s}$, annealing at $56^{\circ} \mathrm{C}$ for $20 \mathrm{~s}$, and extension at $72{ }^{\circ} \mathrm{C}$ for $20 \mathrm{~s}$; and final extension at $72{ }^{\circ} \mathrm{C}$ for $10 \mathrm{~min}$. At this point 
the identification of picked colonies required an additional step wherein PCR products with identical barcodes in the forward primer were pooled equimolarly (at maximum 48 different barcodes in the reverse primer were combined with a barcode in the forward primer). Either 5, 10 or $20 \mu \mathrm{l}$ of PCR product was included in the pool, depending on agarose gel band intensity. Subsequently (pools of) PCR products were purified with the GeneJET PCR Purification Kit (Thermo Scientific) according to manufacturers' instructions. Isopropanol was included in the binding buffer and elution buffer was re-applied to the column to increase the DNA yield. The DNA concentration of the elute was measured with the Qubit $\AA 2.0$ Fluorometer (Invitrogen) using the Qubit $\AA$ dsDNA BR assay according to manufacturers' instructions. An equimolar mixture of at maximum 54 purified PCR products containing different forward barcodes was prepared and run on a $1 \%$ agarose gel. The gel band at $\sim 425$ bp was cut from the gel and purified using the DNA Gel Extraction Kit (Millipore) according to manufacturers' instructions. The DNA concentration of the mixture was measured by Qubit ${ }^{8} 2.0$ and sent for 454pyrosequencing at GATC Biotech (Konstanz, Germany) on a Roche GS FLX.

\section{Diversity and clustering analyses of bacterial communities}

\section{Rarefactions curves}

For sponge bacterial communities, rarefaction curves were calculated with QIIME scripts [251] applying 10 iterations per subsampling of the OTU table. Subsamples were generated beginning with 100 reads, plus an additional 250 reads were added per subsampling step. QIIME was also used to calculate Shannon diversity metrics. Therefore, the number of reads in the OTU table per sample was rarefied using 1,000 iterations to match the sample with the smallest number of reads. The Shannon diversity metric was calculated for each of the 1,000 rarefied OTU tables and used to calculate an average value per sample.

\section{Hierarchical clustering}

Hierarchical clustering was performed using R package 'Vegan' based on OTU-level relative abundance data obtained from bacterial communities in sponge tissues as well as those scraped from agar plates. Firstly, a matrix of Bray-Curtis dissimilarity values was created with Vegan function 'vegdist'. Thereafter, the dissimilarity matrix was used for average hierarchical clustering with hclust, and finally, a dendrogram was created using the function 'as.dendrogram'. 


\section{Canonical (constrained) correspondence analysis}

Canonical (constrained) correspondence analysis as implemented in Canoco 5 [254] was used to investigate which experimental variables best explain the variation in species composition regarding OTU-level relative abundance data obtained from the bacterial communities scraped from agar plates. Interactive-forward-selection analysis was selected with 'sponge species', 'day of harvesting', 'medium' and 'growth surface' (agar or filter) as explanatory variables. Explanatory variables at the forward selection step were included in order of contribution to the explained variation until the value of the adjusted $\mathrm{R}^{2}$ including the current best candidate exceeded the threshold based on a model with all predictors.

\section{SIMPER}

SIMPER analysis [255] was used with respect to square rooted OTU-level relative abundance data to break down the contribution of each OTU to the observed dissimilarity between sample groups. Pairwise comparisons were performed between samples that were grouped based on sponge species, day of harvesting, medium and growth surface.

\section{Phylogenetic tree construction}

The tree was constructed in ARB [256] by Maximum likelihood analysis using 1,000 iterations of rapid bootstrapping. For tree calculation, highly variable positions (1-9) were excluded using the bacterial Positional Variability by Parsimony filter, and nonoverlapping regions were excluded (window of inclusion, position 5,331 to 26,803 ). After tree construction, representative pyrosequencing reads belonging to OTUs for which unsuccessful attempts were made to obtain a member in pure culture were added using "add species to existing tree" with ARB_Parsimony, applying similar filtering settings as those used for the base tree. 



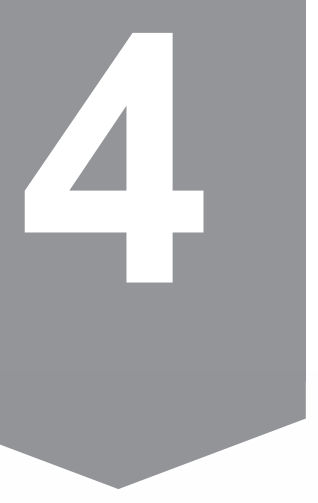

Sponge microbiota are a reservoir of

functional antibiotic resistance genes

Dennis Versluis, Mari Rodriguez de Evgrafov, Morten O.A. Sommer, Detmer Sipkema, Hauke Smidt, Mark W.J. van Passel 


\section{Abstract}

Wide application of antibiotics has contributed to the evolution of multi-drug resistant human pathogens, resulting in poorer treatment outcomes for infections. In the marine environment sea water samples have been investigated as a resistance reservoir; however, no studies have methodically examined sponges as a reservoir of antibiotic resistance. Sponges could be important in this respect because they often contain diverse microbial communities that have the capacity to produce bioactive metabolites. Here, we apply functional metagenomics to study the presence and diversity of functional resistance genes in the sponges Aplysina aerophoba, Petrosia ficiformis and Corticium candelabrum. We obtained 37 insert sequences facilitating resistance to D-cycloserine $(n=6)$, gentamicin $(n=1)$, amikacin $(n=7)$, trimethoprim $(n=17)$, chloramphenicol $(n=1)$, rifampicin $(n=2)$ and ampicillin $(n=3)$. Fifteen of 37 inserts harboured resistance genes that shared $<90 \%$ amino acid identity with known gene products, whereas on 13 inserts no resistance gene could be identified with high confidence, in which case we predicted resistance to be mainly mediated by antibiotic efflux. One marine-specific ampicillin-resistance-conferring $\beta$-lactamase was identified in the genus Pseudovibrio with $41 \%$ global amino acid identity to the closest $\beta$-lactamase with demonstrated functionality, and subsequently classified into a new family termed PSV. Taken together, our results confirm that sponge microbiota host diverse and novel resistance genes that may be harnessed phylogenetically distinct bacteria. 


\section{Introduction}

In the last decades the massive medical and veterinary use of antibiotics has contributed to the selection of multi-drug resistant human pathogens, resulting in poorer treatment outcomes upon infection [273]. Bacterial pathogens can evolve resistance vertically [274] but mainly acquire resistance through horizontal gene transfer [275], where resistance genes are obtained either from the indigenous human microbiota or from environmental microorganisms to which an individual is exposed (e.g. via food, water or soil). The current complement of resistance genes encountered in the human microbiota is suggested to have originated from natural environments, likely in part selected by prior anthropogenic antibiotic pressure [4, 104, 212]. However, microbiota from isolated human populations have also been found to carry functional antibiotic resistance (AR) genes [179]. Soil has been implicated to be a key environmental reservoir of AR genes, as evidenced by the fact that $A R$ genes with perfect nucleotide identity to those in human pathogens have been described in soil-dwelling bacteria [43].

In addition to soil, resistance genes have been found in a broad range of environments that contain complex microbial communities such as activated sludge [98, 99], caves [108], glaciers [191], rivers [103] and animals [100, 101]. As a result, all these environments, and probably many more, can act as potential reservoirs for resistance gene dissemination. It has been shown that $A R$ is a natural phenomenon that predates the modern selective pressure of clinical antibiotic use [109], although little is known about the exact roles of these genes in their native environments [19]. The marine environment is a major genetic reservoir of $A R$, which despite its tremendous bacterial diversity has been little studied in the context of AR gene dissemination [190]. A recent study has identified a range of unclassified resistance genes in ocean water, thereby highlighting its importance as an environmental reservoir [102].

Marine sponges are an ancient lineage of sessile filter-feeding metazoans. Many sponges harbour a dense and diverse microbiota comprising up to $40 \%$ of the total sponge volume, and as such, house a complex ecosystem characterized by host-microbe and microbe-microbe interactions [123]. Much sponge research has focused on the sponge microbiota as a source of novel bioactive compounds [276], whereas its role as a reservoir of AR genes has received limited attention. Still, the 
fact that sponge microbiota can produce diverse antimicrobials [134, 277] would suggest the presence of AR genes. An example of antimicrobials produced by sponge bacteria are rifampicin antibiotics, which at first were only known to be produced by soil Actinobacteria [278, 279], but more recently have also been found to be produced by a sponge-associated Actinobacterium sp. [280].

To date, research on functional AR genes in sponge microbiota has been limited to a Bacillus sp. isolated from the sponge Haliclona simulans. The Bacillus sp. was found to contain two small plasmids, one of which harboured the tetL tetracycline resistance gene [139] whereas the other harboured the ermT erythromycin resistance gene [140]. The plasmid with the tetL gene was shown to be nearly identical to three other tetracycline resistance plasmids identified in the honey bee pathogen Paenibacilus larvae pMA67, in the cheese-resident Lactobacillus sakei Rits 9, and in Sporosarcina urea pSU1 isolated from soil beneath a chicken farm [139]. Furthermore, a TEM $\beta$-lactamase encoding gene was detected in a metatranscriptome dataset from the sponge Crambe crambe (Chapter 2 of this thesis), and the mecA, mupA, qnrB and tetL resistance genes were detected in the sponge Petromica citrina by PCR with gene-specific primers [142]. Based on these results it is tempting to speculate that the sponge microbiota indeed might act as a reservoir of functional AR genes.

The aim of this study was to systematically assess sponge microbiota as a reservoir of AR genes. We screened for functional resistance genes against 14 clinically relevant antibiotics in three Mediterranean high-microbial-abundance sponges, namely Aplysina aerophoba, Petrosia ficiformis and Corticium candelabrum. Small-insert libraries were prepared in Escherichia coli with DNA isolated from sponge tissue of the three sponges (environmental DNA libraries), as well as from pooled DNA isolated from 31 bacterial isolates that were obtained from these sponges (sponge isolates DNA library). The 31 bacterial isolates were obtained from agar media supplemented with antibiotics as part of a previous high-throughput cultivation study (Chapter 3 ).

\section{Materials and methods}

\section{Resistance profiling of bacterial isolates}

One small-insert library was prepared from genomic DNA obtained from 31 bacterial strains (Table 4.1) isolated from the sponges $A$. aerophoba, C. candelabrum and $P$. 
ficiformis. Resistance of these isolates to polymyxin B, erythromycin, ciprofloxacin, cefotaxime, tetracycline, chloramphenicol, rifampicin, ampicillin and imipenem was investigated in a previous study (Chapter 3). Additionally, in this study, resistance of the isolates was tested to gentamicin $(50 \mu \mathrm{g} / \mathrm{ml})$, D-cycloserine $(50 \mu \mathrm{g} / \mathrm{ml})$, chlortetracycline $(50 \mu \mathrm{g} / \mathrm{ml})$, amikacin $(100 \mu \mathrm{g} / \mathrm{ml})$ and trimethoprim $(50 \mu \mathrm{g} / \mathrm{ml})$.

\section{Preparation of linearized vector pZE21}

An E. coli TOP10 strain harbouring the plasmid pZE21 was inoculated into $10 \mathrm{ml}$ LB broth with kanamycin $(50 \mu \mathrm{g} / \mathrm{ml})$. The culture was incubated at $37{ }^{\circ} \mathrm{C}$ overnight. Plasmid isolation was performed with the GeneJET Plasmid Miniprep Kit (Thermo Fisher Scientific, Waltham, United States). The DNA concentration was measured by Qubit $^{\circledR}$ (Thermo Fisher Scientific), and the isolated plasmid was electrophoresed on a $1 \%$ agarose gel for visual inspection. Next, the plasmid was cut by Hincll \#R0103C (NEB, Ipswich, Massachusetts) according to manufacturer's instructions. Subsequently, the plasmid ends were dephosphorylated according to the Protocol for dephosphorylation of 5'-ends of DNA by rSAP (NEB). Finally, the linearized and dephosphorylated plasmid DNA was purified with the Clean-up Concentrator kit (A\&A Biotechnology, Gdynia, Poland).

\section{Preparation of small-insert libraries}

To prepare a small-insert library with a mixture of genomic DNA from 31 pure culture isolates (Table 4.1), we individually isolated DNA with the MasterPure ${ }^{\mathrm{TM}}$ DNA Purification Kit (Epicentre, Madison, Wisconsin). The DNA concentration was measured by Qubit $^{\circledR} 2.0$, and DNA from the different isolates was then pooled at equivalent mass. DNA from sponge tissue samples was isolated with the DNeasy Blood \& Tissue Kit (Qiagen, Hilden, Germany). DNA was fragmented by the E210 sonicator (Covaris, Woburn, Massachusetts) where default operating conditions were used to achieve an average fragment size of $2 \mathrm{kbp}$. The fragmented DNA was electrophoresed on a $1 \%$ agarose gel, and DNA ranging from 0.7-5 kbp was purified by using the GeneJet Gel Extraction Kit (Thermo Fisher Scientific). In the final gel purification step, DNA was eluted with nuclease-free water instead of elution buffer. Subsequently the DNA ends were repaired with the NEBNext ${ }^{\circledR}$ End Repair Module (NEB) according to manufacturer's instructions. The end-repaired DNA was cleaned with the Clean-up Concentrator kit (A\&A Biotechnology), and elution was performed with nuclease-free water. The TaKaRa MightyMix DNA ligation kit (Clontech 
Laboratories Inc., Mountain View, United States) was used for ligation of the endrepaired fragments into the linearized pZE21 vector. Each ligation mixture consisted of $2 \mu \mathrm{l}$ Ligation Mix and $2 \mu \mathrm{l}$ nuclease-free water containing $600 \mathrm{ng}$ insert DNA and $120 \mathrm{ng}$ linearized vector (5:1 ratio). The resulting product was used for two electroporation reactions at $2 \mu \mathrm{l}$ each, thereby creating two distinct small-insert libraries. At electroporation, $2 \mu$ ligation mixture was added to $50 \mu$ l One Shot ${ }^{\circledR}$ TOP10 Electrocomp ${ }^{\mathrm{TM}}$ E. coli cells (Thermo Fisher Scientific). The sample was transferred to a $1 \mathrm{~mm}$ cuvet and subjected to an electric pulse at $1.8 \mathrm{kV}, 2.5 \mu \mathrm{F}$ and $200 \Omega$. To recover the $E$. coli cells, $1 \mathrm{ml}$ SOC medium was added, and the sample was incubated at $37^{\circ} \mathrm{C}$ for $1 \mathrm{~h}$. After recovery, $2 \mu \mathrm{l}$ of the cell suspension was tenfold serially diluted, and plated on LB agar media containing kanamycin $(50 \mu \mathrm{g} / \mathrm{ml})$ in order to estimate library size based on CFU counts. For each library, twelve clones were picked and colony PCRs were performed with primers pZE21_81_104_57C and pZE21_151_174rc_58C [185], which flank the insertion site. The average product size (nt), the CFU count (in no. of colonies per $\mu \mathrm{l}$ ) and the total volume (in $\mu \mathrm{l}$ ) were multiplied in order to estimate the library size. In order to achieve library amplification, the remainder of the cell suspension after recovery was transferred to $10 \mathrm{ml} \mathrm{LB}$ medium with kanamycin $(50 \mu \mathrm{g} / \mathrm{ml})$ and incubated overnight at $37^{\circ} \mathrm{C}$. After library amplification, another CFU count was performed. The total number of CFUs pre- and post-amplification was divided in order to estimate the extent of library expansion. Libraries were mixed to contain $20 \%$ glycerol and stored in cryotubes at $-80{ }^{\circ} \mathrm{C}$. We also verified the function of a $\beta$-lactamase resistance gene by cloning it into the pZE21 vector as a single gene using the methods described in this paragraph, starting from the ligation step. The insert DNA consisted of amplicon sequences that were obtained by gene-specific PCR.

\section{Library screening and insert sequencing}

Libraries were plated at 25X (predicted) library coverage (i.e. every clone is expected to be present 25 times) on LB agar plates containing one of the following antibiotics: ampicillin $(20 \mu \mathrm{g} / \mathrm{ml})$, ciprofloxacin $(1 \mu \mathrm{g} / \mathrm{ml})$, tetracycline $(20 \mu \mathrm{g} / \mathrm{ml})$, chloramphenicol $(20 \mu \mathrm{g} / \mathrm{ml})$, polymyxin B $(2 \mu \mathrm{g} / \mathrm{ml})$, trimethoprim $(2 \mu \mathrm{g} / \mathrm{ml})$, erythromycin $(200 \mu \mathrm{g} / \mathrm{ml})$, cefotaxime $(25 \mu \mathrm{g} / \mathrm{ml})$, rifampicin $(25 \mu \mathrm{g} / \mathrm{ml})$, imipenem $(20 \mu \mathrm{g} / \mathrm{ml})$, gentamicin $(20$ $\mu \mathrm{g} / \mathrm{ml})$, D-cycloserine $(100 \mu \mathrm{g} / \mathrm{ml})$, chlortetracycline $(20 \mu \mathrm{g} / \mathrm{ml})$ and amikacin $(50$ $\mu \mathrm{g} / \mathrm{ml}$ ). E. coli TOP10 cells without a plasmid were used as negative control. Inserts 
from antibiotic resistant clones were Sanger sequenced from both the 5' and 3' flanks with respectively the pZE21_81_104_57C and pZE21_151_174rc_58C primers. Clones were assumed to contain identical inserts if sequences were more than $99 \%$ identical over a stretch of $>400 \mathrm{bp}$. One clone per set of identical clones was selected for further analysis. The full-length inserts of these representative clones were sequenced by primer walking. Sanger sequencing was performed by flanking primers custom-designed with Primer3Plus [281] until GeneStudio version 2.2.0.0 could assemble a contig that spanned the entire insert. Finally, vector sequences were removed by DNA Baser version 3.5.4.2.

\section{Analysis of inserts conferring resistance}

ORFs were identified by MetaGeneMark [282], and the corresponding nucleotide and derived amino acid sequences were extracted. Amino acid sequences were used for BLASTp [283] against the NCBI non-redundant protein database and the CARD database [284]. BLASTn of the sequences against the NCBI nr/nt and CARD databases were performed as well. Resistance functions were also predicted with HMMER (http://hmmer.org/) using the profile hidden Markov models (pHMMs) of the Resfams database as a reference [105]. We defined a resistance gene to be identified with high confidence if it was detected at an E-value of <1E-7 by either BLASTp or BLASTn against the Comprehensive Antibiotic Resistance Database (CARD), or by employing the pHMMs of the Resfams database. In addition, the resistance function needed to match the observed resistance phenotype. InterProScan [285] was used for classifying proteins into families and for predicting the presence of domains. Clustal Omega [286] was used to globally align resistance genes with their best hit in NCBl's non-redundant protein database and to calculate an amino acid identity value. ISfinder [287] was used to identify IS elements. The ACLAME server [288] was used to investigate if parts of the insert sequences were previously identified on mobile genetic elements.

\section{Assignment of inserts conferring resistance to bacterial isolates}

Since one small-insert library was prepared with genomic DNA from 31 different sponge-associated bacterial isolates (as opposed to metagenomic DNA), we could use insert sequence-specific PCRs to assign inserts to the bacterium of origin. For this purpose, primers were designed with Primer3Plus [281]. The reaction mixture of a given detection PCR consisted of: $10 \mu \mathrm{l}$ X Green GoTaq Reaction Buffer 
(Promega, Fitchburg, Wisconsin), $0.2 \mathrm{mM}$ dNTPs (Promega), $1 \mu \mathrm{M}$ forward primer, 1 $\mu \mathrm{M}$ reverse primer, $0.5 \mu \mathrm{l}$ GoTaq $\mathrm{G} 2$ DNA polymerase $(5 \mathrm{U} / \mu \mathrm{l}$, Promega), $1 \mu \mathrm{l}$ genomic DNA (10-20 ng/ $\mu \mathrm{l}$ ), and $32.5 \mu \mathrm{l}$ nuclease-free water (Promega). The PCR program consisted of: initial denaturation of $30 \mathrm{~s}$ at $98{ }^{\circ} \mathrm{C} ; 35$ cycles of denaturation at $98{ }^{\circ} \mathrm{C}$ for $10 \mathrm{~s}$, annealing at $57^{\circ} \mathrm{C}$ for $20 \mathrm{~s}$, and extension at $72{ }^{\circ} \mathrm{C}$ for $20 \mathrm{~s}$; and final extension at $72{ }^{\circ} \mathrm{C}$ for $10 \mathrm{~min}$. The PCR product was analysed on a $1 \%$ agarose gel. If neither the initial PCR nor a repetition with a $1{ }^{\circ} \mathrm{C}$ reduction in annealing temperature yielded a product, then new primers were designed. If an insert sequence-specific PCR yielded a product with genomic DNA from multiple isolates, then the amplification products were sequenced by Sanger sequencing. Inserts were assigned to an isolate if the Sanger sequence was $99-100 \%$ identical to the insert sequence.

\section{Nucleotide sequence accession numbers}

The metagenomic insert sequences were deposited under accession numbers KU577908 to KU577944. The insert that contained blapsV-1 as a single gene was deposited under accession KU926347.

\section{Results}

\section{Composition and screening of metagenomic libraries}

In order to investigate the presence and diversity of functional AR genes in sponge microbiota, small-insert libraries were prepared in E. coli with a mixture of gDNA from 31 bacterial strains isolated from the sponges $A$. aerophoba, $P$. ficiformis, and $C$. candelabrum (library I-31; $0.8 \mathrm{Gbp}$ ), and with DNA directly isolated from the same three sponges (libraries Aa; $0.2 \mathrm{Gbp}, \mathrm{Cc} ; 0.8 \mathrm{Gbp}$, Pf; $1.7 \mathrm{Gbp}$ ). $16 \mathrm{~S}$ ribosomal RNA (rRNA) gene amplicon sequence analysis of the bacterial gDNA pool that was used to prepare library I-31 confirmed that DNA from all 31 isolates was present (data not shown). Amongst these 31 sponge-associated bacteria, resistance to 14 out of 16 antibiotics was observed, whereas merely intermediate resistance, signifying a reduced growth rate, to imipenem was observed. None of the isolates were resistant to rifampicin (Table 4.1). Screening of the four different libraries for AR yielded 37 clones with unique inserts conferring resistance to D-cycloserine $(n=6)$, gentamicin $(n=1)$, amikacin $(n=7)$, trimethoprim $(n=17)$, chloramphenicol $(n=1)$, rifampicin $(n=2)$ and ampicillin $(n=3)$ (Table 4.2). No clones were obtained that were resistant to chlortetracycline, 
polymyxin, erythromycin, ciprofloxacin, cefotaxime, tetracycline or imipenem. Most resistant clones (30 of 37) were derived from library I-31, whereas 2 clones, 0 clones and 5 clones were derived from libraries Aa, Cc and Pf, respectively.

\section{Resistance gene diversity and uniqueness}

The unique full-length inserts (mean insert size $=2,974$ bp $\pm 1,627$ [s.d.]) were sequenced by Sanger sequencing, which occasionally required multiple iterations of primer walking. We defined a resistance gene to be identified with high confidence if the resistance function was assigned at an E-value of <1E-7, and furthermore the resistance function needed to match the observed resistance phenotype.

In total, 26 resistance genes distributed over 24 different inserts were identified that met our confidence threshold (Table S4.1). The uniqueness of the resistance genes was evaluated by performing a global alignment at the amino acid level with the closest BLASTp hit in NCBI's non-redundant protein database. The majority of confidently identified resistance genes obtained from library l-31 (17 of 21) were predicted to code for proteins that had $>70 \%$ identity at the amino acid level with known gene products, whereas proteins encoded by the genes $(n=5)$ obtained from the metagenomic libraries of environmental sponge DNA had $<60 \%$ amino acid identity with known gene products (Figure 4.1A, B). Overall, most confidently identified resistance genes (15/26) were predicted to encode trimethoprimresistance-conferring dihydrofolate reductases (Figure 4.1C). The confidently identified AR genes with the lowest similarity to known genes were predicted to code for a glycerol-3-phosphate acyltransferase and a GNAT family acetyltransferase with respectively $32 \%$ and $36 \%$ amino acid identity with the closest hit in NCBl's nonredundant protein database. Both of these amikacin-resistance-conferring genes were detected in libraries of environmental sponge DNA.

For 13 insert sequences a resistance gene could not be identified meeting our threshold. Those inserts conferred resistance to amikacin $(n=4)$, D-cycloserine $(n=6)$, and trimethoprim $(n=3)$ (Table 4.3, Table S4.2). In these cases, besides BLAST searches against the CARD and NCBI nr/nt databases and motif detection by application of Resfams pHMMs, also protein domain and protein family predications by InterProScan were evaluated in order to manually predict AR gene presence. Most (12 of 13) inserts contained multiple open reading frames (ORFs), which prevented the unequivocal assignment of resistance functions to individual genes. 
For three of four inserts that conferred resistance to amikacin, we predicted the presence of genes encoding aminoglycoside-modifying enzymes. On one of these inserts (clone Env_Ami3) a gene was identified that we predicted to code for an enzyme that putatively modifies amikacin by phosphorylation. On the other two inserts, we predicted genes encoding an aminoglycoside acetyltransferase (clone Iso_Ami2) and an aminoglycoside methyltransferase (clone Iso_Ami3). The fourth insert conferring amikacin resistance (clone Iso_Ami4) contained a single gene that shared $93 \%$ amino acid identity with an amino acid transporter, and as such we predicted the gene to confer AR via antibiotic efflux. Six inserts conferred resistance to D-cycloserine. On all of these six inserts we predicted the presence of genes encoding proteins involved in transmembrane transport based on high similarity to known transporters. We predicted that three of the six D-cycloserine resistanceconferring inserts harboured genes encoding transporters that belong to the major facilitator superfamily. Three other inserts on which an AR gene could not be confidently identified conferred resistance to trimethoprim. On one of these inserts (clone Env_Trim4) ), we predicted that a thymidylate synthethase may be responsible for the resistance phenotype. The two other inserts conferring trimethoprim resistance (clones Iso_trim13 and Iso_trim14) putatively encoded oxidoreductases that could confer trimethoprim resistance. 
둥

일

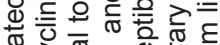

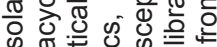

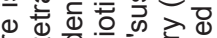

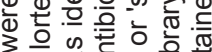

on

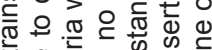

के \&

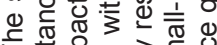

$F$. $\frac{\pi}{0}$.

cis

है

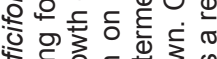

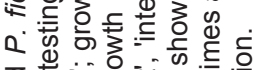

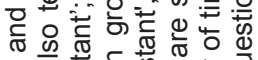

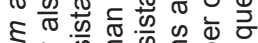

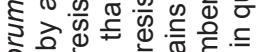

क

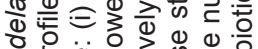

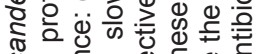

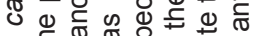

u

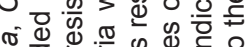

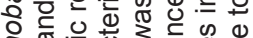



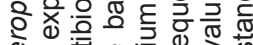

ब

了

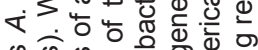

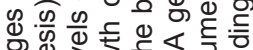

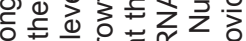

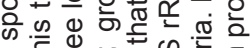

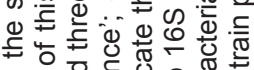

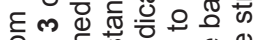

은

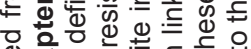

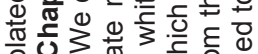

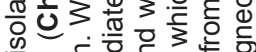

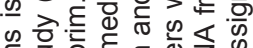

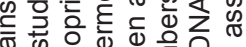

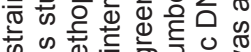
하의

중.윽

造 这

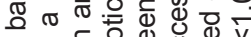

$4 . \subseteq$ 등.응

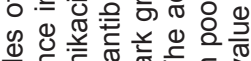

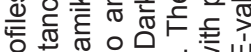

.

응.

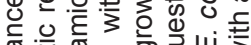

西 음

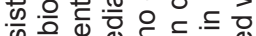

क

称

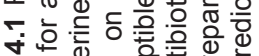

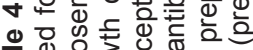

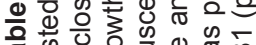

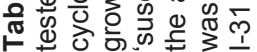

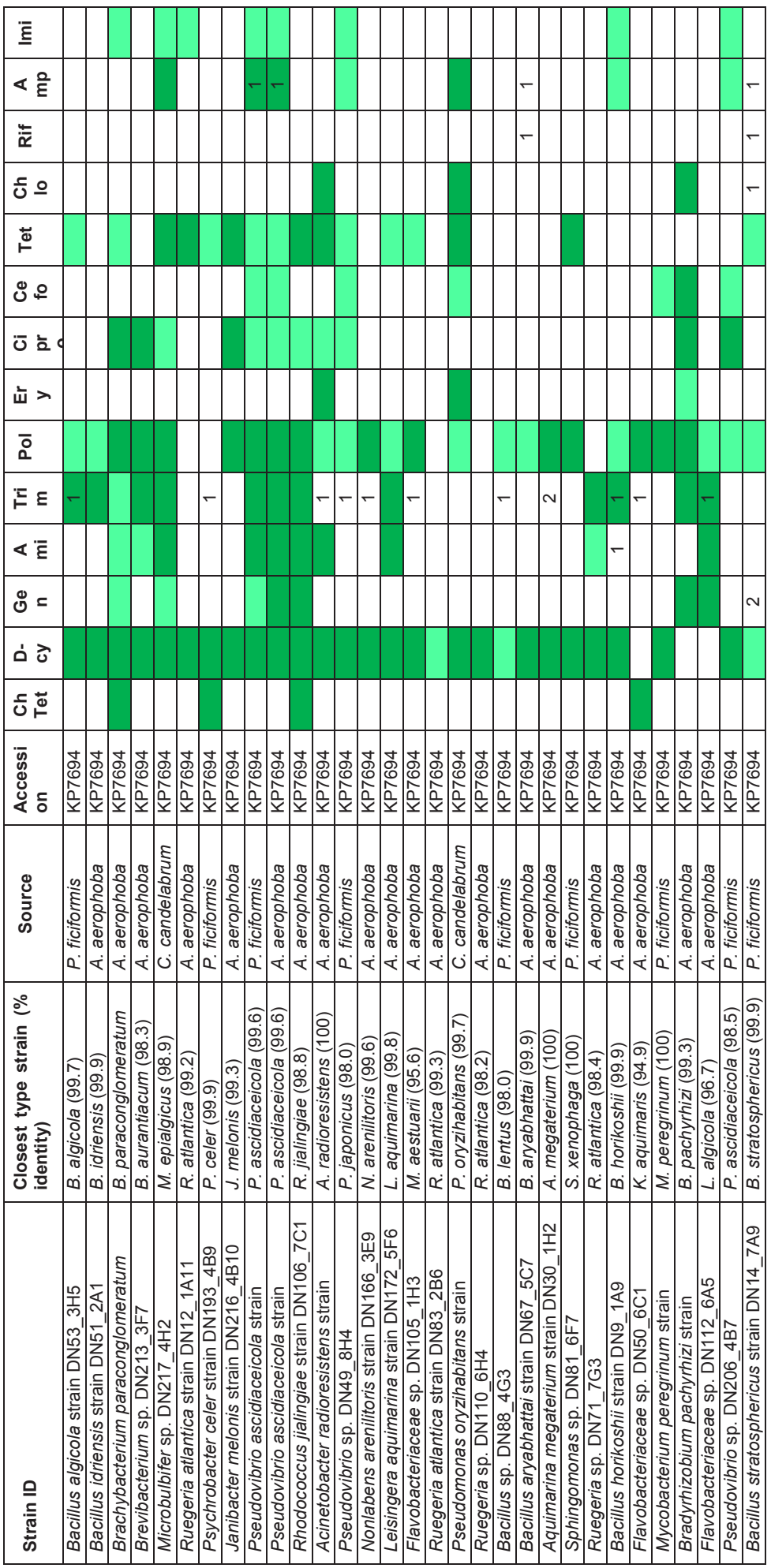


Table 4.2 Small-insert libraries in E. coli were made with DNA isolated from 31 sponge bacteria, and with DNA isolated from A. aerophoba, C. candelabrum and P. ficiformis tissue. The libraries were screened for resistance to 14 antibiotics. This table shows the number of resistant clones with unique inserts for the different metagenomic libraries. No clones were obtained that were resistant to chlortetracycline, polymyxin, linezolid, erythromycin, ciprofloxacine, cefotaxime, tetracycline or imipenem.

\begin{tabular}{|l|c|c|c|c|c|c|c|c|}
\hline Metagenomic library & $\begin{array}{c}\text { Cumulative } \\
\text { library size }\end{array}$ & $\begin{array}{c}\text { D- } \\
\text { cyclose } \\
\text { rine }\end{array}$ & $\begin{array}{c}\text { Gentam } \\
\text { icin }\end{array}$ & $\begin{array}{c}\text { Amikaci } \\
\mathbf{n}\end{array}$ & $\begin{array}{c}\text { Trimeth } \\
\text { oprim }\end{array}$ & $\begin{array}{c}\text { Chlora } \\
\text { mpheni } \\
\text { col }\end{array}$ & $\begin{array}{c}\text { Rifampi } \\
\text { cin }\end{array}$ & $\begin{array}{c}\text { Ampicil } \\
\text { lin }\end{array}$ \\
\hline 31 sponge bacteria (I-31) & $0.8 \mathrm{Gbp}$ & 6 & 1 & 4 & 13 & 1 & 2 & 3 \\
\hline A. aerophoba (Aa) & $0.2 \mathrm{Gbp}$ & 0 & 0 & 1 & 1 & 0 & 0 & 0 \\
\hline C. candelabrum (Cc) & $0.8 \mathrm{Gbp}$ & 0 & 0 & 0 & 0 & 0 & 0 & 0 \\
\hline P. ficiformis (Pf) & $1.7 \mathrm{Gbp}$ & 0 & 0 & 2 & 3 & 0 & 0 & 0 \\
\hline
\end{tabular}

\section{Taxonomic assignment of inserts from the library of $\mathbf{3 1}$ sponge bacteria}

Resistance genes that were confidently identified in library I-31 were assigned to their strain of origin by gene-specific PCRs, where the PCR amplicon covered at least part of the predicted resistance gene. Gene presence was checked in all 31 strains used for building the library by dedicated PCR reactions, which resulted in confidently identified AR genes being assigned to 15 of 31 bacterial strains in library I-31 (Table 4.1). Insert sequences for which no resistance gene could be identified with high confidence were assigned to a strain of origin at the loci given in Table S4.2. We discovered that several inserts may contain hybrid sequences, that is, the inserts consist of two different DNA fragments that were ligated into the vector. For example, the insert of clone Iso_Dcy6 was assigned to Ruegeria spp. in the region between 513-1,372 bp and assigned to Acinetobacter radioresistens DN138_5C8 in the region between 2,240-2,851 bp (Table S4.2). We found that about half of the confidently identified resistance genes (11/21) were assigned to Bacillus spp., and Bacillus was the only taxon to which gentamicin, amikacin, chloramphenicol and rifampicin resistance genes were assigned (Table 4.1, Figure 4.1D). Even though gentamicin, amikacin, chloramphenicol, rifampicin and ampicillin resistance genes were assigned to Bacillus spp., the strains themselves were not found to be resistant to these antibiotics. Resistance genes that were assigned to the remaining taxa comprised mostly trimethoprim resistance genes, with an exception being an ampicillin resistance gene that was assig ned to Pseudovibrio spp. (Table S4.1). Strains to which trimethoprim resistance genes were assigned were not trimethoprim resistant themselves in 8 out of 9 cases. 


\section{Novel $\beta$-lactamase family identified in Pseudovibrio}

The insert sequence of ampicillin-resistant clone Iso_Amp3 contained a gene predicted to encode a class A $\beta$-lactamase. The gene was assigned to Pseudovibrio ascidiaceicola DN64_1D03 and Pseudovibrio ascidiaceicola DN64_8G1, and the predicted protein shared $58 \%$ amino acid identity with the closest hit in the CBMAR database [289]. Since this predicted protein displayed high divergence with members of known $\beta$-lactamase families it is a candidate to be classified into a new family [290]. Therefore, we further tested the gene's function by cloning the gene-specific amplicon sequence that was generated with genomic DNA of Pseudovibrio ascidiaceicola DN64_1D03 as template it into the pZE21 vector. The new clone, PSV1 (accession KU926347), was resistant to ampicillin, which confirmed the functionality of the $\beta$-lactamase gene. Remarkably, the coding sequence of the gene cloned using the genome-derived amplicon sequences was $75 \mathrm{bp}$ longer than the coding sequence of the $\beta$-lactamase gene in the original metagenomic insert. Sanger sequencing of the genome-derived amplicon yielded a gene sequence that shared $100 \%$ nucleotide identity with the gene sequence in clone PSV1. Hence, we concluded that the longer $\beta$-lactamase gene in clone PSV1 is the exact gene that is present in the genome of Pseudovibrio ascidiaceicola DN64_1D03. We predict that even though a frameshift mutation yielded a shorter gene in case of the small-insert library, the encoded enzyme retained its function. We classified the genome-derived (longer) gene into a novel $\beta$-lactamase family named PSV after $\underline{P s e u d o v i b r i o . ~ T h e ~}$ first member was designated blapsv-1. BLASTp of the gene against the CBMAR database showed that LEN and SHV were the closest $\beta$-lactamase families. The best hit from the LEN and SHV families in both cases shared $50 \%$ amino acid identity with blapsv-1. The best hits in the NCBI non-redundant protein sequences database $(\geq 76 \%$ amino acid identity) were exclusively genes in sequenced genomes of Pseudovibrio spp. (Figure 4.2). Additional resistance testing of clone PSV1 showed that blapsV-1 conferred resistance to penicillin $(50 \mu \mathrm{g} / \mathrm{ml})$ but not against cefotaxime $(20 \mu \mathrm{g} / \mathrm{ml})$ and imipenem $(20 \mu \mathrm{g} / \mathrm{ml})$.

\section{Resistance gene dissemination}

Horizontal gene transfer is the main mechanism through which resistance genes are disseminated. Therefore, we investigated our sequences for evidence of gene mobilisation. Comparison of the insert sequences with the ACLAME plasmid 
database showed that they were not previously identified on plasmids. Furthermore, ISfinder did not identify insertion sequences. We investigated whether the resistance genes identified in this study had been observed previously in a different genomic context by comparing our sequences with those in the NCBI nr/nt database (Table S4.3). A $\beta$-lactamase and an ADP-ribosyl transferase (those on clones Iso_Amp2 and Iso_Rif2, respectively), both of which were assigned to Bacillus aryabhattai DN67_5C7, shared very high ( $\geq 99 \%)$ identity with genes previously found in the nonmarine Bacillus megaterium QM B1551, which suggests a relatively recent dissemination event. The insert of clone Iso_Amp2 shared 99\% identity with the genome of Bacillus megaterium QM B1551 over the full-length insert (1,352 bp). This insert produced four other significant alignments with sequences in the NCBI nr/nt database at $>90 \%$ nucleotide identity, all of which belonged to Bacillus megaterium strains. The insert of clone Iso_Rif2 was 6,717 bp in length but only the region from 5,198 to 6,717 bp aligned with the genome of Bacillus megaterium QM B1551. Besides the rifampicin-resistance-conferring gene, this region encodes a hypothetical protein and a gene that putatively encodes an enzyme involved in site specific recombination of plasmids. The gene putatively encoding a recombination enzyme was the only gene involved in gene mobilization that was predicted on the inserts.

\section{Discussion}

Functional metagenomic analysis of sponge-associated bacteria revealed diverse AR genes that conferred resistance to ampicillin, D-cycloserine, gentamicin, amikacin, trimethoprim, chloramphenicol and rifampicin. Seventeen of 26 confidently identified AR genes shared low homology ( $<90 \%$ amino acid identity) with known gene products. Therefore, these results show that sponges, like environments that contain complex bacterial communities, appear to represent reservoirs of unique AR genes. In addition, we obtained 13 inserts that did not contain genes with significant similarity to known resistance genes, reinforcing the notion that sponges may act as reservoirs of yet unknown mechanisms of AR. Two resistance genes shared $\geq 99 \%$ nucleotide identity with AR genes detected outside the marine environment, and hence it is tempting to speculate that AR genes are being transmitted between sponges and non-marine environments and as such may be exploited by bacteria in other non-marine habitats. It has previously been shown that AR genes can be genetically linked, leading to co-selection $[60,291]$. None of our inserts contained AR 


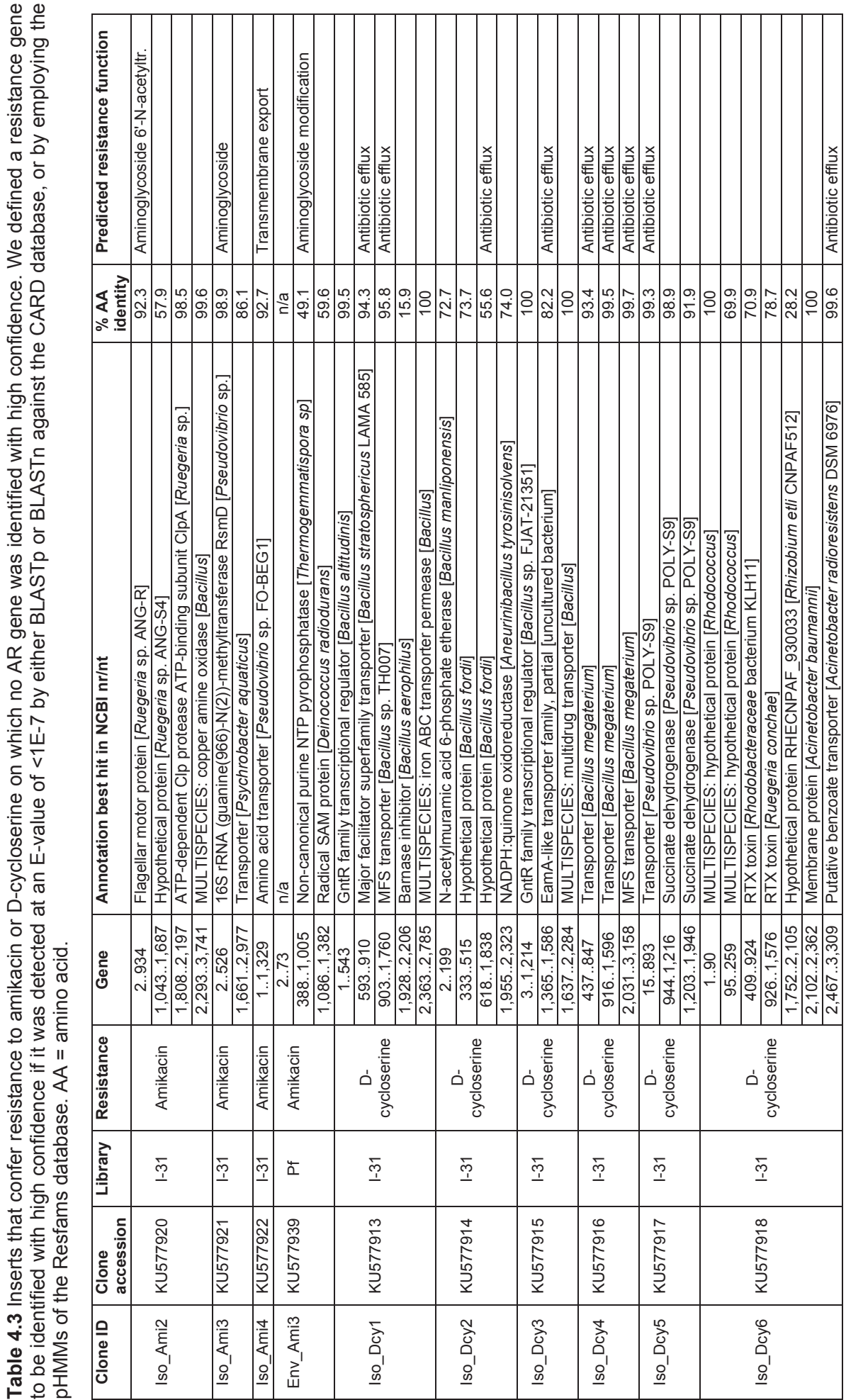


(A)

DNA of 31 bacterial isolates

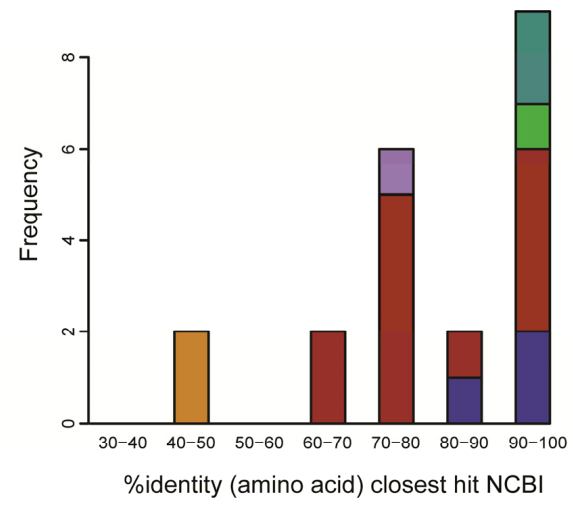

(C)
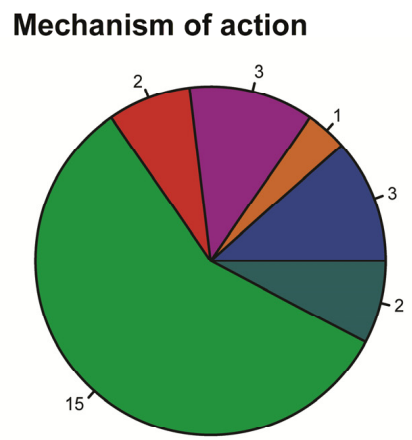

- B-lactamase

- Chloramphenicol acetyltransferase

- Aminoglycoside phosphotransferas

Amınoglycoside acetyltransferase

- Dihydrofolate reductase

- ADP-ribosyl transferase
(B)

\section{Environmental sponge DNA}

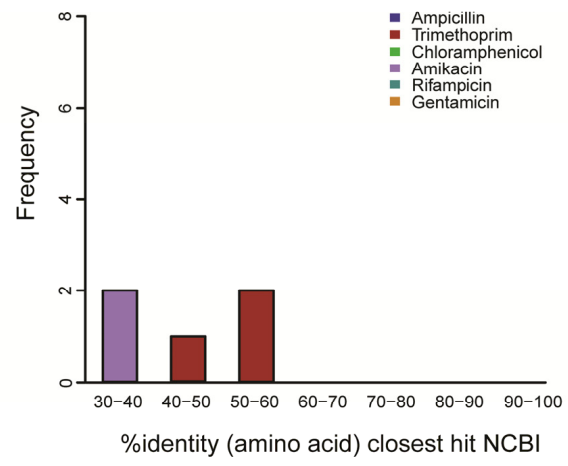

(D)
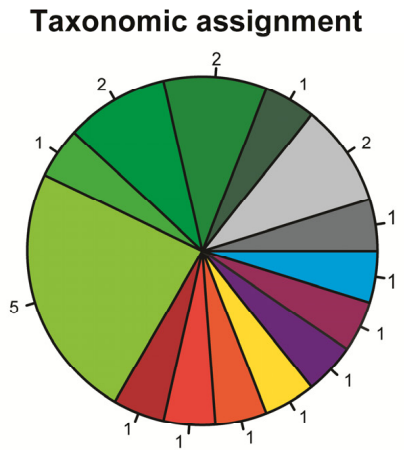

A radioresistens DN138 $5 \mathrm{C} 8$

- Flavobacteriaceae sp. DN105_1H3 A. megaterium $\mathrm{DN} 30-1 \mathrm{H} 2$

B. algicola DN53 $3 \mathrm{H} \overline{5}$

. aryabhattai DN67 $5 \mathrm{C} 7$

B. horikoshii DN9 1A99

Bacillus sp. DN88_4G3
B. stratosphericus DN14 7A9 - Flavobacteriaceae sp. DN112-6A5

N. arenilitoris DN166 3E9

- P. ascidiaceicola DN64_1D03 \& DN64_8G Pseudovibrio sp. DN49_8H4 - P. celer DN193_4B9

Figure 4.1 These illustrations provide information about the 26 resistance genes that were identified with high confidence (E-value $<1.0 \mathrm{E}-7$ ) using the CARD and Resfams databases as a reference. Panels A and B show amino acid identity distributions of these genes with their best hit (bitscore sorted) in NCBI's non-redundant protein database. Panel A applies to resistance genes obtained from the library of 31 sponge bacteria whereas panel B applies to resistance genes obtained from libraries based on DNA isolated from sponge tissue. Panel $\mathbf{C}$ shows the mechanisms of action of all 26 resistance genes. Panel D shows the taxonomy assignments of the resistance genes obtained from the library of 31 sponge bacteria.

genes with different resistance functions, which is likely in part due to the small insert size of the metagenomic libraries.

Out of 37 inserts harbouring resistance genes, 30 inserts were obtained from the library of 31 sponge bacteria, and 7 inserts were obtained from the libraries of sponge tissue even though their cumulative library sizes were $0.8 \mathrm{Gbp}$ and $2.7 \mathrm{Gbp}$, respectively. We expect that this discrepancy can be explained by libraries from sponge tissue DNA containing a substantial fraction of sponge DNA. No identical resistance genes were obtained from individual isolates and from sponge tissue in 


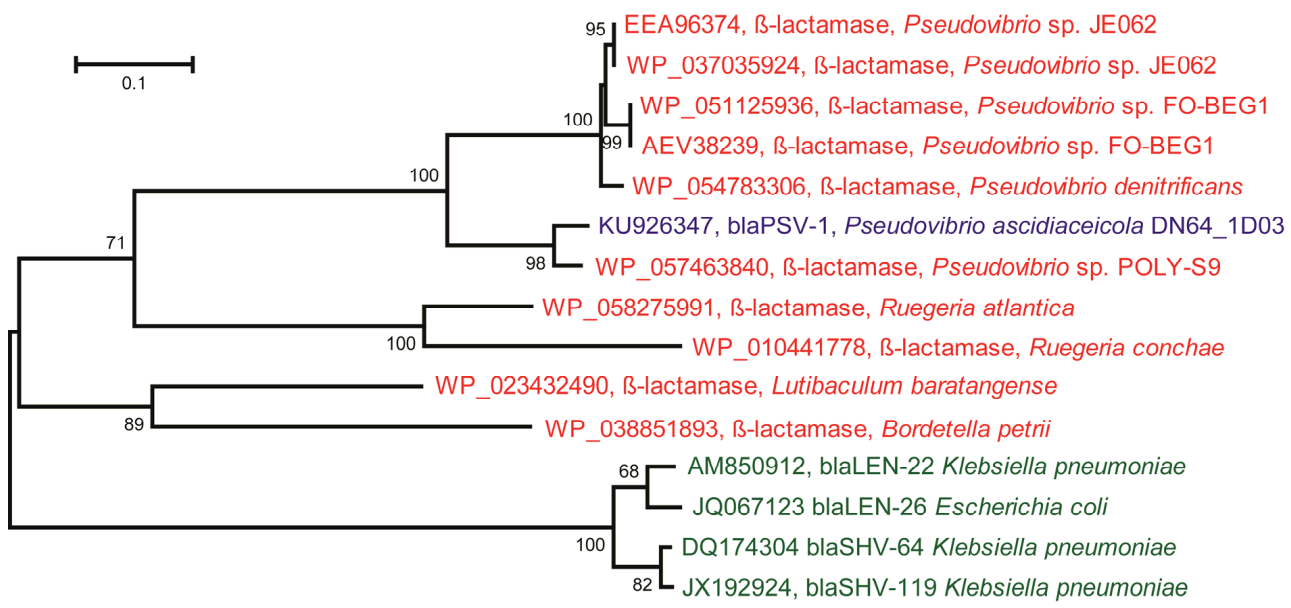

Figure 4.2 Maximum Likelihood Tree based on protein sequences of: the novel ß-lactamase (b/a $\left.a_{\mathrm{PSV}-1}\right)$ discovered in Pseudovibrio ascidiaceicola DN64_1D03 (blue), the closest homologues of the novel ßlactamase in the NCBI non-redundant protein sequences database (red), and the closest homologues of the novel ß-lactamase in the CBMAR database (green) (the two closest homologues were picked for each of the two closest families: $b / a_{\text {LEN }}$ and $\left.b / a_{\text {SHV }}\right)$. In this figure, only the proteins in green and the novel ß-lactamase (blapsv-1) have demonstrated functionality. The tree was constructed in MEGA using 1,000 iterations of bootstrapping. Bootstrap values $<50$ are not shown. The horizontal bar indicates the number of amino acid substitutions per site.

spite of the individual isolates being previously isolated from the tissue samples. This result, and taking into account the high AR gene diversity and limited scale of the experiment, suggests the presence of a substantial number of AR genes in sponges that are not yet identified. The number of AR genes that we identified was also restricted to those that are compatible with the expression system of the $E$. coli host, and to those that confer AR based on a DNA sequence of at most $5 \mathrm{kbp}$. From the sponge $C$. candelabrum no resistant clones were obtained, neither from the sponge tissue nor from the individual isolates. However, it should be noted that the smallinsert library of 31 bacteria contained only two isolates from $C$. candelabrum, which reduced the chance to identify a resistance gene from this sponge. Furthermore, the library of C. candelabrum tissue was $0.8 \mathrm{Gbp}$ in size and as such is expected to cover only a small fraction of the total unique DNA in this niche. Therefore, absence of resistance inserts for this sponge species should not be interpreted as an absence of AR genes.

Most confidently identified resistance genes (15/26) conferred resistance to trimethoprim by encoding the enzyme dihydrofolate reductase. Dihydrofolate reductase is a housekeeping enzyme that converts dihydrofolic acid to tetrahydrofolic acid. Tetrahydrofolic acid in turn is a required cofactor to convert dUMP to dTMP by 
thymidylate synthase, with dTMP being essential for DNA synthesis. Trimethoprim resistance due to additional dihydrofolate reductase genes can occur via (a combination of) two mechanisms: (i) the dihydrofolate reductase is overproduced leading to higher levels of the enzyme in the cell, and (ii) the heterologously introduced dihydrofolate reductase has decreased trimethoprim susceptibility due to mutations [65]. The fact that strains to which dihydrofolate reductase genes were assigned were themselves in most cases (8/9) not trimethoprim resistant, may have resulted from these strains having too little active dihydrofolate reductase to compensate for the inhibition by trimethoprim. Resistance in recombinant $E$. coli clones that host a plasmid-encoded dihydrofolate reductase may result from $50-70$ copies of pZE21 plasmid being present per cell [292], thereby substantially increasing the concentration of active enzyme. Expression level differences of active enzyme could also explain other instances where the host to which an AR gene was assigned was itself not resistant. For example, Bacillus stratosphericus DN14_7A9 contained functional genes conferring resistance to gentamicin, chloramphenicol, rifampicin and ampicillin, but the strain itself was not resistant to these antibiotics. In the natural environment, these AR genes can potentially benefit their bacterial hosts in the presence of lower levels of antimicrobials than those applied in this study, or even if antimicrobials are present at sub-MIC levels [293, 294]. For example, an E. coli strain harbouring a single GyrA mutation was shown to outcompete the wild-type strain at a ciprofloxacin level of $1 / 230$ the wild-type MIC [216].

The most unique confidently identified (predicted) resistance genes were those encoding a glycerol-3-phosphate acyltransferase and a GNAT family acetyltransferase with respectively $32 \%$ and $36 \%$ amino acid identity with the closest hit in NCBl's non-redundant protein database. These genes were derived from libraries $\mathrm{Aa}$ and $\mathrm{Pf}$, respectively. Although aminoglycoside resistance genes might serve as defense against locally or self-produced antimicrobials [295], past studies have ascribed to these genes various other functional roles besides aminoglycoside modification. Aminoglycoside modification would then be accidental. For instance, a 2'-N-acetyltransferase was shown to be involved in the acetylation of peptidoglycan [196]. Reeves et al. [210] suggested roles for aminoglycoside resistance genes in immune modulation and alleviation of cellular stress. In addition, aminoglycoside-3'phosphotransferases are closely related to protein kinases [296]. For one amikacinresistance conferring insert, we predicted that resistance resulted from antibiotic 
efflux. The efflux pump showed high identity with an amino acid transporter that was not previously implicated in amikacin resistance. For another six metagenomic inserts from our libraries that conferred D-cycloserine resistance, we predicted the presence of efflux pumps that were also not previously linked to resistance. These results show that sponge-derived bacteria carry genes that provide AR by mechanisms that were not previously observed.

One novel $\beta$-lactamase (blapsv-1) was discovered in $P$. ascidiaceicola DN64_1D03 that provided resistance to ampicillin and penicillin. Based on high amino acid sequence divergence with known $\beta$-lactamase families, the gene was

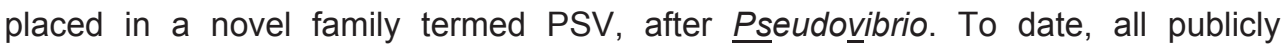
available sequences that share $\geq 76 \%$ identity with blapsv-1 at the amino acid level were found in Pseudovibrio spp. (Figure 4.2), a genus whose members are consistently being isolated from sponges and have never been isolated outside the marine environment [146-148]. The subsequent closest homologues of the novel $\beta$ lactamase were (predicted) $\beta$-lactamases from Ruegeria, a genus comprising marine bacteria [297]. Therefore, blapsv-1 appears to be a marine-specific $\beta$-lactamase, and may correspondingly have a marine-specific role and/or substrate. In sponges, $\beta$ lactamases could serve as a defense molecule against $\beta$-lactam antimicrobials, and substances containing a $\beta$-lactam ring have been identified in sponges [298]. On the other hand, $\beta$-lactamases have also been predicted to serve a role in disruption of cell signalling [299]. Apart from the biological role of $\beta$-lactamases, their evolutionary origin is also still unclear [300, 301].

The majority of confidently identified AR genes (11/21) in library I-31 were assigned to Bacillus spp. including AR genes conferring resistance to ampicillin, chloramphenicol, gentamicin, amikacin and rifampicin. This is in line with the fact that, to date, the only sponge isolate that was shown to harbour functional resistance genes was Bacillus sp. strain HS24 [139, 140]. These findings suggest that in sponges, Bacillus spp. are a reservoir of AR genes. However, sponges filter thousands of litres of water per day [302], and as a result, sponge-associated microorganisms are not always permanent residents of the sponge holobiont. In fact, in all three investigated sponges Bacillus spp. represent less than $0.1 \%$ of the sponge microbiome (Chapter 3 of this thesis). Therefore, we speculate that the diverse AR genes detected in the sponge-associated Bacillus spp. do not play a 
sponge-specific role but rather that these genes serve a more general role in the survival of these bacteria in the marine environment.

A phenomenon we noticed is that in our study, several inserts may contain hybrid sequences, that is, the inserts consist of two different DNA fragments that were ligated into the same vector. As a consequence, the assignment of inserts to a strain of origin can depend on the sequence locus that is amplified by insert sequence-specific PCR. We suspect that these hybrid inserts are an artefact of the cloning strategy involving ligation of blunt end fragments. Because in the present study, one of the libraries was prepared from DNA extracted from 31 bacterial isolates, and thus a defined pool of starting DNA was used, detection of hybrid inserts was possible. However, based on this finding, for all studies using similar cloning methods conclusions about the genetic context of AR genes on metagenomic inserts should be done carefully. Here, to ensure that all confidently identified resistance genes were assigned to the correct strain, we performed gene-specific PCRs (Table S4.1).

In conclusion, we show that sponges are a reservoir of diverse functional AR genes. We detected functional AR genes that show little similarity to known AR genes, as well as functional AR genes that are completely novel. One ampicillin resistance gene, blapsv-1, was placed into a novel family of marine-specific $\beta$ lactamases. These results raise questions as to the roles of these genes in bacteria residing in arguably the oldest lineage of metazoans [124], the sponges. Furthermore, the functionality of our observed AR genes in E. coli shows that they can potentially be harnessed by phylogenetically distinct bacteria in other environments.

\section{Acknowledgements}

This work was supported by the European Union through the EvoTAR project (Grant agreement no. 282004) and by a grant of BE-Basic-FES funds from the Dutch Ministry of Economic Affairs (project 7.1.5). MOAS and MRE additionally acknowledge the Lundbeck Foundation for financial support. 


\section{Supplementary information}

\section{Supplementary Tables and Figures}

Tables S4.1, S4.2 and S4.3 are available upon request. 



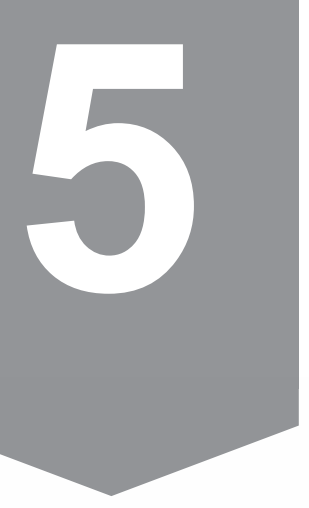

Comparative genomics of the marine genus Pseudovibrio highlights symbiotic capacities of versatile bacteria 


\section{Abstract}

Pseudovibrio is a marine bacterial genus, members of which are most often isolated from sessile marine animals, and sponges in particular. Even though the metabolic versatility of Pseudovibrio spp. allows them to thrive in diverse environments, it is hypothesised that they preferentially form mutualistic relationships with their hosts. Here, we studied evolutionary and symbiotic relations between Pseudovibrio and their eukaryotic hosts by comparative genomics of 31 Pseudovibrio strains of which 25 were isolated from sponges. All strains were highly similar in terms of core metabolic pathways, albeit with substantial differences in overall gene content. Based on gene composition Pseudovibrio spp. clustered by geographic region (i.e. Caribbean, Mediterranean, Atlantic and Pacific), indicating geographic speciation. Furthermore, the fact that isolates from the Mediterranean Sea clustered by sponge (i.e. Aplysina aerophoba, Corticium candelabrum and Petrosia ficiformis) suggested host-specific adaptation or colonization. Based on gene content, Pseudovibrio hongkongensis is only distantly related to the other Pseudovibrio spp. and should not be classified as Pseudovibrio. All Pseudovibrio genomes were found to encode a large number of proteins with SEL1 and tetratricopeptide repeats, which have been suggested to play a role in host colonization. Our analysis showed that for evasion of the host immune system, Pseudovibrio spp. may depend on type III, type IV and type VI secretion systems that can inject effector molecules directly into eukaryotic cells. In addition, the detection of on average seven secondary metabolite biosynthesis clusters per genome reinforced the role of Pseudovibrio spp. as potential producers of novel bioactive compounds. Tropodithietic acid, bacteriocin and terpene biosynthesis clusters were highly conserved within the genus, suggesting an essential role in survival e.g. through growth inhibition of bacterial competitors. Taken together, these results support the hypothesis that Pseudovibrio spp. form mutualistic relationships with sponges. 


\section{Introduction}

Pseudovibrio is a genus of Gram-negative, heterotrophic, facultative anaerobic, marine $\alpha$-Proteobacteria. The genus was proposed by Shieh and collegues [143], and presently six species have been described, including $P$. denitrificans [143], $P$. ascidiaceicola [152], P. japonicus [157], P. axinellae [303], P. hongkongensis [144] and $P$. stylochi [145]. To date, members have been isolated from sea water [157], ascidians [152], a flatworm [145], tunicates [153, 154], corals [155] and sponges [272]. Members of the genus Pseudovibrio are characterized as motile, rod-shaped halophilic bacteria. For energy production, they are capable of $\mathrm{O}_{2}$-dependent respiration as well as fermentation and/or $\mathrm{NO}_{3}{ }^{-}$- and $\mathrm{NO}_{2}{ }^{-}$-dependent (anaerobic) respiration. The study of two Pseudovibrio genomes showed that they are metabolically versatile, i.e. they are capable of utilising a wide range of organic and inorganic compounds to meet their carbon, nitrogen, phosphorous and energy requirements [151].

The relationship between Pseudovibrio and sponges is particularly interesting because they have consistently been isolated from different sponge species [146148], while never simultaneously being isolated from nor detected in surrounding sea water [149, 150]. Although it should be noted that the relative abundance of Pseudovibrio spp. in the sponge microbiota is generally low considering that their presence is rarely detected by cultivation-independent assays [150], these findings indicate that Pseudovibrio spp. are enriched in sponges, reinforcing the current hypothesis that they are symbionts of sponges. Further support for this hypothesis comes from the fact that Pseudovibrio spp. were reported to be vertically transmitted through sponge larvae of Mycale laxissima [150]. Furthermore, Pseudovibrio spp. were suggested to be involved in sponge health as they constituted a lower relative abundance in the culturable community of diseased specimen of Rhopaloeides odorabile [149, 164]. In addition, members of Pseudovibrio were reduced in relative abundance in diseased individuals of the sponge Callyspongia aff. biru [165].

The symbiotic role of Pseudovibrio in the sponge host is still unclear but it could include functions in nutrient uptake [149], denitrification [143, 162, 163], or host defense [161, 166]. In addition, genomic data indicated several mechanisms for establishing and maintaining symbiosis e.g. by interactions of Pseudovibrio with the host immune system or cytoskeleton [151, 304, 305]. Pseudovibrio spp. have 
received particular interest as sources of clinically relevant antimicrobials [158, 159], with multiple studies reporting that the genus is highly bioactive [166, 306]. So far, Pseudovibrio-derived secondary metabolites that have been identified are phenazine [160], tropodithietic acid (TDA) [161, 307] and pseudovibrocin [156]. Pathways of polyketide synthases (PKS) and non-ribosomal peptide synthethases (NRPS), and/or their hybrids are particularly important in the production of secondary metabolites by Pseudovibrio spp. [159, 308, 309], albeit not for TDA synthesis.

In this study, we compared the genomes of 31 Pseudovibrio isolates, including 25 sponge isolates and five type strains, implementing the Semantic Annotation Pipeline for Prokaryotes [310]. We resolved phylogeny, and studied metabolic pathways and secondary metabolite biosynthesis (SMB). In addition, we predicted features of antibiotic resistance and host-symbiont interactions, providing new insight in the nature of the association between Pseudovibrio spp. and marine sponges.

\section{Materials and methods}

\section{Sample collection and data deposition of all analysed genomes}

The type strains $P$. ascidiaceicola DSM $16392^{\top}, P$. axinellae DSM $24994^{\top}, P$. denitrificans $\mathrm{JCM} 12308^{\top}$ and $P$. japonicus $\mathrm{NCIMB} 14279^{\top}$ were obtained from the respective culture collections. Sponge-associated Pseudovibrio strains were isolated from the sponges $C$. candelabrum $(n=2), P$. ficiformis $(n=10)$ and $A$. aerophoba $(n=7)$ as described in Chapter 3 of this thesis. Pseudovibrio sp. AB108, Pseudovibrio sp. AB111, Pseudovibrio sp. AB113 and Pseudovibrio sp. BC118 were isolated from respectively an Ircinia sp., Chondrilla nucula, Acanthella acuta and Cellepora pumicosa, in the Limski Channel in Croatia $\left(45^{\circ} 7^{\prime} 54.56^{\prime \prime} \mathrm{N}, 13^{\circ} 39^{\prime} 13.02^{\prime \prime} \mathrm{E}\right)$. The type strain $P$. hongkongensis UST20140214-015B ${ }^{\top}$ was genome sequenced but not included in growth experiments. Publicly available genome sequences of Pseudovibrio FO-BEG1 (GenBank accession numbers: CP003147 and CP003148), Pseudovibrio JE062 (ABXL00000000), Pseudovibrio POLY-S9 (LCWX00000000), Stappia stellulata DSM 5886 ${ }^{\top}$ (AUIM00000000), Nesiotobacter exalbescens LA33B $^{\top}$ (AUGS00000000), and Labrenzia alexandrii DFL-11 ${ }^{\top}$ (ACCU00000000) were downloaded and re-annotated applying the methods particular to this study.

\section{DNA isolation and sequencing}

The Pseudovibrio strains were inoculated from glycerol stocks onto marine agar 2216 (Difco, Detroit, United States). Subsequently, a single colony was grown at $20^{\circ} \mathrm{C}$ in 
marine broth 2216 (Difco), and DNA was isolated using the MasterPure ${ }^{\text {TM }}$ DNA Purification Kit (Epicentre, Madison, United States). Genome sequencing was done using the Illumina MiSEQ (paired end, 2 x 300 bp reads) at GATC Biotech (Konstanz, Germany). The genome of type strain $P$. hongkongensis UST20140214-015B ${ }^{\top}$ was sequenced on the Illumina Hiseq 2000 (paired end, 2 x 101 bp reads).

\section{Antibiotic resistance}

Resistance profiles were determined by inoculation of pre-grown liquid cultures onto marine agar containing one of the following (combination of) antibiotics: $20 \mu \mathrm{g} / \mathrm{ml}$ polymyxin B, $20 \mu \mathrm{g} / \mathrm{ml}$ daptomycin, $50 \mu \mathrm{g} / \mathrm{ml}$ vancomycin, $50 \mu \mathrm{g} / \mathrm{ml}$ penicillin, 20 $\mu \mathrm{g} / \mathrm{ml}$ erythromycin, $20 \mu \mathrm{g} / \mathrm{ml}$ ciprofloxacin, $100 \mu \mathrm{g} / \mathrm{ml}$ cefotaxime, $50 \mu \mathrm{g} / \mathrm{ml}$ tetracycline, $50 \mu \mathrm{g} / \mathrm{ml}$ chloramphenicol, $95 \mu \mathrm{g} / \mathrm{ml}$ sulfamethoxazole and $5 \mu \mathrm{g} / \mathrm{ml}$ trimethoprim, $20 \mu \mathrm{g} / \mathrm{ml}$ lincomycin, $100 \mu \mathrm{g} / \mathrm{ml}$ kanamycin, $20 \mu \mathrm{g} / \mathrm{ml}$ rifampicin, $20 \mu \mathrm{g} /$ $\mathrm{ml}$ linezolid, $50 \mu \mathrm{g} / \mathrm{ml}$ ampicillin or $20 \mu \mathrm{g} / \mathrm{ml}$ imipenem. Antibiotic resistance was evaluated two days post-inoculation and compared to growth on media without antibiotics as a reference. We defined three levels of antibiotic resistance: (i) 'resistant'; growth of the bacteria was identical to their growth on media without antibiotics, (ii) 'intermediately resistant'; growth of the bacteria was slower than growth on media without antibiotics, and (iii) 'susceptible'; no growth.

\section{Genome assembly and quality control}

The read quality and presence of Illumina Truseq adapter sequences was investigated with FASTQC [311], and Illumina Truseq adapter sequences were removed with Cutadapt [312]. All genomes were assembled with the A5-miseq assembler [313] using default settings with as exception the $P$. hongkongensis UST20140214-015B ${ }^{\top}$ genome that was assembled by Velvet 1.2.10 [314]. Subsequently, Pilon 1.13 [315] was used to improve the assemblies based on the assembled scaffolds and error-corrected reads obtained as output from the A5 assembler. Assemblies were investigated for contamination by BLASTn [283] using the $\mathrm{NCBI} \mathrm{nr} / \mathrm{nt}$ database as a reference. Overall, seven $<3 \mathrm{~kb}$ contigs were removed, which were assigned to Clostridia and Bacilli spp. Sequences from Enterobacteria phage phiX174 were removed as well as DNA of this phage is routinely included as a positive control during sequencing. In order to determine the coverage, A5 errorcorrected reads were aligned with the assemblies by Bowtie2 2.2 .5 [316] using default settings. The resulting SAM file was converted by Samtools 0.1.19 [317] into 
a BAM file, after which the coverage per base was calculated using Bedtools 2.25.0 [318].

\section{Genome annotation}

The genomes of all Pseudovibrio strains were (re-)annotated. In addition, the genomes of Labrenzia alexandrii DFL-11 ${ }^{\top}$ [319], Stappia stellulata DSM $5886^{\top}$ and Nesiotobacter exalbescens DSM $16456^{\top}$ were annotated in order to serve as a reference. A local installation of Galaxy [320] was used to execute the tools for annotation. FASTA files containing the genomic scaffolds were converted to RDF format to detect rRNA genes by RNAmmer [321], and to detect tRNA and tmRNA genes by ARAGORN [321]. Prodigal [322] was used to predict genes, and subsequently the InterPro Database 5.4-47.0 [285] was used to classify the genes and to predict domains and sites. At runtime, the following databases were selected: TIGRFAM, ProDom, SMART, PROSITE, PfamA, PRINTS, SUPERFAMILY, Coils and Gene3d. PRIAM [323], SignalP 4.0 [324], and TMHMM [325] were used to respectively detect ECs, signal peptides, and transmembrane protein topology. Finally, output from the different tools was merged into a single RDF file for subsequent analyses.

\section{Phylogeny, functional diversity and environmental distribution}

A phylogenetic tree was made in ARB with the 16S rRNA gene sequences from the genomes. When multiple 16S rRNA genes were found in a genome that were $>99 \%$ identical, a single gene was picked to be included in the tree. The three closest nonPseudovibrio relatives in the Silva guide tree (release 115) from which the genomes were publicly available were used as outgroup. Gene alignments were manually curated, and a Maximum Likelihood tree was constructed with 1,000 iterations of rapid bootstrapping. Average nucleotide identity between the different genomes based on BLAST results (ANIb) was calculated using JSpecies v1.2.1 with default parameters [326]. The dissimilarity in terms of protein diversity was analysed and visualized in a distance tree. To identify homologous proteins shared between the genomes, an all-against-all BLASTp was performed, after which OrthAgogue [327], and MCL [328] were used to identify orthologous groups of proteins (OGPs). With R version 3.2.2. [329], a matrix with the binary distances was created based on the presence/absence of the OGPs that are present in $>2$ genomes. The distance matrix was used for complete linkage hierarchical clustering. To assess the uncertainty in 
the hierarchical cluster analysis we used the R package PVC with 10,000 bootstraps. Canoco 5 [254] was used to investigate which environmental variables could explain the variation in OGPs composition amongst the genomes. The presence of metabolic pathways was predicted using OGPs that had EC numbers assigned, and using the MetaCyc database [330] as a reference. Presence/absence data of OGPs that are present in at least three genomes was used as input for SIMPER [255] in order to break down the contribution of each OGP to the observed dissimilarity between genomes grouped by environmental variables. DIAMOND BLAST v.0.7.12 [331] was used to assign all protein sequences to Clusters of Orthologous Groups (COGs) with the 2014 edition of the COGs database as a reference [332]. The environmental distribution of Pseudovibrio spp. was investigated using the IMNGS platform (www.imngs.org). Full-length 16S rRNA gene sequences of the type strains were used as query and the similarity threshold was set at $99 \%$ nucleotide identity.

\section{Annotation of symbiosis-related genes}

Interproscan [285, 333] was used to detect the following potentially symbiosis-related gene products: ankyrin repeat proteins (IPR020683 and IPR002110), proteins with SEL1 repeats (IPR006597), tetratricopeptide repeat proteins (IPR011990, IPR019734, IPR013105, IPR001440 and IPR011717), Invasion protein B (IPR010642), proteins with the TadE-like domain (IPR012495), LuxI (IPR016181) and LuxR (IPR020642 and IPR012495). Proteins with SEL1 repeats and tetratricopeptide repeats are occasionally wrongly classified due to their high similarity. Therefore, we used TPRpred [334] to correctly categorize proteins with these repeats using an E-value of $1.0 \mathrm{E}^{-7}$ as cut-off. The presence of Type III secretion systems (T3SSs), Type IV secretion systems (T4SSs) and Type VI secretion systems (T6SSs) was investigated in the genomes with MultiGeneBlast [335] using default settings with as query previously identified gene clusters that were described by [305] i.e. FO-BEG1_3657-03696 (T3SS), PsW64_00918-00938 (T4SS), FO-BEG1_01844-01855 (T6SS-I) and FO-BEG1_02827-02846 (T6SS-II).

\section{Detection of antibiotic resistance genes and SMB clusters}

To predict the capacity for TDA biosynthesis, the presence of $t d a A B C D E F$ and $t d a H$ genes was assessed. Therefore, BLASTp using the sequences of UniProtKB accessions I7F4K4_PHAIB (tdaA), I7E6B2_PHAIB (tdaB), I7ESQ7_PHAIB (tdaC), I7F2B2_PHAIB (tdaD), I7DWF3_PHAIB (tdaE), I7ESP4_PHAIB (tdaF) and 
G8PGC0_PSEUV (tdaH) was performed against the genome-encoded proteins. Furthermore, the presence of the genes paal, paaJ, paaK, cysl and ma/Y was analysed by investigating if genome-encoded proteins were assigned the EC numbers 3.1.2. (paal), 2.3.1.174 (paaJ), 6.2.1.30 (paaK), 1.8.1.2 (cysl) and 4.4.1.8. (MalY). AntiSMASH version 3.0.4 was used to identify SMB clusters [336]. Antibiotic resistance functions were predicted with HMMER (http://hmmer.org/) using the pHMMs of the Resfams database [105]. In addition, BLASTp of amino acid sequences against the CARD database [284] was performed.

\section{Results and discussion}

\section{Metadata and genome characteristics}

The genomes of 31 Pseudovibrio strains were analysed (Table 5.1), which included five type strains that are currently characterized within the genus. The genomes of 28 Pseudovibrio strains were sequenced as part of this study whereas the genomes of strains Pseudovibrio sp. FO-BEG1, Pseudovibrio sp. JE062 and Pseudovibrio sp. POLY-S9 were obtained from previous studies [151, 304]. Out of the 31 Pseudovibrio strains, two were isolated from sea water, 25 were isolated from sponges, and four were isolated from other sessile marine animals. The genome sizes of all 31 Pseudovibrio strains, except for $P$. hongkongensis UST20140214-015B ${ }^{\top}$, were large (6 $\mathrm{Mb} \pm 0.6$ [s.d.]. The genome of $P$. hongkongensis was only $3.75 \mathrm{Mb}$ and correspondingly, the genome also contained fewer unique protein domains. The GCcontent of the Pseudovibrio strains ranged between $44.6 \%$ for Pseudovibrio sp. AB108 and $52.4 \%$ for Pseudovibrio sp. FO-BEG1, whereas the GC-content of the three close relatives were $63.9 \%$ for Stappia stellulata DSM $5886^{\top}$ isolated from marine sediment [337], 54.6\% for Nesiotobacter exalbescens LA33B $^{\top}$ isolated from water in a hypersaline lake [338], and $57.4 \%$ for Labrenzia alexandrii DFL-11 ${ }^{\top}$ isolated from a culture of the dinoflagellate Alexandrium lusitanicum [319]. 


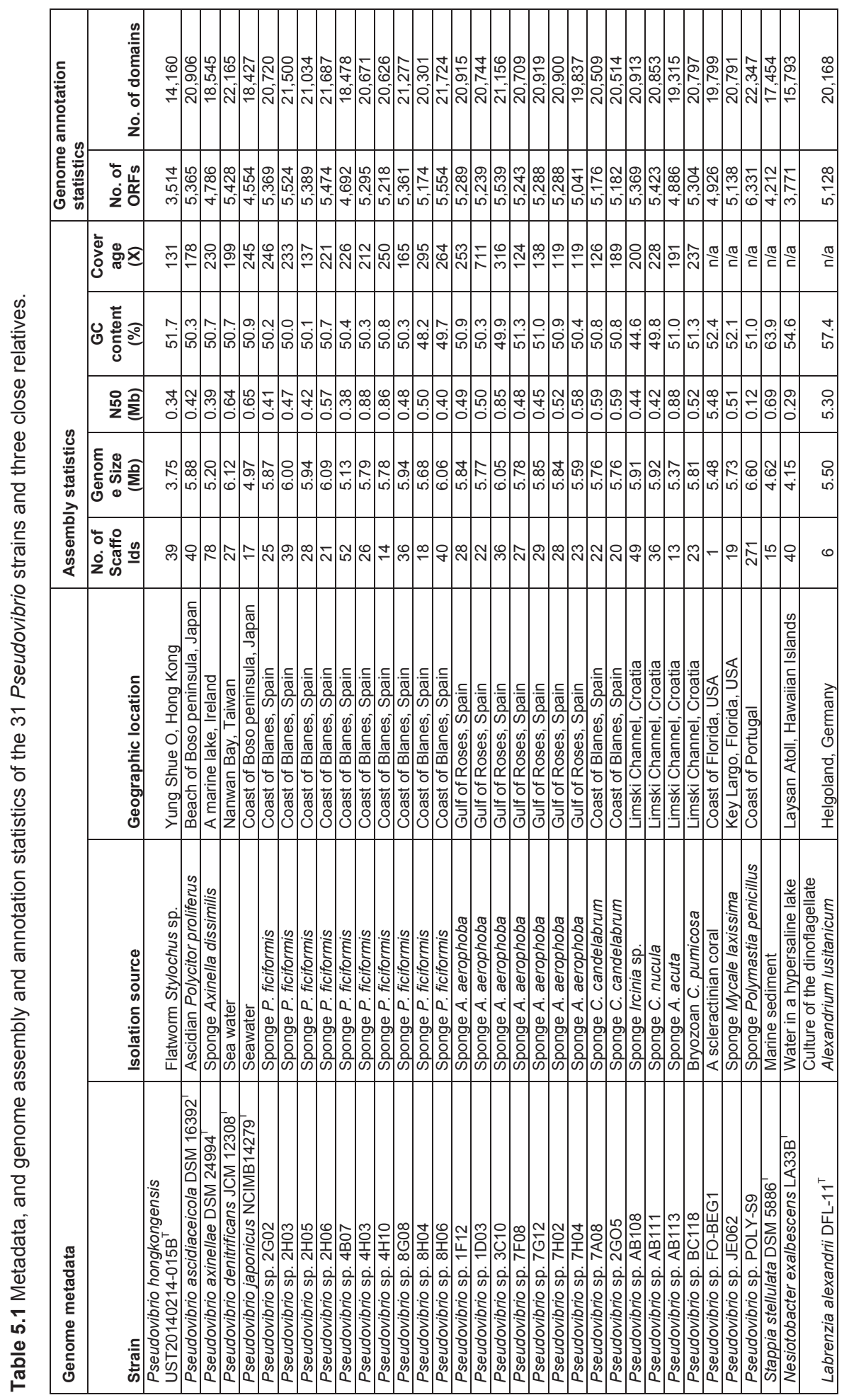




\section{Phylogeny and taxonomy}

Phylogeny and diversity of the strains was assessed based on 16S rRNA gene sequences retrieved from the respective genomes as well as the presence of orthologous groups of proteins (OGPs). Phylogenetic analysis based on 16S rRNA gene sequences placed all Pseudovibrio type strains in separate branches of the corresponding tree (Figure 5.1). Most Pseudovibrio strains (23 of 31) could be classified at the species level to $P$. ascidiaceicola based on the phylogenetic tree and 16S rRNA gene identity values (Table S5.1). Further phylogenomic analysis of these strains based on the presence of OGPs placed $P$. hongkongensis UST20140214$015 \mathrm{~B}^{\top}$ closer to the non-Pseudovibrio outgroup members as compared to the $16 \mathrm{~S}$ rRNA gene based phylogenetic analysis (Figure 5.2). In addition, the average nucleotide identity (ANI) between the $P$. hongkongensis type strain and all other Pseudovibrio spp. strains was $<75 \%$, which was comparable to ANI values observed for the three outgroup species that all belong to different genera (Table S5.2). As such, the data presented here suggest that $P$. hongkongensis UST20140214-015B ${ }^{\top}$ should not be considered a typical member of the Pseudovibrio genus and therefore might need to be placed in a separate genus. For this reason, it was treated as an outgroup member in all subsequent analyses. Furthermore, Pseudovibrio sp. strain $8 \mathrm{H} 04$ and Pseudovibrio sp. strain 4B07 were found in isolated branches in both trees (Figure 5.2), and 16S rRNA gene identity values did not allow species classification because both these strains shared between $97.6 \%$ and $98.6 \%$ with the four most closely related type strains: Pseudovibrio ascidiaceicola DSM $16392^{\top}$, Pseudovibrio denitrificans JCM $12308^{\top}$, Pseudovibrio japonicus $\mathrm{NCIMB} 14279^{\top}$ and Pseudovibrio axinellae DSM $24994^{\top}$. This result, and the fact that the ANI values between these two strains and the Pseudovibrio spp. type strains are rather divergent (Pseudovibrio strain 4B07 and strain $8 \mathrm{H} 04$ share respectively $94.3 \%$ and $97.1 \% \mathrm{ANI}$ with the most similar type strain), indicates that they likely represent two distinct novel species within the Pseudovibrio genus [326]. These strains would not have been recognized as a novel species based on 16S rRNA gene similarity only, and hence, 16S rRNA gene identity values underrepresent the genomic diversity within Pseudovibrio. This is also in line with the observation that $P$. japonicus NCIMB $14279^{\top}$ is closely related to $P$. denitrificans JCM $12308^{\top}$ based on the $16 \mathrm{~S}$ rRNA gene tree (99\% sequence identity) even though the two strains were highly dissimilar based on the OGP-based tree and ANI (81.4\%). It has been shown that ANI can confidently replace DNA-DNA 
hybridization $(\mathrm{DDH})$ values to delineate bacterial species with these two parameters showing strong $r^{2}$ correlation values of $>0.93$ [339, 340]. The $70 \% \mathrm{DDH}$ cut-off value for species delineation corresponds to $94-95 \%$ ANI [326]. However, so far no novel species have been published that use ANI as central evidence for species definition.

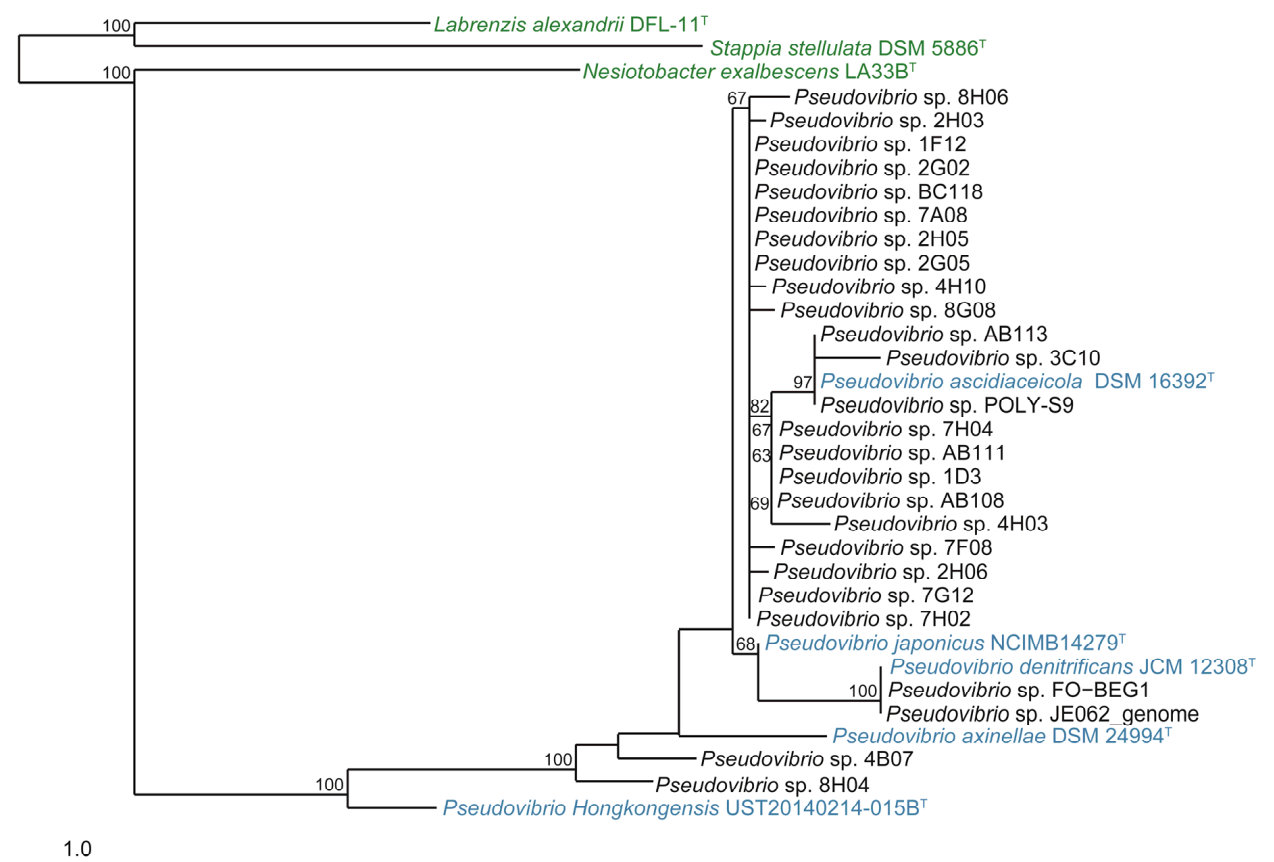

Figure 5.1 Phylogenetic tree based on 16S rRNA gene sequences from the 31 Pseudovibrio spp. and three close relatives that were included in the comparative genomics analysis. The Pseudovibrio type strains are depicted in blue and the close relatives are depicted in green. The tree was constructed in ARB by Maximum likelihood analysis using 1,000 iterations of bootstrapping. Bootstrap values $<50$ are not shown. The horizontal bar indicates the number of substitutions per site.

\section{Clustering by metadata and group-differences}

To identify variables that best explain the variation in the distribution of OGPs in the genomes, we performed a canonical correspondence analysis (CCA). Strains that are members of the outgroup (including $P$. hongkongensis UST20140214-015B ${ }^{\top}$ ) clustered separately from members of the Pseudovibrio genus ( $p=0.002$ ) (Figure 5.3A). Furthermore, we found clustering by geographic origin (Mediterranean, Caribbean, Pacific or Atlantic, $p=0.002$ ) (Figure 5.3B). The fact that geographic origin can partially explain the variation in OGPs suggests that in different geographic regions adaptation has occurred to different environmental conditions (e.g. temperature, salinity, local flora and fauna or nutrient availability). This is underlined 


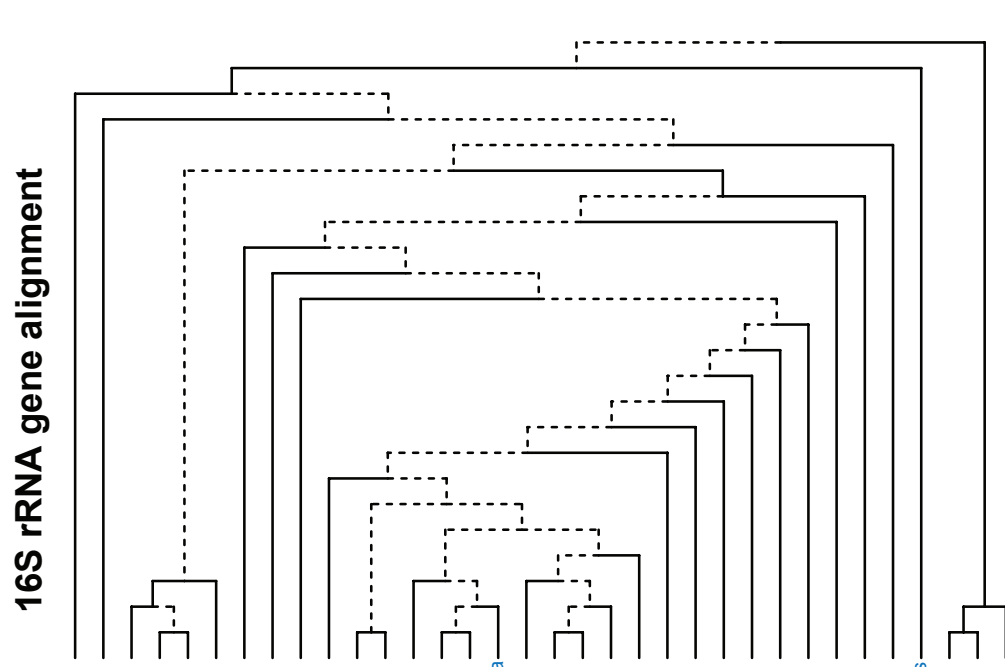

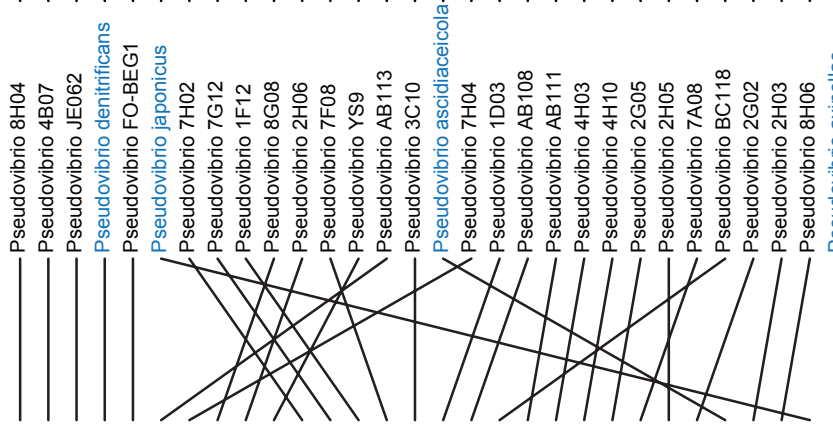

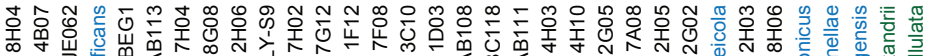

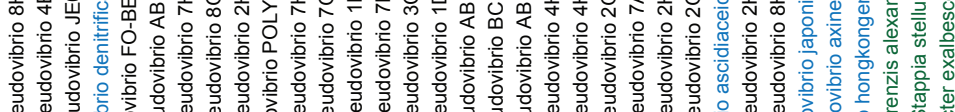

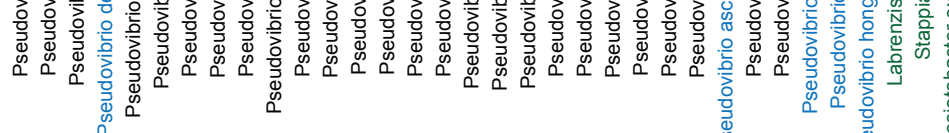

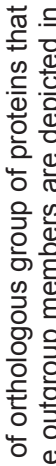

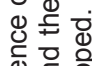

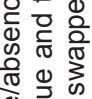

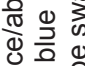

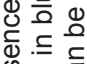

क⿺辶

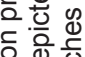

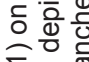

政

Did

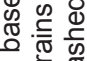

它 号

के

응 응

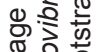

쓴 옹

雨通

它这.

휼

즈응응

응 웅

흠은

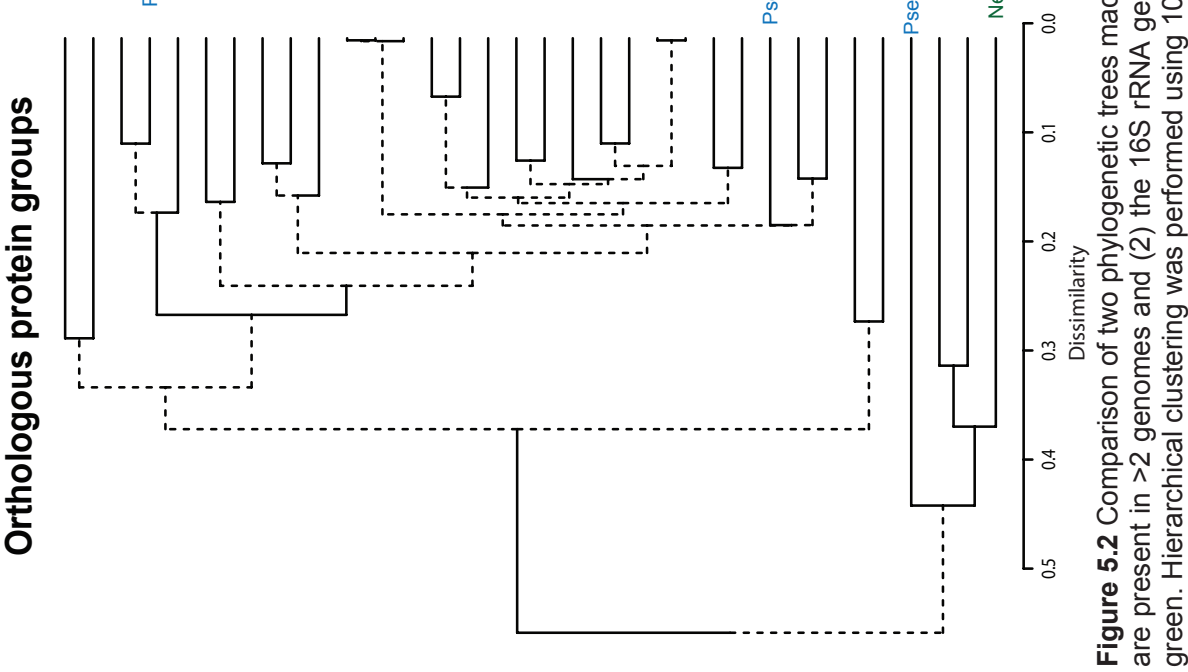


by the fact that clusters in two out of four geographic regions constituted different Pseudovibrio species. Most studies find that microbial composition and biogeography correlate in the aquatic environment, suggesting selective pressure of at least one environmental variable [344]. However, to what extent (trait) selection plays a role in the biogeography of Pseudovibrio spp., as opposed to the other suggested major processes such as drift, dispersal and mutation, remains to be elucidated. This question can be further investigated by attempting to link variation in OGPs to environmental variables, and reinforces the need for proper recording of metadata for environmental samples and derived microbial isolates [341, 342]. We also found that Pseudovibrio strains isolated near the coast of Spain clustered by sponge of origin (A. aerophoba, C. candelabrum or $P$. ficiformis, $p=0.006$ ) (Figure 5.3C). This might indicate that $P$ seudovibrio strains harbour (combinations of) functional genes that are associated with their ability to preferentially colonize certain sponge species. In turn, it might indicate that the Pseudovibrio strains selectively evolved in the sponge host after colonization. There is evidence that Pseudovibrio can be vertically transmitted [150], and in general various studies have demonstrated that at least in some cases, sponge-associated bacteria are transmitted vertically [343-346].

In order to identify differences in substrate utilization and product formation between the strains, the presence of metabolic pathways was investigated using the MetaCyc database as a reference. This database contains experimentally verified pathways and enzymes that are predominantly found in microorganisms and plants. We analysed pathways that contained at least three enzymes (Figure S5.1) and at least four enzymes with an EC number (Figure S5.2). In terms of metabolism, all members of the Pseudovibrio genus were highly similar i.e. across all genomes the same pathways were detected with differences between strains nearly always amounting to the presence and/or absence of single genes. As opposed to members of the outgroups, all Pseudovibrio spp. were predicted to be able to synthesize Coenzyme F420. Coenzyme F420 is best known as an essential coenzyme of methanogenesis [347]. However, since Pseudovibrio spp. are incapable of methanogenesis, F420 might be involved in SMB [348, 349] or other metabolic activities such as aerobic catabolism of 2,4,6-trinitrophenol or as an electron carrier to an $F_{420}$-dependent glucose-6-phosphate dehydrogenase [350-352]. In contrast to the three non-Pseudovibrio close relatives, all Pseudovibrio strains (including $P$. hongkongensis UST20140214-015B ${ }^{\top}$ ) were predicted to have the capacity to 
synthesize biotin (vitamin $B_{7}$ ). It has been suggested that sponges can benefit from the presence of bacteria that produce biotin, as they cannot synthesize the vitamin themselves [353]. High similarity between the Pseudovibrio spp. strains was also found with respect to the number of proteins assigned to COG functional categories (Table S5.3). The most pronounced difference between Pseudovibrio strains and the outliers was found for COG category "Mobilome: prophages, transposons", to which on average Pseudovibrio strains had twice the number of proteins assigned compared to the members of the outgroup.

Next, differences regarding the presence of individual OGPs was investigated with SIMPER in order to investigate which functional capacities define our analysed genomes when grouped according to geographic origin, sponge of origin and membership of the Pseudovibrio genus (Table S5.4). Genes encoding enzymes that have urease activity (EC 3.5.1.5) were exclusively predicted in the outgroup members, the three $P$. denitrificans strains and $P$. axinellae DSM $24994^{\top}$. The low abundance of nitrogen may be growth limiting in the oceans [354], and regeneration of urea (i.e. by urease), which is a waste product of many animals, may alleviate this restraint. Following this hypothesis, bacteria in sessile marine animals could enhance their growth by using the urea excreted by their host as nitrogen source [355]. Alternatively, the bacteria could contribute to the nitrogen budget of the host by fixation of environmental urea [356]. Given that the urease gene was detected in only a few (3 of 29) Pseudovibrio strains that reside in marine mammals, we do not expect it to play an important role in the hosts-symbiont relationship, or this gene only plays a role in specific hosts. SIMPER analysis also showed that OGPs corresponding to enzymes involved in vitamin $B_{12}$ (cobalamin) and vitamin $B_{1}$ (thiamin) biosynthesis were found in Pseudovibrio spp., but were absent in the outgroup members. Bacteria may be an essential contributor to the nutritional requirements of marine animals through production of these essential vitamins that the animals cannot synthesize themselves [151]. However, we found that only a few enzymes of the multi-enzyme pathway to produce vitamins $\mathrm{B}_{1}$ and $\mathrm{B}_{12}$ were present in Pseudovibrio indicating that the vitamins cannot be de novo synthesized. Regarding vitamin $\mathrm{B}_{1}$ biosynthesis, all Pseudovibrio strains lack a phosphotransferase (EC number: 2.7.4.7) and a thiazole tautomerase (5.3.99.10), both of which catalyse the final step to respectively produce pyrimidine and thiazole moieties that are required to form thiamine phosphate. Cobalamin cannot be synthesized de novo by Pseudovibrio spp. due to absence of 
essential enzymes such as the adenosylcobinamide phosphate synthase (EC number: 6.3.1.10) and the adenosyl-cobric acid synthase (6.3.5.10). No further characteristic functional traits could be ascribed to Pseudovibrio genomes grouped by environmental variables since a large number of the idiosyncratic OGPs were hypothetical proteins, or were proteins involved in routine processes.

\section{Symbiosis}

Eukaryotic-like proteins, virulence factors and quorum sensing

The interactions that are hypothesized to occur between Pseudovibrio spp. and the sponge host are still not well-understood. Eukaryotic-like proteins (ELPs) were first predicted to play a role in symbiosis when they were discovered to be highly prevalent in sponge-associated bacteria [261, 357-359]. Ankyrin-repeat proteins (ARPs), which are ELPs, were predicted to interfere with phagocytosis of Pseudovibrio by the sponge host [360]. It was also estimated that the average number of ARPs per sponge symbiont genome was between 10 and 40 as compared to an average of 2.5 ARPs in genomes of other symbiotic bacteria [361]. Our analysis predicted ARPs in 10 of 25 sponge-associated Pseudovibrio strains, with an overall average of $0.96 \pm 1.27$ ARPs per genome (Figure 5.4), which does rank them among known symbiotic bacteria based on their ARPs. Proteins containing SEL1 repeats are also predominantly found in eukaryotes, and these proteins have been predicted to be symbiotic factors by which resident bacteria can interact with the host cells [304, 362]. Our analysis predicted on average $6.9 \pm 1.5$ proteins with SEL1 repeats in the Pseudovibrio genomes. In the genomes of the Pseudovibrio strains that were not associated with marine animals (i.e. those isolated from sea water), that is Pseudovibrio japonicus NCIMB14279 ${ }^{\top}$ and Pseudovibrio denitrificans JCM $12308^{\top}, 9$ and 8 proteins with SEL1 repeats were detected, respectively. ELPs with tetratricopeptide repeats (TRPs) have been reported to participate in the delivery of virulence factors to the host cell [363]. On average we detected $17.6 \pm 1.1$ proteins with TRPs in the Pseudovibrio genomes. We also detected on average $14 \pm 3.4$ proteins with invasion associated locus $B$, which has been reported to be involved in host cell invasion [364, 365]. Furthermore, in each Pseudovibrio genome two proteins were predicted that contain a TadE-like domain, suggesting that these might be involved in adherence of Pseudovibrio to the host cells [366]. We investigated whether the LuxR/LuxI quorum sensing system was present in the Pseudovibrio 
genomes. The enzyme encoded by Luxl catalyses the final step in the production of $\mathrm{N}$-acyl homoserine lactone (NHL). NHL can bind to the protein product of LuxR,
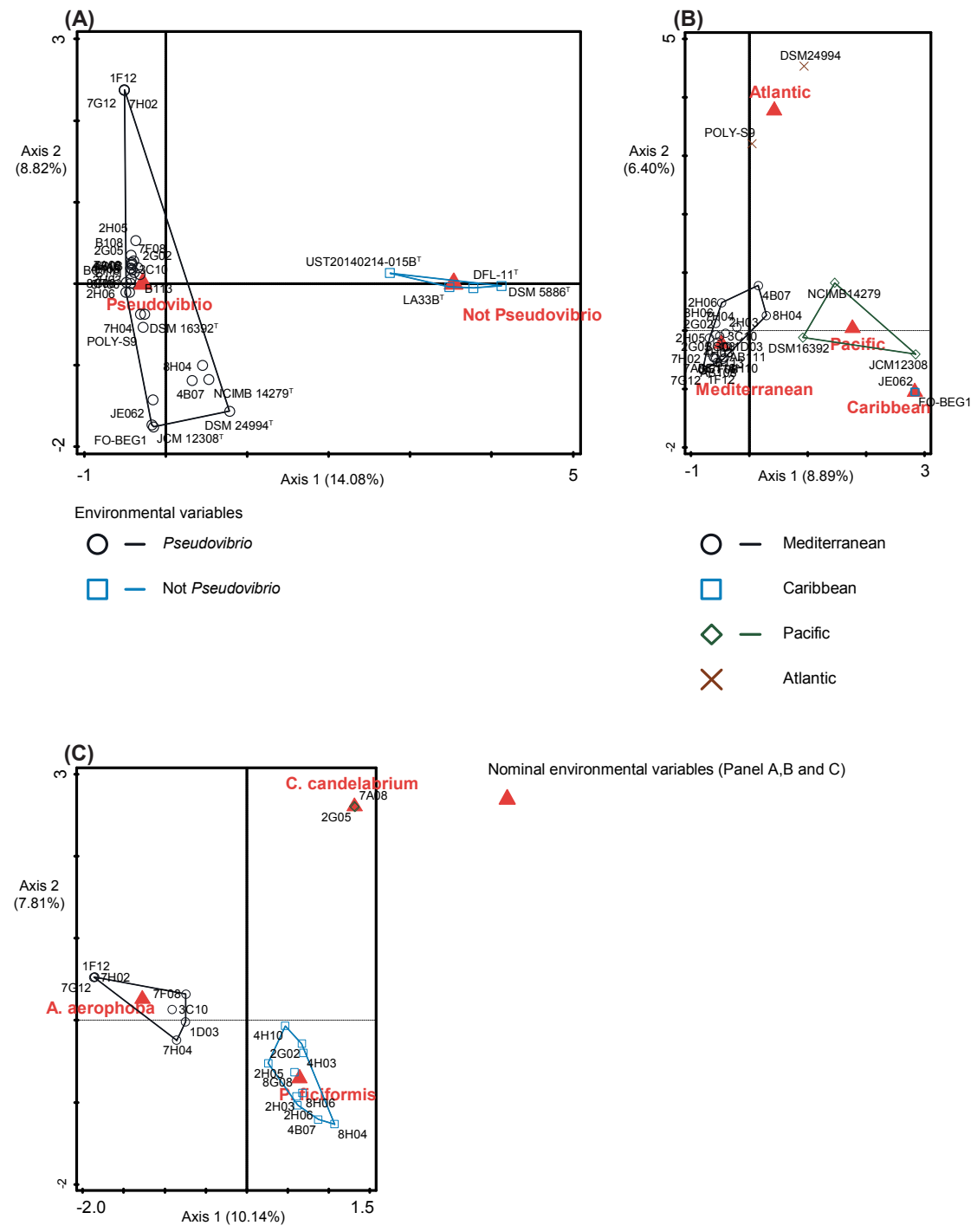

Nominal environmental variables (Panel A,B and C)

Environmental variables

$$
\begin{aligned}
& O-\text { A. aerophoba } \\
& \square-\text { P. ficiformis } \\
& \diamond \quad \text { C. candelabrum }
\end{aligned}
$$

Figure 5.3 Canonical correspondence analysis was used to investigate which environmental variables could explain the variation in orthologous protein group composition amongst the genomes. All clustering shown in this figure is statistically significant $(p<0.01)$. Panel $\mathbf{A}$ shows results based on grouping of the genomes according to membership of the Pseudovibrio genus. In panel $\mathbf{B}$ the Pseudovibrio genomes are grouped according to their geographic origin. In panel C the Pseudovibrio strains that were isolated at the coast of Spain are grouped according to their sponge of origin. 
which together activate transcription of genes involved in NHL biosynthesis (autoinduction) as well as a wide array of other genes [367, 368]. NHL is secreted by the bacterium; therefore, as the population density increases, the concentration of $\mathrm{NHL}$ will increase, resulting in activation of gene expression across the bacterial community. In each Pseudovibrio genome we detected $\geq 10$ copies of the LuxR gene whereas in none of the genomes Luxl was detected. An absence of Luxl suggests that Pseudovibrio strains are dependent on quorum sensing molecules produced by neighbouring bacteria for expression of LuxR/NHL-controlled genes, which has been previously hypothesized based on the genomes of Pseudovibrio sp. FO-BEG1 and Pseudovibrio sp. JE062 [151, 369]. The genes regulated by quorum sensing may be involved in production of secondary metabolites (e.g. against microbial competitors or host pathogens) or biofilm formation [370, 371]. In 29 of the 31 Pseudovibrio genomes analysed here we detected a cluster of genes putatively involved in biogenesis of proteinaceous extracellular fibres called curli. These extracellular curli are involved in biofilm formation, and as such may be contribute to sponge colonization [372]. Compared to the Pseudovibrio genomes, the genomes of the outgroup members encoded similar numbers of ELPs, LuxR enzymes, proteins with invasion associated locus $B$ and proteins containing the TadE-like domain. However, no outgroup member encoded proteins involved in curli fibre biosynthesis.

\section{Secretion systems}

The distribution of Type III secretion systems (T3SSs), Type IV secretion systems (T4SSs) and Type VI secretion systems (T6SSs) was investigated (Figure 5.4), because these systems have been ascribed important roles in a range of symbiotic interactions. As query we used genomic regions of Pseudovibrio genomes in which the T3SS, T4SS, T6SS-I and T6SS-I were previously detected [305]. All three types of secretion systems allow bacteria to deliver effector molecules into a target cell via needle-like structures in the cellular membrane [373-375]. Besides effector molecules, T4SSs can also translocate genetic material to the target cell. In all Pseudovibrio genomes, except the genome of $P$. japonicus NCIMB14279 ${ }^{\top}$, at least two of these secretions systems were detected. Interestingly, in $P$. japonicus NCIMB $14279^{\top}$ not a single of the aforementioned secretion systems was found indicating that the strain might lack equipment to form symbiotic relations. This notion is in line with the fact that $P$. japonicus NCIMB $14279^{\top}$ was isolated from the open 
sea, and that members of this species were so far never isolated from marine animals. On the other hand, the notion is contradicted by $P$. japonicus spp. being detected more often in sponge 16S rRNA gene datasets as compared to sea water datasets (Figure 5.5). T6SS-I and/or T6SS-II clusters were detected in all

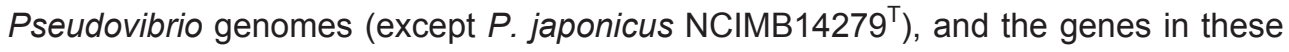
clusters were all homologous and showed perfectly conserved synteny (Table S5.5). The fact that the clusters are conserved in Pseudovibrio strains from diverse geographical locations and species suggests that they are important for survival and propagation. T3SS clusters were limited to Pseudovibrio strains (27/28) belonging to the species $P$. ascidiaceicola and $P$. denitrificans, whereas T4SSs were detected in only 10 out of all Pseudovibrio genomes. These results suggest Pseudovibrio spp. developed different strategies to carve a niche in a competitive environment. Romano and collegues [305] carried out a more detailed analysis of these clusters for Pseudovibrio sp. FO-BEG1 and Pseudovibrio sp. JE062, as well as for 10 other Pseudovibrio strains, by analysing which effector molecules are putatively delivered into host cells. It was predicted that T3SSs were mainly used to reduce phagocytosis and to block the inflammatory response of the host, that T4SSs were mainly involved in DNA mobilization, and that T6SSs might be involved in evasion of the host immune response, or they could have an antagonistic role towards other bacteria. Only a single secretion system, namely the T4SS in $P$. hongkongensis UST20140214$015 \mathrm{~B}^{\top}$, was detected in the outgroup members. The members of the outgroup (except $P$. hongkongensis UST20140214-015B ${ }^{\top}$ ) do not belong to genera that are implicated in forming symbiotic relations. In addition, all outgroup members, except Labrenzia alexandrii DFL-11 ${ }^{\top}$ that was washed from the dinoflagellate Alexandrium Iusitanicum [376], were not isolated from a host. Hence, the absence of symbiosisrelated gene clusters in their genomes (e.g. those encoding curli fibre formation and secretion systems) likely indicates that these bacteria are not committed to forming symbiotic relations. 


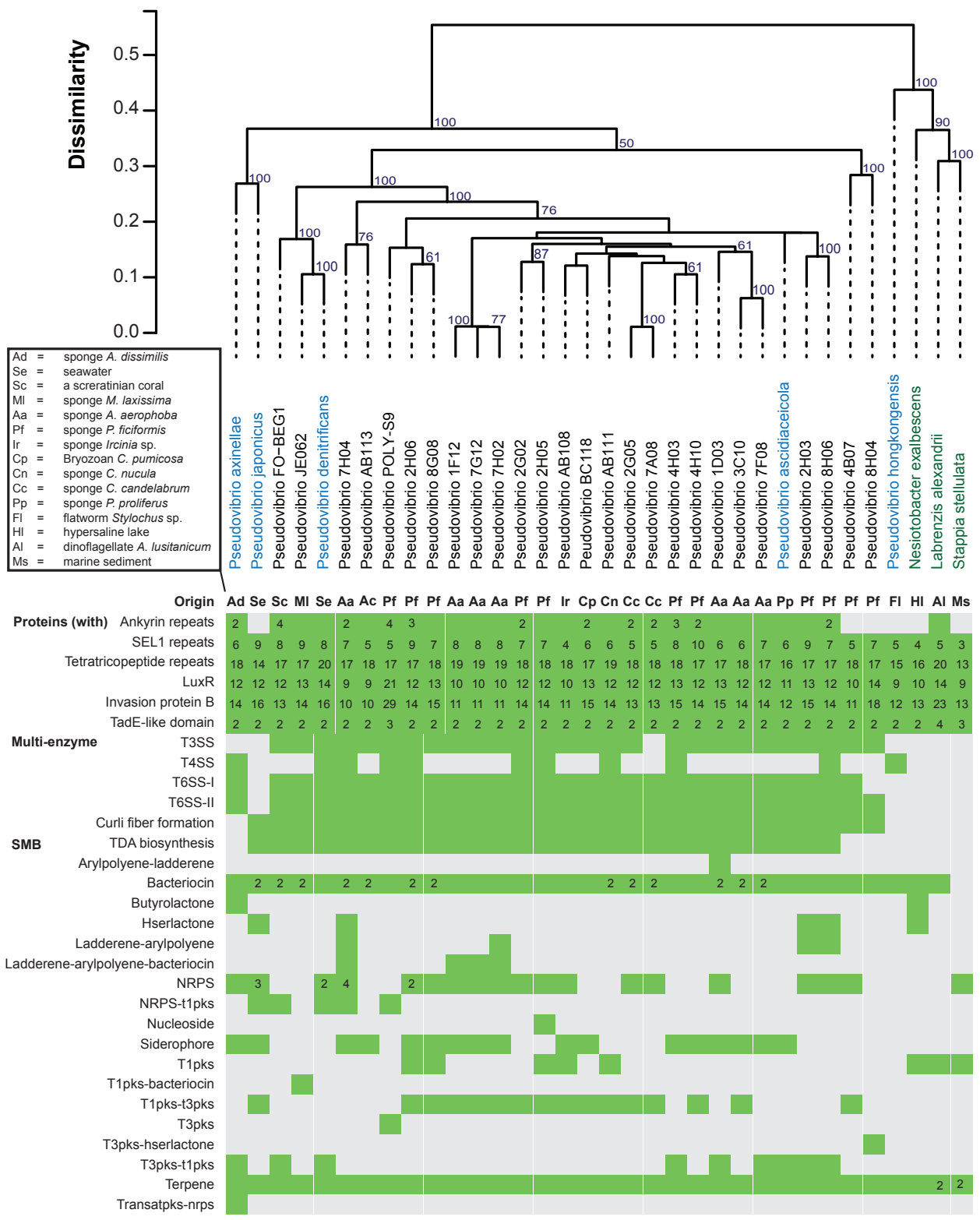

Figure 5.4 Symbioses-related proteins, secretions systems and secondary metabolite biosynthesis (SMB) clusters that were detected in the genomes of the Pseudovibrio spp. and their close relatives. Squares are coded green if the corresponding protein(s) was detected. Numerical values were included if functions were detected more than once. The tree on top was made by hierarchical linkage clustering based on presence/absence of orthologous group of proteins that are present in $>2$ genomes. Pseudovibrio type strains are depicted in blue and the close relatives are depicted in dark green. Hierarchical clustering was performed using 10,000 iterations of bootstrapping. 


\section{Secondary metabolite biosynthesis clusters}

Pseudovibrio spp. have received high interest as sources of novel bioactive compounds, and especially antimicrobials [309]. Not only are novel bioactive compounds interesting from a clinical perspective but they may also provide insights into possible host-symbiont relations. Sponges in part depend on the production of bioactive active compounds by associated microorganisms for defense against predation and disease $[123,125]$. Tropodithietic acid (TDA) is such a compound that has antibacterial activity, and was found to be produced by Pseudovibrio sp. D323 [161]. TDA has a strong inhibitory activity against a range of marine bacteria, and hence is proposed to protect the host from unwanted microbial colonization [307]. It has been previously shown that Pseudovibrio sp. FO-BEG1 and Pseudovibrio sp. JE062 can produce TDA, which was linked to the presence of the tdaA-tdaF biosynthetic gene cluster in the respective genomes [151]. Here we found the tdaAtdaF biosynthetic cluster in 27 of 31 Pseudovibrio genomes which would suggest that TDA production is an important feature of the genus Pseudovibrio (Figure 5.4). It cannot be determined if the three Pseudovibrio strains without the tdaA-tdaF cluster also lack TDA resistance as the resistance mechanism is not yet understood; however, it is tempting to speculate that these strains can benefit in the sponge from co-resident TDA-producing Pseudovibrio spp. Further potential for SMB was analysed by application of the antiSMASH pipeline [336]. Eighteen of 43 types of SMB clusters defined by antiSMASH were detected in the Pseudovibrio genomes, with bacteriocin, NRPS, siderophore, T1pks-t3pks, T3pks-t1pks and terpene SMB clusters being detected in $>5$ genomes (Figure 5.4). At least one bacteriocin biosynthesis gene cluster was identified in all Pseudovibrio genomes, except for Pseudovibrio axinellae DSM $24994^{\top}$. The main role of bacteriocins, which are ribosomally synthesized antibiotic peptides, is to provide their producers with a competitive advantage by inhibiting bacterial growth [377, 378]. Therefore, similar to TDA, bacteriocins could serve Pseudovibrio to establish a niche in the sponge host, and at the same time protect the host against pathogen invasion. So far, few bacteriocins have been identified in the marine environment [379], and only one study has identified novel bacteriocins in a sponge [380]. AntiSMASH detected a terpene biosynthesis gene cluster in all Pseudovibrio genomes. Recently, via mining of bacterial genomes, it has become clear that terpenes are not only regularly produced by plants and fungi but also by a large number of bacteria [381]. Terpenes 
may give Pseudovibrio a competitive advantage due to antimicrobial activity [382, 383]. Alternatively, the fragrance of the terpenes might be an addition to spongederived secondary metabolites that are designed to repel predators [243, 384, 385]. However, terpenes typically have limited water solubility. For the SMB clusters detected by antiSMASH in Pseudovibrio spp., no more than $26 \%$ of the genes in a cluster showed similarity to genes in known clusters. Taken together, these findings highlight Pseudovibrio spp. as a largely untapped resource for the discovery of novel compounds with potentially clinical significance.

\section{Antibiotic resistance}

Antibiotic resistance profiles were determined for 27 out of 31 Pseudovibrio strains that were analysed in this study (Table S5.6). All 27 strains were resistant to vancomycin, lincomycin, polymyxin $\mathrm{B}$ and D-cycloserine, and these resistance phenotypes could not be linked to the presence of known resistance genes. Therefore, resistance to these antibiotics is probably intrinsic. Twenty-one of 27 strains were resistant to tetracycline, which could not be linked to the presence of resistance genes in the genomes. All strains were either resistant or intermediately resistant to the $\beta$-lactam antibiotics ampicillin and penicillin, which could be explained by the class $A$ and class $B \beta$-lactamases that were predicted to be encoded in their genomes (Table S5.7). The predicted class A $\beta$-lactamase encoded in 22 Pseudovibrio ascidiaceicola genomes was highly similar ( $\geq 95 \%$ amino acid identity) to blapsv-1 from $P$. ascidiaceicola $1 D 03$ of which the resistance function has been experimentally verified by functional metagenomics (Chapter $\mathbf{4}$ of this thesis). Within the Pseudovibrio genus, almost all class A $\beta$-lactamases clustered by species (Figure S5.3). The exception was a distinct class A $\beta$-lactamase (8H04_2067) predicted in Pseudovibrio sp. strain $8 \mathrm{H} 04$ of which the closest experimentally verified $\beta$-lactamase was blapsv-1. However, as previously deduced, Pseudovibrio sp. strain $8 \mathrm{H} 04$ is expected to represent a distinct novel Pseudovibrio species as well. These results, together with the absence of associated mobilizing elements, suggest that the class A $\beta$-lactamases are not prone to horizontal gene transfer, and that diversity within this group of enzymes is predominately achieved by speciation. The $\beta$-lactamases in these bacteria could function as a defense against $\beta$-lactam antibiotics that are produced by other micro-organisms co-inhabiting sessile marine animals. Still, these enzymes could also have other roles such as in disruption of cell signalling [299]. 


\section{Environmental distribution of Pseudovibrio}

Using the IMNGS platform (www.imngs.org) we investigated in which percentage of environmental 16S rRNA gene datasets in the SRA database the Pseudovibrio type strains were detected (Figure 5.5). IMNGS divides the 16S rRNA gene datasets in 105 categories according to which biological niche was sampled. The highest percentage of datasets containing Pseudovibrio spp. was found for the category sea squirts $(20.8 \%)$, followed by datasets acquired from sponges $(13.7 \%)$ and symbionts (7.6\%). Pseudovibrio spp. were only detected in 16S rRNA datasets from marine habitats or from generalised categories that can also contain datasets from marine habitats. This is consistent with the notion that Pseudovibrio spp. have exclusively been isolated from the marine environment i.e. they depend on saltwater to thrive. The fact that Pseudovibrio spp. were detected more often in 16S rRNA gene datasets from sessile marine animals as opposed to aquatic environments suggests that indeed Pseudovibrio spp. form symbiotic relations. $P$. ascidiaceicola, $P$. denitrificans and $P$. japonicus were detected in $>6 \%$ of the $16 \mathrm{~S}$ rRNA gene datasets from at least two categories, whereas $P$. axinellae and $P$. hongkongensis were never detected in $>1 \%$ of the datasets of any category.

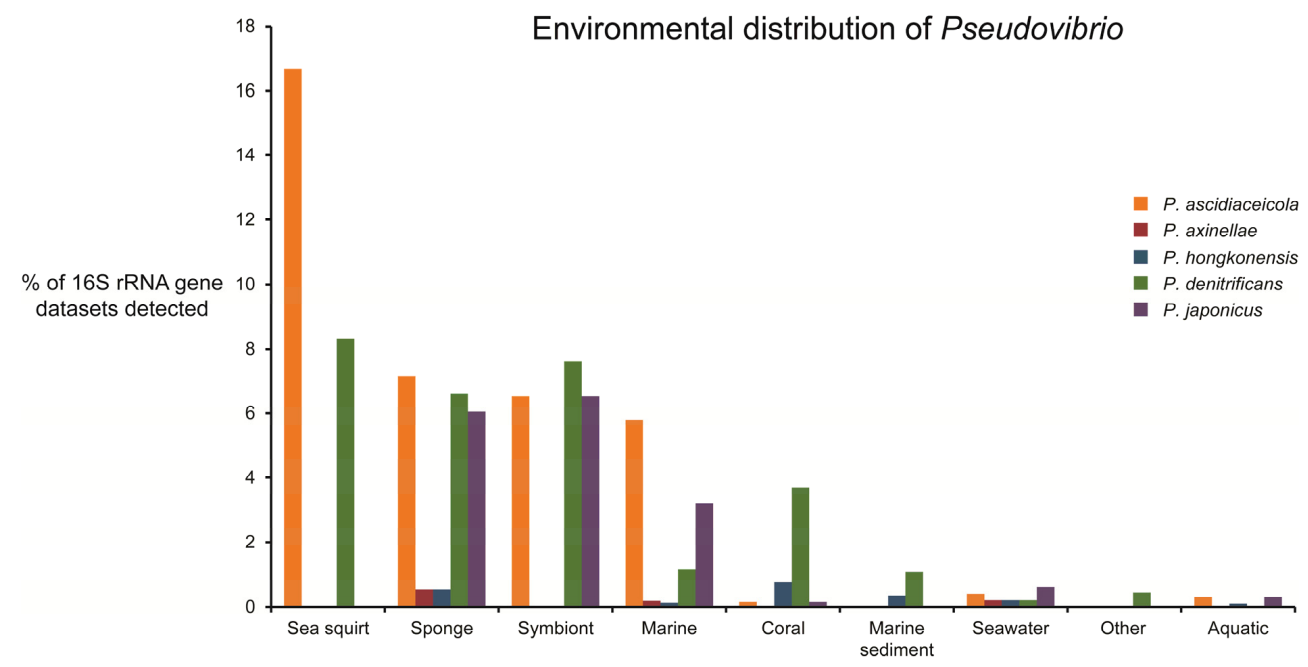

Figure 5.5 Percentage of 16S rRNA gene amplicon datasets in the SRA database in which the Pseudovibrio type strains were detected. The analysis was performed by the IMNGS platform (https://www.imngs.org/about/), which divides the datasets in 105 categories according to which biological niche was sampled. Full-length 16S rRNA gene sequences of the type strains were used as query. The similarity threshold was set at $99 \%$ nucleotide identity. All environmental categories are shown in which at least one type strain was detected at $>0.1 \%$ relative abundance. 


\section{Conclusions}

Our analysis of a total of 31 Pseudovibrio genomes revealed that, discounting Pseudovibrio hongkongensis UST20140214-015B ${ }^{\top}$, the Pseudovibrio strains are highly similar in term of metabolic capacity, with striking differences regarding the presence of specific SMB clusters. We identified various genomic elements that are highly conserved within the genus, all of which we expect to give Pseudovibrio a competitive advantage as a sponge (or other marine animal) symbiont. These conserved elements encompassed systems involved in immune evasion (T4SSs and T6SSs), SMB clusters which products might serve to inhibit growth of competitors (TDA, bacteriocin and terpene biosynthesis clusters), and a range of other symbiosis factors. The importance of these elements for Pseudovibrio survival is underscored by their conservation across Pseudovibrio spp., and across strains isolated from diverse geographic locations. Interestingly, based on gene composition, Pseudovibrio strains from the Mediterranean Sea clustered by sponge of origin, suggesting hostspecific colonization or adaptation. We also showed that Pseudovibrio spp. are predominantly detected in 16S rRNA gene datasets of sessile marine animals. Taken together, these results support the hypothesis that Pseudovibrio spp. have evolved as symbionts of sponges and other marine invertebrates.

\section{Acknowledgements}

This work was supported by the European Union through the EvoTAR project (Grant agreement no. 282004) and by a grant of BE-Basic-FES funds from the Dutch Ministry of Economic Affairs (project 7.1.5). 


\section{Supplementary information}

\section{Supplementary Tables and Figures}

Tables S5.1, S5.2, S5.3, S5.4, S5.5, S5.6 and S5.7 and Figures S5.1 and S5.2 are available upon request.

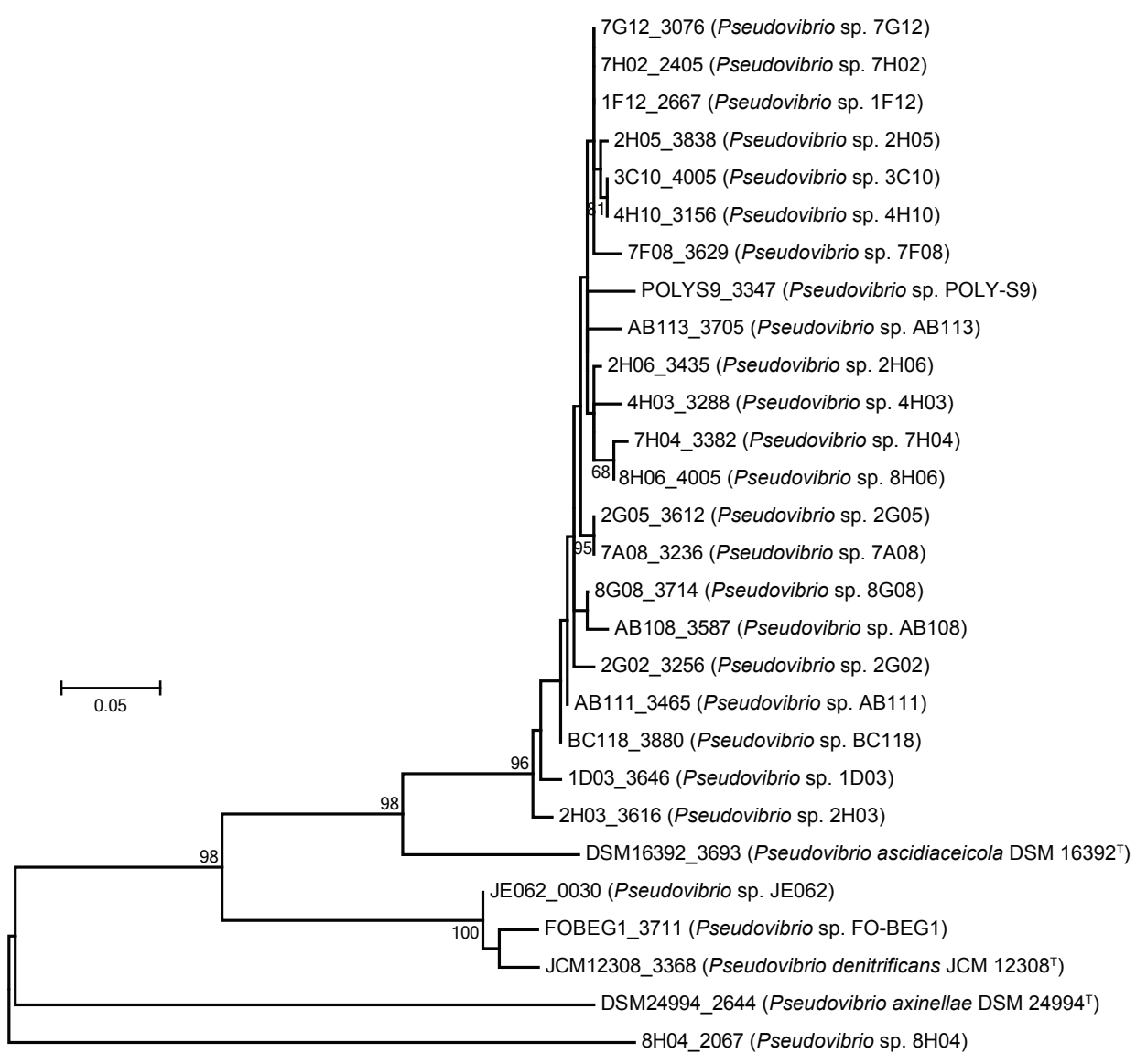

Figure S5.3 Maximum Likelihood Tree based on protein sequences of the 28 class A B-lactamases that were predicted in the Pseudovibrio genomes. The locus tags of the proteins are given with between brackets the corresponding Pseudovibrio strain. The tree was constructed in MEGA using 1,000 iterations of bootstrapping. Bootstrap values $<50$ are not shown. The horizontal bar indicates the number of substitutions per site. 



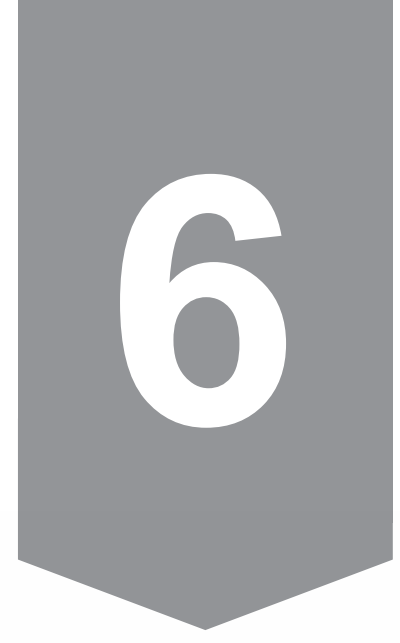

High throughput cultivation-based screening on the MicroDish platform allows targeted isolation of antibiotic resistant human gut bacteria 


\section{Abstract}

The emergence of bacterial pathogens that are resistant to clinical antibiotics poses an increasing risk to human health. The most important reservoir from which bacterial pathogens can acquire resistance is the human gut microbiota. However, to date, a large fraction of the gut microbiota remains uncultivated and has been little-studied with respect to its reservoir-function. Here, our aim was to isolate yet uncultivated resistant gut bacteria by a targeted approach. Therefore, faecal samples from 20 intensive care patients who had received prophylactic antibiotic treatment (selective digestive decontamination [SDD], i.e. tobramycin, polymyxin $E$, amphotericin $B$ and cefotaxime) were inoculated anaerobically on MicroDish porous aluminium oxide chips placed on top of poor and rich agar media, (including media supplemented with the SDD antibiotics). Biomass growing on the chips was analysed by 165 ribosomal RNA gene amplicon sequencing, showing large inter-individual differences in bacterial cultivability, and enrichment of a range of taxonomically diverse operational taxonomic units (OTUs). Furthermore, growth of Ruminococcaceae (2 OTUs), Enterobacteriaceae (6 0 OTUs) and Lachnospiraceae (4 OTUs) was significantly inhibited by the SDD antibiotics. Strains belonging to 16 OTUs were candidates for cultivation up to pure culture as they shared $\leq 95 \%$ sequence identity with the closest type strain and had a relative abundance of $\geq 2 \%$. Six of these OTUs were detected on media containing SDD antibiotics, and as such were prime candidates to be studied with regards to antibiotic resistance. One of these six OTUs was obtained in pure culture using targeted isolation. This novel strain, which was initially classified as member of the Ruminococcaceae, was later found to share $99 \%$ nucleotide identity with the recently published Sellimonas intestinalis BR72 ${ }^{\top}$. In conclusion, we showed that high-throughput screening of growth communities can guide targeted isolation of bacteria that may serve as reservoirs of antibiotic resistance. 


\section{Introduction}

The emergence of bacterial pathogens that are resistant to most clinical antibiotics is an increasing threat to public health. A common route through which pathogens can acquire resistance is by genetic exchange with human-associated bacteria, and especially the gut microbiota. Indeed, it has been shown that the commensal gut microbiota harbours diverse resistance genes [104, 202], and that these genes can be acquired by (opportunistic) pathogens [80]. Horizontal gene transfer (HGT) is considered the main mechanism by which resistance genes are disseminated, and it has been shown that HGT events occur exceedingly more often in the gut microbiota than in other environments with complex bacterial communities [187]. Novel resistance determinants are typically described once bacteria are obtained in pure culture. On the other hand, resistance genes carried by yet uncultivated gut bacteria appear to be largely uncovered, which is reinforced by the observation that functional metagenomics studies of human gut microbiota consistently yield novel resistance genes [185, 386]. This "black box" of little-studied uncultivated bacteria has been estimated to constitute $40-70 \%$ of gut microbes [387, 388]. Even though application of culture-independent methods (e.g. functional metagenomics) has provided us with useful insights into uncultivated bacteria, their cultivation will be essential to comprehensively study the antibiotic resistance phenotype, and the potential roles and mechanisms of these bacteria in antibiotic resistance dissemination. Nowadays, to isolate members of yet uncultivated taxa, innovative culturing techniques that apply high-throughput screening and/or better simulate the natural environment of these bacteria are increasingly being used. Recent methodical advances include cultivation inside chambers placed in the native environment [90, 91], the use of customdesigned media [95], application of multi-well micro culture chips [89], highthroughput identification of isolates [85], and microfluidic cultivation [389]. Furthermore, a recent study by Rettedal and co-authors combined high-throughput sequencing with selective cultivation conditions, allowing to cultivate previously uncultured species from the human gut by a targeted approach [390]. To this end, the authors used, among other criteria, the "most wanted" list of microbial taxa that has recently been introduced in order to guide efforts towards the cultivation of human gut bacteria [391]. In short, the most wanted list contains human-associated bacterial taxa of which the genome has not yet been sequenced, not considering 
whether members of these taxa from other environments might already have been sequenced. High priority most wanted taxa were defined as those of which the $16 \mathrm{~S}$ rRNA centroid read shared less than $90 \%$ identity with either the GOLD-Human or Human Microbiome Project (HMP) strains, and which were detected in at least 20\% of samples from any body habitat analysed. Medium priority taxa are those that share between $90 \%$ and $98 \%$ identity with the same habitat prevalence threshold.

Antibiotics are generally administered upon detection of an infection. In addition, in most Dutch hospitals, patients who are admitted to the intensive care unit (ICU) receive prophylactic antibiotic therapies, of which selective decontamination of the digestive tract (SDD) is currently the most common treatment. SDD combines the application of tobramycin, polymyxin $E$ and amphotericin $B$ in the oropharynx and gastrointestinal tract with a short systemic administration of a third-generation cephalosporin. The therapy aims to eradicate potential pathogens such as Staphylococcus aureus, Pseudomonas aeruginosa Enterobacteriaceae and pathogenic fungi while maintaining the anaerobic members of the microbiota [392]. SDD therapy has been shown to decrease infections and mortality of ICU patients [393, 394]. Although a meta-analysis showed that SDD therapy resulted in a decrease in resistance carriage with respect to cultivable bacteria [395], a recent case study [7] and a more extensive follow-up with 13 ICU patients [396] indicated that prophylactic therapy may in fact increase resistance carriage among mostly uncultivated anaerobic gut residents. It was speculated that the expanded resistome, i.e. the collection of all resistance genes in a bacterial community [74], might thereby increase the risk of future pathogens becoming resistant. Indeed, the risk that pathogens develop antibiotic resistance is a major concern that has prohibited wide implementation of prophylactic therapies [395]. In view of the above, it is clear that the role of uncultivated anaerobic bacteria in the emergence of resistance pathogens merits deeper investigation.

In this study, we aimed to identify and isolate potential reservoir strains for antibiotic resistance in the anaerobic microbiota of the human gut. Therefore, faecal material from 20 Dutch ICU patients was inoculated on poor and rich agar media under anoxic conditions. These media were prepared without antibiotics, or supplemented with the antibiotics that the patients received as part of their SDD therapy. Bacteria were cultivated on the MicroDish porous aluminium oxide (PAO) chip that facilitates efficient parallel processing of a large number of samples, and 
reduces potential inhibition of bacterial growth by agar [89]. Chips that were placed on top of different media solidified with agar were inoculated with faecal suspensions, and bacterial biomass was investigated by $16 \mathrm{~S}$ ribosomal RNA (rRNA) gene amplicon sequencing, based on which growth patterns were analysed and target species were identified for cultivation up to pure culture. Accordingly, we isolated a close relative of Sellimonas intestinalis $B R 72^{\top}$ that grew on top of the PAO chip on agar media containing tobramycin, cefotaxime and polymyxin $\mathrm{E}$, and that could serve as a antibiotic resistance reservoir.

\section{Materials and Methods}

\section{Sample collection}

Faecal samples were collected from 20 patients no later than five days after admission to the ICU at Utrecht Medical Center, Utrecht, the Netherlands. During this period, the patients received SDD therapy [7]. The SDD protocol was reviewed and approved by the institutional review board of the University Medical Center Utrecht. Faecal samples were collected upon defecation and stored at $4{ }^{\circ} \mathrm{C}$ for $30 \mathrm{~min}$ to $4 \mathrm{~h}$, after which an aliquot of the sample (approximately $0.5 \mathrm{~g}$ ) was suspended in $5 \mathrm{ml}$ of anaerobic PBS ( $\mathrm{pH}$ 7.0). Subsequently, $2 \mathrm{ml}$ of the suspension was transferred to an anaerobic bottle containing $4 \mathrm{ml}$ of PBS, $25 \%(\mathrm{v} / \mathrm{v})$ glycerol, $0.5 \mathrm{~g}$ resazurin and $0.5 \mathrm{~g}$ cysteine. To preserve anaerobic conditions, a few drops of titanium citrate (100 mM) were added to the bottle before storage at $-80{ }^{\circ} \mathrm{C}$.

\section{Cultivation conditions}

A high throughput cultivation technique using the MicroDish PAO chip (MicroDish, Utrecht, the Netherlands) was applied. Faecal bacteria were cultured on ethanolsterilized PAO chips on top of two different media: (i) GIFU anaerobic agar media (GAM) (Hyserve, Uffing, Germany), and (ii) bicarbonate-buffered anaerobic media (referred to in the text as CP medium) [397] supplemented with $1.5 \%(\mathrm{w} / \mathrm{v})$ agar and $1 \%(\mathrm{v} / \mathrm{v})$ faecal supernatant. The faecal supernatant was prepared from a pool of faecal samples obtained from three healthy volunteers who had not received antibiotics for at least six months. In brief, equal amounts of faecal sample from the three volunteers were added to anaerobic PBS $(\mathrm{pH} \mathrm{7.0)}$ to a final concentration of $25 \%(w / v)$. Subsequently, the mixture was centrifuged at 14,000 rpm for $30 \mathrm{~min}$, after which the supernatant was transferred to an anaerobic bottle $\left(\mathrm{N}_{2} / \mathrm{CO}_{2}-80: 20, \mathrm{v} / \mathrm{v}\right)$ and autoclaved. All samples were cultivated on agar media both in the presence and 
in the absence of the SDD cocktail of antibiotics $(25 \mu \mathrm{g} / \mathrm{ml}$ tobramycin, $5 \mu \mathrm{g} / \mathrm{ml}$ polymyxin $\mathrm{E}$ and $10 \mu \mathrm{g} / \mathrm{ml}$ cefotaxime; the antifungal drug amphotericin $\mathrm{B}$ was not included in this study). Five $\mu$ faecal suspension was applied per PAO chip. Inocula consisted of undiluted and 100-fold diluted cryopreserved faecal suspension. In addition, 10-fold sample dilutions were included for three patients (designated 210, 131 and 148) in order to study the effect of dilution on bacterial growth. PAO chips on top of GAM agar and CP agar were harvested two and three days after inoculation under anoxic conditions at $37^{\circ} \mathrm{C}$, respectively. Upon harvesting, PAO chips with bacterial growth were placed in an Eppendorf tube containing $1 \mathrm{ml}$ of anaerobic PBS $(\mathrm{pH}$ 7.0). The tube was vortexed for $30 \mathrm{~s}$ to dissociate the cells from the PAO chip. Subsequently, the suspension was split into two fractions; one fraction was used for DNA extraction whereas the other fraction was added to an anaerobic bottle containing glycerol (final concentration: 25-30\%) in PBS, and stored at $-80{ }^{\circ} \mathrm{C}$. Biological duplicates were analysed for each growth community.

\section{DNA extraction and 16S rRNA gene amplicon sequencing}

Barcoded 16S rRNA gene amplicon sequencing was used to investigate the bacterial composition of the faecal samples and of the growth communities on the PAO chips. The cells in these samples were lysed and (cellular) debris was removed with an adapted bead beating protocol [398]. In case of cryo-preserved faecal material, 500 $\mu \mathrm{l}$ of sample was added to a screw-cap tube that already contained $0.5 \mathrm{~g}$ of $0.1 \mathrm{~mm}$ zirconium beads (Biospec Products, Bartlesville, United States) and three $5 \mathrm{~mm}$ glass beads (Biospec Products). Subsequently, $300 \mu$ STAR buffer (Roche, Basel, Switzerland) was added, after which the contents of the tube was homogenized in the Precellys 24 (Bertin Technologies, Montigny-le-Bretonneux, France) at $5.5 \mathrm{~ms}$ (3 rounds of $1 \mathrm{~min}$ ). The sample was then incubated at $95{ }^{\circ} \mathrm{C}$ at $100 \mathrm{rpm}$ for $15 \mathrm{~min}$. Particles were spun down at $4{ }^{\circ} \mathrm{C}$ at $>10,000 \mathrm{~g}$ for $5 \mathrm{~min}$, and subsequently the supernatant was transferred to a fresh tube for DNA extraction. The DNA yield was improved by another two iterations of beat-beating that started with re-suspending the pellet in $300 \mu \mathrm{S}$ STAR buffer. In case of bacteria suspended in PBS (i.e. the growth communities), $150 \mu \mathrm{l}$ of sample was processed by identical methods except at a smaller scale. Therefore, $0.25 \mathrm{~g}$ of $0.1 \mathrm{~mm}$ zirconium beads and three $2.5 \mathrm{~mm}$ glass beads were added to the screw-cap tube, and STAR buffer was used in portions of $150 \mu$ l. Following the bead beating protocol, DNA was extracted from 250 
$\mu \mathrm{l}$ of the combined supernatants using the Maxwell 16 Tissue LEV total RNA purification kit starting from the post lysis step (Promega, Madison, United States). 16S rRNA gene amplification, which also attached the barcodes, was done with a 2step PCR protocol (Table S6.1) [399]. The product from the second PCR step was analysed on a $1 \%$ agarose gel and purified using the CleanPCR Kit (GC Biotech, Alphen aan den Rijn, Netherlands) according to manufacturers' instructions. The DNA concentration was measured by Qubit ${ }^{\circ} 2.0$ (Thermo Fisher Scientific). Subsequently the sample was included in a pool that in total contained 48 equimolarly mixed samples. The pool of samples, which constituted a library, was sent for Illumina paired end MiSeq sequencing (2 x 300 bp) at GATC Biotech (Constance, Germany). In total, eight libraries were sent MiSeq for sequencing. Technical replicates at the level of $16 \mathrm{~S}$ rRNA amplicon sequencing were analysed for the original faecal samples.

\section{Processing of 16S rRNA gene amplicon data, statistical analyses and detection of most wanted and novel species}

The 16S rRNA gene amplicon data were analysed using the NG-tax pipeline [400]. In short, NG-tax initially defines operational taxonomic units (OTUs) as clusters of 16S RNA gene amplicons that share $100 \%$ nucleotide identity. Subsequently, the OTUs are expanded by including 16S RNA gene amplicons with one nucleotide mismatch. OTUs at $<0.1 \%$ relative abundance are discarded. The quality of the sequencing was analysed by including a mock community sample in each library (Table S6.2). The output OTU table and centroid OTU sequences were used as input for detection of most wanted [391] and novel species. For statistical analysis we used a rarefied OTU table with 2,500 reads per sample, where samples with $<2,500$ reads were excluded.

Pearson correlation coefficients between bacterial communities in inocula (i.e. faecal material of ICU patients) and their respective growth communities were calculated based on OTU-level data using IBM SPSS statistics 23.0.0.2. Shannon diversity, richness and phylogenetic diversity whole tree metrics of bacterial communities were calculated using QIIME [251]. The two-tailed t-test without assuming equal variance was used to investigate if Shannon, richness and wholetree phylogenetic diversity values of bacterial communities differed significantly when grouped according to experimental variables (e.g. growth medium or supplementation of the medium with antibiotics). The t-test used averaged values for 
biological and technical replicates. The QIIME script compare_taxa_summaries.py was used to calculate Pearson correlation coefficients of OTU-level taxa between mock communities and their theoretical composition. Canonical Correspondence Analysis (CCA) as implemented in Canoco 5 [254] was used to investigate which variables could best explain the variation in bacterial composition between bacterial communities. Linear mixed-effect models were fitted by the R package "ImerTest" (https://CRAN.R-project.org/package=ImerTest) [329] in order to analyse how media type and addition of antibiotics affected bacterial composition. As input an adapted OTU table was used in which values were log1p transformed to meet normality assumptions. Furthermore, OTUs were removed from the table if they were detected in $<5$ samples or by $<50$ reads across all samples. Parameter-specific $p$-values were obtained by using the Satterthwaite approximation. P-values were corrected for multiple testing by the function p.adjust in the package "stats", using methods "Bonferroni" and "BH". Bray-Curtis dissimilarity hierarchical clustering was performed using R package 'Vegan' based on OTU-level relative abundance data of bacterial communities.

In order to investigate the presence of most wanted taxa [391] and novel species, the representative reads of the OTUs were compared by BLASTn [283] to the V1-V3 sequence data of the most wanted OTUs, and to the 16S rRNA genes in the SILVA database of type strains [401], respectively. A custom Perl script was used to parse the BLAST results for the best hits (bitscore sorted). Furthermore, the script tabulated the relative abundance of the OTUs and their distribution across all samples.

\section{Targeted cultivation}

Based on analysis of the 16S rRNA gene sequence data of the bacterial growth communities, OTUs were selected for targeted isolation. Therefore, the original faecal inoculum and the enriched growth fractions that contained the target OTU were re-plated under identical conditions, i.e. on PAO chips placed on the same media. A dilution series was inoculated to yield single colonies. Per PAO chip, three colonies per unique colony morphology were transferred to fresh PAO chips. Subsequently, the 16S rRNA gene was amplified using the 27F and 1492R primers [257], and the PCR products were Sanger sequenced at GATC Biotech (Cologne, Germany) using the 907R primer [258]. The 16S rRNA gene sequences were compared by BLASTn to those in the NCBI ribosomal 16S RNA sequences database 
for species identification. Only for target species the near full-length 16S rRNA gene was then Sanger sequenced using the $27 \mathrm{~F}$ and $1492 \mathrm{R}$ primers.

\section{Results}

\section{Bacterial growth on PAO chips}

As inoculum, faecal samples were used from 20 patients that the time of sampling had received SDD treatment for no longer than five days. Three 10-fold serial dilutions of the samples, starting at undiluted, were inoculated in duplicate on PAO chips on top of GAM and CP agar media, either with or without supplementation of the SDD antibiotic cocktail. Agar media inoculated at the lowest dilution of faecal material and in absence of antibiotics always yielded confluent growth on GAM media, whereas on CP media confluent growth was observed on 34 of 40 PAO chips (Table S6.3). In general, less biomass grew on media if the faecal material was inoculated at a higher dilution, if the media contained the SDD cocktail of antibiotics, and if the faecal material was inoculated on CP media. Growth on most PAO chips (371 of 480) was confluent as opposed to colonies that could be visually distinguished (Figure 6.1A). Faecal inocula (in duplicate) and a selection of PAO chips with bacterial growth, including chips inoculated with undiluted and 100-fold diluted faecal suspensions, as well as with 10-fold diluted suspensions for three samples, were analysed by $16 \mathrm{~S}$ rRNA gene amplicon sequencing, which amounted to a total of 324 samples. The NG-tax pipeline was used to process the sequencing data of our samples as well as mock communities with known composition that were added to each library [400]. The average Pearson correlation value of OTU-level taxa between the included mock communities and their theoretical composition was 0.82 (min-max 0.77-0.88), supporting the reliability of the applied approach (data not shown). Samples with 0 reads $(n=4)$ assigned were removed from all further analysis yielding 319 samples with an average read depth of 40,999 \pm 49,592 (s.d.) reads (Table S6.4), and 3,832 assigned OTUs. 

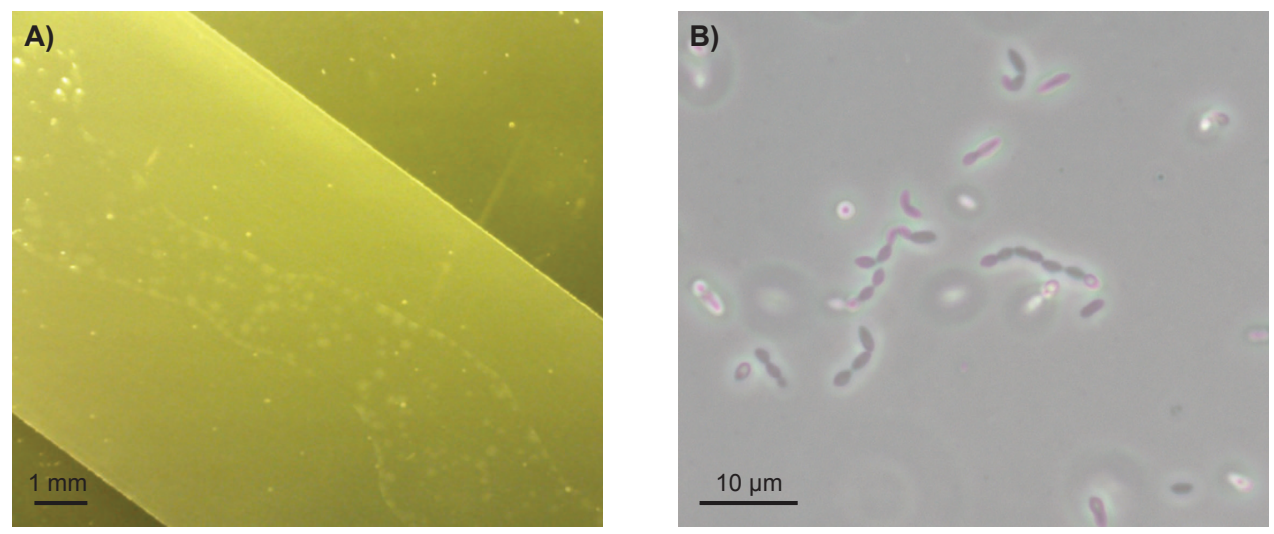

Figure 6.1 A) Close-up photograph of microbial growth on a PAO chip that was placed on top of CP agar. The chip was inoculated with 10X diluted cryo-preserved faecal sample from patient 188 . The area that was inoculated is visualized as a smear in which individual microbial colonies can be distinguished. The white dots in the picture represent air bubbles in the agar medium. B) A light microscopy picture of the strain corresponding to OTU3088 that was isolated by a targeted approach. The strain shares $99 \% 16$ S rRNA gene identity with Sellimonas intestinalis BR72 ${ }^{\top}$.

\section{Comparison of bacterial growth communities}

\section{Bacterial diversity}

Averaged across all faecal samples, the most abundant bacterial phyla were Firmicutes (60.5\% \pm 23.5 [s.d.]), Bacteroidetes (33.9\% \pm 25.3$)$, Proteobacteria $(2.60 \% \pm 6.40)$, Actinobacteria $(1.56 \% \pm 5.00)$ and Verrucomicrobia $(0.49 \% \pm 1.22)$ (Figure 6.2A). The corresponding growth communities on GAM and CP media were dominated by Firmicutes and Bacteroidetes, together comprising on average $>80 \%$ of the bacterial community. On GAM agar without antibiotics the Proteobacteria on average constituted $5.1 \% \pm 16.7$ of the communities whereas on GAM agar with the SDD cocktail (GAM-SDD), proteobacterial relative abundance was $0.033 \% \pm 0.14$. Similarly, on CP-SDD media the Proteobacteria had reduced relative abundance (a decrease from $13.6 \% \pm 27.7$ to $9.8 \% \pm 2.92$ ) as compared to CP media without antibiotics. Notably, Cyanobacteria were not detected in the faecal samples or on GAM media, but they were detected on CP media averaging $0.51 \% \pm 6.40$ relative abundance. As expected, the average Shannon diversity of faecal samples was significantly higher than that of growth communities grouped by medium, addition of antibiotics or dilution (two-tailed t-test, $\mathrm{p}=<0.01$ for all comparisons) (Figure 6.2B). Lower Shannon diversity values were also observed in growth communities inoculated with more diluted faecal sample. However, this difference was only significant between communities on CP-SDD agar that were inoculated with undiluted 
and 100 -fold diluted faecal sample (two-tailed t-test, $p=0.01$ ). The addition of the SDD antibiotics significantly reduced the Shannon diversity on GAM media ( $p=$ $<0.01$ ) but not on CP media. Differences in OTU richness and whole-tree phylogenetic diversity between the sample groups followed the same trends as differences in Shannon diversity i.e. higher values were obtained for faecal samples and lower values were obtained if media were inoculated with more diluted faecal material or included antibiotics (Figure S6.1). However, surprisingly, a higher dilution of the faecal inoculum did not affect whole-tree phylogenetic diversity on CP media without antibiotics $(p=0.81)$. Canonical correspondence analysis (CCA) of OTU-level data from all bacterial communities (faecal inoculates and cultivable fractions) indicated that cultivation medium and presence/absence of antibiotics could explain in total $3.7 \%$ of the variation in bacterial composition ( $p=0.002$ ) (Figure 6.3). However, bacterial growth on CP agar was not found to be significantly affected by the addition of the SDD cocktail of antibiotics. The dilution factor of faecal inocula was also evaluated as explanatory variable but was found to not affect bacterial composition.

A)

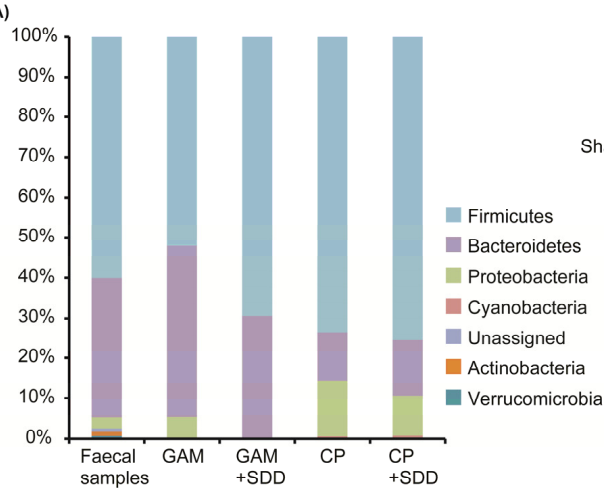

B)

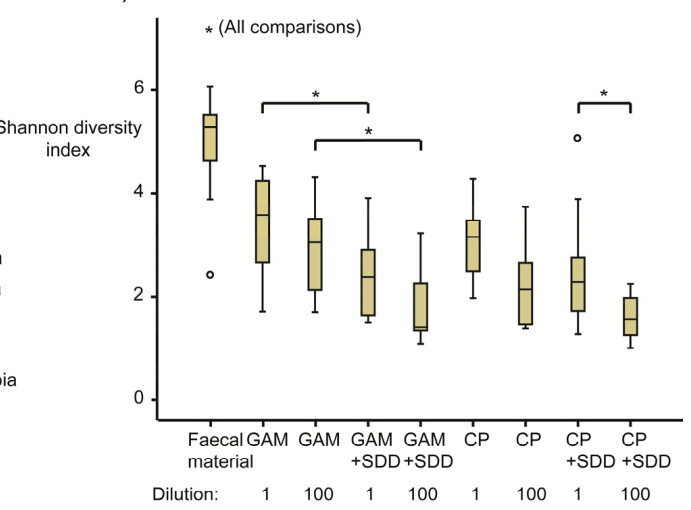

Figure 6.2 A) Bacterial phyla that were detected in the faecal samples of 20 intensive care patients and in their corresponding growth communities on GAM and CP agar media. Growth on these media was further subdivided based on the addition of the SDD antibiotics. Phyla with a relative abundance $<0.5 \%$ are not shown. The relative abundance values are based on the combined reads of all samples in the different experimental groups. B) Boxplots depicting the distribution of Shannon diversity values of bacterial communities in the different experimental groups. Asterisks indicate that Shannon values of bacterial communities in experimental groups were significantly different $(p=<0.05)$ based on the two-tailed t-test. Medium (i.e. GAM vs. CP) did not significantly affect Shannon values of bacterial growth communities. Shannon values of faecal samples were in all cases higher than those for growth communities, irrespective of medium, dilution and addition of antibiotics ( $p=<0.01$ for all comparisons). 


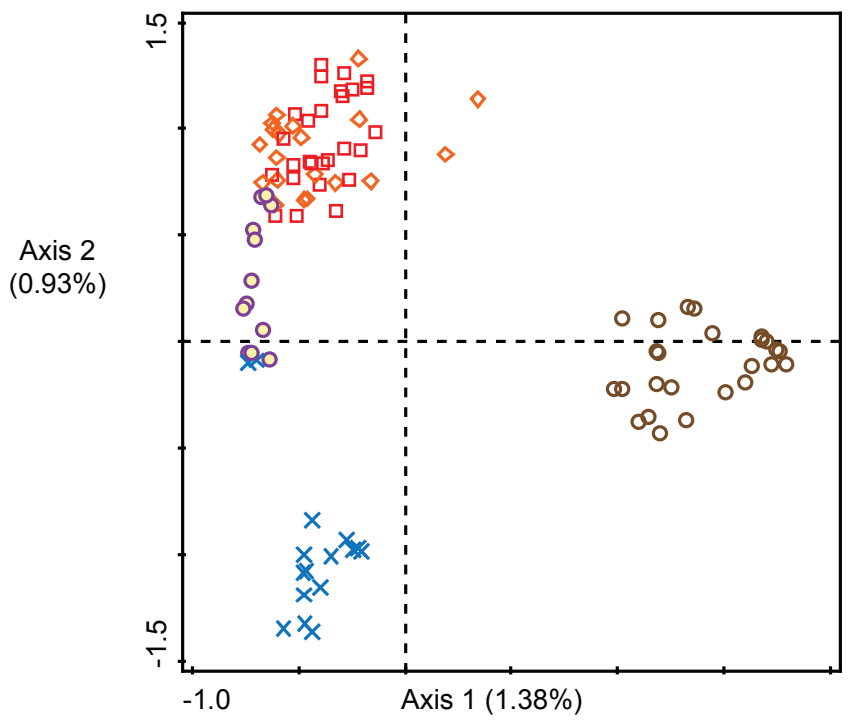

Samples

$$
\begin{array}{lll}
\bigcirc-\text { faeces } & X-\text { GAM agar } & \square-\mathrm{CP} \text { agar } \\
& \bigcirc-\text { GAM agar with the SDD antibiotics } & \triangleright-\mathrm{CP} \text { agar with the SDD antibiotics }
\end{array}
$$

Figure 6.3 Canonical correspondence analysis (CCA) of OTU-level data was used to investigate to what extent growth conditions can explain the variation in the composition of bacterial communities. Included in the analyses were the faecal samples (inocula) as well as their corresponding growth communities on GAM agar and CP agar. Growth on agar media was further distinguished based on the addition of the SDD antibiotics.

\section{Inter-individual differences in communities}

While bacterial communities grouped by experimental variables (i.e. medium or addition of antibiotics) showed significant differences, we were also interested in potential differences between patients. Hierarchical clustering of OTU-level data using Bray-Curtis dissimilarity indicated high dissimilarity between individual faecal samples with mutual dissimilarity values being $>0.8$ in all but two cases (Figure 6.4). Growth communities derived from only 9 of 20 patients all clustered together indicating that other factors besides inoculum influenced bacterial growth. For example, growth communities of patient 131 all clustered together with mutual dissimilarity values $<0.25$ whereas for growth communities of patient 236 dissimilarity values exceeded 0.8 . Moderate clustering by medium and presence/absence of antibiotics confirmed that cultivation conditions affected growth as was shown before by CCA. We also evaluated by Pearson correlation to what extent bacterial growth 
communities resembled the faecal samples from which they were derived (Figure S6.2). For different individuals the average Pearson correlations ranged from 0.02 to 0.99, indicating that there were large inter-individual differences in bacterial cultivability. No trends were discovered between the applied medium, antibiotics and/or dilution of faecal inoculum, and how well the growth communities resembled the faecal samples.

Effects of media composition and antibiotics on bacterial growth

In the following, we aimed to identify OTUs that were enriched as a result of specific cultivation conditions. We fitted linear mixed-effect models on OTU-level data so that also differences between individual patients could be taken into account (Table S6.5). A total of 35 OTUs were significantly enriched under the different cultivation conditions (Table 6.1). Considering Bonferroni-corrected p-values, a total of seven OTUs belonging to the families Bacteroidaceae (5 OTUs), Staphylococcaceae (1 OTU) and Enterococcaceae (1 OTU) were present in significantly higher abundance on GAM media as compared to the respective faecal samples. In contrast, on CP media, OTUs belonging to the families Halomonadaceae (2 OTUs), Lachnospiraceae (6 OTUs), Ruminococcaceae (1 OTU), Streptococcaceae (1 OTU), Enterococcaceae (3 OTUs), Porphyromonadaceae (2 OTUs), and Oxalobacteraceae (1 OTU) were enriched. A significantly lower abundance of Ruminococcaceae spp (1 OTU). Enterobacteriaceae spp (6 OTUs) and Lachnospiraceae spp. (4 OTUs) was detected on media supplemented with the SDD antibiotics in comparison to media without antibiotics, indicating that the antibiotics inhibited the growth of these bacteria. 


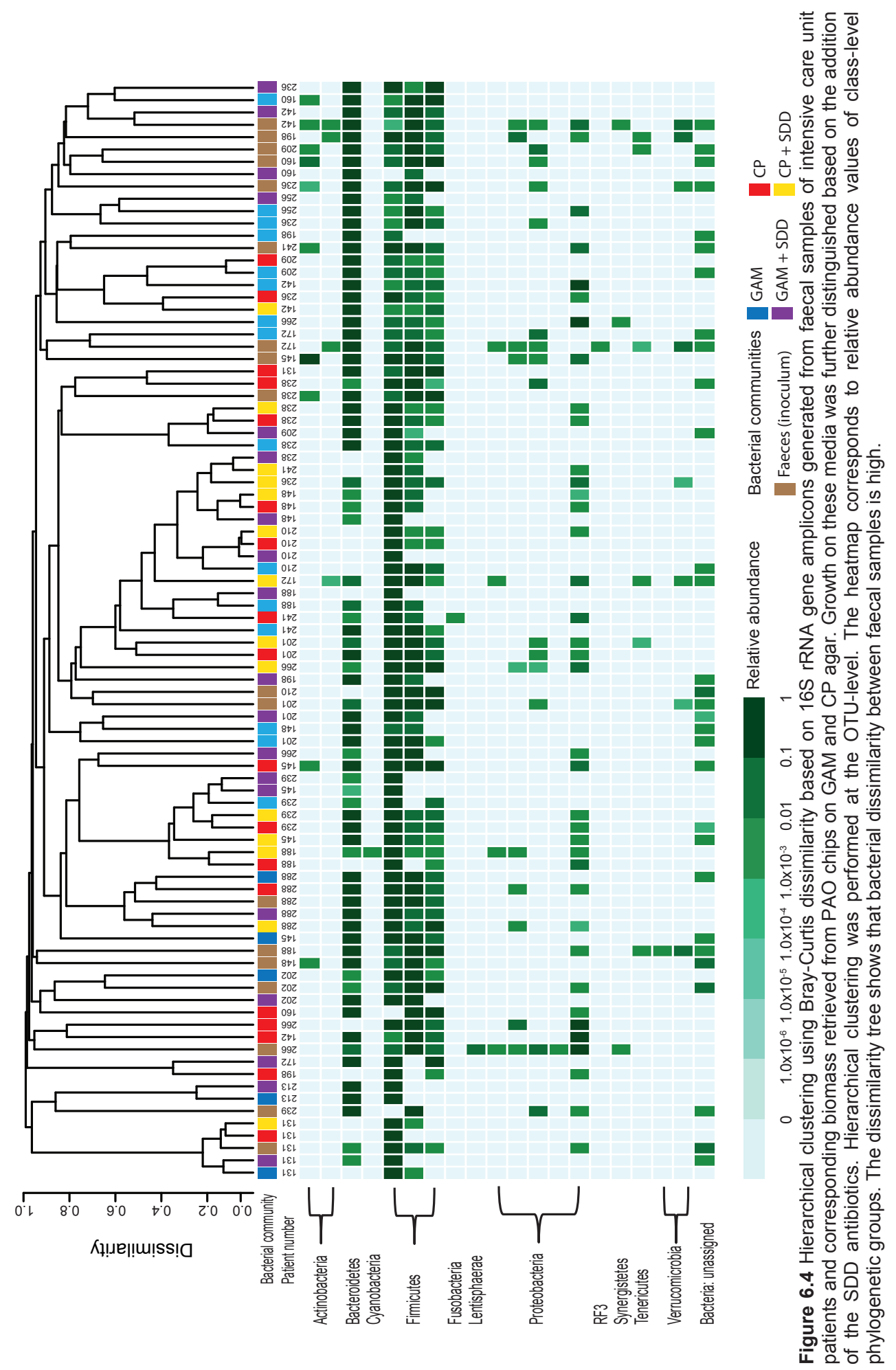


Table 6.1 Linear mixed-effect models of OTU-level composition data of bacterial growth were applied to investigate which OTUs varied in abundance as a results of cultivation conditions. This table lists the taxonomic affiliations of OTUs that were found to be enriched at Bonferroni corrected $p$-values of $<0.05$. Bacterial communities were cultivated on GAM agar and CP agar, both in the presence and absence of the SDD antibiotics.

\begin{tabular}{|c|c|c|c|}
\hline & & Taxonomy & \\
\hline Enriched on & No. of OTUs & Family & Genus \\
\hline \multirow[t]{3}{*}{ GAM agar } & 5 & Bacteroidaceae & Bacteroides \\
\hline & 1 & Staphylococcaceae & Staphylococcus \\
\hline & 1 & Enterococcaceae & Enterococcus \\
\hline \multirow[t]{7}{*}{ CP agar } & 2 & Halomonadaceae & Halomonas \\
\hline & 6 & Lachnospiraceae & Unspecified \\
\hline & 1 & Ruminococcaceae & Unspecified \\
\hline & 1 & Streptococcaceae & Streptococcus \\
\hline & 3 & Enterococcaceae & Enterococcus \\
\hline & 2 & Porphyromonadaceae & Parabacteroides \\
\hline & 1 & Oxalobacteraceae & Undibacterium \\
\hline \multirow[t]{3}{*}{ Media (CP and GAM) without antibiotics } & 1 & Ruminococcaceae & Unspecified \\
\hline & 6 & Enterobacteriaceae & Escherichia-Shigella \\
\hline & 4 & Lachnospiraceae & Unspecified \\
\hline CP agar without SDD cocktail of antibiotics & 1 & Ruminococcaceae & Unspecified \\
\hline
\end{tabular}

\section{Growth of novel species}

We further aimed to investigate whether novel species or members of taxa on the most wanted list were present in the cultivable fraction of the faecal samples. Eleven high priority most wanted OTUs were detected in the growth communities; however, none of these OTUs was present at a relative abundance of $>0.8 \%$ (Table S6.6). Furthermore, three medium priority most wanted OTUs (OTUs 236, 172 and 288) were detected in the cultivable fraction with 1-3\% relative abundance. Nevertheless, members of medium priority OTUs were not considered candidates for isolation because they shared $100 \%$ identity with strains that were previously isolated. Comparison of OTUs with the SILVA database of type strains yielded 16 OTUs with $>2 \%$ relative abundance of which the OTU representative read shared $<95 \%$ identity with the 16S rRNA gene sequence of the closest type strain (Table 6.2). Therefore, these OTUs were considered to i) potentially represent novel species, and ii) to be sufficiently abundant for isolation by colony picking. Among these 16 OTUs, OTUs 3088, 322, 3797, 2642 and 2024 were considered prime candidates for targeted isolation based on their high relative abundance on the PAO chips $(>5 \%)$ and novelty. OTU3088 shared $93.4 \%$ identity with the closest type strain, that is, Ruminococcus torques, and was present on GAM-SDD agar at $49.8 \%$ relative 
abundance (Table S6.6). OTU3088 was also detected on GAM, CP and CP-SDD agar media, albeit at a lower relative abundance. We detected three additional OTUs (OTUs 3067,3103 and 3070 ) at $>2 \%$ relative abundance of which the closest type strain was also Ruminococcus torques. Since these three OTUs were always detected in samples that contained OTU3088, and since their representative reads shared high nucleotide identity with OTU3088 (>99\%), we considered that they may be derived from the same bacterial strains. The best hits in the SILVA type strain database of strains OTU322 and OTU3797 were Hydrogenoanaerobacterium saccharovorans and the chloroplast of the diatom Thalassiosira pseudonana, respectively, and both were detected at $>10 \%$ relative abundance (OTU322, max. 23.9\%; OTU3797, max. 13.2\%). Both OTU322 and OTU3797 were only detected at $>1 \%$ relative abundance on CP agar media in the absence of the SDD antibiotics. The representative read of OTU2642 shared $94.7 \%$ nucleotide identity with the 16 S rRNA gene of Bacteroides ovatus. OTU2642 was only detected on GAM media at a maximum of $7.4 \%$ relative abundance. Finally, the closest type strain of OTU2024 was Oscillibacter ruminantium, and it was detected at $>1 \%$ relative abundance exclusively on CP-SDD media.

\section{Targeted cultivation}

To provide proof of concept, we aimed to isolate strains corresponding to OTUs 3088 and 2024 as they were considered prime candidates for isolation based on novelty. Furthermore, the fact that these OTUs grew on media containing the SDD antibiotics suggested they may be antibiotic resistance reservoir species. We prepared dilution series of the growth fractions in which these OTUs were most enriched and subsequently inoculated the diluted samples under the exact conditions that previously yielded enrichment of the target OTUs. This experiment, however, did not yield isolation of the target OTUs. Therefore, the protocol was repeated using the original faecal samples as inocula instead of the enriched growth fractions. By this method we isolated a member of OTU3088, which was demonstrated by the fact that the representative read of OTU3088 shared $100 \%$ identity with the $16 \mathrm{~S}$ rRNA gene Sanger read of our isolate (Figure 6.1B). BLASTn of the 16S rRNA gene read of the isolate against the NCBI ribosomal 16S RNA sequences database showed that closely related strains (98-99\% nucleotide identity) have recently been isolated in four other laboratories. One closely related strain that was isolated from human faeces 
was recently published as a novel species named Sellimonas intestinalis BR72 [402].

\section{Discussion}

In this investigation, we studied the cultivability of anaerobic human faecal bacteria in order to isolate strains that can serve as reservoirs of antibiotic resistance. Therefore, bacterial growth communities on GAM and CP agar media derived from faeces of 20 ICU patients receiving SDD therapy were studied. We applied the MicroDish PAO chip to reduce potential toxicity of agar and to facilitate efficient parallel processing of a large number of samples. We also applied media supplemented with the SDD antibiotics that the patients received upon arrival on the ICU. A first selection of bacteria was made by comparing the cultivated species with the strains on the most wanted list that comprises human-associated bacteria of which the genome has not yet been sequenced and that are grouped into priority classes based on novelty [391]. We did not detect high priority taxa at $>1 \%$ relative abundance in the growth communities. Medium priority taxa were detected at $1-3 \%$ relative abundance but they shared $100 \%$ 16S rRNA gene sequence identity with strains for which the genome has been already sequenced. Therefore, due to the low relative abundance and/or high similarity to previously genome-sequenced bacteria, the detected medium and high priority taxa were not further considered prime candidates for isolation. However, comparison of the OTU centroid reads with the strains in the SILVA type strain database yielded 16 OTUs with $<95 \%$ nucleotide identity and $>2 \%$ relative abundance. Based on their novelty, members of these OTUs were considered candidates for isolation. Although their prevalence in the gut microbiota is expected to be $<20 \%$, i.e. they are not on the most wanted list, understanding the biology of such populations might be highly relevant in a more personalized approach, where individual-specific microbiota signatures are considered key to success [403]. Our results show that novel human-associated bacteria can still be cultivated using conventional methods. The extent to which novel bacteria can still be isolated by conventional methods was shown recently by Browne and co-authors in an experiment in which they isolated $\sim 4,000$ pure culture bacterial strains from faeces of six human individuals. The authors found that these isolates comprised as much as $96 \%$ of the bacterial abundance at the genus level and $90 \%$ of the bacterial 


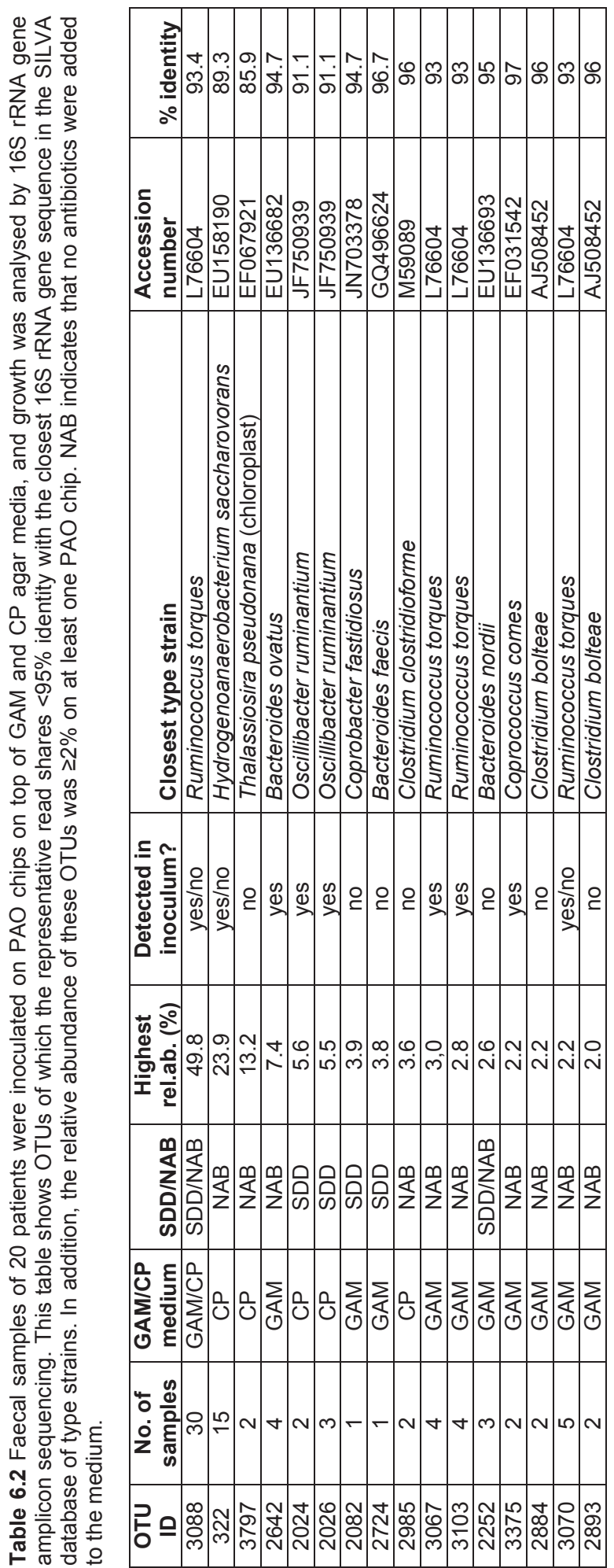


abundance at the species level based on average relative abundance across faecal samples of six individuals [86].

Almost all of the 16 OTUs detected in the growth communities represented novel species that belonged to the Firmicutes (11 OTUs) and Bacteroidetes (4 OTUs), and these phyla were also detected in highest relative abundance in the faecal samples. Notably, a single OTU was detected on CP agar at a maximum relative abundance of $13.2 \%$ that shared $85.9 \%$ nucleotide identity with the best hit in the SILVA type strain database, namely the chloroplast of Thalassiosira pseudonana. Chloroplasts are thought to have originated from cyanobacteria [404], and this finding might suggest the growth of a eukaryote capable of photosynthesis. OTU3088, which is a novel OTU belonging to the Firmicutes, was detected in samples of nine different patients. Furthermore, on one PAO chip OTU3088 was detected in the presence of the SDD antibiotics at a relative abundance of $49.8 \%$. Therefore, it was selected for isolation, which was achieved on GAM-SDD media (Figure 6.1B). After isolation, the 16S rRNA gene of the isolate turned out to share $99 \%$ nucleotide identity with the recently published Sellimonas intestinalis [402], and high identity (98-99\%) with three other strains recently isolated from human gut microbiota (accessions KT156811, LN828944 and AY960564). Therefore, even though a close relative of our isolate has been recently described, these results demonstrate that high-throughput cultivationbased screening can be used to isolate novel antibiotic resistant bacteria by a targeted approach. The strain in question is a candidate to be further analysed as resistance reservoir (e.g. by genome sequencing). Besides OTU3088, five other novel OTUs were found to grow in the presence of the SDD antibiotic cocktail, and as such are additional candidates for isolation and characterization (Table 6.2). However, it should be noted that antibiotics may be broken down by adjacent bacteria, and that therefore these bacteria may themselves be not resistant. For example, cefotaxime may be degraded through the secretion of a $\beta$-lactamase [405, 406]. Bacterial isolation in general can also be hampered by the dependence on microbe-microbe interactions or host-microbe interactions [87]. Out of the 16 novel OTUs detected on agar media, only OTU (OTU3088) was detected on both GAM and $\mathrm{CP}$ media at $>2 \%$ relative abundance. This indicates that the number of target species for isolation might be increased by including different media. 
We also investigated bacterial growth not pertinent to the isolation of novel species. We showed that the composition of bacterial growth communities was significantly impacted by medium and by supplementation of media with antibiotics (Figure 6.3). The cocktail used in SDD therapy contains antibiotics that are predominantly active against Gram-negative bacteria and fungi [407], and was designed in order to eradicate potentially pathogenic bacteria from the gut without harming the anaerobic microbiota [392, 396]. Indeed, we found that six OTUs belonging to the genus Escherichia, one of the SDD-target taxa, grew in significantly lower relative abundance on media containing the SDD cocktail. However, we also found that five OTUs belonging to the families Ruminococcaceae and Lachnospiraceae grew to a significantly lower relative abundance in the presence of the SDD antibiotics. Members from these families are Gram-positive and lack aerobic respiration [408], and are therefore collaterally affected by the application of the SDD antibiotics that aim to lower the risk of infection with Gram-negative aerobic opportunistic pathogens [392]. Remarkably, we found that the correlation of bacterial composition of the faecal communities and the growth communities varied extensively between patients. We cannot exclude that this may have resulted from differences in viability of cryopreserved faecal samples, but it might also be related to the individual-specific composition of the gut microbiota.

In conclusion, we have shown that high-throughput screening of growth communities for bacterial resistance can guide targeted isolation of potential reservoir species. The fact that a member of one novel antibiotic-resistant OTU (OTU3088) was successfully isolated demonstrates the viability of the approach. Follow-up isolation and characterization will be required to analyse the role of previously uncultivated species in the dissemination of resistance genes in the gut microbiota, including the transfer to potential pathogens.

\section{Acknowledgements}

This work was supported by the European Union through the EvoTAR project (Grant agreement no. 282004). We thank Edoardo Saccenti for advice on statistical analyses. We thank the ICU staff and the Department of Medical Microbiology (contact person: Willem van Schaik) at the University Medical Center Utrecht for collecting and preserving the faecal samples of ICU patients. 


\section{Supplementary information}

\section{Supplementary Tables and Figures}

Tables S6.1, S6.2, S6.3, S6.4, S6.5 and \$6.6 are available on request.

A)

B)
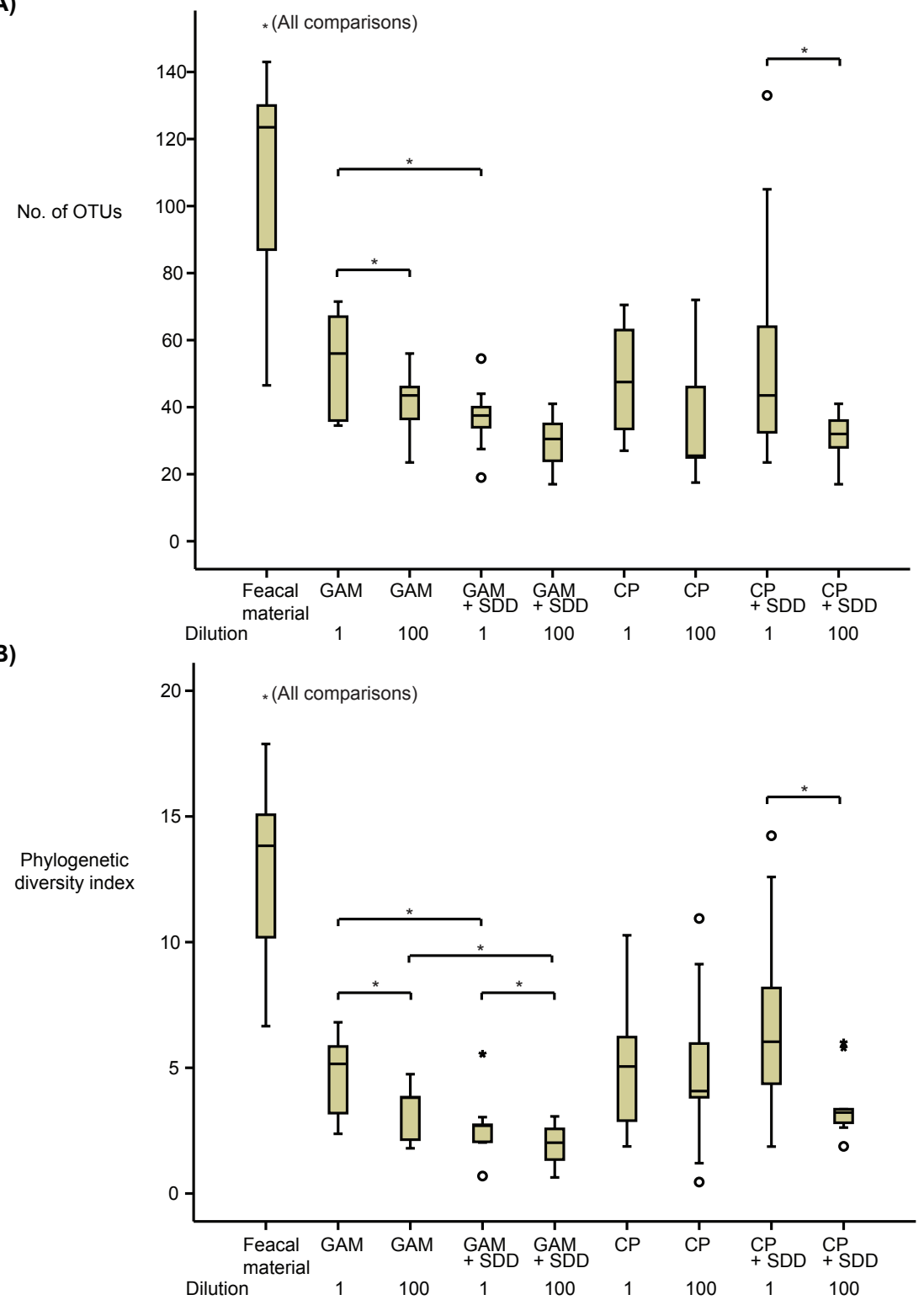

Figure S6.1 Boxplots depicting the distribution of no. of OTUs (panel A) and phylogenetic diversity values (Panel B) of bacterial communities in different experimental groups. The experimental groups are the faecal inocula and their corresponding growth communities on GAM and CP agar media. Growth on these media was further subdivided based on the addition of the SDD antibiotics. Asterisks indicate that these richness and diversity values of bacterial communities in experimental groups were significantly different $(p=<0.05)$ based on the two-tailed t-test. 

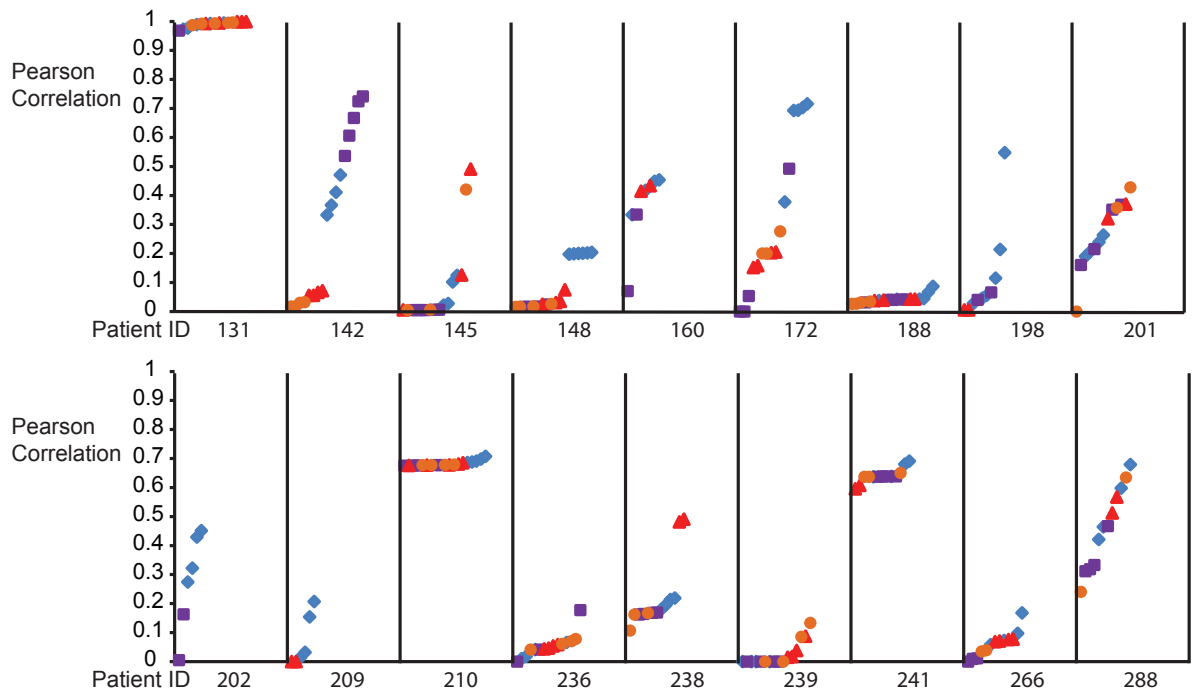

Data points represent biomass growing on:

- GAM agar

- GAM agar with the SDD antibiotics

- CP agar

- CP agar with the SDD antibiotics

Figure S6.2 The bacterial communities in the faecal samples of 18 intensive care patients were compared to the corresponding growth communities on GAM agar and CP agar by calculating the Pearson correlation coefficients of OTU-level taxa. Growth on these media was further distinguished based on the addition of the SDD antibiotics. Per patient the Pearson coefficients are sorted from lower to higher values. 

General Discussion 
Application of antibiotics has resulted in enormous societal benefits in terms of improved patient treatment outcomes and more efficient livestock production. Over time, however, high antibiotic use contributed to the evolution of antibiotic resistance in human pathogens [9], thereby negatively affecting patient treatment outcomes. In fact, "superbugs" have emerged that are resistant to virtually all antibiotics, including the drugs of last resort [409, 410]. As a result, numerous scientists now believe that we are heading towards a post-antibiotic era in which mortality due to infections will rise [411-413]. One way by which microorganisms can become resistant is via acquisition of resistance-conferring genes from other microorganisms in the environment. However, surprisingly little is yet known about the mechanisms and frequencies of these resistance dissemination events, even though knowledge regarding these topics is crucial to combat antibiotic resistance [40, 45].

The main aim of this thesis was to identify resistance reservoirs, which can be individual bacterial species or microbial communities in specific environments, and to subsequently characterize the resistance determinants and their dissemination in the microbial biosphere. Particular focus was given to sponge-associated bacteria and to anaerobic bacteria from the human gut microbiota. Sponges often contain diverse microbial communities that are rich sources of antimicrobial compounds [118, 277]. This notion, together with the fact that the sponge microbiota has not yet been methodically studied in the context of antibiotic resistance, triggered us to investigate their potential role as a reservoir of resistance-conferring genes. Among the microorganisms associated with marine sponges, members of the genus Pseudovibrio were studied in more detail by comparative genomics. Pseudovibrio spp. represent a metabolically versatile group of marine microorganisms that potentially play a role as sponge symbionts and hubs of antibiotic resistance. While many studies have focused on antibiotic resistance in the human gut microbiota, relatively few studies specifically reported on anaerobic uncultivated gut bacteria as a reservoir of resistance. To this end, innovative cultivation approaches were applied together with state of the art molecular profiling of microbial composition in order to identify and better understand the contribution of the anaerobic gut microbiota to the antibiotic resistance problem. Finally, this thesis for the first time applied metatranscriptomics to study to resistance gene expression in different biological niches under natural conditions. Figure 7.1 provides a schematic overview of the research described in this thesis. 


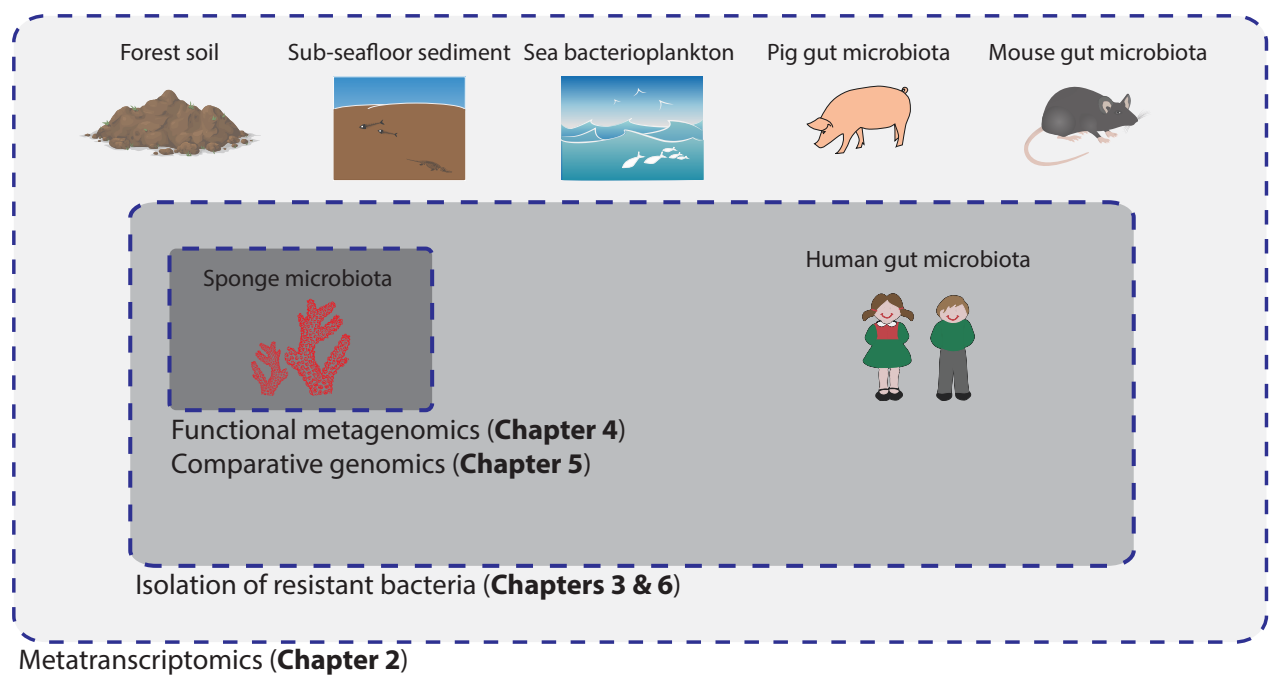

Figure 7.1 Schematic representation of the research described in this thesis. Dashed lines group biological niches that were investigated with particular methods.

\section{Resistance gene expression detected in all investigated niches}

Multiple studies analysing metagenomic data have established that antibiotic resistance genes are present in most, if not all, biological niches that harbour complex microbial communities [69, 97]. In Chapter 2, we went one step further and showed that resistance genes are not only ubiquitously present, but also being expressed in the microbial biosphere. Resistance gene expression was detected in bacteria associated with humans, mice, sea bacterioplankton, a marine sponge, forest soil and sub-seafloor sediment even though these bacteria were unperturbed by anthropogenic antibiotic pressure. In human gut metatranscriptomes and metagenomes studied so far, the detected resistance genes broadly belonged to the same classes, namely tetracycline, macrolide and $\beta$-lactam resistance genes [104, 202]. Interestingly, in gut metagenome data of 162 human individuals the number of detected resistance genes ranged from 33 to 89 [202], whereas in our metatranscriptome data of four human individuals this number ranged from 5 to 20 . Furthermore, quinolone resistance genes were detected exclusively in the metagenome data, albeit at low relative abundance. The lower resistance gene richness in metatranscriptome data cannot be explained by differences in read depth since the metatranscriptome dataset averaged $\sim 6$ million non-rRNA reads and the metagenome datasets averaged $\sim 5$ million reads. As such, these results would 
suggest that a considerable number of resistance genes is present while not being expressed.

However, caution should be taken before concluding that absence of resistance genes in metatranscriptome data also equals absence of expression. In fact, Haas and co-authors found that as much as 5-10 million non-rRNA reads are required to detect all but the rarest transcripts of a single bacterium [414]. Considering that the human gut has been estimated to contain 100-1,000 bacterial species [415-417], it is expected that our datasets of $\sim 6$ million non-rRNA reads do not even approach the required sequencing depth to capture most transcripts. A case in point is the forest soil sample in our study from which both metagenome and metatranscriptome datasets of $\sim 1$ million reads were available. In the soil metatranscriptome dataset macrolide resistance genes were detected whereas in the metagenome dataset chloramphenicol, aminoglycoside, tetracycline, and $\beta$-lactam resistance genes were detected (data not shown). A suitable sequencing depth can be selected based on knowledge about relative abundances of resident bacteria [418]. Clearly, routinely achieved current metatranscriptome sequencing depths, and to a lesser degree metagenome sequencing depths, are not sufficient to fully discern the functionality and activity of low-abundance bacteria in microbial communities. Therefore, while these technical considerations do not undermine the validity of our conclusions based on distinct inter-individual and inter-niche differences in resistance transcripts, it should be considered that observations are limited by an inherent detection threshold.

To validate the hypothesis that resistance genes are often present in microbial communities without being expressed, it is necessary to at the same time analyse the metatranscriptome and metagenome of a sample. The study can include communities that have been subjected to antibiotics to study induction of expression by antibiotics (sample pre- and post-administration of antibiotics). A similar approach can be used to study to what extent other factors such as heat, dietary fibers, solvents or non-relevant antibiotics affect resistance gene expression levels. The inadequate read depth of traditional metatranscriptome sequencing can be circumvented by using a more targeted approach. For example, quantitative PCR can be used to detect the presence or expression of specific resistance genes [78, 419]. Furthermore, RNA CaptureSeq may be applied to achieve unprecedented depth in mining of resistance transcripts [420]. Using this approach, RNA is first transcribed 
into cDNA, after which the cDNA pool is enriched by hybridizing it to arrays that contain probes targeting sequences of interest (i.e. resistance gene sequences). Finally, the cDNA pool is deep-sequenced. It should be noted that a targeted approach can only work if there is prior knowledge on the resistance genes that can be present or expressed in a given biological niche. However, at this time, comprehensive information about resistance gene presence is only available for human gut microbiota.

It has been proposed that eventually antibiotic therapy will be based on resistance potentials of gut microbiota of individuals, as determined by metagenome sequencing [104]. Therefore, clinicians need to first screen patient-associated bacteria for resistance, after which an antibiotic therapy can be tailored to fit the patient's resistome [421]. In our metatranscriptome study, we showed that there are substantial inter-individual differences in the profiles of expressed antibiotic resistance genes. Information about resistance gene expression could provide deeper insights into the immediate resistance potential of bacteria associated with patients suffering from bacterial infections, and as such may be relevant in clinical practice as well. Such individualized treatments could be used to treat non-urgent infections if meta-level resistome sequencing and downstream data analysis can be accomplished in a short time frame [422].

\section{Sponges as a reservoir for antibiotic resistance genes}

Chapter $\mathbf{3}$ and Chapter 4 covered the investigation of antibiotic resistance in sponge microbiota. It was hypothesized that sponges are an important resistance reservoir because they often contain diverse and complex microbial communities that can produce bioactive compounds, including those with antimicrobial activity. The main aim was to identify antibiotic resistant bacteria and their resistance genes and associated mobile elements, in order to evaluate to what extent sponges may contribute to emerging resistance. In addition, knowledge about identified resistance genes could contribute to the development of compounds that inhibit resistance mechanisms [423, 424], and could lead to the discovery of novel antibiotic biosynthesis clusters that are frequently co-localized with resistance genes in bacterial genomes [425]. In Chapter 3, we described the isolation of 31 different bacterial strains from the sponges $A$. aerophoba, $P$. ficiformis, and $C$. candelabrum on agar media supplemented with antibiotics. Subsequently, application of functional 
metagenomics, using pooled genomic DNA of the isolates as starting material, yielded the identification of functional resistance genes in 15 of these 31 bacteria (Chapter 4). In the combined samples of said sponges, 972 OTUs were detected based on clustering of $16 \mathrm{~S}$ rRNA gene amplicon sequences at $97 \%$ identity; however, none of these OTUs corresponded to the 31 bacteria that were obtained in pure culture. This failure to recover OTUs may in part be explained by selective growth due to the supplementation of the cultivation media with antibiotics. Since our cultured isolates only comprised a small fraction of bacterial sponge residents, we decided to also interrogate the sponges culture-independently for the presence of functional resistance genes. The metagenomic libraries, which were prepared starting with DNA from sponge tissue, yielded only seven unique resistance genes even though the cumulative sponge DNA-derived libraries were $2.7 \mathrm{Gbp}$ in size. The fraction of clones with eukaryotic DNA in the sponge DNA-derived libraries was not investigated, and as such we cannot decidedly link the low number of resistant clones to the presence of eukaryotic DNA in these libraries. In our sponge DNA-derived libraries we recovered none of the resistance genes that were found in our isolate-derived libraries, even though both originated from the same sponge samples. Therefore, these results imply that resistance gene presence in these sponges is vastly underestimated. Previously, it has been shown that expanding the metagenomic library size for a given biological niche will increase the number of unique resistance genes that are retrieved [69, 426-428]. Given that $>8,500$ sponge species are currently listed in the World Porifera Database [429], and given that sponge-associated microbial communities are largely sponge-specific [126-128], it may be anticipated that our research only offered a first glimpse of an enormous resistance gene diversity amongst sponges. The resistance genes that we identified were limited to those that could be heterologously expressed in E. coli. Heterologous expression can be impeded by differences between bacterial species in cellular machinery including promotor recognition, recognition of the ribosome binding site and translation of rare codons [427, 430]. Identification of resistance genes was further limited by $E$. coli being intrinsically resistant to several antibiotics, and in particular to those that target Gram-positive bacteria [431]. To overcome these limitations, an alternative surrogate host may be used such as Bacillus subtilis [432] or Lactococcus lactis [433]. For resistant bacteria in pure culture, transposon- 
insertion sequencing methods may be used to identify genomic loci that affect sensitivity to antibiotics [434].

It was remarkable that 11 of our sponge isolates harboured functional resistance genes against gentamicin, chloramphenicol, rifampicin, ampicillin and/or trimethoprim while the strains themselves were not resistant to these antibiotics in the phenotypic tests described in Chapter 3. One of the possible explanations, as speculated in Chapter 3, might be that E. coli cells become resistant due to the high copy number of the resistance-gene-harbouring plasmids, thereby increasing the concentration of active enzyme. In turn, in the sponge isolate the resistance gene, if encoded chromosomally, may be present as a single copy, which might generate insufficient active enzyme to provide antibiotic resistance to the host. Still, it should also be considered that for our sponge isolates MIC values that correspond to the upper limit of the wild-type population of a particular species have not been defined. Typically, these epidemiological cut-off values are well-defined for human pathogens but not defined for environmental bacteria [435]. Therefore, it may be that the identified resistance genes confer resistance to the original host in its natural environment but not at the (artificially high) antibiotic concentration that was used during resistance profiling. This phenomenon could especially play a role because the antibiotic testing concentrations were set relatively high compared to epidemiological cut-off values of common pathogens aiming to reduce the isolation of bacteria that are intrinsically resistant. To select an antibiotic testing concentration, we used the cut-off values from four bacterial taxa (Enterobacteriaceae, $P$. aeruginosa, Pseudomonas spp. and Staphylococcus spp.) from the EUCAST and CLSI databases [436-438]. The highest antibiotic concentration cut-off for "resistance" amongst these four taxa was multiplied by 1.5 , rounded off, and subsequently used as testing concentration. For example, for cefotaxime the available epidemiological cut-off values were $2 \mu \mathrm{g} / \mathrm{ml}$ (Enterobacteriaceae, CLSI), 4 $\mu \mathrm{g} / \mathrm{ml}$ (Enterobacteriaceae, EUCAST), $32 \mu \mathrm{g} / \mathrm{ml}$ ( $P$. aeruginosa, CLSI) and $64 \mu \mathrm{g} / \mathrm{ml}$ (Staphylococcus spp., CLSI). The highest value (i.e. $64 \mu \mathrm{g} / \mathrm{ml}$ ) was multiplied by 1.5 , and subsequently rounded off to yield a testing concentration of $100 \mu \mathrm{g} / \mathrm{ml}$.

It is interesting to note that the functional resistance genes from spongeassociated microbiota identified in our study had only limited similarity to resistance genes found in human pathogens, or even in human-associated bacteria. This finding was supported by an experiment that used qPCR to detect antibiotic resistance 
genes in DNA samples from the three sponges: $A$. aerophoba, $P$. ficiformis and $C$. candelabrum. The samples were interrogated with respect to 84 resistance genes that are frequently present in the human gut microbiota including those providing resistance to aminoglycosides, bacteriocins, $\beta$-lactams, macrolides, fosfomycin, linezolid, quinolones, tetracycline and vancomycin [396]. None of these clinically relevant resistance genes were detected in the sponge samples by qPCR, and comparison of the qPCR primers with the resistance genes identified by functional metagenomics confirmed that the primers indeed did not sufficiently match with these sequences to allow for PCR amplification (data not shown). On the other hand, a recent study detected the mecA, mupA, qnrB and tetL genes in the sponge Petromica citrina by PCR with gene-specific primers [142]. It is not unexpected that clinically relevant resistance genes are rarely detected in the environment as the environmental origin of these genes has only been well-established for CTX-M $\beta$ lactamases and qnrA [111-113]. For example, bla KLUA from a member of the genus Kluyvera, which are environmental organisms [439], was found to be highly similar to various clinically relevant plasmid-encoded CTX-M-type $\beta$-lactamases. Mobilization of bla KLUA was expected to be probable due to its association with a mobile element, and the fact that its surrounding sequences shared high identity with those surrounding the clinically relevant CTX-M $\beta$-lactamases $[111,440]$. The fact that clinically relevant resistance genes are rarely detected in other environments is also consistent with the notion that different ecological niches contain a core resistome that overlaps with resistomes of other niches only to a limited extent [99, 104, 105]. Nevertheless, it is clear from functional metagenomics studies that "niche-specific" resistance genes can also function outside their natural environments (i.e. in the laboratory in E. coli) [79]. Therefore, it has been speculated that mobility, rather than functionality, might be the main obstacle preventing resistance gene dissemination [99]. It appears that resistance genes typically need to be associated with mobile elements before disseminating into the microbial biosphere. Amongst our genes identified by functional metagenomics, only 1 of 37 identified genes was associated with a mobile element. We also found that resistance genes identified in libraries prepared from DNA isolated from sponge tissue were less similar to deposited resistance genes than genes identified in libraries prepared from pooled DNA from bacterial isolates. This is consistent with a previous functional metagenomics study showing that resistance genes are more distant from known resistance genes in 
libraries independent of cultivation [185], most likely reflecting differences in cellular machineries between cultivable taxa and yet uncultivated taxa.

Even though the resistance genes that were identified by functional metagenomics showed limited similarity to resistance genes found in pathogens, we did find evidence for resistance gene dissemination between sponges and the microbial biosphere. More specifically, a rifampicin resistance gene and an ampicillin resistance gene were identified in a Bacillus sp. sponge isolate, and both genes shared $>99 \%$ nucleotide identity with those encoded on genomes of various Bacillus megaterium strains. Bacillus megaterium can be found in a wide range of habitats such as soil, sea water, sediment, fish and dried food [441]. These findings complement already existing evidence for resistance dissemination between sponges and the environment that came from the detection of a tetracycline resistance gene in a sponge-associated Bacillus sp. as well as in Bacillus spp. associated with cheese, soil and honey bees [139]. Taken together, these results indicate that resistance genes can indeed move between sponges and the (non-marine) environment. Considering that Bacillus spp. are not sponge-specific bacteria [442], it could be argued that the resistance genes are merely conserved in Bacillus, and that these genes are only transiently present in the filter-feeding sponge. Still, bacteria that are transiently present in sponges may serve as vehicles for the dissemination of sponge-specific resistance genes into the environment. The fact that we identified a novel class of $\beta$-lactamases exclusive to putative sponge symbiotic Pseudovibrio spp. suggests that resistance genes exist that evolved in sponges. To what extent these "sponge-specific" $\beta$-lactamases complement those of other classes in terms of substrate specificity will require additional experiments that compare functionality.

In summary, we can conclude that sponges indeed serve as a reservoir for antibiotic resistance genes that potentially can be transferred to human pathogens. Even though we have not shown direct transfer of an antibiotic resistance gene from a sponge bacterium to a human pathogen, we provided evidence that resistance genes should indeed be able to move between sponges and the greater non-marine biosphere. Furthermore, 15 novel resistance genes ( $<90 \%$ amino acid identity to known genes products) were detected in sponge bacteria indicating that these might contribute to the current spectrum of resistance genes that can potentially be harnessed by human pathogens. Considering that resistance genes in sponge bacteria are rarely associated with mobile elements, and considering the remote 
localization of sponges, it is expected that sponge-associated resistance determinants only pose a very limited immediate risk to human health [435]. Nevertheless, sponges filter large amounts of water [443], and as such the identified resistance genes could be derived from marine-specific microorganisms instead of sponge-specific, and as such might be closer to humans than anticipated. It might be interesting to also explicitly focus on taxa that are considered sponge-specific in future studies [444]. To obtain a more exhaustive account of sponges as a resistance reservoir, more sponges will need to be examined with respect to resistance gene presence. Tracking of resistance genes at the level of individual bacterial strains and individual plasmids will be required to obtain an all-inclusive view of their (potential) clinical impact [445]. This will require a better understanding of population biology of microorganisms, and extensive access to modern molecular techniques (e.g. metagenome sequencing). Quantitative knowledge about HGT rates of resistance genes under different conditions would be required to identify factors that contribute to the emergence of resistance.

\section{The genus Pseudovibrio as a resistance reservoir}

The hypothesis that Pseudovibrio spp. form a symbiotic relationship with marine invertebrates, and especially sponges, is well-supported. Firstly, cultivation studies sampling both sponges and the surrounding sea water only yielded Pseudovibrio isolates from sponge material $[149,150]$. Secondly, research described in Chapter 5 of this thesis and previous studies have identified symbiosis-related genetic elements in genomes of Pseudovibrio spp. [151, 304]. Lastly, by analysing publicly available metagenomes (Chapter 5), it was found that the relative abundance of Pseudovibrio spp. in sea squirt and sponge $16 \mathrm{~S}$ rRNA gene datasets $(>5 \%)$ was substantially higher than in sea water and aquatic 16S rRNA gene datasets $(<1 \%)$. In Chapter 4 of this thesis we describe the identification of a member of a novel $\beta$-lactamase family (termed blapsv-1) from Pseudovibrio ascidiaceicola strain 1D03 that was isolated from $A$. aerophoba. This enzyme conferred resistance to ampicillin and penicillin, and the most closely related known $\beta$-lactamases belonged to the families LEN and SHV, sharing $\sim 50 \%$ amino acid identity with blapsV-1. The closest nonPseudovibrio hit in NCBI's non-redundant protein sequences database is a $\beta$ lactamase from the marine bacterium Ruegeria atlantica with $63 \%$ amino acid identity. The $\beta$-lactamase from Ruegeria was computationally predicted and its 
functionality has not been experimentally verified. As such, the gene has not been assigned to a $\beta$-lactamase family. These results show that members of the Pseudovibrio genus, which appear to preferentially reside in sponges, contain resistance genes that so far have not been identified elsewhere. This may indicate that the enzyme converts a substrate that is exclusive to sponges (or sessile marine animals), but more importantly, it calls attention to sponges as a reservoir of novel resistance genes that can potentially be harnessed by non-sponge associated microorganisms including human pathogens. In Chapter 5 we detected proteins that were highly similar (>95\% amino acid identity) to blapsv-1 in 21 distinct $P$. ascidiaceicola strains that were isolated from the coast of Portugal, the coast of Spain, and in the Limski channel of Croatia. We speculate that the gene was acquired by an ancestral Pseudovibrio sp. strain in the Mediterranean region and that the gene since then has been conserved.

\section{Exchange of antibiotic resistance between aquatic niches and other, non-aquatic environments}

In the following we discuss important routes through which antibiotic resistance exchanges between the aquatic environment and the greater microbial biosphere. $\mathrm{A}$ distinction is made between the marine environment i.e. those habitats that depend on salt water from the sea, and the aquatic environment, which also includes freshwater habitats.

Remarkably, bla $a_{T E M-1}$, encoding the canonical TEM $\beta$-lactamase, which is notoriously found in human pathogens [446-448] and soil bacteria [449], was found to be prevalent in the marine environment. The gene was identified previously in the ocean [102], and we found this gene being expressed in the sponge Crambe crambe and in sea bacterioplankton (Chapter 2 of this thesis). Functional TEM $\beta$-lactamases have also been detected in river samples [103]. Bla TEM-1 was originally detected in $E$. coli isolated from a blood culture of a patient named Temoniera [450] and is often found on plasmids [448, 451, 452]. TEM-1 $\beta$-lactamases are widespread in the microbial biosphere, and as such their presence in the aquatic environment could have resulted from them spreading into the aquatic environment as a result of

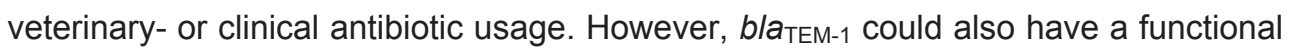
role in the aquatic environment as discussed in Chapter 2 of this thesis. 
In the aquatic environment, resistance genes have been detected in bacterial communities associated with microbialites [453], aquatic animals [454, 455], rivers [103, 456], the ocean [102], an estuary [457] and marine sediment [190]. All of these niches are in contact with sponges and can therefore serve as vehicles for resistance exchange between sponges and other biological niches. Resistance genes in aquatic bacteria have been predicted either to serve as defense against locally or selfproduced antimicrobials, or to have functional roles unrelated to resistance [295]. Alternatively, it cannot be excluded that the genes do not have a function in the natural environment, in which case resistance gene presence can be the result of prior anthropogenic antibiotic pressure. Resistance in the aquatic environment due to human activity can evolve as a result of antibiotic run-off or by dissemination of resistant bacteria from other niches [102]. In the latter case the resistance genes are expected to be present in non-marine taxa at least initially, but may spread to marine taxa via HGT. For example, it has been shown that downstream of a waste water treatment plant, the relative abundance of resistance determinants was higher as compared to upstream [103, 458]. In general, waste water treatment plants are considered hubs for HGT of resistance genes that can spread into the environment via effluent wastewater [99, 445, 459]. Furthermore, gulls have been proposed as an important reservoir for resistance gene dissemination due to their close association with humans and the fact that they may acquire resistant microorganisms by visiting farms or lagoons [460, 461]. Hasan and co-authors showed that $E$. coli isolates were resistant to more antibiotics if they were isolated from gulls that lived in an area with more human activity (e.g. more clinics, poultry farms and shrimp hatcheries) [462]. Gulls are often found at beaches and therefore can also carry resistance to the marine environment. Another study found elevated levels of antibiotic resistant bacteria in surface- and groundwater downstream of a swine facility [463]. Manure has been found to enrich antibiotic resistance in soil [101, 419, 464], and by the same token groundwater might become enriched for resistance [33, 445]. In addition, it has been shown that administration of tetracyclines to cattle increased the abundance of tetracycline-resistance-genes in associated waste water lagoons [465]. Yet another study indicated that faecal contamination of beaches might contribute to resistance in the marine environment [466]. Application of antibiotics in the aquatic environment is limited to the use of prophylactic antibiotics in aquaculture. Industrial aquaculture is rapidly growing [467], which is expected to concurrently increase 
antibiotic resistance frequencies in the marine environment [468-470]. For example, in sediment of the Northern Yellow Sea, the concentration of sulfonamides, which are highly persistent antibiotics that are used in local aquaculture, was found to be correlated with the relative abundance of resistance genes [471].

Taken together, these results show that the aquatic environment is a resistance reservoir of increasing importance in which the indigenous resistance gene diversity is being supplemented by resistance due to human activity. This may affect all biological niches in the marine environment, including sponges. Humans are directly exposed to these reservoirs via in situ bathing and via consumption of marine flora and fauna. Whether these routes of resistance dissemination pose an important threat to human health will require further research [472, 473].

\section{The human gut microbiota as antibiotic resistance reservoir}

An important reservoir from which pathogenic bacteria can acquire antibiotic resistance are human-associated bacteria, and particularly those residing in the gut $[80,474]$. Close contact between pathogens and the gut microbiota, especially in the densely populated large intestine, facilitates genetic exchange [426]. Mobilizable antibiotic resistance to clinical antibiotics in gut bacteria is mainly attributed to prior antibiotic usage [9], where resistance to new antibiotics usually develops within a few years after introduction $[475,476]$. In addition, gut bacteria harbour antibiotic resistance that is unrelated to the application of anthropogenic antibiotics [179-181]. Therefore, it is paramount to decipher the role of the human gut microbiota in the emergence of antibiotic resistance in pathogens. To comprehensively catalogue and characterize antibiotic resistance in human-associated bacteria, it is necessary to grow the bacteria up to pure culture. Metagenomic sequencing alone can never completely replace cultivation because it cannot be used to study all aspects of resistance and HGT mechanisms. However, a problem when attempting to obtain gut bacteria in pure culture is that a large fraction of them remains recalcitrant to cultivation [92, 477]. Another obstacle is that the gut of each individual human is expected to comprise $\sim 100-1,000$ different species [415-417], and that there are large inter-individual differences in terms of bacterial presence [416]. Therefore, exploring all microbial diversity will require considerable effort. Furthermore, it should also be taken into account that even if a strain of a certain species does not harbour resistance genes, it might be that another strain belonging to a subpopulation of the 
species actually does harbour resistance [478]. The high frequency at which HGT events occur in the gut microbiota should also be considered when studying resistance spread $[47,60]$. While conjugation is the main HGT mechanism through which resistance is transferred, transduction and transformation have also been implicated in resistance spread, and should not be neglected [47, 479]. Even though it is generally acknowledged that gut bacteria are (currently) largely uncultivable in vitro, recent studies provided evidence to the contrary. A study by Browne and colleagues, in which $\sim 4,000$ colonies from faecal samples of six healthy humans were re-streaked to purity, the cultured bacterial diversity comprised $96 \%$ and $90 \%$ of the bacterial abundance at the genus level and species level, respectively, based on average relative abundance across the six subjects [86]. Another study based on faecal material from two individuals retrieved $95 \%$ of order-level and $88 \%$ of familylevel taxonomic groups when growth across 10 different media was combined [390]. However, in case of the latter study it should be considered that bacteria may have relied on biotic factors from nearby bacteria to grow, as cultivation success was assessed based on growth on original plates containing large numbers of colonies [87]. In Chapter 6, we found that the faecal microbiota of 6 out of 18 human individuals correlated well with their respective growth communities on GAM and CP media with Pearson correlation values of $>0.6$. Growth communities derived from faeces from one individual even scored Pearson correlation values of $>0.95$. The less representative bacterial growth derived from faeces of the 12 remaining individuals may have resulted from a low viability of the samples due to cryo-preservation of faecal material in glycerol stocks before inoculation. Indeed, in the previouslymentioned studies that showed more representative bacterial growth [86, 390], fresh faecal material was inoculated within one hour after sample collection. In our study it was unpractical to use fresh faecal material because we were interested in analysing faecal material of a large number of intensive care patients. One hurdle in the isolation of novel taxa is their typically low relative abundance. Isolation of novel bacteria from the more abundant taxa will result in isolation of bacteria from novel taxa becoming progressively harder, thereby increasing the need for targeted approaches. A targeted approach can be implemented if cultivation conditions can be custom-designed based on genomic data. Genomic data can be obtained by cultureindependent genome sequencing of specific sub-populations or even single cells (e.g. after cell sorting [480]), or by extracting genomic data from metagenomic data 
by taking advantage of differences in genome coverage between bacteria (i.e. differential coverage binning) [94, 481]. If researchers simply aim to isolate members of novel taxa or novel bacteria with a specific phenotype, then the first step can be to analyse bacterial growth at the community level on different media that include selective conditions such as supplementation of the medium with antibiotics [390] or treatment of samples with ethanol [86]. If the screen yields novel taxa of interest, such initial screening can be followed-up by re-plating and subsequently picking a large number of colonies e.g. with the help of automated colony pickers [482]. This is also the approach that was used in this thesis to isolate an antibiotic resistant member of a taxon of which no members have yet been genome sequenced. The closest member of this bacterium is the recently described Sellimonas intestinalis $\mathrm{BR}^{\top} 2^{\top}$ [402], and its moderate prevalence in human gut microbiota of ca. $20 \%$ merits further investigation into this strain as potential antibiotic resistance reservoir (Figure 7.2). Recently, a group of bacteria comprising $>15 \%$ of groundwater bacteria was described and called the candidate phyla radiation [483], which taken together constitute a collection of $>35$ phyla that lack isolated representatives, and many of which would escape detection by $16 \mathrm{~S}$ rRNA targeted approaches due to mismatches

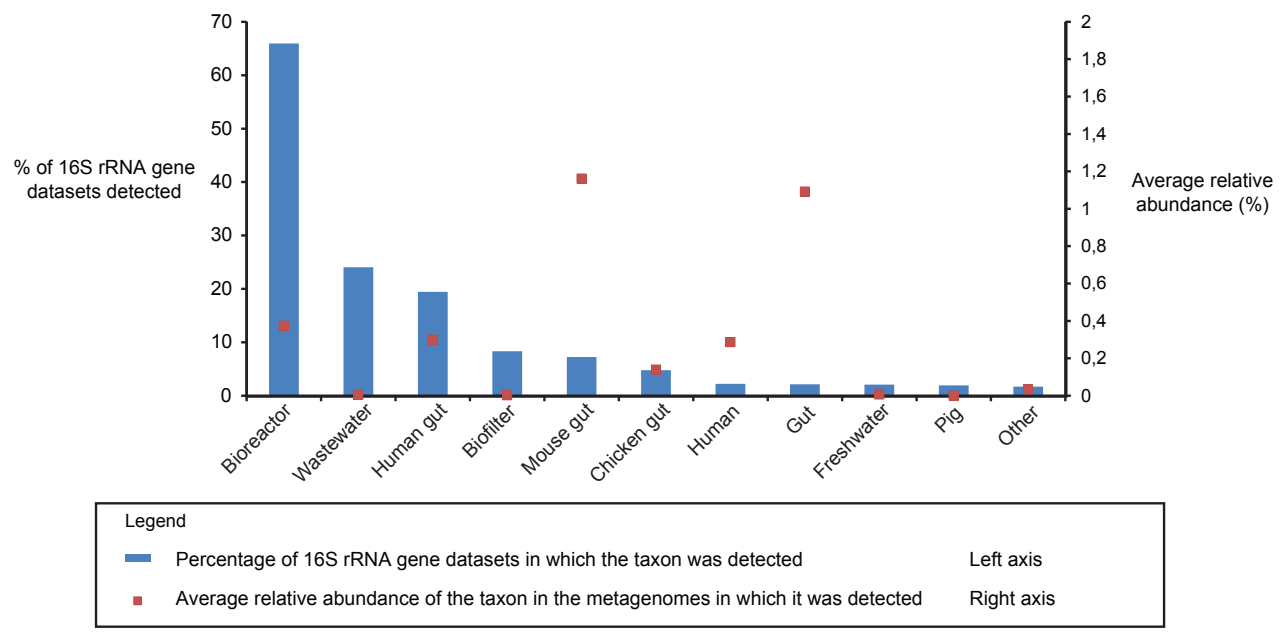

Figure 7.2 Using a targeted approach, a bacterium (OTU3088) was isolated on the MicroDish platform that shared 99\% $16 \mathrm{~S}$ rRNA gene identity with Sellimonas intestinalis BR72 ${ }^{\top}$. Relative abundance of this strain was derived from available 16S rRNA gene amplicon datasets in the SRA database. The analysis was performed by the IMNGS platform (https://www.imngs.org/about/), which divides the datasets in 105 categories according to which biological niche was sampled. For detection, a nearly full-length (1,399 bp) 16S rRNA gene sequence of OTU3088 was used as query. The similarity threshold was set at $99 \%$ nucleotide identity. All metagenome categories are shown in which the bacterium was detected at $>1,5 \%$ relative abundance. 
with commonly used "universal" primers and probes. They were identified by filtering groundwater samples through $\leq 0.2 \mu \mathrm{m}$ filters, followed by metagenomic sequencing of the filtrate. It was shown that they have limited metabolic capacity e.g. they can have incomplete tricarboxylic acid cycles, incomplete nucleotide biosynthesis pathways, or incomplete amino acid biosynthesis pathways. It is predicted that also human gut microbiota contain rare bacteria that could be hard to cultivate or are not detected by conventional $16 \mathrm{~S}$ rRNA profiling [484]. These rare bacteria may also be relevant with regards to the emergence of antibiotic resistance.

\section{Future outlook and main conclusion}

It has been predicted that the global annual death toll of antibiotic resistance will grow from 700,000 nowadays to 10 million in 2050 , superseding the death toll of road traffic accidents (1.2 million) and even cancer (8.2 million) [411]. The gravity of the situation demands that all viable strategies to combat emerging antibiotic resistance need to be treated with equal consideration, including development of novel antibiotics [485, 486], antibiotic stewardship programs [487, 488], restriction of agricultural antibiotic use [489, 490] and antibiotic cycling [491]. In this thesis we studied how the environment, and in particular sponge microbiota and anaerobic human gut microbiota, may contribute to the antibiotic crisis as a resistance reservoir. We applied complementary cultivation dependent and molecular methods to investigate antibiotic resistance at the community-level i.e. metatranscriptomics and functional metagenomics, and methods to investigate resistance at the level of individual species i.e. isolation of resistant bacteria and comparative genomics (Figure 7.1). The identification of a sponge-specific $\beta$-lactamase (blapsv-1) indicated that sponges may uniquely contribute to resistance in the environment. Furthermore, we conclude that the human gut microbiota potentially harbours numerous antibiotic resistance reservoir species that can be accessed by conventional cultivation, although their isolation should be preferably attempted with selective or targeted cultivation campaigns. To what extent the marine environment contributes to resistance in the clinics remains unknown; therefore, before considering intervention, marine resistance genes will need to be further catalogued, and their exchange mechanisms and factors that contribute to environmental spread will need to be studied in-depth. 



\section{References}

1. Klaus, K.; Steinwedel, C., Maggot Debridement Therapy: Advancing to the Past in Wound Care. Medsurg Nurs. 2015, 24, (6), 407-11.

2. Sun, X.; Jiang, K.; Chen, J.; Wu, L.; Lu, H.; Wang, A.; Wang, J., A systematic review of maggot debridement therapy for chronically infected wounds and ulcers. Int. J. Infect. Dis. 2014, 25, 32-7.

3. Ashkenazi, S., Beginning and possibly the end of the antibiotic era. J. Paediatr. Child Health 2013, 49, (3), E179-82.

4. Davies, J.; Davies, D., Origins and evolution of antibiotic resistance. Microbiol Mol Biol Rev 2010, 74, (3), 417-33.

5. Armstrong, G. L.; Conn, L. A.; Pinner, R. W., Trends in infectious disease mortality in the United States during the 20th century. JAMA 1999, 281, (1), 61-6.

6. Singh, S. B., Confronting the challenges of discovery of novel antibacterial agents. Bioorg Med Chem Lett 2014, 24, (16), 3683-9.

7. Bülow, E.; Gonzalez, T. B.; Versluis, D.; Oostdijk, E. A.; Ogilvie, L. A.; van Mourik, M. S.; Oosterink, E.; van Passel, M. W.; Smidt, H.; D'Andrea, M. M.; de Been, M.; Jones, B. V.; Willems, R. J.; Bonten, M. J.; van Schaik, W., Effects of selective digestive decontamination (SDD) on the gut resistome. $J$ Antimicrob Chemother 2014, 69, 2215-2223.

8. Davies, J., Vicious circles: looking back on resistance plasmids. Genetics 1995, 139, (4), 1465-8.

9. Jernberg, C.; Lofmark, S.; Edlund, C.; Jansson, J. K., Long-term impacts of antibiotic exposure on the human intestinal microbiota. Microbiology 2010, 156, (Pt 11), 3216-23.

10. Cooper, M. A.; Shlaes, D., Fix the antibiotics pipeline. Nature 2011, 472, (7341), 32.

11. Dautzenberg, M. J.; Ossewaarde, J. M.; de Kraker, M. E.; van der Zee, A.; van Burgh, S.; de Greeff, S. C.; Bijlmer, H. A.; Grundmann, H.; Cohen Stuart, J. W.; Fluit, A. C.; Troelstra, A.; Bonten, M. J., Successful control of a hospital-wide outbreak of OXA-48 producing Enterobacteriaceae in the Netherlands, 2009 to 2011. Euro Surveill. 2014, 19, (9).

12. Cohen, M. L., Epidemiology of drug resistance: implications for a post-antimicrobial era. Science 1992, 257, (5073), 1050-5.

13. Schwaber, M. J.; Carmeli, Y., Carbapenem-resistant Enterobacteriaceae: a potential threat. JAMA 2008, 300, (24), 2911-3.

14. Solomon, S. L.; Oliver, K. B., Antibiotic resistance threats in the United States: stepping back from the brink. Am. Fam. Physician 2014, 89, (12), 938-41.

15. Nieuwsbericht, Schippers: 16 miljoen extra voor onderzoek antibioticaresistentie. https://www.rijksoverheid.nl/ 2016.

16. Michael, C. A.; Dominey-Howes, D.; Labbate, M., The antimicrobial resistance crisis: causes, consequences, and management. Frontiers in public health 2014, 2, 145.

17. Gross, M., Antibiotics in crisis. Curr Biol 2013, 23, (24), R1063-5.

18. Centers for Disease Control and Prevention, Office of Infectious Disease Antibiotic resistance threats in the United States. http://www.cdc.gov/drugresistance/threat-report-2013 2013.

19. Sengupta, S.; Chattopadhyay, M. K.; Grossart, H. P., The multifaceted roles of antibiotics and antibiotic resistance in nature. Front. Microbiol. 2013, 4, 47.

20. Miller, W. R.; Murray, B. E.; Rice, L. B.; Arias, C. A., Vancomycin-Resistant enterococci: Therapeutic Challenges in the 21st Century. Infect. Dis. Clin. North Am. 2016, 30, (2), 415-39.

21. Adler, A.; Katz, D. E.; Marchaim, D., The Continuing Plague of Extended-spectrum beta-lactamaseproducing Enterobacteriaceae Infections. Infect. Dis. Clin. North Am. 2016, 30, (2), 347-75.

22. Kurz, S. G.; Furin, J. J.; Bark, C. M., Drug-Resistant Tuberculosis: Challenges and Progress. Infect. Dis. Clin. North Am. 2016, 30, (2), 509-22.

23. Rossolini, G. M.; Arena, F.; Pecile, P.; Pollini, S., Update on the antibiotic resistance crisis. Curr Opin Pharmacol 2014, 18, 56-60.

24. von Wintersdorff, C. J.; Penders, J.; Stobberingh, E. E.; Oude Lashof, A. M.; Hoebe, C. J.; Savelkoul, P. H.; Wolffs, P. F., High rates of antimicrobial drug resistance gene acquisition after international travel, The Netherlands. Emerg Infect Dis 2014, 20, (4), 649-57.

25. Bengtsson-Palme, J.; Angelin, M.; Huss, M.; Kjellqvist, S.; Kristiansson, E.; Palmgren, H.; Larsson, D. G. J.; Johanssone, A., The Human Gut Microbiome as a Transporter of Antibiotic Resistance Genes between Continents. Antimicrob Agents Chemother 2015, 59, (10), 6551-6560.

26. Jacquier, H.; Le Monnier, A.; Carbonnelle, E.; Corvec, S.; Illiaquer, M.; Bille, E.; Zahar, J. R.; Jaureguy, F.; Fihman, V.; Tankovic, J.; Cattoir, V.; Gmc Study, G., In vitro antimicrobial activity of "last-resort" antibiotics against unusual nonfermenting Gram-negative bacilli clinical isolates. Microb Drug Resist 2012, 18, (4), 396-401.

27. Liu, Y. Y.; Wang, Y.; Walsh, T. R.; Yi, L. X.; Zhang, R.; Spencer, J.; Doi, Y.; Tian, G.; Dong, B.; Huang, X.; Yu, L. F.; Gu, D.; Ren, H.; Chen, X.; Lv, L.; He, D.; Zhou, H.; Liang, Z.; Liu, J. H.; Shen, J., Emergence of plasmid-mediated colistin resistance mechanism MCR-1 in animals and human beings in China: a microbiological and molecular biological study. Lancet Infect. Dis. 2016, 16, (2), 161-8.

28. Ye, H.; Li, Y.; Li, Z.; Gao, R.; Zhang, H.; Wen, R.; Gao, G. F.; Hu, Q.; Feng, Y., Diversified mcr-1Harbouring Plasmid Reservoirs Confer Resistance to Colistin in Human Gut Microbiota. mBio 2016, 7, (2).

29. Hasman, H.; Hammerum, A. M.; Hansen, F.; Hendriksen, R. S.; Olesen, B.; Agerso, Y.; Zankari, E.; Leekitcharoenphon, P.; Stegger, M.; Kaas, R. S.; Cavaco, L. M.; Hansen, D. S.; Aarestrup, F. M.; Skov, R. L., Detection of mcr-1 encoding plasmid-mediated colistin-resistant Escherichia coli isolates from human bloodstream infection and imported chicken meat, Denmark 2015. Euro Surveill. 2015, 20, (49). 
30. Olaitan, A. O.; Chabou, S.; Okdah, L.; Morand, S.; Rolain, J. M., Dissemination of the mcr-1 colistin resistance gene. Lancet Infect. Dis. 2016, 16, (2), 147.

31. Fishman, N., Antimicrobial stewardship. Am J Infect Control 2006, 34, (5 Suppl 1), S55-63; discussion S64-73.

32. Luyt, C. E.; Brechot, N.; Trouillet, J. L.; Chastre, J., Antibiotic stewardship in the intensive care unit. Crit. Care 2014, 18, (5), 480.

33. Kemper, N., Veterinary antibiotics in the aquatic and terrestrial environment. Ecol. Indicators 2008, 8, $(1), 1-13$

34. Mevius, D.; Hellebrekers, L. J., [Antibiotics in animal husbandry: a thorny problem]. Ned. Tijdschr. Geneeskd. 2010, 154, (45), B629.

35. Ling, L. L.; Schneider, T.; Peoples, A. J.; Spoering, A. L.; Engels, I.; Conlon, B. P.; Mueller, A.; Schaberle, T. F.; Hughes, D. E.; Epstein, S.; Jones, M.; Lazarides, L.; Steadman, V. A.; Cohen, D. R.; Felix, C. R.; Fetterman, K. A.; Millett, W. P.; Nitti, A. G.; Zullo, A. M.; Chen, C.; Lewis, K., A new antibiotic kills pathogens without detectable resistance. Nature 2015, 517, (7535), 455-+.

36. Renwick, M. J.; Brogan, D. M.; Mossialos, E., A systematic review and critical assessment of incentive strategies for discovery and development of novel antibiotics. J Antibiot (Tokyo) 2016, 69, (2), 73-88.

37. Berendonk, T. U.; Manaia, C. M.; Merlin, C.; Fatta-Kassinos, D.; Cytryn, E.; Walsh, F.; Burgmann, H.; Sorum, H.; Norstrom, M.; Pons, M. N.; Kreuzinger, N.; Huovinen, P.; Stefani, S.; Schwartz, T.; Kisand, V.; Baquero, F.; Martinez, J. L., Tackling antibiotic resistance: the environmental framework. Nat. Rev. Microbiol. 2015, 13, (5), 310-7.

38. De Gelder, L.; Williams, J. J.; Ponciano, J. M.; Sota, M.; Top, E. M., Adaptive plasmid evolution results in host-range expansion of a broad-host-range plasmid. Genetics 2008, 178, (4), 2179-90.

39. Walsh, T. R.; Weeks, J.; Livermore, D. M.; Toleman, M. A., Dissemination of NDM-1 positive bacteria in the New Delhi environment and its implications for human health: an environmental point prevalence study. Lancet Infect. Dis. 2011, 11, (5), 355-62.

40. Martinez, J. L., Antibiotics and antibiotic resistance genes in natural environments. Science 2008, 321, (5887), 365-7.

41. Hasman, H.; Aarestrup, F. M.; Dalsgaard, A.; Guardabassi, L., Heterologous expression of glycopeptide resistance vanHAX gene clusters from soil bacteria in Enterococcus faecalis. $J$ Antimicrob Chemother 2006, 57, (4), 648-53.

42. Torres-Cortes, G.; Millan, V.; Ramirez-Saad, H. C.; Nisa-Martinez, R.; Toro, N.; Martinez-Abarca, F., Characterization of novel antibiotic resistance genes identified by functional metagenomics on soil samples. Environ. Microbiol. 2011, 13, (4), 1101-14.

43. Forsberg, K. J.; Reyes, A.; Wang, B.; Selleck, E. M.; Sommer, M. O.; Dantas, G., The shared antibiotic resistome of soil bacteria and human pathogens. Science 2012, 337, (6098), 1107-11.

44. Kruse, T.; Levisson, M.; de Vos, W. M.; Smidt, H., vanI: a novel D-Ala-D-Lac vancomycin resistance gene cluster found in Desulfitobacterium hafniense. Microb Biotechnol 2014, 7, (5), 456-466.

45. Allen, H. K.; Donato, J.; Wang, H. H.; Cloud-Hansen, K. A.; Davies, J.; Handelsman, J., Call of the wild: antibiotic resistance genes in natural environments. Nature Rev Microbiol 2010, 8, (4), 251-259.

46. Aminov, R. I.; Mackie, R. I., Evolution and ecology of antibiotic resistance genes. FEMS Microbiol Lett 2007, 271, (2), 147-61.

47. Huddleston, J. R., Horizontal gene transfer in the human gastrointestinal tract: potential spread of antibiotic resistance genes. Infection and drug resistance 2014, 7, 167-76.

48. von Wintersdorff, C. J. H.; Penders, J.; van Niekerk, J. M.; Mills, N. D.; Majumder, S.; van Alphen, L. B.; Savelkoul, P. H. M.; Wolffs, P. F. G., Dissemination of Antimicrobial Resistance in Microbial Ecosystems through Horizontal Gene Transfer. Front. Microbiol. 2016, 7.

49. Soucy, S. M.; Huang, J.; Gogarten, J. P., Horizontal gene transfer: building the web of life. Nat Rev Genet 2015, 16, (8), 472-82.

50. Ochman, H.; Lawrence, J. G.; Groisman, E. A., Lateral gene transfer and the nature of bacterial innovation. Nature 2000, 405, (6784), 299-304.

51. Carattoli, A., Plasmids and the spread of resistance. Int. J. Med. Microbiol. 2013, 303, (6-7), $298-304$.

52. Thomas, C. M.; Nielsen, K. M., Mechanisms of, and barriers to, horizontal gene transfer between bacteria. Nature Reviews Microbiology 2005, 3, (9), 711-721.

53. Barlow, M., What antimicrobial resistance has taught us about horizontal gene transfer. Methods Mol Biol 2009, 532, 397-411.

54. Stoesser, N.; Sheppard, A. E.; Peirano, G.; Sebra, R.; Lynch, T.; Anson, L.; Kasarskis, A.; Motyl, M. R.; Crook, D. W.; Pitout, J. D., First report of blaIMP-14 on a plasmid harboring multiple drug resistance genes in Escherichia coli ST131. Antimicrob Agents Chemother 2016.

55. Nakamura, Y.; Itoh, T.; Matsuda, H.; Gojobori, T., Biased biological functions of horizontally transferred genes in prokaryotic genomes. Nat Genet 2004, 36, (7), 760-6.

56. McDaniel, L. D.; Young, E.; Delaney, J.; Ruhnau, F.; Ritchie, K. B.; Paul, J. H., High frequency of horizontal gene transfer in the oceans. Science 2010, 330, (6000), 50.

57. Wiedenbeck, J.; Cohan, F. M., Origins of bacterial diversity through horizontal genetic transfer and adaptation to new ecological niches. FEMS Microbiol Rev 2011, 35, (5), 957-976.

58. Rest, J. S.; Mindell, D. P., Retroids in archaea: phylogeny and lateral origins. Mol Biol Evol 2003, 20, (7), 1134-42.

59. Garcia-Vallve, S.; Romeu, A.; Palau, J., Horizontal gene transfer of glycosyl hydrolases of the rumen fungi. Mol Biol Evol 2000, 17, (3), 352-61.

60. Summers, A. O., Genetic linkage and horizontal gene transfer, the roots of the antibiotic multiresistance problem. Anim Biotechnol 2006, 17, (2), 125-35.

61. Kohanski, M. A.; Dwyer, D. J.; Collins, J. J., How antibiotics kill bacteria: from targets to networks. Nature Reviews Microbiology 2010, 8, (6), 423-435. 
62. Van Boeckel, T. P.; Gandra, S.; Ashok, A.; Caudron, Q.; Grenfell, B. T.; Levin, S. A.; Laxminarayan, R., Global antibiotic consumption 2000 to 2010: an analysis of national pharmaceutical sales data. Lancet Infect. Dis. 2014, 14, (8), 742-50.

63. Poole, K., Resistance to beta-lactam antibiotics. Cell. Mol. Life Sci. 2004, 61, (17), 2200-23.

64. Higgins, P. G.; Fluit, A. C.; Schmitz, F. J., Fluoroquinolones: structure and target sites. Curr Drug Targets 2003, 4, (2), 181-90.

65. Coque, T. M.; Singh, K. V.; Weinstock, G. M.; Murray, B. E., Characterization of dihydrofolate reductase genes from trimethoprim-susceptible and trimethoprim-resistant strains of Enterococcus faecalis. Antimicrob Agents Chemother 1999, 43, (1), 141-147.

66. Falagas, M. E.; Kasiakou, S. K., Colistin: the revival of polymyxins for the management of multidrugresistant Gram-negative bacterial infections. Clin Infect Dis 2005, 40, (9), 1333-41.

67. Mirrett, S.; Reller, L. B.; Knapp, J. S., Neisseria gonorrhoeae strains inhibited by vancomycin in selective media and correlation with auxotype. J. Clin. Microbiol. 1981, 14, (1), 94-9.

68. Livermore, D. M., Linezolid in vitro: mechanism and antibacterial spectrum. J Antimicrob Chemother 2003, 51 Suppl 2, ii9-16.

69. Schmieder, R.; Edwards, R., Insights into antibiotic resistance through metagenomic approaches. Future Microbiol. 2012, 7, (1), 73-89.

70. McManus, M. C., Mechanisms of bacterial resistance to antimicrobial agents. Am. J. Health Syst. Pharm. 1997, 54, (12), 1420-33; quiz 1444-6.

71. Tetz, G.; Tetz, V.; Vecherkovskaya, M., Genomic characterization and assessment of the virulence and antibiotic resistance of the novel species Paenibacillus sp. strain VT-400, a potentially pathogenic bacterium in the oral cavity of patients with hematological malignancies. Gut Pathog. 2016, 8.

72. Schenk, M. F.; Witte, S.; Salverda, M. L. M.; Koopmanschap, B.; Krug, J.; de Visser, J. A. G. M., Role of pleiotropy during adaptation of TEM-1 beta-lactamase to two novel antibiotics. Evol Appl 2015, 8, (3), 248-260.

73. Wiesner, M.; Fernandez-Mora, M.; Cevallos, M. A.; Zavala-Alvarado, C.; Zaidi, M. B.; Calva, E.; Silva, C., Conjugative transfer of an IncA/C plasmid-borne bla(CMY-2) gene through genetic rearrangements with an IncX1 plasmid. BMC Microbiol 2013, 13.

74. Wright, G. D., The antibiotic resistome: the nexus of chemical and genetic diversity. Nature Reviews Microbiology 2007, 5, (3), 175-186.

75. Perreten, V.; Vorlet-Fawer, L.; Slickers, P.; Ehricht, R.; Kuhnert, P.; Frey, J., Microarray-based detection of 90 antibiotic resistance genes of Gram-positive bacteria. J. Clin. Microbiol. 2005, 43, (5), 2291-302.

76. Card, R.; Zhang, J. C.; Das, P.; Cook, C.; Woodford, N.; Anjum, M. F., Evaluation of an Expanded Microarray for Detecting Antibiotic Resistance Genes in a Broad Range of Gram-negative Bacterial Pathogens. Antimicrob Agents Chemother 2013, 57, (1), 458-465.

77. Bockelmann, U.; Dorries, H. H.; Ayuso-Gabella, M. N.; de Marcay, M. S.; Tandoi, V.; Levantesi, C.; Masciopinto, C.; Van Houtte, E.; Szewzyk, U.; Wintgens, T.; Grohmann, E., Quantitative PCR Monitoring of Antibiotic Resistance Genes and Bacterial Pathogens in Three European Artificial Groundwater Recharge Systems. Appl Environ Microbiol 2009, 75, (1), 154-163.

78. Volkmann, H.; Schwartz, T.; Bischoff, P.; Kirchen, S.; Obst, U., Detection of clinically relevant antibiotic-resistance genes in municipal wastewater using real-time PCR (TaqMan). J Microbiol Methods 2004, 56, (2), 277-286.

79. Pehrsson, E. C.; Forsberg, K. J.; Gibson, M. K.; Ahmadi, S.; Dantas, G., Novel resistance functions uncovered using functional metagenomic investigations of resistance reservoirs. Front. Microbiol. 2013, 4.

80. van Schaik, W., The human gut resistome. Philos Trans R Soc Lond B Biol Sci 2015, 370, (1670), 20140087.

81. Provenzano, J. C.; Siqueira, J. F.; Rocas, I. N.; Domingues, R. R.; Leme, A. F. P.; Silva, M. R. S., Metaproteome Analysis of Endodontic Infections in Association with Different Clinical Conditions. PLOS One 2013, 8, (10).

82. Maurice, C. F.; Haiser, H. J.; Turnbaugh, P. J., Xenobiotics Shape the Physiology and Gene Expression of the Active Human Gut Microbiome. Cell 2013, 152, (1-2), 39-50.

83. Staley, J. T.; Konopka, A., Measurement of in situ activities of nonphotosynthetic microorganisms in aquatic and terrestrial habitats. Annu Rev Microbiol 1985, 39, 321-46.

84. Hugenholtz, P.; Goebel, B. M.; Pace, N. R., Impact of culture-independent studies on the emerging phylogenetic view of bacterial diversity. J Bacteriol 1998, 180, (18), 4765-74.

85. Pfleiderer, A.; Lagier, J. C.; Armougom, F.; Robert, C.; Vialettes, B.; Raoult, D., Culturomics identified 11 new bacterial species from a single anorexia nervosa stool sample. Eur. J. Clin. Microbiol. Infect. Dis. 2013.

86. Browne, H. P.; Forster, S. C.; Anonye, B. O.; Kumar, N.; Neville, B. A.; Stares, M. D.; Goulding, D.; Lawley, T. D., Culturing of 'unculturable' human microbiota reveals novel taxa and extensive sporulation. Nature 2016, 533, (7604), 543-6.

87. Pham, V. H.; Kim, J., Cultivation of unculturable soil bacteria. Trends Biotechnol 2012, 30, (9), 47584.

88. Crocetti, G. R.; Banfield, J. F.; Keller, J.; Bond, P. L.; Blackall, L. L., Glycogen-accumulating organisms in laboratory-scale and full-scale wastewater treatment processes. Microbiology 2002, 148, (Pt 11), 3353-64.

89. Ingham, C. J.; Sprenkels, A.; Bomer, J.; Molenaar, D.; van den Berg, A.; van Hylckama Vlieg, J. E.; de Vos, W. M., The micro-Petri dish, a million-well growth chip for the culture and high-throughput screening of microorganisms. Proc Natl Acad Sci U S A 2007, 104, (46), 18217-22. 
90. Ferrari, B. C.; Binnerup, S. J.; Gillings, M., Microcolony cultivation on a soil substrate membrane system selects for previously uncultured soil bacteria. Appl Environ Microbiol 2005, 71, (12), 8714-20.

91. Bollmann, A.; Lewis, K.; Epstein, S. S., Incubation of environmental samples in a diffusion chamber increases the diversity of recovered isolates. Appl Environ Microbio/ 2007, 73, (20), 6386-90.

92. Stewart, E. J., Growing unculturable bacteria. J Bacteriol 2012, 194, (16), 4151-60.

93. Alain, K.; Querellou, J., Cultivating the uncultured: limits, advances and future challenges. Extremophiles 2009, 13, (4), 583-94.

94. Albertsen, M.; Hugenholtz, P.; Skarshewski, A.; Nielsen, K. L.; Tyson, G. W.; Nielsen, P. H., Genome sequences of rare, uncultured bacteria obtained by differential coverage binning of multiple metagenomes. Nat Biotechnol 2013, 31, (6), 533-+.

95. Tripp, H. J.; Kitner, J. B.; Schwalbach, M. S.; Dacey, J. W.; Wilhelm, L. J.; Giovannoni, S. J., SAR11 marine bacteria require exogenous reduced sulphur for growth. Nature 2008, 452, (7188), 741-4.

96. Tyson, G. W.; Lo, I.; Baker, B. J.; Allen, E. E.; Hugenholtz, P.; Banfield, J. F., Genome-directed isolation of the key nitrogen fixer Leptospirillum ferrodiazotrophum sp. nov. from an acidophilic microbial community. Appl Environ Microbiol 2005, 71, (10), 6319-24.

97. Durso, L. M.; Miller, D. N.; Wienhold, B. J., Distribution and quantification of antibiotic resistant genes and bacteria across agricultural and non-agricultural metagenomes. PLoS One 2012, 7, (11), e48325.

98. Mori, T.; Mizuta, S.; Suenaga, H.; Miyazaki, K., Metagenomic screening for bleomycin resistance genes. Appl Environ Microbiol 2008, 74, (21), 6803-5.

99. Munck, C.; Albertsen, M.; Telke, A.; Ellabaan, M.; Nielsen, P. H.; Sommer, M. O., Limited dissemination of the wastewater treatment plant core resistome. Nature communications 2015, 6, 8452.

100. Alves, M. S.; Pereira, A.; Araujo, S. M.; Castro, B. B.; Correia, A. C.; Henriques, I., Seawater is a reservoir of multi-resistant Escherichia coli, including strains hosting plasmid-mediated quinolones resistance and extended-spectrum beta-lactamases genes. Front. Microbiol. 2014, 5, 426.

101. Wichmann, F.; Udikovic-Kolic, N.; Andrew, S.; Handelsman, J., Diverse antibiotic resistance genes in dairy cow manure. mBio 2014, 5, (2), e01017.

102. Hatosy, S. M.; Martiny, A. C., The Ocean as a Global Reservoir of Antibiotic Resistance Genes. Appl Environ Microbiol 2015, 81, (21), 7593-9.

103. Amos, G. C.; Zhang, L.; Hawkey, P. M.; Gaze, W. H.; Wellington, E. M., Functional metagenomic analysis reveals rivers are a reservoir for diverse antibiotic resistance genes. Vet Microbiol 2014, 171, (3-4), 441-7.

104. Forslund, K.; Sunagawa, S.; Roat Kultima, J.; Mende, D.; Arumugam, M.; Typas, A.; Bork, P., Country-specific antibiotic use practices impact the human gut resistome. Genome Res 2013, 23, 1163-1169.

105. Gibson, M. K.; Forsberg, K. J.; Dantas, G., Improved annotation of antibiotic resistance determinants reveals microbial resistomes cluster by ecology. Isme J 2014, 9, 207-216.

106. Pehrsson, E. C.; Tsukayama, P.; Patel, S.; Mejia-Bautista, M.; Sosa-Soto, G.; Navarrete, K. M.; Calderon, M.; Cabrera, L.; Hoyos-Arango, W.; Bertoli, M. T.; Berg, D. E.; Gilman, R. H.; Dantas, G., Interconnected microbiomes and resistomes in low-income human habitats. Nature 2016, 533, (7602), 212-6.

107. Noyes, N. R.; Yang, X.; Linke, L. M.; Magnuson, R. J.; Dettenwanger, A.; Cook, S.; Geornaras, I.; Woerner, D. E.; Gow, S. P.; McAllister, T. A.; Yang, H.; Ruiz, J.; Jones, K. L.; Boucher, C. A.; Morley, P. S.; Belk, K. E., Resistome diversity in cattle and the environment decreases during beef production. eLife 2016, 5.

108. Bhullar, K.; Waglechner, N.; Pawlowski, A.; Koteva, K.; Banks, E. D.; Johnston, M. D.; Barton, H. A.; Wright, G. D., Antibiotic resistance is prevalent in an isolated cave microbiome. PLoS One 2012, 7 , (4), e34953.

109. D'Costa, V. M.; King, C. E.; Kalan, L.; Morar, M.; Sung, W. W. L.; Schwarz, C.; Froese, D.; Zazula, G.; Calmels, F.; Debruyne, R.; Golding, G. B.; Poinar, H. N.; Wright, G. D., Antibiotic resistance is ancient. Nature 2011, 477, (7365), 457-461.

110. Rossolini, G. M.; D'Andrea, M. M.; Mugnaioli, C., The spread of CTX-M-type extended-spectrum betalactamases. Clin. Microbiol. Infect. 2008, 14 Suppl 1, 33-41.

111. Bonnet, R., Growing group of extended-spectrum beta-lactamases: the CTX-M enzymes. Antimicrob Agents Chemother 2004, 48, (1), 1-14.

112. Jacoby, G. A.; Hooper, D. C., Phylogenetic analysis of chromosomally determined qnr and related proteins. Antimicrob Agents Chemother 2013, 57, (4), 1930-4.

113. Canton, R., Antibiotic resistance genes from the environment: a perspective through newly identified antibiotic resistance mechanisms in the clinical setting. Clin. Microbiol. Infect. 2009, 15, 20-25.

114. Whitman, W. B.; Coleman, D. C.; Wiebe, W. J., Prokaryotes: The unseen majority. Proc Natl Acad Sci U S A 1998, 95, (12), 6578-6583.

115. Fuhrman, J. A.; Sleeter, T. D.; Carlson, C. A.; Proctor, L. M., Dominance of Bacterial Biomass in the Sargasso Sea and Its Ecological Implications. Mar Ecol Prog Ser 1989, 57, (3), 207-217.

116. Li, C. W.; Chen, J. Y.; Hua, T. E., Precambrian sponges with cellular structures. Science 1998, 279, (5352), 879-82.

117. Li, Z. Y.; Wang, Y. Z.; He, L. M.; Zheng, H. J., Metabolic profiles of prokaryotic and eukaryotic communities in deep-sea sponge Lamellomorpha sp. indicated by metagenomics. Sci. Rep. 2014, 4, 3895.

118. Thomas, T.; Moitinho-Silva, L.; Lurgi, M.; Bjork, J. R.; Easson, C.; Astudillo-Garcia, C.; Olson, J. B.; Erwin, P. M.; Lopez-Legentil, S.; Luter, H.; Chaves-Fonnegra, A.; Costa, R.; Schupp, P. J.; Steindler, L.; Erpenbeck, D.; Gilbert, J.; Knight, R.; Ackermann, G.; Victor Lopez, J.; Taylor, M. W.; Thacker, R. 
W.; Montoya, J. M.; Hentschel, U.; Webster, N. S., Diversity, structure and convergent evolution of the global sponge microbiome. Nature communications 2016, 7, 11870.

119. Osinga, R.; Armstrong, E.; Burgess, J. G.; Hoffmann, F.; Reitner, J.; Schumann-Kindel, G., Spongemicrobe associations and their importance for sponge bioprocess engineering. Hydrobiologia 2001, $461,55-62$.

120. Shieh, W. Y.; Lin, Y. M., Association of Heterotrophic Nitrogen-Fixing Bacteria with a Marine Sponge of Halichondria sp. Bull Mar Sci 1994, 54, (2), 557-564.

121. Diaz, M. C.; Ward, B. B., Sponge-mediated nitrification in tropical benthic communities. Mar Ecol Prog Ser 1997, 156, 97-107.

122. Wilkinson, C. R., Net primary productivity in coral reef sponges. Science 1983, 219, (4583), 410-2.

123. Webster, N. S.; Taylor, M. W., Marine sponges and their microbial symbionts: love and other relationships. Environ. Microbiol. 2012, 14, (2), 335-346.

124. Hentschel, U.; Usher, K. M.; Taylor, M. W., Marine sponges as microbial fermenters. FEMS Microbiol Ecol 2006, 55, (2), 167-177.

125. Taylor, M. W.; Radax, R.; Steger, D.; Wagner, M., Sponge-associated microorganisms: evolution, ecology, and biotechnological potential. Microbiol Mol Biol Rev 2007, 71, (2), 295-347.

126. Schmitt, S.; Tsai, P.; Bell, J.; Fromont, J.; Ilan, M.; Lindquist, N.; Perez, T.; Rodrigo, A.; Schupp, P. J.; Vacelet, J.; Webster, N.; Hentschel, U.; Taylor, M. W., Assessing the complex sponge microbiota: core, variable and species-specific bacterial communities in marine sponges. Isme J 2012, 6, (3), 564-576.

127. Lee, O. O.; Wang, Y.; Yang, J.; Lafi, F. F.; Al-Suwailem, A.; Qian, P. Y., Pyrosequencing reveals highly diverse and species-specific microbial communities in sponges from the Red Sea. Isme J 2011, 5, (4), 650-64.

128. Easson, C. G.; Thacker, R. W., Phylogenetic signal in the community structure of host-specific microbiomes of tropical marine sponges. Front. Microbiol. 2014, 5, 532.

129. Sipkema, D.; Schippers, K.; Maalcke, W. J.; Yang, Y.; Salim, S.; Blanch, H. W., Multiple approaches to enhance the cultivability of bacteria associated with the marine sponge Haliclona (gellius) sp. Appl Environ Microbiol 2011, 77, (6), 2130-40.

130. Montalvo, N. F.; Davis, J.; Vicente, J.; Pittiglio, R.; Ravel, J.; Hill, R. T., Integration of Culture-Based and Molecular Analysis of a Complex Sponge-Associated Bacterial Community. PLoS One 2014, 9, (3).

131. Steinert, G.; Whitfield, S.; Taylor, M. W.; Thoms, C.; Schupp, P. J., Application of diffusion growth chambers for the cultivation of marine sponge-associated bacteria. Mar. Biotechnol. (N. Y.) 2014, 16, (5), 594-603.

132. Santos-Gandelman, J. F.; Giambiagi-deMarval, M.; Oelemann, W. M.; Laport, M. S., Biotechnological potential of sponge-associated bacteria. Curr Pharm Biotechnol 2014, 15, (2), 143-55.

133. Faulkner, D. J., Marine natural products. Nat Prod Rep 2000, 17, (1), 7-55.

134. Mehbub, M. F.; Lei, J.; Franco, C.; Zhang, W., Marine Sponge Derived Natural Products between 2001 and 2010: Trends and Opportunities for Discovery of Bioactives. Mar. Drugs 2014, 12, (8), 45394577.

135. Indraningrat, A. A.; Smidt, H.; Sipkema, D., Bioprospecting Sponge-Associated Microbes for Antimicrobial Compounds. Mar. Drugs 2016, 14, (5).

136. Leal, M. C.; Puga, J.; Serodio, J.; Gomes, N. C.; Calado, R., Trends in the discovery of new marine natural products from invertebrates over the last two decades--where and what are we bioprospecting? PLoS One 2012, 7, (1), e30580.

137. Thoms, C.; Schupp, P., Biotechnological Potential of Marine Sponges and their Associated Bacteria as Producers of New Pharmaceuticals (Part II) J. Int. Biotechnol. Law 2005, 2, (6), 257-264.

138. Paul, V. J.; Puglisi, M. P.; Ritson-Williams, R., Marine chemical ecology. Nat Prod Rep 2006, 23, (2), 153-80.

139. Phelan, R. W.; Clarke, C.; Morrissey, J. P.; Dobson, A. D. W.; O'Gara, F.; Barbosa, T. M., Tetracycline resistance-encoding plasmid from Bacillus sp. strain \#24, isolated from the marine sponge Haliclona simulans. Appl Environ Microbiol 2011, 77, (1), 327-329.

140. Barbosa, T. M.; Phelan, R. W.; Leong, D.; Morrissey, J. P.; Adams, C.; Dobson, A. D.; O'Gara, F., A novel erythromycin resistance plasmid from Bacillus sp. strain HS24, isolated from the marine sponge Haliclona simulans. PLoS One 2014, 9, (12), e115583.

141. Bebell, L. M.; Muiru, A. N., Antibiotic use and emerging resistance: how can resource-limited countries turn the tide? Global heart 2014, 9, (3), 347-58.

142. Laport, M. S.; Pontes, P. V.; Dos Santos, D. S.; Santos-Gandelman, J. F.; Muricy, G.; Bauwens, M.; Giambiagi-deMarval, M.; George, I., Antibiotic resistance genes detected in the marine sponge Petromica citrina from Brazilian coast. Braz J Microbiol 2016.

143. Shieh, W. Y.; Lin, Y. T.; Jean, W. D., Pseudovibrio denitrificans gen. nov., sp. nov., a marine, facultatively anaerobic, fermentative bacterium capable of denitrification. Int J Syst Evol Microbiol 2004, 54, (Pt 6), 2307-12.

144. Xu, Y.; Li, Q.; Tian, R.; Lai, Q.; Zhang, Y., Pseudovibrio hongkongensis sp. nov., isolated from a marine flatworm. Antonie Leeuwenhoek 2015, 108, (1), 127-32.

145. Zhang, Y.; Li, Q.; Tian, R.; Lai, Q.; Xu, Y., Pseudovibrio stylochi sp. nov., isolated from a marine flatworm. Int J Syst Evol Microbiol 2016.

146. Lafi, F. F.; Garson, M. J.; Fuerst, J. A., Culturable bacterial symbionts isolated from two distinct sponge species (Pseudoceratina clavata and Rhabdastrella globostellata) from the Great Barrier Reef display similar phylogenetic diversity. Microb Ecol 2005, 50, (2), 213-20.

147. Menezes, C. B.; Bonugli-Santos, R. C.; Miqueletto, P. B.; Passarini, M. R.; Silva, C. H.; Justo, M. R.; Leal, R. R.; Fantinatti-Garboggini, F.; Oliveira, V. M.; Berlinck, R. G.; Sette, L. D., Microbial diversity associated with algae, ascidians and sponges from the north coast of Sao Paulo state, Brazil. Microbiol Res 2010, 165, (6), 466-82. 
148. Muscholl-Silberhorn, A.; Thiel, V.; Imhoff, J. F., Abundance and bioactivity of cultured spongeassociated bacteria from the Mediterranean sea. Microb Ecol 2008, 55, (1), 94-106.

149. Webster, N. S.; Hill, R. T., The culturable microbial community of the Great Barrier Reef sponge Rhopaloeides odorabile is dominated by an alpha-Proteobacterium. Mar. Biol. 2001, 138, (4), 843851.

150. Enticknap, J. J.; Kelly, M.; Peraud, O.; Hill, R. T., Characterization of a culturable alphaproteobacterial symbiont common to many marine sponges and evidence for vertical transmission via sponge larvae. Appl Environ Microbiol 2006, 72, (5), 3724-32.

151. Bondarev, V.; Richter, M.; Romano, S.; Piel, J.; Schwedt, A.; Schulz-Vogt, H. N., The genus Pseudovibrio contains metabolically versatile bacteria adapted for symbiosis. Environ. Microbiol. 2013, 15, (7), 2095-113.

152. Fukunaga, Y.; Kurahashi, M.; Tanaka, K.; Yanagi, K.; Yokota, A.; Harayama, S., Pseudovibrio ascidiaceicola sp. nov., isolated from ascidians (sea squirts). Int J Syst Evol Microbiol 2006, 56, (Pt 2), 343-7.

153. Riesenfeld, C. S.; Murray, A. E.; Baker, B. J., Characterization of the microbial community and polyketide biosynthetic potential in the palmerolide-producing tunicate Synoicum adareanum. J. Nat. Prod. 2008, 71, (11), 1812-8.

154. Sertan-de Guzman, A. A.; Predicala, R. Z.; Bernardo, E. B.; Neilan, B. A.; Elardo, S. P.; Mangalindan, G. C.; Tasdemir, D.; Ireland, C. M.; Barraquio, W. L.; Concepcion, G. P., Pseudovibrio denitrificans strain Z143-1, a heptylprodigiosin-producing bacterium isolated from a Philippine tunicate. FEMS Microbiol Lett 2007, 277, (2), 188-96.

155. Chen, Y. H.; Kuo, J.; Sung, P. J.; Chang, Y. C.; Lu, M. C.; Wong, T. Y.; Liu, J. K.; Weng, C. F.; Twan, W. H.; Kuo, F. W., Isolation of marine bacteria with antimicrobial activities from cultured and fieldcollected soft corals. World J Microbiol Biotechnol 2012, 28, (12), 3269-79.

156. Vizcaino, M. I., The chemical defense of Pseudopterogorgia americana: a focus on the antimicrobial potential of a Pseudovibrio sp. PhD Thesis 2011.

157. Hosoya, S.; Yokota, A., Pseudovibrio japonicus sp. nov., isolated from coastal seawater in Japan. Int J Syst Evol Microbiol 2007, 57, (Pt 9), 1952-5.

158. Santos, O. C.; Pontes, P. V.; Santos, J. F.; Muricy, G.; Giambiagi-deMarval, M.; Laport, M. S., Isolation, characterization and phylogeny of sponge-associated bacteria with antimicrobial activities from Brazil. Res Microbiol 2010, 161, (7), 604-12.

159. O'Halloran, J. A.; Barbosa, T. M.; Morrissey, J. P.; Kennedy, J.; O'Gara, F.; Dobson, A. D., Diversity and antimicrobial activity of Pseudovibrio spp. from Irish marine sponges. J Appl Microbiol 2011, 110, (6), 1495-508.

160. Schneemann, I.; Wiese, J.; Kunz, A. L.; Imhoff, J. F., Genetic approach for the fast discovery of phenazine producing bacteria. Mar. Drugs 2011, 9, (5), 772-89.

161. Penesyan, A.; Tebben, J.; Lee, M.; Thomas, T.; Kjelleberg, S.; Harder, T.; Egan, S., Identification of the antibacterial compound produced by the marine epiphytic bacterium Pseudovibrio sp. D323 and related sponge-associated bacteria. Mar. Drugs 2011, 9, (8), 1391-402.

162. Fiore, C. L.; Jarett, J. K.; Olson, N. D.; Lesser, M. P., Nitrogen fixation and nitrogen transformations in marine symbioses. Trends Microbiol 2010, 18, (10), 455-63.

163. Han, M.; Li, Z.; Zhang, F., The ammonia oxidizing and denitrifying prokaryotes associated with sponges from different sea areas. Microb Ecol 2013, 66, (2), 427-36.

164. Webster, N. S.; Negri, A. P.; Webb, R. I.; Hill, R. T., A spongin-boring alpha-proteobacterium is the etiological agent of disease in the Great Barrier Reef sponge Rhopaloeides odorabile. Mar Ecol Prog Ser 2002, 232, 305-309.

165. Sweet, M.; Bulling, M.; Cerrano, C., A novel sponge disease caused by a consortium of microorganisms. Coral Reefs 2015, 34, (3), 871-883.

166. Graca, A. P.; Bondoso, J.; Gaspar, H.; Xavier, J. R.; Monteiro, M. C.; de la Cruz, M.; Oves-Costales, D.; Vicente, F.; Lage, O. M., Antimicrobial activity of heterotrophic bacterial communities from the marine sponge Erylus discophorus (Astrophorida, Geodiidae). PLoS One 2013, 8, (11), e78992.

167. Whiteside, S. A.; Razvi, H.; Dave, S.; Reid, G.; Burton, J. P., The microbiome of the urinary tract--a role beyond infection. Nature reviews. Urology 2015, 12, (2), 81-90.

168. Vrieze, A.; Holleman, F.; Zoetendal, E. G.; de Vos, W. M.; Hoekstra, J. B. L.; Nieuwdorp, M., The environment within: how gut microbiota may influence metabolism and body composition. Diabetologia 2010, 53, (4), 606-613.

169. Sender, R.; Fuchs, S.; Milo, R., Are We Really Vastly Outnumbered? Revisiting the Ratio of Bacterial to Host Cells in Humans. Cell 2016, 164, (3), 337-40.

170. Arumugam, M.; Raes, J.; Pelletier, E.; Le Paslier, D.; Yamada, T.; Mende, D. R.; Fernandes, G. R.; Tap, J.; Bruls, T.; Batto, J. M.; Bertalan, M.; Borruel, N.; Casellas, F.; Fernandez, L.; Gautier, L.; Hansen, T.; Hattori, M.; Hayashi, T.; Kleerebezem, M.; Kurokawa, K.; Leclerc, M.; Levenez, F.; Manichanh, C.; Nielsen, H. B.; Nielsen, T.; Pons, N.; Poulain, J.; Qin, J.; Sicheritz-Ponten, T.; Tims, S.; Torrents, D.; Ugarte, E.; Zoetendal, E. G.; Wang, J.; Guarner, F.; Pedersen, O.; de Vos, W. M.; Brunak, S.; Dore, J.; Meta, H. I. T. C.; Antolin, M.; Artiguenave, F.; Blottiere, H. M.; Almeida, M.; Brechot, C.; Cara, C.; Chervaux, C.; Cultrone, A.; Delorme, C.; Denariaz, G.; Dervyn, R.; Foerstner, K. U.; Friss, C.; van de Guchte, M.; Guedon, E.; Haimet, F.; Huber, W.; van Hylckama-Vlieg, J.; Jamet, A.; Juste, C.; Kaci, G.; Knol, J.; Lakhdari, O.; Layec, S.; Le Roux, K.; Maguin, E.; Merieux, A.; Melo Minardi, R.; M'Rini, C.; Muller, J.; Oozeer, R.; Parkhill, J.; Renault, P.; Rescigno, M.; Sanchez, N.; Sunagawa, S.; Torrejon, A.; Turner, K.; Vandemeulebrouck, G.; Varela, E.; Winogradsky, Y.; Zeller, G.; Weissenbach, J.; Ehrlich, S. D.; Bork, P., Enterotypes of the human gut microbiome. Nature 2011, $473,(7346), 174-80$. 
171. Hoffmann, C.; Dollive, S.; Grunberg, S.; Chen, J.; Li, H.; Wu, G. D.; Lewis, J. D.; Bushman, F. D., Archaea and fungi of the human gut microbiome: correlations with diet and bacterial residents. PLoS One 2013, 8, (6), e66019.

172. Kim, B. S.; Kim, J. N.; Cerniglia, C. E., In Vitro Culture Conditions for Maintaining a Complex Population of Human Gastrointestinal Tract Microbiota. J. Biomed. Biotechnol. 2011.

173. Gibson, G. R.; Roberfroid, M. B., Dietary modulation of the human colonic microbiota: introducing the concept of prebiotics. J Nutr 1995, 125, (6), 1401-12.

174. Kelly, D.; Conway, S.; Aminov, R., Commensal gut bacteria: mechanisms of immune modulation. Trends Immunol 2005, 26, (6), 326-33.

175. Gill, S. R.; Pop, M.; Deboy, R. T.; Eckburg, P. B.; Turnbaugh, P. J.; Samuel, B. S.; Gordon, J. I.; Relman, D. A.; Fraser-Liggett, C. M.; Nelson, K. E., Metagenomic analysis of the human distal gut microbiome. Science 2006, 312, (5778), 1355-9.

176. Jernberg, C.; Lofmark, S.; Edlund, C.; Jansson, J. K., Long-term ecological impacts of antibiotic administration on the human intestinal microbiota. Isme $J$ 2007, 1, (1), 56-66.

177. Francino, M. P., Antibiotics and the Human Gut Microbiome: Dysbioses and Accumulation of Resistances. Front. Microbiol. 2015, 6, 1543.

178. Barbosa, T. M.; Levy, S. B., The impact of antibiotic use on resistance development and persistence. Drug Resist Updates 2000, 3, (5), 303-311.

179. Clemente, J. C.; Pehrsson, E. C.; Blaser, M. J.; Sandhu, K.; Gao, Z.; Wang, B.; Magris, M.; Hidalgo, G.; Contreras, M.; Noya-Alarcon, O.; Lander, O.; McDonald, J.; Cox, M.; Walter, J.; Oh, P. L.; Ruiz, J. F.; Rodriguez, S.; Shen, N.; Song, S. J.; Metcalf, J.; Knight, R.; Dantas, G.; Dominguez-Bello, M. G., The microbiome of uncontacted Amerindians. Science advances 2015, 1, (3).

180. Bartoloni, A.; Pallecchi, L.; Rodriguez, H.; Fernandez, C.; Mantella, A.; Bartalesi, F.; Strohmeyer, M.; Kristiansson, C.; Gotuzzo, E.; Paradisi, F.; Rossolini, G. M., Antibiotic resistance in a very remote Amazonas community. Int J Antimicrob Agents 2009, 33, (2), 125-129.

181. Bartoloni, A.; Bartalesi, F.; Mantella, A.; Dell'Amico, E.; Roselli, M.; Strohmeyer, M.; Barahona, H. G.; Barron, V. P.; Paradisi, F.; Rossolini, G. M., High prevalence of acquired antimicrobial resistance unrelated to heavy antimicrobial consumption. J Infect Dis 2004, 189, (7), 1291-1294.

182. Shoemaker, N. B.; Vlamakis, H.; Hayes, K.; Salyers, A. A., Evidence for extensive resistance gene transfer among Bacteroides spp. and among Bacteroides and other genera in the human colon. Appl Environ Microbiol 2001, 67, (2), 561-8.

183. Dortet, L.; Poirel, L.; Nordmann, P., Worldwide dissemination of the NDM-type carbapenemases in Gram-negative bacteria. BioMed research international 2014, 2014, 249856.

184. Deshpande, P.; Rodrigues, C.; Shetty, A.; Kapadia, F.; Hedge, A.; Soman, R., New Delhi Metallo-beta lactamase (NDM-1) in Enterobacteriaceae: treatment options with carbapenems compromised. $\mathrm{J}$. Assoc. Physicians India 2010, 58, 147-9.

185. Sommer, M. O.; Dantas, G.; Church, G. M., Functional characterization of the antibiotic resistance reservoir in the human microflora. Science 2009, 325, (5944), 1128-31.

186. van Reenen, C. A.; Dicks, L. M. T., Horizontal gene transfer amongst probiotic lactic acid bacteria and other intestinal microbiota: what are the possibilities? A review. Arch Microbiol 2011, 193, (3), 157168.

187. Smillie, C. S.; Smith, M. B.; Friedman, J.; Cordero, O. X.; David, L. A.; Alm, E. J., Ecology drives a global network of gene exchange connecting the human microbiome. Nature 2011, 480, (7376), 2414.

188. Liu, L.; Chen, X.; Skogerbo, G.; Zhang, P.; Chen, R.; He, S.; Huang, D. W., The human microbiome: a hot spot of microbial horizontal gene transfer. Genomics 2012, 100, (5), 265-70.

189. Donato, J. J.; Moe, L. A.; Converse, B. J.; Smart, K. D.; Berklein, F. C.; McManus, P. S.; Handelsman, J., Metagenomic analysis of apple orchard soil reveals antibiotic resistance genes encoding predicted bifunctional proteins. Appl Environ Microbiol 2010, 76, (13), 4396-401.

190. Yang, J.; Wang, C.; Shu, C.; Liu, L.; Geng, J.; Hu, S.; Feng, J., Marine sediment bacteria harbor antibiotic resistance genes highly similar to those found in human pathogens. Microb Ecol 2013, 65, (4), 975-981.

191. Segawa, T.; Takeuchi, N.; Rivera, A.; Yamada, A.; Yoshimura, Y.; Barcaza, G.; Shinbori, K.; Motoyama, H.; Kohshima, S.; Ushida, K., Distribution of antibiotic resistance genes in glacier environments. Environ. Microbiol. Rep. 2013, 5, (1), 127-34.

192. Forsberg, K. J.; Patel, S.; Gibson, M. K.; Lauber, C. L.; Knight, R.; Fierer, N.; Dantas, G., Bacterial phylogeny structures soil resistomes across habitats. Nature 2014, 509, (7502), 612-6.

193. Roberts, A. P.; Mullany, P., Oral biofilms: a reservoir of transferable, bacterial, antimicrobial resistance. Expert Rev. Anti Infect. Ther. 2010, 8, (12), 1441-50.

194. Heuer, H.; Schmitt, H.; Smalla, K., Antibiotic resistance gene spread due to manure application on agricultural fields. Curr Opin Microbiol 2011, 14, (3), 236-243.

195. Monk, B. C.; Goffeau, A., Outwitting multidrug resistance to antifungals. Science 2008, 321, (5887), 367-9.

196. Macinga, D. R.; Cook, G. M.; Poole, R. K.; Rather, P. N., Identification and characterization of aarF, a locus required for production of ubiquinone in Providencia stuartii and Escherichia coli and for expression of 2'-N-acetyltransferase in P. stuartii. J Bacteriol 1998, 180, (1), 128-35.

197. Sorek, R.; Cossart, P., Prokaryotic transcriptomics: a new view on regulation, physiology and pathogenicity. Nat Rev Genet 2010, 11, (1), 9-16.

198. Simon, C.; Daniel, R., Metagenomic analyses: past and future trends. Appl Environ Microbiol 2011, 77, (4), 1153-1161. 
199. Poretsky, R. S.; Hewson, I.; Sun, S. L.; Allen, A. E.; Zehr, J. P.; Moran, M. A., Comparative day/night metatranscriptomic analysis of microbial communities in the North Pacific subtropical gyre. Environ. Microbiol. 2009, 11, (6), 1358-1375.

200. Saleh-Lakha, S.; Miller, M.; Campbell, R. G.; Schneider, K.; Elahimanesh, P.; Hart, M. M.; Trevors, J. T., Microbial gene expression in soil: methods, applications and challenges. J Microbiol Meth 2005, 63, (1), 1-19.

201. Medema, M. H.; Blin, K.; Cimermancic, P.; de Jager, V.; Zakrzewski, P.; Fischbach, M. A.; Weber, T.; Takano, E.; Breitling, R., antiSMASH: rapid identification, annotation and analysis of secondary metabolite biosynthesis gene clusters in bacterial and fungal genome sequences. Nucleic Acids Res 2011, 39, (Web Server issue), W339-46.

202. Hu, Y.; Yang, X.; Qin, J.; Lu, N.; Cheng, G.; Wu, N.; Pan, Y.; Li, J.; Zhu, L.; Wang, X.; Meng, Z.; Zhao, F.; Liu, D.; Ma, J.; Qin, N.; Xiang, C.; Xiao, Y.; Li, L.; Yang, H.; Wang, J.; Yang, R.; Gao, G. F.; Zhu, B., Metagenome-wide analysis of antibiotic resistance genes in a large cohort of human gut microbiota. Nature communications 2013, 4, 2151.

203. Jechalke, S.; Schreiter, S.; Wolters, B.; Dealtry, S.; Heuer, H.; Smalla, K., Widespread dissemination of class 1 integron components in soils and related ecosystems as revealed by cultivation-independent analysis. Front. Microbiol. 2013, 4, 420.

204. Bhatnagar, I.; Kim, S. K., Pharmacologically prospective antibiotic agents and their sources: a marine microbial perspective. Environ Toxicol Pharmacol 2012, 34, (3), 631-43.

205. Reddy, B. V.; Kallifidas, D.; Kim, J. H.; Charlop-Powers, Z.; Feng, Z.; Brady, S. F., Natural product biosynthetic gene diversity in geographically distinct soil microbiomes. Appl Environ Microbiol 2012, $78,(10), 3744-52$.

206. Ayuso, A.; Clark, D.; Gonzalez, I.; Salazar, O.; Anderson, A.; Genilloud, O., A novel actinomycete strain de-replication approach based on the diversity of polyketide synthase and nonribosomal peptide synthetase biosynthetic pathways. Appl Microbiol Biotechnol 2005, 67, (6), 795-806.

207. Depardieu, F.; Podglajen, I.; Leclercq, R.; Collatz, E.; Courvalin, P., Modes and modulations of antibiotic resistance gene expression. Clin Microbiol Rev 2007, 20, (1), 79-114.

208. Jaurin, B.; Grundstrom, T., ampC cephalosporinase of Escherichia coli K-12 has a different evolutionary origin from that of beta-lactamases of the penicillinase type. Proc. Natl. Acad. Sci. USA 1981, 78, (8), 4897-901.

209. Kristiansson, E.; Fick, J.; Janzon, A.; Grabic, R.; Rutgersson, C.; Weijdegard, B.; Soderstrom, H.; Larsson, D. G., Pyrosequencing of antibiotic-contaminated river sediments reveals high levels of resistance and gene transfer elements. PLoS One 2011, 6, (2), e17038.

210. Reeves, A. Z.; Campbell, P. J.; Sultana, R.; Malik, S.; Murray, M.; Plikaytis, B. B.; Shinnick, T. M.; Posey, J. E., Aminoglycoside cross-resistance in Mycobacterium tuberculosis due to mutations in the $5^{\prime}$ untranslated region of whiB7. Antimicrob Agents Chemother 2013, 57, (4), 1857-65.

211. Yatsunenko, T.; Rey, F. E.; Manary, M. J.; Trehan, I.; Dominguez-Bello, M. G.; Contreras, M.; Magris, M.; Hidalgo, G.; Baldassano, R. N.; Anokhin, A. P.; Heath, A. C.; Warner, B.; Reeder, J.; Kuczynski, J.; Caporaso, J. G.; Lozupone, C. A.; Lauber, C.; Clemente, J. C.; Knights, D.; Knight, R.; Gordon, J. I., Human gut microbiome viewed across age and geography. Nature 2012, 486, (7402), 222-7.

212. Teuber, M.; Meile, L.; Schwarz, F., Acquired antibiotic resistance in lactic acid bacteria from food. Antonie Leeuwenhoek 1999, 76, (1-4), 115-37.

213. Fischer, A.; Liljander, A.; Kaspar, H.; Muriuki, C.; Fuxelius, H. H.; Bongcam-Rudloff, E.; de Villiers, E. P.; Huber, C. A.; Frey, J.; Daubenberger, C.; Bishop, R.; Younan, M.; Jores, J., Camel Streptococcus agalactiae populations are associated with specific disease complexes and acquired the tetracycline resistance gene tetM via a Tn916-like element. Vet. Res. 2013, 44, (1), 86.

214. Andersson, D. I.; Hughes, D., Antibiotic resistance and its cost: is it possible to reverse resistance? Nat. Rev. Microbiol. 2010, 8, (4), 260-71.

215. Levin, B. R.; Lipsitch, M.; Perrot, V.; Schrag, S.; Antia, R.; Simonsen, L.; Walker, N. M.; Stewart, F. M., The population genetics of antibiotic resistance. Clin Infect Dis 1997, 24 Suppl 1, S9-16.

216. Gullberg, E.; Cao, S.; Berg, O. G.; Ilback, C.; Sandegren, L.; Hughes, D.; Andersson, D. I., Selection of resistant bacteria at very low antibiotic concentrations. PLOS Pathog. 2011, 7, (7), e1002158.

217. Stewart, F. J.; Sharma, A. K.; Bryant, J. A.; Eppley, J. M.; DeLong, E. F., Community transcriptomics reveals universal patterns of protein sequence conservation in natural microbial communities. Genome Biol. 2011, 12, (3), R26.

218. Orsi, W. D.; Edgcomb, V. P.; Christman, G. D.; Biddle, J. F., Gene expression in the deep biosphere. Nature 2013, 499, (7457), 205-8.

219. Gifford, S. M.; Sharma, S.; Rinta-Kanto, J. M.; Moran, M. A., Quantitative analysis of a deeply sequenced marine microbial metatranscriptome. Isme J 2011, 5, (3), 461-472.

220. Aarestrup, F., Sustainable farming: Get pigs off antibiotics. Nature 2012, 486, (7404), 465-6.

221. van den Bogert, B.; Meijerink, M.; Zoetendal, E. G.; Wells, J. M.; Kleerebezem, M., Immunomodulatory properties of Streptococcus and Veillonella isolates from the human small intestine microbiota. PLoS One 2014, 9, (12), e114277.

222. Leimena, M. M.; Ramiro-Garcia, J.; Davids, M.; van den Bogert, B.; Smidt, H.; Smid, E. J.; Boekhorst, J.; Zoetendal, E. G.; Schaap, P. J.; Kleerebezem, M., A comprehensive metatranscriptome analysis pipeline and its validation using human small intestine microbiota datasets. BMC Genomics 2013, 14, 530.

223. Palmer, C.; Bik, E. M.; DiGiulio, D. B.; Relman, D. A.; Brown, P. O., Development of the human infant intestinal microbiota. PLOS Biol. 2007, 5, (7), e177.

224. Zoetendal, E. G.; Booijink, C. C.; Klaassens, E. S.; Heilig, H. G.; Kleerebezem, M.; Smidt, H.; de Vos, W. M., Isolation of RNA from bacterial samples of the human gastrointestinal tract. Nat. Protoc. 2006, $1,(2), 954-9$. 
225. Haenen, D.; Souza da Silva, C.; Zhang, J.; Koopmans, S. J.; Bosch, G.; Vervoort, J.; Gerrits, W. J.; Kemp, B.; Smidt, H.; Muller, M.; Hooiveld, G. J., Resistant starch induces catabolic but suppresses immune and cell division pathways and changes the microbiome in the proximal colon of male pigs. $J$ Nutr 2013, 143, (12), 1889-98.

226. Hugenholtz, F., Mouse gut microbiomics of short chain fatty acid metabolism and mucosal responses (Chapter 7). Doctoral dissertation 2015.

227. Ozturk, B.; de Jaeger, L.; Smidt, H.; Sipkema, D., Culture-dependent and independent approaches for identifying novel halogenases encoded by Crambe crambe (marine sponge) microbiota. Sci. Rep. 2013, 3, 2780.

228. Chomczynski, P.; Sacchi, N., Single-step method of RNA isolation by acid guanidinium thiocyanatephenol-chloroform extraction. Anal Biochem 1987, 162, (1), 156-9.

229. Kopylova, E.; Noe, L.; Touzet, H., SortMeRNA: fast and accurate filtering of ribosomal RNAs in metatranscriptomic data. Bioinformatics 2012, 28, (24), 3211-7.

230. Huang, Y.; Niu, B.; Gao, Y.; Fu, L.; Li, W., CD-HIT Suite: a web server for clustering and comparing biological sequences. Bioinformatics 2010, 26, (5), 680-2.

231. Zhang, Z.; Schwartz, S.; Wagner, L.; Miller, W., A greedy algorithm for aligning DNA sequences. J Comput Biol 2000, 7, (1-2), 203-14.

232. Clarebout, G.; Villers, C.; Leclercq, R., Macrolide resistance gene mreA of Streptococcus agalactiae encodes a flavokinase. Antimicrob Agents Chemother 2001, 45, (8), 2280-6.

233. Rida, S.; Caillet, J.; Alix, J. H., Amplification of a novel gene, sanA, abolishes a vancomycin-sensitive defect in Escherichia coli. J Bacteriol 1996, 178, (1), 94-102.

234. Clarke, K., Non-parametric multivariate analyses of changes in community structure. Aust $\mathrm{J}$ Ecol 1993, 18, 117-143.

235. Sipkema, D.; Blanch, H. W., Spatial distribution of bacteria associated with the marine sponge. Mar. Biol. 2010, 157, 627-638.

236. Hardoim, C. C.; Costa, R., Temporal dynamics of prokaryotic communities in the marine sponge Sarcotragus spinosulus. Mol Ecol 2014, 23, (12), 3097-112.

237. Reveillaud, J.; Maignien, L.; Eren, A. M.; Huber, J. A.; Apprill, A.; Sogin, M. L.; Vanreusel, A., Hostspecificity among abundant and rare taxa in the sponge microbiome. Isme J 2014, 8, (6), 1198-1209.

238. McFall-Ngai, M.; Hadfield, M. G.; Bosch, T. C.; Carey, H. V.; Domazet-Loso, T.; Douglas, A. E.; Dubilier, N.; Eberl, G.; Fukami, T.; Gilbert, S. F.; Hentschel, U.; King, N.; Kjelleberg, S.; Knoll, A. H.; Kremer, N.; Mazmanian, S. K.; Metcalf, J. L.; Nealson, K.; Pierce, N. E.; Rawls, J. F.; Reid, A.; Ruby, E. G.; Rumpho, M.; Sanders, J. G.; Tautz, D.; Wernegreen, J. J., Animals in a bacterial world, a new imperative for the life sciences. Proc Natl Acad Sci U S A 2013, 110, (9), 3229-36.

239. Thacker, R. W., Impacts of shading on sponge-Cyanobacteria symbioses: A comparison between hostspecific and generalist associations. Integr. Comp. Biol. 2005, 45, (2), 369-376.

240. Hoffmann, F.; Radax, R.; Woebken, D.; Holtappels, M.; Lavik, G.; Rapp, H. T.; Schlappy, M. L.; Schleper, C.; Kuypers, M. M. M., Complex nitrogen cycling in the sponge Geodia barretti. Environ. Microbiol. 2009, 11, (9), 2228-2243.

241. Hoppers, A.; Stoudenmire, J.; Wu, S.; Lopanik, N. B., Antibiotic activity and microbial community of the temperate sponge, Haliclona sp. J Appl Microbiol 2014, 118, (118), 419-430.

242. Mollica, A.; Locatelli, M.; Stefanucci, A.; Pinnen, F., Synthesis and Bioactivity of Secondary Metabolites from Marine Sponges Containing Dibrominated Indolic Systems. Molecules 2012, 17, (5), 6083-6099.

243. Proksch, P., Defensive roles for secondary metabolites from marine sponges and sponge-feeding nudibranchs. Toxicon 1994, 32, (6), 639-55.

244. Manivasagan, P.; Venkatesan, J.; Sivakumar, K.; Kim, S. K., Pharmaceutically active secondary metabolites of marine Actinobacteria. Microbiol Res 2014, 169, (4), 262-78.

245. Noro, J. C.; Kalaitzis, J. A.; Neilan, B. A., Bioactive Natural Products from Papua New Guinea Marine Sponges. Chem. Biodivers. 2012, 9, (10), 2077-2095.

246. Piel, J., Biosynthesis of polyketides by trans-AT polyketide synthases. Nat Prod Rep 2010, 27, (7), 996-1047.

247. Olson, J. B.; McCarthy, P. J., Associated bacterial communities of two deep-water sponges. Aquat Microb Ecol 2005, 39, (1), 47-55.

248. Hamady, M.; Walker, J. J.; Harris, J. K.; Gold, N. J.; Knight, R., Error-correcting barcoded primers for pyrosequencing hundreds of samples in multiplex. Nat. Methods 2008, 5, (3), 235-7.

249. van den Bogert, B.; de Vos, W. M.; Zoetendal, E. G.; Kleerebezem, M., Microarray analysis and barcoded pyrosequencing provide consistent microbial profiles depending on the source of human intestinal samples. Appl Environ Microbiol 2011, 77, (6), 2071-80.

250. Daims, H.; Bruhl, A.; Amann, R.; Schleifer, K. H.; Wagner, M., The domain-specific probe EUB338 is insufficient for the detection of all Bacteria: Development and evaluation of a more comprehensive probe set. Syst Appl Microbiol 1999, 22, (3), 434-444.

251. Caporaso, J. G.; Kuczynski, J.; Stombaugh, J.; Bittinger, K.; Bushman, F. D.; Costello, E. K.; Fierer, N.; Pena, A. G.; Goodrich, J. K.; Gordon, J. I.; Huttley, G. A.; Kelley, S. T.; Knights, D.; Koenig, J. E.; Ley, R. E.; Lozupone, C. A.; McDonald, D.; Muegge, B. D.; Pirrung, M.; Reeder, J.; Sevinsky, J. R.; Turnbaugh, P. J.; Walters, W. A.; Widmann, J.; Yatsunenko, T.; Zaneveld, J.; Knight, R., QIIME allows analysis of high-throughput community sequencing data. Nat. Methods 2010, 7, (5), 335-6.

252. Bragg, L.; Stone, G.; Imelfort, M.; Hugenholtz, P.; Tyson, G. W., Fast, accurate error-correction of amplicon pyrosequences using Acacia. Nat. Methods 2012, 9, (5), 425-426.

253. Edgar, R. C., Search and clustering orders of magnitude faster than BLAST. Bioinformatics 2010, 26, (19), 2460-1.

254. Smilauer, P.; Lepš, J., Multivariate analysis of ecological data using Canoco 5. Second edition. ed.; 2014; $\mathrm{p}$ xii, 362 pages. 
255. Clarke, K.; Gorley, R., PRIMER v6: User Manual/Tutorial, PRIMER-E. 2006

256. Ludwig, W.; Strunk, O.; Westram, R.; Richter, L.; Meier, H.; Yadhukumar; Buchner, A.; Lai, T.; Steppi, S.; Jobb, G.; Forster, W.; Brettske, I.; Gerber, S.; Ginhart, A. W.; Gross, O.; Grumann, S.; Hermann, S.; Jost, R.; Konig, A.; Liss, T.; Lussmann, R.; May, M.; Nonhoff, B.; Reichel, B.; Strehlow, R.; Stamatakis, A.; Stuckmann, N.; Vilbig, A.; Lenke, M.; Ludwig, T.; Bode, A.; Schleifer, K. H., ARB: a software environment for sequence data. Nucleic Acids Res 2004, 32, (4), 1363-71.

257. Jiang, H.; Dong, H.; Zhang, G.; Yu, B.; Chapman, L. R.; Fields, M. W., Microbial diversity in water and sediment of Lake Chaka, an athalassohaline lake in northwestern China. Appl Environ Microbiol 2006, 72, (6), 3832-45.

258. Schauer, M.; Massana, R.; Pedros-Alio, C., Spatial differences in bacterioplankton composition along the Catalan coast (NW Mediterranean) assessed by molecular fingerprinting. FEMS Microbiol Ecol 2000, 33, (1), 51-59.

259. Zhan, A. B.; Hulak, M.; Sylvester, F.; Huang, X.; Adebayo, A. A.; Abbott, C. L.; Adamowicz, S. J.; Heath, D. D.; Cristescu, M. E.; MacIsaac, H. J., High sensitivity of 454 pyrosequencing for detection of rare species in aquatic communities. Methods Ecol. Evol. 2013, 4, (6), 558-565.

260. Fieseler, L.; Horn, M.; Wagner, M.; Hentschel, U., Discovery of the novel candidate phylum "Poribacteria" in marine sponges. Appl Environ Microbiol 2004, 70, (6), 3724-32.

261. Siegl, A.; Kamke, J.; Hochmuth, T.; Piel, J.; Richter, M.; Liang, C.; Dandekar, T.; Hentschel, U., Single-cell genomics reveals the lifestyle of Poribacteria, a candidate phylum symbiotically associated with marine sponges. Isme $\mathrm{J}$ 2011, 5, (1), 61-70.

262. Kamke, J.; Rinke, C.; Schwientek, P.; Mavromatis, K.; Ivanova, N.; Sczyrba, A.; Woyke, T.; Hentschel, U., The Candidate Phylum Poribacteria by Single-Cell Genomics: New Insights into Phylogeny, CellCompartmentation, Eukaryote-Like Repeat Proteins, and Other Genomic Features. PLoS One 2014, 9, $(1)$.

263. Naim, M. A.; Morillo, J. A.; Sorensen, S. J.; Waleed, A. A.; Smidt, H.; Sipkema, D., Host-specific microbial communities in three sympatric North Sea sponges. FEMS Microbiol Ecol 2014, 90, (2), 390403.

264. Sanglier, J. J.; Wellington, E. M.; Behal, V.; Fiedler, H. P.; Ellouz Ghorbel, R.; Finance, C.; Hacene, M.; Kamoun, A.; Kelly, C.; Mercer, D. K.; et al., Novel bioactive compounds from Actinomycetes. Res Microbiol 1993, 144, (8), 661-3.

265. Yarza, P.; Yilmaz, P.; Pruesse, E.; Glockner, F. O.; Ludwig, W.; Schleifer, K. H.; Whitman, W. B.; Euzeby, J.; Amann, R.; Rossello-Mora, R., Uniting the classification of cultured and uncultured bacteria and archaea using 16S rRNA gene sequences. Nat. Rev. Microbiol. 2014, 12, (9), 635-45.

266. Hentschel, U.; Hopke, J.; Horn, M.; Friedrich, A. B.; Wagner, M.; Hacker, J.; Moore, B. S., Molecular evidence for a uniform microbial community in sponges from different oceans. Appl Environ Microbiol 2002, 68, (9), 4431-40.

267. Sunagawa, S.; Woodley, C. M.; Medina, M., Threatened corals provide underexplored microbial habitats. PLoS One 2010, 5, (3), e9554.

268. West, S. A.; Diggle, S. P.; Buckling, A.; Gardner, A.; Griffins, A. S., The social lives of microbes. Annu. Rev. Ecol. Evol. Syst. 2007, 38, 53-77.

269. Hardoim, C. C.; Cardinale, M.; Cucio, A. C.; Esteves, A. I.; Berg, G.; Xavier, J. R.; Cox, C. J.; Costa, R., Effects of sample handling and cultivation bias on the specificity of bacterial communities in keratose marine sponges. Front. Microbiol. 2014, 5, 611.

270. Schippers, K. J.; Sipkema, D.; Osinga, R.; Smidt, H.; Pomponi, S. A.; Martens, D. E.; Wijffels, R. H., Cultivation of sponges, sponge cells and symbionts: achievements and future prospects. Adv Mar Biol 2012, 62, 273-337.

271. Esteves, A. I.; Hardoim, C. C.; Xavier, J. R.; Goncalves, J. M.; Costa, R., Molecular richness and biotechnological potential of bacteria cultured from Irciniidae sponges in the north-east Atlantic. FEMS Microbiol Ecol 2013, 85, (3), 519-36.

272. Santos-Gandelman, J. F.; Santos, O. C.; Pontes, P. V.; Andrade, C. L.; Korenblum, E.; Muricy, G.; Giambiagi-Demarval, M.; Laport, M. S., Characterization of Cultivable Bacteria from Brazilian Sponges. Mar Biotechnol 2013, 15, (15), 667-76.

273. Arias, C. A.; Murray, B. E., Antibiotic-resistant bugs in the 21 st century--a clinical super-challenge. $N$ Engl J Med 2009, 360, (5), 439-43.

274. Lenski, R. E., Bacterial evolution and the cost of antibiotic resistance. Int Microbiol 1998, 1, (4), 26570.

275. Hogberg, L. D.; Heddini, A.; Cars, O., The global need for effective antibiotics: challenges and recent advances. Trends Pharmacol Sci 2010, 31, (11), 509-15.

276. Zhang, L.; An, R.; Wang, J.; Sun, N.; Zhang, S.; Hu, J.; Kuai, J., Exploring novel bioactive compounds from marine microbes. Curr Opin Microbiol 2005, 8, (3), 276-81.

277. Laport, M. S.; Santos, O. C. S.; Muricy, G., Marine Sponges: Potential Sources of New Antimicrobial Drugs. Curr Pharm Biotechnol 2009, 10, (1), 86-105.

278. August, P. R.; Tang, L.; Yoon, Y. J.; Ning, S.; Muller, R.; Yu, T. W.; Taylor, M.; Hoffmann, D.; Kim, C. G.; Zhang, X.; Hutchinson, C. R.; Floss, H. G., Biosynthesis of the ansamycin antibiotic rifamycin: deductions from the molecular analysis of the rif biosynthetic gene cluster of Amycolatopsis mediterranei S699. Chem. Biol. 1998, 5, (2), 69-79.

279. Schupp, T.; Toupet, C.; Engel, N.; Goff, S., Cloning and sequence analysis of the putative rifamycin polyketide synthase gene cluster from Amycolatopsis mediterranei. FEMS Microbiol Lett 1998, 159, (2), 201-7.

280. Kim, T. K.; Hewavitharana, A. K.; Shaw, P. N.; Fuerst, J. A., Discovery of a new source of rifamycin antibiotics in marine sponge Actinobacteria by phylogenetic prediction. Appl Environ Microbiol 2006, 72, (3), 2118-2125. 
281. Untergasser, A.; Nijveen, H.; Rao, X.; Bisseling, T.; Geurts, R.; Leunissen, J. A., Primer3Plus, an enhanced web interface to Primer3. Nucleic Acids Res 2007, 35, (Web Server issue), W71-4.

282. Zhu, W.; Lomsadze, A.; Borodovsky, M., Ab initio gene identification in metagenomic sequences. Nucleic Acids Res 2010, 38, (12), e132.

283. Altschul, S. F.; Gish, W.; Miller, W.; Myers, E. W.; Lipman, D. J., Basic local alignment search tool. J Mol Biol 1990, 215, (3), 403-10.

284. McArthur, A. G.; Waglechner, N.; Nizam, F.; Yan, A.; Azad, M. A.; Baylay, A. J.; Bhullar, K.; Canova, M. J.; De Pascale, G.; Ejim, L.; Kalan, L.; King, A. M.; Koteva, K.; Morar, M.; Mulvey, M. R.; O'Brien, J. S.; Pawlowski, A. C.; Piddock, L. J. V.; Spanogiannopoulos, P.; Sutherland, A. D.; Tang, I.; Taylor, P. L.; Thaker, M.; Wang, W. L.; Yan, M.; Yu, T.; Wright, G. D., The Comprehensive Antibiotic Resistance Database. Antimicrob Agents Chemother 2013, 57, (7), 3348-3357.

285. Mitchell, A.; Chang, H. Y.; Daugherty, L.; Fraser, M.; Hunter, S.; Lopez, R.; McAnulla, C.; McMenamin, C.; Nuka, G.; Pesseat, S.; Sangrador-Vegas, A.; Scheremetjew, M.; Rato, C.; Yong, S. Y.; Bateman, A.; Punta, M.; Attwood, T. K.; Sigrist, C. J.; Redaschi, N.; Rivoire, C.; Xenarios, I.; Kahn, D.; Guyot, D.; Bork, P.; Letunic, I.; Gough, J.; Oates, M.; Haft, D.; Huang, H.; Natale, D. A.; Wu, C. H.; Orengo, C.; Sillitoe, I.; Mi, H.; Thomas, P. D.; Finn, R. D., The InterPro protein families database: the classification resource after 15 years. Nucleic Acids Res 2015, 43, (Database issue), D213-21.

286. Sievers, F.; Wilm, A.; Dineen, D.; Gibson, T. J.; Karplus, K.; Li, W. Z.; Lopez, R.; McWilliam, H.; Remmert, M.; Soding, J.; Thompson, J. D.; Higgins, D. G., Fast, scalable generation of high-quality protein multiple sequence alignments using Clustal Omega. Mol. Syst. Biol. 2011, 7.

287. Siguier, P.; Perochon, J.; Lestrade, L.; Mahillon, J.; Chandler, M., ISfinder: the reference centre for bacterial insertion sequences. Nucleic Acids Res 2006, 34, (Database issue), D32-6.

288. Leplae, R.; Hebrant, A.; Wodak, S. J.; Toussaint, A., ACLAME: a CLAssification of Mobile genetic Elements. Nucleic Acids Res 2004, 32, (Database issue), D45-9.

289. Srivastava, A.; Singhal, N.; Goel, M.; Virdi, J. S.; Kumar, M., CBMAR: a comprehensive betalactamase molecular annotation resource. Database (Oxford) 2014, 2014, bau111.

290. Jacoby, G. A., Beta-lactamase nomenclature. Antimicrob Agents Chemother 2006, 50, (4), 1123-9.

291. Galimand, M.; Guiyoule, A.; Gerbaud, G.; Rasoamanana, B.; Chanteau, S.; Carniel, E.; Courvalin, P., Multidrug resistance in Yersinia pestis mediated by a transferable plasmid. N Engl J Med 1997, 337, (10), 677-80.

292. Lutz, R.; Bujard, H., Independent and tight regulation of transcriptional units in Escherichia coli via the LacR/O, the TetR/O and AraC/I1-I2 regulatory elements. Nucleic Acids Res 1997, 25, (6), 1203-10.

293. Sandegren, L., Selection of antibiotic resistance at very low antibiotic concentrations. Upsala J Med Sci 2014, 119, (2), 103-107.

294. Andersson, D. I.; Hughes, D., Evolution of antibiotic resistance at non-lethal drug concentrations. Drug Resist Updates 2012, 15, (3), 162-172.

295. Walsh, F.; Duffy, B., The culturable soil antibiotic resistome: a community of multi-drug resistant bacteria. PLoS One 2013, 8, (6), e65567.

296. Davies, J. E., Aminoglycosides: ancient and modern. J Antibiot (Tokyo) 2006, 59, (9), 529-32.

297. Wagner-Dobler, I.; Biebl, H., Environmental biology of the marine Roseobacter lineage. Annu Rev Microbiol 2006, 60, 255-80.

298. Aviles, E.; Rodriguez, A. D., Monamphilectine A, a potent antimalarial beta-lactam from marine sponge Hymeniacidon sp: isolation, structure, semisynthesis, and bioactivity. Org Lett 2010, 12, (22), 52903.

299. Allen, H. K.; Moe, L. A.; Rodbumrer, J.; Gaarder, A.; Handelsman, J., Functional metagenomics reveals diverse beta-lactamases in a remote Alaskan soil. Isme J 2009, 3, (2), 243-51.

300. Hall, B. G.; Barlow, M., Evolution of the serine beta-lactamases: past, present and future. Drug resistance updates : reviews and commentaries in antimicrobial and anticancer chemotherapy 2004, 7, (2), 111-23.

301. Garau, G.; Di Guilmi, A. M.; Hall, B. G., Structure-based phylogeny of the metallo-beta-lactamases. Antimicrob Agents Chemother 2005, 49, (7), 2778-2784.

302. Vogel, S., Current-Induced Flow through Living Sponges in Nature. Proc Natl Acad Sci U S A 1977, 74, (5), 2069-2071.

303. O'Halloran, J. A.; Barbosa, T. M.; Morrissey, J. P.; Kennedy, J.; Dobson, A. D.; O'Gara, F., Pseudovibrio axinellae sp. nov., isolated from an Irish marine sponge. Int J Syst Evol Microbiol 2013, 63, (Pt 1), 141-5.

304. Alex, A.; Antunes, A., Whole Genome Sequencing of the Symbiont Pseudovibrio sp. from the Intertidal Marine Sponge Polymastia penicillus Revealed a Gene Repertoire for Host-Switching Permissive Lifestyle. Genome Biol. Evol. 2015, 7, (11), 3022-32.

305. Romano, S.; Fernandez-Guerra, A.; Reen, F. J.; Glockner, F. O.; Crowley, S. P.; O'Sullivan, O.; Cotter, P. D.; Adams, C.; Dobson, A. D.; O'Gara, F., Comparative Genomic Analysis Reveals a Diverse Repertoire of Genes Involved in Prokaryote-Eukaryote Interactions within the Pseudovibrio Genus. Front. Microbiol. 2016, 7, 387.

306. Flemer, B.; Kennedy, J.; Margassery, L. M.; Morrissey, J. P.; O'Gara, F.; Dobson, A. D., Diversity and antimicrobial activities of microbes from two Irish marine sponges, Suberites carnosus and Leucosolenia sp. J Appl Microbiol 2012, 112, (2), 289-301.

307. Harrington, C.; Reen, F. J.; Mooij, M. J.; Stewart, F. A.; Chabot, J. B.; Guerra, A. F.; Glockner, F. O.; Nielsen, K. F.; Gram, L.; Dobson, A. D.; Adams, C.; O'Gara, F., Characterisation of non-autoinducing tropodithietic acid (TDA) production from marine sponge Pseudovibrio species. Mar. Drugs 2014, 12, (12), 5960-78.

308. Kennedy, J.; Baker, P.; Piper, C.; Cotter, P. D.; Walsh, M.; Mooij, M. J.; Bourke, M. B.; Rea, M. C.; O'Connor, P. M.; Ross, R. P.; Hill, C.; O'Gara, F.; Marchesi, J. R.; Dobson, A. D., Isolation and analysis 
of bacteria with antimicrobial activities from the marine sponge Haliclona simulans collected from Irish waters. Mar. Biotechnol. (N. Y.) 2009, 11, (3), 384-96.

309. Crowley, S. P.; O'Gara, F.; O'Sullivan, O.; Cotter, P. D.; Dobson, A. D., Marine Pseudovibrio sp. as a novel source of antimicrobials. Mar. Drugs 2014, 12, (12), 5916-29.

310. Koehorst, J. J.; van Dam, J. C. J.; van Heck, R. G. A.; Saccenti, E.; Martins dos Santos, V. A. P.; Schaap, P. J., High throughput functional comparison of 432 genome sequences of Pseudomonas using a semantic data framework. Sci. Rep. 2016, Under review.

311. Andrews, S., FASTQC: a quality control tool for high throughput sequence data. http://www.bioinformatics.bbsrc.ac.uk/projects/fastqc/ 2010.

312. Martin, M., Cutadapt removes adapter sequences from high-throughput sequencing reads. EMBNet.journal 2015, 21, (1), 10-12.

313. Coil, D.; Jospin, G.; Darling, A. E., A5-miseq: an updated pipeline to assemble microbial genomes from Illumina MiSeq data. Bioinformatics 2015, 31, (4), 587-9.

314. Zerbino, D. R.; Birney, E., Velvet: Algorithms for de novo short read assembly using de Bruijn graphs. Genome Res 2008, 18, (5), 821-829.

315. Walker, B. J.; Abeel, T.; Shea, T.; Priest, M.; Abouelliel, A.; Sakthikumar, S.; Cuomo, C. A.; Zeng, Q. D.; Wortman, J.; Young, S. K.; Earl, A. M., Pilon: An Integrated Tool for Comprehensive Microbial Variant Detection and Genome Assembly Improvement. PLoS One 2014, 9, (11).

316. Langmead, B.; Salzberg, S. L., Fast gapped-read alignment with Bowtie 2. Nat. Methods 2012, 9, (4), 357-9.

317. Li, H.; Handsaker, B.; Wysoker, A.; Fennell, T.; Ruan, J.; Homer, N.; Marth, G.; Abecasis, G.; Durbin, R.; Proc, G. P. D., The Sequence Alignment/Map format and SAMtools. Bioinformatics 2009, 25, (16), 2078-2079.

318. Quinlan, A. R.; Hall, I. M., BEDTools: a flexible suite of utilities for comparing genomic features. Bioinformatics 2010, 26, (6), 841-2.

319. Fiebig, A.; Pradella, S.; Petersen, J.; Pauker, O.; Michael, V.; Lunsdorf, H.; Goker, M.; Klenk, H. P.; Wagner-Dobler, I., Genome of the R-body producing marine alphaproteobacterium Labrenzia alexandrii type strain (DFL-11(T)). Stand Genomic Sci 2013, 7, (3), 413-426.

320. Blankenberg, D.; Von Kuster, G.; Coraor, N.; Ananda, G.; Lazarus, R.; Mangan, M.; Nekrutenko, A.; Taylor, J., Galaxy: a web-based genome analysis tool for experimentalists. Curr. Protoc. Mol. Biol. 2010, Chapter 19, Unit $19101-21$.

321. Lagesen, K.; Hallin, P.; Rodland, E. A.; Staerfeldt, H. H.; Rognes, T.; Ussery, D. W., RNAmmer: consistent and rapid annotation of ribosomal RNA genes. Nucleic Acids Res 2007, 35, (9), 3100-8.

322. Hyatt, D.; Chen, G. L.; Locascio, P. F.; Land, M. L.; Larimer, F. W.; Hauser, L. J., Prodigal: prokaryotic gene recognition and translation initiation site identification. BMC Bioinformatics 2010, 11, 119.

323. Claudel-Renard, C.; Chevalet, C.; Faraut, T.; Kahn, D., Enzyme-specific profiles for genome annotation: PRIAM. Nucleic Acids Res 2003, 31, (22), 6633-9.

324. Petersen, T. N.; Brunak, S.; von Heijne, G.; Nielsen, H., SignalP 4.0: discriminating signal peptides from transmembrane regions. Nat. Methods 2011, 8, (10), 785-6.

325. Krogh, A.; Larsson, B.; von Heijne, G.; Sonnhammer, E. L., Predicting transmembrane protein topology with a hidden Markov model: application to complete genomes. J Mol Biol 2001, 305, (3), 567-80.

326. Richter, M.; Rossello-Mora, R., Shifting the genomic gold standard for the prokaryotic species definition. Proc Natl Acad Sci U S A 2009, 106, (45), 19126-31.

327. Ekseth, O. K.; Kuiper, M.; Mironov, V., orthAgogue: an agile tool for the rapid prediction of orthology relations. Bioinformatics 2014, 30, (5), 734-6.

328. van Dongen, S., Graph Clustering by Flow Simulation. PhD Thesis 2000.

329. $\mathrm{R}$ development Core Team R: Language and environment for statistical computing, $\mathrm{R}$ Foundation for Statistical Computing. Retrieved from http//www.R-project.org: 2010.

330. Caspi, R.; Altman, T.; Billington, R.; Dreher, K.; Foerster, H.; Fulcher, C. A.; Holland, T. A.; Keseler, I. M.; Kothari, A.; Kubo, A.; Krummenacker, M.; Latendresse, M.; Mueller, L. A.; Ong, Q.; Paley, S.; Subhraveti, P.; Weaver, D. S.; Weerasinghe, D.; Zhang, P. F.; Karp, P. D., The MetaCyc database of metabolic pathways and enzymes and the BioCyc collection of Pathway/Genome Databases. Nucleic Acids Res 2014, 42, (D1), D459-D471.

331. Buchfink, B.; Xie, C.; Huson, D. H., Fast and sensitive protein alignment using DIAMOND. Nat. Methods 2015, 12, (1), 59-60.

332. Galperin, M. Y.; Makarova, K. S.; Wolf, Y. I.; Koonin, E. V., Expanded microbial genome coverage and improved protein family annotation in the COG database. Nucleic Acids Res 2015, 43, (Database issue), D261-9.

333. Jones, P.; Binns, D.; Chang, H. Y.; Fraser, M.; Li, W.; McAnulla, C.; McWilliam, H.; Maslen, J.; Mitchell, A.; Nuka, G.; Pesseat, S.; Quinn, A. F.; Sangrador-Vegas, A.; Scheremetjew, M.; Yong, S. Y.; Lopez, R.; Hunter, S., InterProScan 5: genome-scale protein function classification. Bioinformatics 2014, 30, (9), 1236-40.

334. Karpenahalli, M. R.; Lupas, A. N.; Soding, J., TPRpred: a tool for prediction of TPR-, PPR- and SEL1like repeats from protein sequences. BMC Bioinformatics 2007, 8, 2.

335. Medema, M. H.; Takano, E.; Breitling, R., Detecting sequence homology at the gene cluster level with MultiGeneBlast. Mol Biol Evol 2013, 30, (5), 1218-23.

336. Weber, T.; Blin, K.; Duddela, S.; Krug, D.; Kim, H. U.; Bruccoleri, R.; Lee, S. Y.; Fischbach, M. A.; Muller, R.; Wohlleben, W.; Breitling, R.; Takano, E.; Medema, M. H., antiSMASH 3.0-a comprehensive resource for the genome mining of biosynthetic gene clusters. Nucleic Acids Res 2015.

337. Ruger, H. J.; Hofle, M. G., Marine star-shaped-aggregate-forming bacteria: Agrobacterium atlanticum sp. nov.; Agrobacterium meteori sp. nov.; Agrobacterium ferrugineum sp. nov., nom. rev.; 
Agrobacterium gelatinovorum sp. nov., nom. rev.; and Agrobacterium stellulatum sp. nov., nom. rev. Int. J. Syst. Bacteriol. 1992, 42, (1), 133-43.

338. Donachie, S. P.; Bowman, J. P.; Alam, M., Nesiotobacter exalbescens gen. nov., sp nov., a moderately thermophilic alphaproteobacterium from an Hawaiian hypersaline lake. Int J Syst Evol Microbiol 2006, 56, 563-567.

339. Konstantinidis, K. T.; Tiedje, J. M., Genomic insights that advance the species definition for prokaryotes. Proc Natl Acad Sci U S A 2005, 102, (7), 2567-72.

340. Goris, J.; Konstantinidis, K. T.; Klappenbach, J. A.; Coenye, T.; Vandamme, P.; Tiedje, J. M., DNA-DNA hybridization values and their relationship to whole-genome sequence similarities. Int J Syst Evol Microbiol 2007, 57, (Pt 1), 81-91.

341. DeLong, E. F., The microbial ocean from genomes to biomes. Nature 2009, 459, (7244), 200-6.

342. Sun, S.; Chen, J.; Li, W.; Altintas, I.; Lin, A.; Peltier, S.; Stocks, K.; Allen, E. E.; Ellisman, M.; Grethe, J.; Wooley, J., Community cyberinfrastructure for Advanced Microbial Ecology Research and Analysis: the CAMERA resource. Nucleic Acids Res 2011, 39, (Database issue), D546-51.

343. Sharp, K. H.; Eam, B.; Faulkner, D. J.; Haygood, M. G., Vertical transmission of diverse microbes in the tropical sponge Corticium sp. Appl Environ Microbiol 2007, 73, (2), 622-9.

344. Schmitt, S.; Angermeier, H.; Schiller, R.; Lindquist, N.; Hentschel, U., Molecular microbial diversity survey of sponge reproductive stages and mechanistic insights into vertical transmission of microbial symbionts. Appl Environ Microbiol 2008, 74, (24), 7694-708.

345. Lee, O. O.; Chui, P. Y.; Wong, Y. H.; Pawlik, J. R.; Qian, P. Y., Evidence for vertical transmission of bacterial symbionts from adult to embryo in the Caribbean sponge Svenzea zeai. Appl Environ Microbiol 2009, 75, (19), 6147-56.

346. Sipkema, D.; de Caralt, S.; Morillo, J. A.; Al-Soud, W. A.; Sorensen, S. J.; Smidt, H.; Uriz, M. J., Similar sponge-associated bacteria can be acquired via both vertical and horizontal transmission. Environ. Microbiol. 2015.

347. Hendrickson, E. L.; Leigh, J. A., Roles of coenzyme F420-reducing hydrogenases and hydrogen- and F420-dependent methylenetetrahydromethanopterin dehydrogenases in reduction of F420 and production of hydrogen during methanogenesis. J Bacteriol 2008, 190, (14), 4818-21.

348. Mccormick, J. R. D.; Morton, G. O., Identity of Co-Synthetic Factor 1 of Streptomyces-Aureofaciens and Fragment Fo from Co-Enzyme F420 of Methanobacterium Species. J Am Chem Soc 1982, 104, (14), 4014-4015.

349. Peschke, U.; Schmidt, H.; Zhang, H. Z.; Piepersberg, W., Molecular characterization of the lincomycinproduction gene cluster of Streptomyces lincolnensis 78-11. Mol Microbiol 1995, 16, (6), 1137-56.

350. Ebert, S.; Rieger, P. G.; Knackmuss, H. J., Function of coenzyme F420 in aerobic catabolism of 2,4, 6trinitrophenol and 2,4-dinitrophenol by Nocardioides simplex FJ2-1A. J Bacteriol 1999, 181, (9), $2669-$ 74.

351. Stover, C. K.; Warrener, P.; VanDevanter, D. R.; Sherman, D. R.; Arain, T. M.; Langhorne, M. H.; Anderson, S. W.; Towell, J. A.; Yuan, Y.; McMurray, D. N.; Kreiswirth, B. N.; Barry, C. E.; Baker, W. R., A small-molecule nitroimidazopyran drug candidate for the treatment of tuberculosis. Nature 2000, 405, (6789), 962-6.

352. Purwantini, E.; Gillis, T. P.; Daniels, L., Presence of F420-dependent glucose-6-phosphate dehydrogenase in Mycobacterium and Nocardia species, but absence from Streptomyces and Corynebacterium species and methanogenic Archaea. FEMS Microbiol Lett 1997, 146, (1), 129-34.

353. Webster, N. S.; Thomas, T., The Sponge Hologenome. mBio 2016, 7, (2).

354. Antia, N. J.; Harrison, P. J.; Oliveira, L., The Role of Dissolved Organic Nitrogen in Phytoplankton Nutrition, Cell Biology and Ecology. Phycologia 1991, 30, (1), 1-89.

355. Su, J.; Jin, L. L.; Jiang, Q.; Sun, W.; Zhang, F. L.; Li, Z. Y., Phylogenetically Diverse ureC Genes and Their Expression Suggest the Urea Utilization by Bacterial Symbionts in Marine Sponge Xestospongia testudinaria. PLoS One 2013, 8, (5).

356. Wilkinson, C. R.; Fay, P., Nitrogen-Fixation in Coral-Reef Sponges with Symbiotic Cyanobacteria. Nature 1979, 279, (5713), 527-529.

357. Fan, L.; Liu, M.; Simister, R.; Webster, N. S.; Thomas, T., Marine microbial symbiosis heats up: the phylogenetic and functional response of a sponge holobiont to thermal stress. Isme J 2013, 7, (5), 991-1002.

358. Liu, M. Y.; Kjelleberg, S.; Thomas, T., Functional genomic analysis of an uncultured deltaproteobacterium in the sponge Cymbastela concentrica. Isme J 2011, 5, (3), 427-35.

359. Thomas, T.; Rusch, D.; DeMaere, M. Z.; Yung, P. Y.; Lewis, M.; Halpern, A.; Heidelberg, K. B.; Egan, S.; Steinberg, P. D.; Kjelleberg, S., Functional genomic signatures of sponge bacteria reveal unique and shared features of symbiosis. Isme J 2010, 4, (12), 1557-67.

360. Nguyen, M. T.; Liu, M.; Thomas, T., Ankyrin-repeat proteins from sponge symbionts modulate amoebal phagocytosis. Mol Ecol 2014, 23, (6), 1635-45.

361. Fan, L.; Reynolds, D.; Liu, M.; Stark, M.; Kjelleberg, S.; Webster, N. S.; Thomas, T., Functional equivalence and evolutionary convergence in complex communities of microbial sponge symbionts. Proc Natl Acad Sci U S A 2012, 109, (27), E1878-87.

362. Mittl, P. R.; Schneider-Brachert, W., Sel1-like repeat proteins in signal transduction. Cell Signal 2007, $19,(1), 20-31$.

363. Cerveny, L.; Straskova, A.; Dankova, V.; Hartlova, A.; Ceckova, M.; Staud, F.; Stulik, J., Tetratricopeptide repeat motifs in the world of bacterial pathogens: role in virulence mechanisms. Infect Immun 2013, 81, (3), 629-35.

364. Eicher, S. C.; Dehio, C., Bartonella entry mechanisms into mammalian host cells. Cell. Microbiol. 2012, 14, (8), 1166-1173. 
365. Coleman, S. A.; Minnick, M. F., Differential expression of the invasion-associated locus B (ialB) gene of Bartonella bacilliformis in response to environmental cues. Microb Pathog 2003, 34, (4), 179-86.

366. Tomich, M.; Planet, P. J.; Figurski, D. H., The tad locus: postcards from the widespread colonization island. Nat. Rev. Microbiol. 2007, 5, (5), 363-75.

367. Nasser, W.; Reverchon, S., New insights into the regulatory mechanisms of the LuxR family of quorum sensing regulators. Anal Bioanal Chem 2007, 387, (2), 381-390.

368. Miller, M. B.; Bassler, B. L., Quorum sensing in bacteria. Annu Rev Microbiol 2001, 55, 165-99.

369. Case, R. J.; Labbate, M.; Kjelleberg, S., AHL-driven quorum-sensing circuits: their frequency and function among the Proteobacteria. Isme J 2008, 2, (4), 345-349.

370. Subramoni, S.; Venturi, V., LuxR-family 'solos': bachelor sensors/regulators of signalling molecules. Microbiology 2009, 155, (Pt 5), 1377-85.

371. Dobretsov, S.; Teplitski, M.; Paul, V., Mini-review: quorum sensing in the marine environment and its relationship to biofouling. Biofouling 2009, 25, (5), 413-27.

372. Barnhart, M. M.; Chapman, M. R., Curli biogenesis and function. Annu Rev Microbiol 2006, 60, 13147.

373. Cornelis, G. R., The type III secretion injectisome. Nat. Rev. Microbiol. 2006, 4, (11), 811-25.

374. Coulthurst, S. J., The Type VI secretion system - a widespread and versatile cell targeting system. Res Microbiol 2013, 164, (6), 640-54.

375. Low, H. H.; Gubellini, F.; Rivera-Calzada, A.; Braun, N.; Connery, S.; Dujeancourt, A.; Lu, F.; Redzej, A.; Fronzes, R.; Orlova, E. V.; Waksman, G., Structure of a type IV secretion system. Nature 2014, 508, (7497), 550-3.

376. Biebl, H.; Pukall, R.; Lunsdorf, H.; Schulz, S.; Allgaier, M.; Tindall, B. J.; Wagner-Dobler, I., Description of Labrenzia alexandrii gen. nov., sp. nov., a novel alphaproteobacterium containing bacteriochlorophyll a, and a proposal for reclassification of Stappia aggregata as Labrenzia aggregata comb. nov., of Stappia marina as Labrenzia marina comb. nov. and of Stappia alba as Labrenzia alba comb. nov., and emended descriptions of the genera Pannonibacter, Stappia and Roseibium, and of the species Roseibium denhamense and Roseibium hamelinense. Int J Syst Evol Microbiol 2007, 57, (Pt 5), 1095-107.

377. Riley, M. A.; Wertz, J. E., Bacteriocins: evolution, ecology, and application. Annu Rev Microbiol 2002, 56, 117-37.

378. Balko, A. B., Characteristic, properties, prospect of application of bacteriocins. Mikrobiolohichnyi zhurnal 2012, 74, (6), 99-106.

379. Desriac, F.; Defer, D.; Bourgougnon, N.; Brillet, B.; Le Chevalier, P.; Fleury, Y., Bacteriocin as Weapons in the Marine Animal-Associated Bacteria Warfare: Inventory and Potential Applications as an Aquaculture Probiotic. Mar. Drugs 2010, 8, (4), 1153-1177.

380. Phelan, R. W.; Barret, M.; Cotter, P. D.; O'Connor, P. M.; Chen, R.; Morrissey, J. P.; Dobson, A. D.; O'Gara, F.; Barbosa, T. M., Subtilomycin: a new lantibiotic from Bacillus subtilis strain MMA7 isolated from the marine sponge Haliclona simulans. Mar. Drugs 2013, 11, (6), 1878-98.

381. Cane, D. E.; Ikeda, H., Exploration and mining of the bacterial terpenome. Acc. Chem. Res. 2012, 45, (3), 463-72.

382. Martin, D. G.; Slomp, G.; Mizsak, S.; Duchamp, D. J.; Chidester, C. G., The structure and absolute configuration of pentalenolactone (PA 132). Tetrahedron Lett 1970, (56), 4901-4.

383. Song, C.; Schmidt, R.; de Jager, V.; Krzyzanowska, D.; Jongedijk, E.; Cankar, K.; Beekwilder, J.; van Veen, A.; de Boer, W.; van Veen, J. A.; Garbeva, P., Exploring the genomic traits of fungus-feeding bacterial genus Collimonas. BMC Genomics 2015, 16, 1103.

384. Ding, L.; Goerls, H.; Dornblut, K.; Lin, W.; Maier, A.; Fiebig, H. H.; Hertweck, C., Bacaryolanes A-C, Rare Bacterial Caryolanes from a Mangrove Endophyte. J. Nat. Prod. 2015, 78, (12), 2963-7.

385. Epifanio, R. D.; Gabriel, R.; Martins, D. L.; Muricy, G., The sesterterpene variabilin as a fish-predation deterrent in the western Atlantic sponge Ircinia strobilina. J Chem Ecol 1999, 25, (10), 2247-2254.

386. Cheng, G.; Hu, Y.; Yin, Y.; Yang, X.; Xiang, C.; Wang, B.; Chen, Y.; Yang, F.; Lei, F.; Wu, N.; Lu, N.; $\mathrm{Li}$, J.; Chen, Q.; Li, L.; Zhu, B., Functional screening of antibiotic resistance genes from human gut microbiota reveals a novel gene fusion. FEMS Microbiol Lett 2012, 336, (1), 11-6.

387. Sommer, M. O. A., Advancing gut microbiome research using cultivation. Curr Opin Microbiol 2015, 27, 127-132.

388. Kim, B. S.; Kim, J. N.; Cerniglia, C. E., In vitro culture conditions for maintaining a complex population of human gastrointestinal tract microbiota. J. Biomed. Biotechnol. 2011, 2011, 838040.

389. Ma, L.; Kim, J.; Hatzenpichler, R.; Karymov, M. A.; Hubert, N.; Hanan, I. M.; Chang, E. B.; Ismagilov, R. F., Gene-targeted microfluidic cultivation validated by isolation of a gut bacterium listed in Human Microbiome Project's Most Wanted taxa. Proc Natl Acad Sci U S A 2014, 111, (27), 9768-9773.

390. Rettedal, E. A.; Gumpert, H.; Sommer, M. O., Cultivation-based multiplex phenotyping of human gut microbiota allows targeted recovery of previously uncultured bacteria. Nature communications 2014, $5,4714$.

391. Fodor, A. A.; DeSantis, T. Z.; Wylie, K. M.; Badger, J. H.; Ye, Y.; Hepburn, T.; Hu, P.; Sodergren, E.; Liolios, K.; Huot-Creasy, H.; Birren, B. W.; Earl, A. M., The "most wanted" taxa from the human microbiome for whole genome sequencing. PLoS One 2012, 7, (7), e41294.

392. de Smet, A. M. G. A.; Bonten, M. J. M.; Kluytmans, J. A. J. W., For whom should we use selective decontamination of the digestive tract? Curr Opin Infect Dis 2012, 25, (2), 211-217.

393. de Jonge, E.; Schultz, M. J.; Spanjaard, L.; Bossuyt, P. M.; Vroom, M. B.; Dankert, J.; Kesecioglu, J., Effects of selective decontamination of digestive tract on mortality and acquisition of resistant bacteria in intensive care: a randomised controlled trial. Lancet 2003, 362, (9389), 1011-6.

394. de Smet, A. M.; Kluytmans, J. A.; Cooper, B. S.; Mascini, E. M.; Benus, R. F.; van der Werf, T. S.; van der Hoeven, J. G.; Pickkers, P.; Bogaers-Hofman, D.; van der Meer, N. J.; Bernards, A. T.; Kuijper, E. 
J.; Joore, J. C.; Leverstein-van Hall, M. A.; Bindels, A. J.; Jansz, A. R.; Wesselink, R. M.; de Jongh, B. M.; Dennesen, P. J.; van Asselt, G. J.; te Velde, L. F.; Frenay, I. H.; Kaasjager, K.; Bosch, F. H.; van Iterson, M.; Thijsen, S. F.; Kluge, G. H.; Pauw, W.; de Vries, J. W.; Kaan, J. A.; Arends, J. P.; Aarts, L. P.; Sturm, P. D.; Harinck, H. I.; Voss, A.; Uijtendaal, E. V.; Blok, H. E.; Thieme Groen, E. S.; Pouw, M. E.; Kalkman, C. J.; Bonten, M. J., Decontamination of the digestive tract and oropharynx in ICU patients. N Engl J Med 2009, 360, (1), 20-31.

395. Daneman, N.; Sarwar, S.; Fowler, R. A.; Cuthbertson, B. H.; Su, D. C. S. G., Effect of selective decontamination on antimicrobial resistance in intensive care units: a systematic review and metaanalysis. Lancet Infect. Dis. 2013, 13, (4), 328-41.

396. Bülow, E., The Human Gut Microbiota as a Reservoir for Antimicrobial Resistance Genes (Chapter 3). Doctoral dissertation 2015.

397. Stams, A. J.; Van Dijk, J. B.; Dijkema, C.; Plugge, C. M., Growth of syntrophic propionate-oxidizing bacteria with fumarate in the absence of methanogenic bacteria. Appl Environ Microbiol 1993, 59, (4), 1114-9.

398. Salonen, A.; Nikkila, J.; Jalanka-Tuovinen, J.; Immonen, O.; Rajilic-Stojanovic, M.; Kekkonen, R. A.; Palva, A.; de Vos, W. M., Comparative analysis of fecal DNA extraction methods with phylogenetic microarray: Effective recovery of bacterial and archaeal DNA using mechanical cell lysis. J Microbiol Methods 2010, 81, (2), 127-134.

399. Tian, L.; Scholte, J.; Borewicz, K.; Bogert, B. V.; Smidt, H.; Scheurink, A. J.; Gruppen, H.; Schols, H. A., Effects of pectin supplementation on the fermentation patterns of different structural carbohydrates in rats. Mol. Nutr. Food Res. 2016.

400. Ramiro-Garcia, J.; Hermes, G. D. A.; Giatsis, C.; Sipkema, D.; Zoetendal, E. G.; Schaap, P. J.; Smidt, H., NG-Tax, a highly accurate and validated pipeline for analysis of $16 \mathrm{~S}$ rRNA amplicons from complex biomes. F1000Res 2016, 5, 1791.

401. Quast, C.; Pruesse, E.; Yilmaz, P.; Gerken, J.; Schweer, T.; Yarza, P.; Peplies, J.; Glockner, F. O., The SILVA ribosomal RNA gene database project: improved data processing and web-based tools. Nucleic Acids Res 2013, 41, (Database issue), D590-6.

402. Seo, B.; Yoo, J. E.; Lee, Y. M.; Ko, G., Sellimonas intestinalis gen. nov., sp. nov., Isolated from Human Faeces. Int J Syst Evol Microbiol 2015.

403. Raes, J., Microbiome-based companion diagnostics: no longer science fiction? Gut 2016, 65, (6), 896U207.

404. Falcon, L. I.; Magallon, S.; Castillo, A., Dating the cyanobacterial ancestor of the chloroplast. Isme J 2010, 4, (6), 777-83.

405. Deak, E.; Szabo, I.; Kalmaczhelyi, A.; Gal, Z.; Barabas, G.; Penyige, A., Membrane-bound and extracellular beta-lactamase production with developmental regulation in Streptomyces griseus NRRL B-2682. Microbiology 1998, 144 ( Pt 8), 2169-77.

406. Blechschmidt, B.; Borneleit, P.; Kleber, H. P., Purification and characterization of an extracellular betalactamase produced by Acinetobacter calcoaceticus. J. Gen. Microbiol. 1992, 138, (6), 1197-202.

407. Al Naiemi, N.; Heddema, E. R.; Bart, A.; de Jonge, E.; Vandenbroucke-Grauls, C. M.; Savelkoul, P. H.; Duim, B., Emergence of multidrug-resistant Gram-negative bacteria during selective decontamination of the digestive tract on an intensive care unit. J Antimicrob Chemother 2006, 58, (4), 853-6.

408. Wolf, M.; Muller, T.; Dandekar, T.; Pollack, J. D., Phylogeny of Firmicutes with special reference to Mycoplasma (Mollicutes) as inferred from phosphoglycerate kinase amino acid sequence data. Int J Syst Evol Microbiol 2004, 54, 871-875.

409. Pillai, D. R.; McGeer, A.; Low, D. E., New Delhi metallo-beta-lactamase-1 in Enterobacteriaceae: emerging resistance. CMAJ 2011, 183, (1), 59-64.

410. Casey, G., Antibiotics and the rise of superbugs. Nurs. N. Z. 2012, 18, (10), 20-4.

411. UK Government, Review on antimicrobial resistance: tackling drug-resistant infections globally. http://amr-review.org/ 2016.

412. Raviglione, M., XDR-TB: entering the post-antibiotic era? Int. J. Tuberc. Lung Dis. 2006, 10, (11), 1185-7.

413. Falagas, M. E.; Bliziotis, I. A., Pandrug-resistant Gram-negative bacteria: the dawn of the postantibiotic era? Int J Antimicrob Agents 2007, 29, (6), 630-6.

414. Haas, B. J.; Chin, M.; Nusbaum, C.; Birren, B. W.; Livny, J., How deep is deep enough for RNA-Seq profiling of bacterial transcriptomes? BMC Genomics 2012, 13.

415. Sekirov, I.; Russell, S. L.; Antunes, L. C.; Finlay, B. B., Gut microbiota in health and disease. Physiol Rev 2010, 90, (3), 859-904.

416. Nielsen, H. B.; Almeida, M.; Juncker, A. S.; Rasmussen, S.; Li, J. H.; Sunagawa, S.; Plichta, D. R.; Gautier, L.; Pedersen, A. G.; Le Chatelier, E.; Pelletier, E.; Bonde, I.; Nielsen, T.; Manichanh, C.; Arumugam, M.; Batto, J. M.; dos Santos, M. B. Q.; Blom, N.; Borruel, N.; Burgdorf, K. S.; Boumezbeur, F.; Casellas, F.; Dore, J.; Dworzynski, P.; Guarner, F.; Hansen, T.; Hildebrand, F.; Kaas, R. S.; Kennedy, S.; Kristiansen, K.; Kultima, J. R.; Leonard, P.; Levenez, F.; Lund, O.; Moumen, B.; Le Paslier, D.; Pons, N.; Pedersen, O.; Prifti, E.; Qin, J. J.; Raes, J.; Sorensen, S.; Tap, J.; Tims, S.; Ussery, D. W.; Yamada, T.; Renault, P.; Sicheritz-Ponten, T.; Bork, P.; Wang, J.; Brunak, S.; Ehrlich, S. D.; Consortium, M., Identification and assembly of genomes and genetic elements in complex metagenomic samples without using reference genomes. Nat Biotechnol 2014, 32, (8), 822-828.

417. Qin, J.; Li, R.; Raes, J.; Arumugam, M.; Burgdorf, K. S.; Manichanh, C.; Nielsen, T.; Pons, N.; Levenez, F.; Yamada, T.; Mende, D. R.; Li, J.; Xu, J.; Li, S.; Li, D.; Cao, J.; Wang, B.; Liang, H.; Zheng, H.; Xie, Y.; Tap, J.; Lepage, P.; Bertalan, M.; Batto, J. M.; Hansen, T.; Le Paslier, D.; Linneberg, A.; Nielsen, H. B.; Pelletier, E.; Renault, P.; Sicheritz-Ponten, T.; Turner, K.; Zhu, H.; Yu, C.; Li, S.; Jian, M.; Zhou, Y.; Li, Y.; Zhang, X.; Li, S.; Qin, N.; Yang, H.; Wang, J.; Brunak, S.; Dore, J.; Guarner, F.; Kristiansen, K.; Pedersen, O.; Parkhill, J.; Weissenbach, J.; Meta, H. I. T. C.; Bork, P.; 
Ehrlich, S. D.; Wang, J., A human gut microbial gene catalogue established by metagenomic sequencing. Nature 2010, 464, (7285), 59-65.

418. Ni, J. J.; Yan, Q. Y.; Yu, Y. H., How much metagenomic sequencing is enough to achieve a given goal? Sci. Rep. 2013, 3.

419. Zhu, Y. G.; Johnson, T. A.; Su, J. Q.; Qiao, M.; Guo, G. X.; Stedtfeld, R. D.; Hashsham, S. A.; Tiedje, J. M., Diverse and abundant antibiotic resistance genes in Chinese swine farms. Proc Natl Acad Sci U S A 2013, 110, (9), 3435-40.

420. Mercer, T. R.; Gerhardt, D. J.; Dinger, M. E.; Crawford, J.; Trapnell, C.; Jeddeloh, J. A.; Mattick, J. S.; Rinn, J. L., Targeted RNA sequencing reveals the deep complexity of the human transcriptome. Nat Biotechnol 2012, 30, (1), 99-104.

421. Schmieder, R. A., A Framework for Identifying Antibiotic Resistance in the Human Microbiome. Doctoral dissertation 2012.

422. Leekha, S.; Terrell, C. L.; Edson, R. S., General principles of antimicrobial therapy. Mayo Clin Proc 2011, 86, (2), 156-67.

423. Sarno, R.; Ha, H.; Weinsetel, N.; Tolmasky, M. E., Inhibition of aminoglycoside 6'-N-acetyltransferase type Ib-mediated amikacin resistance by antisense oligodeoxynucleotides. Antimicrob Agents Chemother 2003, 47, (10), 3296-304.

424. Boehr, D. D.; Draker, K. A.; Koteva, K.; Bains, M.; Hancock, R. E.; Wright, G. D., Broad-spectrum peptide inhibitors of aminoglycoside antibiotic resistance enzymes. Chem. Biol. 2003, 10, (2), 189-96.

425. Benveniste, R.; Davies, J., Aminoglycoside antibiotic-inactivating enzymes in actinomycetes similar to those present in clinical isolates of antibiotic-resistant bacteria. Proc Natl Acad Sci U S A 1973, 70, (8), 2276-80.

426. Sommer, M. O.; Church, G. M.; Dantas, G., The human microbiome harbors a diverse reservoir of antibiotic resistance genes. Virulence 2010, 1, (4), 299-303.

427. Riesenfeld, C. S.; Goodman, R. M.; Handelsman, J., Uncultured soil bacteria are a reservoir of new antibiotic resistance genes. Environ. Microbiol. 2004, 6, (9), 981-9.

428. Lang, K. S.; Anderson, J. M.; Schwarz, S.; Williamson, L.; Handelsman, J.; Singer, R. S., Novel florfenicol and chloramphenicol resistance gene discovered in Alaskan soil by using functional metagenomics. Appl Environ Microbiol 2010, 76, (15), 5321-6.

429. Van Soest, R. W. M.; Boury-Esnault, N.; Vacelet, J.; Dohrmann, M.; Erpenbeck, D.; De Voogd, N. J.; Santodomingo, N.; Vanhoorne, B.; Kelly, M.; Hooper, J. N. A., Global Diversity of Sponges (Porifera). PLOS One 2012, 7, (4).

430. Hannig, G.; Makrides, S. C., Strategies for optimizing heterologous protein expression in Escherichia coli. Trends Biotechnol 1998, 16, (2), 54-60.

431. Mullany, P., Functional metagenomics for the investigation of antibiotic resistance. Virulence 2014, 5, (3), 443-447.

432. Biver, S.; Steels, S.; Portetelle, D.; Vandenbol, M., Bacillus subtilis as a Tool for Screening Soil Metagenomic Libraries for Antimicrobial Activities. J Microbiol Biotechnol 2013, 23, (6), 850-855.

433. Noreen, N.; Hooi, W. Y.; Baradaran, A.; Rosfarizan, M.; Sieo, C. C.; Rosli, M. I.; Yusoff, K.; Raha, A. R., Lactococcus lactis M4, a potential host for the expression of heterologous proteins. Microbial cell factories 2011, 10, 28.

434. Barquist, L.; Boinett, C. J.; Cain, A. K., Approaches to querying bacterial genomes with transposoninsertion sequencing. RNA Biol. 2013, 10, (7), 1161-9.

435. Martinez, J. L.; Coque, T. M.; Baquero, F., What is a resistance gene? Ranking risk in resistomes. Nature Reviews Microbiology 2015, 13, (2), 116-123.

436. Clinical and Laboratory Standards Institute, Performance Standards for Antimicrobial Susceptibility Testing. Twenty-Fifth Informational Supplement M100-S25. Clinical and Laboratory Standards Institute 2015.

437. Kassim, A.; Omuse, G.; Premji, Z.; Revathi, G., Comparison of Clinical Laboratory Standards Institute and European Committee on Antimicrobial Susceptibility Testing guidelines for the interpretation of antibiotic susceptibility at a University teaching hospital in Nairobi, Kenya: a cross-sectional study. Ann. Clin. Microbiol. Antimicrob. 2016, 15.

438. Kahlmeter, G.; Brown, D. F.; Goldstein, F. W.; MacGowan, A. P.; Mouton, J. W.; Osterlund, A.; Rodloff, A.; Steinbakk, M.; Urbaskova, P.; Vatopoulos, A., European harmonization of MIC breakpoints for antimicrobial susceptibility testing of bacteria. J Antimicrob Chemother 2003, 52, (2), 145-8.

439. Wright, G. D., Antibiotic resistance in the environment: a link to the clinic? Curr Opin Microbiol 2010, $13,(5), 589-94$.

440. Humeniuk, C.; Arlet, G.; Gautier, V.; Grimont, P.; Labia, R.; Philippon, A., beta-lactamases of Kluyvera ascorbata, probable progenitors of some plasmid-encoded CTX-M types. Antimicrob Agents Chemother 2002, 46, (9), 3045-3049.

441. Vary, P. S.; Biedendieck, R.; Fuerch, T.; Meinhardt, F.; Rohde, M.; Deckwer, W. D.; Jahn, D., Bacillus megaterium--from simple soil bacterium to industrial protein production host. Appl Microbiol Biotechnol 2007, 76, (5), 957-67.

442. Simister, R. L.; Deines, P.; Botte, E. S.; Webster, N. S.; Taylor, M. W., Sponge-specific clusters revisited: a comprehensive phylogeny of sponge-associated microorganisms. Environ. Microbiol. 2012, $14,(2), 517-524$.

443. Diaz, M. C.; Rutzler, K., Sponges: An essential component of Caribbean coral reefs. Bull Mar Sci 2001, $69,(2), 535-546$.

444. Taylor, M. W.; Tsai, P.; Simister, R. L.; Deines, P.; Botte, E.; Ericson, G.; Schmitt, S.; Webster, N. S., 'Sponge-specific' bacteria are widespread (but rare) in diverse marine environments. Isme J 2013, 7 , (2), 438-43. 
445. Baquero, F.; Martinez, J. L.; Canton, R., Antibiotics and antibiotic resistance in water environments. Curr Opin Biotechnol 2008, 19, (3), 260-265.

446. Nelson, E. C.; Segal, H.; Elisha, B. G., Outer membrane protein alterations and bla(TEM-1) variants: their role in beta-lactam resistance in Klebsiella pneumoniae. J Antimicrob Chemother 2003, 52, (6), 899-903.

447. Zhang, Y.; Zhou, H.; Shen, X. Q.; Shen, P.; Yu, Y. S.; Li, L. J., Plasmid-borne armA methylase gene, together with bla(CTX-M-15) and bla(TEM-1), in a Klebsiella oxytoca isolate from China. J Med Microbiol 2008, 57, (10), 1273-1276.

448. Sondergaard, A.; San Millan, A.; Santos-Lopez, A.; Nielsen, S. M.; Gonzalez-Zorn, B.; NorskovLauritsen, N., Molecular organization of small plasmids bearing blaTEM-1 and conferring resistance to beta-lactams in Haemophilus influenzae. Antimicrob Agents Chemother 2012, 56, (9), 4958-60.

449. Valdezate, S.; Garrido, N.; Carrasco, G.; Villalon, P.; Medina-Pascual, M. J.; Saez-Nieto, J. A., Resistance gene pool to co-trimoxazole in non-susceptible Nocardia strains. Front. Microbiol. 2015, 6, 376.

450. Datta, N.; Kontomichalou, P., Penicillinase synthesis controlled by infectious R factors in Enterobacteriaceae. Nature 1965, 208, (5007), 239-41.

451. Carattoli, A., Plasmid-mediated antimicrobial resistance in Salmonella enterica. Curr Issues Mol Biol 2003, 5, (4), 113-22.

452. Gomez-Lus, R., Evolution of bacterial resistance to antibiotics during the last three decades. Int Microbiol 1998, 1, (4), 279-84.

453. White, R. A.; Chan, A. M.; Gavelis, G. S.; Leander, B. S.; Brady, A. L.; Slater, G. F.; Lim, D. S. S.; Suttle, C. A., Metagenomic Analysis Suggests Modern Freshwater Microbialites Harbor a Distinct Core Microbial Community. Front. Microbiol. 2016, 6.

454. Barros, J.; Igrejas, G.; Andrade, M.; Radhouani, H.; Lopez, M.; Torres, C.; Poeta, P., Gilthead seabream (Sparus aurata) carrying antibiotic resistant enterococci. A potential bioindicator of marine contamination? Mar Pollut Bull 2011, 62, (6), 1245-8.

455. Foti, M.; Giacopello, C.; Bottari, T.; Fisichella, V.; Rinaldo, D.; Mammina, C., Antibiotic Resistance of Gram-negative isolates from loggerhead sea turtles (Caretta caretta) in the central Mediterranean Sea. Mar Pollut Bull 2009, 58, (9), 1363-6.

456. Ouyang, W. Y.; Huang, F. Y.; Zhao, Y.; Li, H.; Su, J. Q., Increased levels of antibiotic resistance in urban stream of Jiulongjiang River, China. Appl Microbiol Biotechnol 2015, 99, (13), 5697-707.

457. Port, J. A.; Wallace, J. C.; Griffith, W. C.; Faustman, E. M., Metagenomic profiling of microbial composition and antibiotic resistance determinants in Puget Sound. PLoS One 2012, 7, (10), e48000.

458. Marti, E.; Jofre, J.; Balcazar, J. L., Prevalence of antibiotic resistance genes and bacterial community composition in a river influenced by a wastewater treatment plant. PLoS One 2013, 8, (10), e78906.

459. Rizzo, L.; Manaia, C.; Merlin, C.; Schwartz, T.; Dagot, C.; Ploy, M. C.; Michael, I.; Fatta-Kassinos, D., Urban wastewater treatment plants as hotspots for antibiotic resistant bacteria and genes spread into the environment: A review. Sci Total Environ 2013, 447, 345-360.

460. Martiny, A. C.; Martiny, J. B.; Weihe, C.; Field, A.; Ellis, J. C., Functional metagenomics reveals previously unrecognized diversity of antibiotic resistance genes in gulls. Front. Microbiol. 2011, 2, 238.

461. Blanco, G.; Lemus, J. A.; Grande, J., Microbial pollution in wildlife: Linking agricultural manuring and bacterial antibiotic resistance in red-billed choughs. Environ Res 2009, 109, (4), 405-12.

462. Hasan, B.; Melhus, A.; Sandegren, L.; Alam, M.; Olsen, B., The gull (Chroicocephalus brunnicephalus) as an environmental bioindicator and reservoir for antibiotic resistance on the coastlines of the Bay of Bengal. Microb Drug Resist 2014, 20, (5), 466-71.

463. Sapkota, A. R.; Curriero, F. C.; Gibson, K. E.; Schwab, K. J., Antibiotic-resistant enterococci and fecal indicators in surface water and groundwater impacted by a concentrated swine feeding operation. Environ Health Perspect 2007, 115, (7), 1040-1045.

464. Byrne-Bailey, K. G.; Gaze, W. H.; Kay, P.; Boxall, A. B.; Hawkey, P. M.; Wellington, E. M., Prevalence of sulfonamide resistance genes in bacterial isolates from manured agricultural soils and pig slurry in the United Kingdom. Antimicrob Agents Chemother 2009, 53, (2), 696-702.

465. Peak, N.; Knapp, C. W.; Yang, R. K.; Hanfelt, M. M.; Smith, M. S.; Aga, D. S.; Graham, D. W., Abundance of six tetracycline resistance genes in wastewater lagoons at cattle feedlots with different antibiotic use strategies. Environ. Microbiol. 2007, 9, (1), 143-151.

466. Andrade, V. D.; Zampieri, B. D.; Ballesteros, E. R.; Pinto, A. B.; de Oliveira, A. J. F. C., Densities and antimicrobial resistance of Escherichia coli isolated from marine waters and beach sands. Environ Monit Assess 2015, 187, (6).

467. Cabello, F. C., Heavy use of prophylactic antibiotics in aquaculture: a growing problem for human and animal health and for the environment. Environ. Microbiol. 2006, 8, (7), 1137-1144.

468. Chuah, L. O.; Effarizah, M. E.; Goni, A. M.; Rusul, G., Antibiotic Application and Emergence of Multiple Antibiotic Resistance (MAR) in Global Catfish Aquaculture. Current environmental health reports 2016.

469. Muziasari, W. I.; Parnanen, K.; Johnson, T. A.; Lyra, C.; Karkman, A.; Stedtfeld, R. D.; Tamminen, M.; Tiedje, J. M.; Virta, M., Aquaculture changes the profile of antibiotic resistance and mobile genetic element associated genes in Baltic Sea sediments. FEMS Microbiol Ecol 2016, 92, (4).

470. Mirand, C. D.; Zemelman, R., Antimicrobial multiresistance in bacteria isolated from freshwater Chilean salmon farms. Sci Total Environ 2002, 293, (1-3), 207-18.

471. Na, G.; Zhang, W.; Zhou, S.; Gao, H.; Lu, Z.; Wu, X.; Li, R.; Qiu, L.; Cai, Y.; Yao, Z., Sulfonamide antibiotics in the Northern Yellow Sea are related to resistant bacteria: implications for antibiotic resistance genes. Mar Pollut Bull 2014, 84, (1-2), 70-5.

472. Leonard, A. F. C.; Zhang, L. H.; Balfour, A.; Garside, R.; Gaze, W. H., Human recreational exposure to antibiotic resistant bacteria in coastal bathing waters. Environ Int 2015, 82, 92-100. 
473. Zhang, X. X.; Zhang, T.; Fang, H. H., Antibiotic resistance genes in water environment. Appl Microbiol Biotechnol 2009, 82, (3), 397-414.

474. Penders, J.; Stobberingh, E. E.; Savelkoul, P. H.; Wolffs, P. F., The human microbiome as a reservoir of antimicrobial resistance. Front. Microbiol. 2013, 4, 87.

475. Palumbi, S. R., Evolution - Humans as the world's greatest evolutionary force. Science 2001, 293, (5536), 1786-1790.

476. Clatworthy, A. E.; Pierson, E.; Hung, D. T., Targeting virulence: a new paradigm for antimicrobial therapy. Nat. Chem. Biol. 2007, 3, (9), 541-8.

477. Walker, A. W.; Duncan, S. H.; Louis, P.; Flint, H. J., Phylogeny, culturing, and metagenomics of the human gut microbiota. Trends Microbiol 2014, 22, (5), 267-74.

478. Band, V. I.; Crispell, E. K.; Napier, B. A.; Herrera, C. M.; Tharp, G. K.; Vavikolanu, K.; Pohl, J.; Read, T. D.; Bosinger, S. E.; Trent, M. S.; Burd, E. M.; Weiss, D. S., Antibiotic failure mediated by a resistant subpopulation in Enterobacter cloacae. 2016, 1, 16053.

479. Modi, S. R.; Lee, H. H.; Spina, C. S.; Collins, J. J., Antibiotic treatment expands the resistance reservoir and ecological network of the phage metagenome. Nature 2013, 499, (7457), 219-22.

480. Rinke, C.; Schwientek, P.; Sczyrba, A.; Ivanova, N. N.; Anderson, I. J.; Cheng, J. F.; Darling, A.; Malfatti, S.; Swan, B. K.; Gies, E. A.; Dodsworth, J. A.; Hedlund, B. P.; Tsiamis, G.; Sievert, S. M.; Liu, W. T.; Eisen, J. A.; Hallam, S. J.; Kyrpides, N. C.; Stepanauskas, R.; Rubin, E. M.; Hugenholtz, P.; Woyke, T., Insights into the phylogeny and coding potential of microbial dark matter. Nature 2013, 499, (7459), 431-437.

481. Almeida, M.; Pop, M.; Le Chatelier, E.; Prifti, E.; Pons, N.; Ghozlane, A.; Ehrlich, D. S., Capturing the most wanted taxa through cross-sample correlations. Isme J 2016.

482. Lagier, J. C.; Armougom, F.; Million, M.; Hugon, P.; Pagnier, I.; Robert, C.; Bittar, F.; Fournous, G.; Gimenez, G.; Maraninchi, M.; Trape, J. F.; Koonin, E. V.; La Scola, B.; Raoult, D., Microbial culturomics: paradigm shift in the human gut microbiome study. Clin. Microbiol. Infect. 2012, 18, (12), 1185-93.

483. Brown, C. T.; Hug, L. A.; Thomas, B. C.; Sharon, I.; Castelle, C. J.; Singh, A.; Wilkins, M. J.; Wrighton, K. C.; Williams, K. H.; Banfield, J. F., Unusual biology across a group comprising more than $15 \%$ of domain Bacteria. Nature 2015, 523, (7559), 208-11.

484. Lynch, M. D.; Neufeld, J. D., Ecology and exploration of the rare biosphere. Nat. Rev. Microbiol. 2015.

485. Lowe, D., A plan for new antibiotics. Sci. Transl. Med. 2016.

486. Cars, O.; Hedin, A.; Heddini, A., The global need for effective antibiotics-moving towards concerted action. Drug resistance updates : reviews and commentaries in antimicrobial and anticancer chemotherapy 2011, 14, (2), 68-9.

487. Dellit, T. H.; Owens, R. C.; McGowan, J. E., Jr.; Gerding, D. N.; Weinstein, R. A.; Burke, J. P.; Huskins, W. C.; Paterson, D. L.; Fishman, N. O.; Carpenter, C. F.; Brennan, P. J.; Billeter, M.; Hooton, T. M.; Infectious Diseases Society of, A.; Society for Healthcare Epidemiology of, A., Infectious Diseases Society of America and the Society for Healthcare Epidemiology of America guidelines for developing an institutional program to enhance antimicrobial stewardship. Clin Infect Dis 2007, 44, (2), 159-77.

488. Morrill, H. J.; Caffrey, A. R.; Jump, R. L.; Dosa, D.; LaPlante, K. L., Antimicrobial Stewardship in LongTerm Care Facilities: A Call to Action. J. Am. Med. Dir. Assoc. 2016, 17, (2), 183 e1-16.

489. Elliot, K., Center for Global Development, Antibiotics on the Farm: Agriculture's Role in Drug Resistance. http://www.cgdev.org/ 2015.

490. Watkins, R. R.; Bonomo, R. A., Overview: Global and Local Impact of Antibiotic Resistance. Infect. Dis. Clin. North Am. 2016, 30, (2), 313-22.

491. Imamovic, L.; Sommer, M. O. A., Use of Collateral Sensitivity Networks to Design Drug Cycling Protocols That Avoid Resistance Development. Sci. Transl. Med. 2013, 5, (204). 



\section{Summary}

One of the major threats to human health in the $21^{\text {st }}$ century is the emergence of pathogenic bacteria that are resistant to multiple antibiotics, thereby limiting treatment options. An important route through which pathogens become resistant is via acquisition of resistance genes from environmental and human-associated bacteria. Yet, it is poorly understood to what extent and by what mechanisms these so-called reservoirs contribute to emerging resistance. Therefore, the work described in this thesis focussed on generating novel insights into different niches as sources of resistance, with a particular focus on the human gut microbiota as well as on microbial communities associated with marine sponges, especially because the latter have been described as one of the richest sources of bioactive secondary metabolites, including a broad range of antimicrobials. Cultivation-based methods were complemented with culture-independent approaches in order to study bacterial taxa that are not readily cultivated.

Using metatranscriptomics it was found that clinically relevant antibiotic resistance genes are expressed in a broad range of environmental niches including human, mouse and pig gut microbiota, sea bacterioplankton, a marine sponge, forest soil and sub-seafloor sediment. The diversity of resistance gene transcripts differed greatly per niche indicating that the environment contains a rich reservoir of functional resistance that could be accessible by pathogens. Even though resistance gene expression might be linked to the presence of natural antibiotics, we did not detect expression of the corresponding secondary metabolite biosynthesis clusters.

Thirty-one antibiotic-resistant bacteria, amongst which three belonging to potentially novel Flavobacteriaceae spp., were isolated from the Mediterranean sponges Aplysina aerophoba, Corticium candelabrum and Petrosia ficiformis. Isolates were identified in a high throughput manner by double-barcoded 165 rRNA gene amplicon sequencing. Furthermore, analysis of sponge tissue-derived bacterial biomass growing on agar media showed that many novel bacterial taxa can still be isolated by conventional cultivation methods. Genomic DNA from the 31 antibioticresistant bacteria was interrogated with respect to the presence of active resistance genes by functional metagenomics. In addition, we also screened metagenomic libraries prepared from DNA directly isolated from sponge tissue in order to circumvent the need for cultivation. In total, 37 unique resistance genes were 
identified, and the predicted gene products of 15 of these shared $<90 \%$ amino acid identity with known gene products. One resistance gene (b/apsV-1), which was classified into a new $\beta$-lactamase family, was found to be exclusive to the marinespecific genus Pseudovibrio. These findings raised questions as to the functional roles of these genes in sponges, but more importantly, the functionality of these genes in $E$. coli shows that they can potentially be harnessed by phylogenetically distinct bacteria in other environments, including human pathogens. As such, it is a wake-up call as to the significance of marine resistance reservoirs.

Pseudovibrio, a genus of $\alpha$-Proteobacteria, was studied in more detail by comparative genomics as it comprises bacteria that potentially play a role as sponge symbionts and marine hubs of antibiotics resistance. Based on gene content, members of the genus Pseudovibrio were found to cluster by sponge sampling location indicating geographic speciation. Furthermore, Pseudovibrio spp. isolated from sponges near the Spanish coast clustered by sponge, suggesting host-specific colonization or adaptation. Strong support for Pseudovibrio spp. forming symbiotic relations with sponges came from the presence of a plethora of (predicted) conserved symbiosis-related functions in their genomes.

A final study aimed to isolate novel antibiotic resistant reservoir species from the human gut microbiota using a targeted approach. Faecal samples from hospitalized patients that received Selective Digestive Decontamination (SDD), a prophylactic treatment with a cocktail of different antibiotics (tobramycin, polymyxin $\mathrm{E}$, amphotericin B and cefotaxime), were inoculated anaerobically on agar media, after which bacterial biomass was analysed by $16 \mathrm{~S}$ rRNA gene amplicon sequencing. Six novel taxa were identified that, based on their growth on media supplemented with the SDD antibiotics, could serve as clinically relevant reservoirs of antibiotic resistance. For one of these six taxa a member was obtained in pure culture by targeted isolation. The abundance of antibiotic resistant uncultivated taxa in the human gut microbiota warrants further research as to their potential roles in resistance dissemination.

In conclusion, this thesis provides deeper insights into different environmental niches as reservoirs of antibiotic resistance. The results can serve to prime and inspire future research. 



\section{Appendices}

Nederlandse samenvatting

Acknowledgements

About the author

List of publications

Co-author affiliations

194

VLAG training activities 


\section{Nederlandse Samenvatting}

Eén van de grootste bedreigingen voor de volksgezondheid in de $21^{\text {ste }}$ eeuw is de opkomst van pathogene bacteriën die resistent zijn tegen meerdere antibiotica, waardoor infecties moeilijker te behandelen worden. Deze pathogenen kunnen onder andere resistent worden door het overnemen van resistentiegenen van bacteriën uit het milieu of van bacteriën die zich van nature met mensen associëren. Het is grotendeels nog onduidelijk in welke mate en via welke mechanismen deze zogenoemde resistentie reservoirs bijdragen aan de opkomende resistentie in pathogenen. Om die reden heeft het onderzoek in dit proefschrift zich gericht op het verkrijgen van nieuwe inzichten met betrekking tot de rol van verschillende resistentie reservoirs in de resistentieproblematiek, waarbij de nadruk is gelegd op de microbiota in menselijke darmen en sponsdieren. Bacteriën in sponsdieren zijn onderzocht omdat ze een grote verscheidenheid aan bioactieve stoffen kunnen produceren waaronder ook stoffen met een antibacteriële werking. Onderzoeksmethodieken die afhankelijk zijn van bacteriële cultivatie, zijn gecomplementeerd met cultivatieonafhankelijke methodieken, zodat we ook resistentie kunnen bestuderen in bacteriën die we nog niet kunnen cultiveren.

Met behulp van een metatranscriptome analyse was ontdekt dat klinisch relevante antibiotica-resistentiegenen tot expressie komen in een grote verscheidenheid aan ecologische niches waaronder de microbiota in de darmen van mensen, muizen en varkens, plankton in de zee en bacteriën in een sponsdier, bosgrond en sediment in de zeebodem. We vonden dat per niche verschillende resistentiegenen tot expressie kwamen, wat aangeeft dat het milieu een rijk reservoir is van functionele resistentiegenen waar pathogenen mogelijk een beroep op kunnen doen. We onderzochten ook of de expressie van resistentiegenen kon worden gelinkt aan de lokale productie van antibiotica aan de hand van de expressie van relevante biosynthese clusters, maar hiervoor werd geen bewijs gevonden.

Eenendertig antibioticaresistente bacteriën waren geïsoleerd uit de Mediterrane sponsdieren Aplysina aerophoba, Corticium candelabrum en Petrosia ficiformis. Hieronder zaten drie stammen die mogelijk nieuwe soorten zijn binnen de familie van Flavobacteriaceae. De 31 isolaten waren verkregen uit een groot cultivatie experiment waarin de bacteriën werden geïdentificeerd door middel van high throughput $16 \mathrm{~S}$ rRNA amplicon onderzoek met dubbele barcodes. Op basis van 
een analyse van bacteriële groei vanuit spons samples op agar kweekmedium werd geconcludeerd dat veel nieuwe bacteriesoorten nog steeds gecultiveerd kunnen worden met conventionele methodieken. Functionele metagenomics werd toegepast om te onderzoeken of de genomen van de 31 antibioticaresistente spons bacteriën functionele resistentiegenen bevatten. We onderzochten met deze methodiek ook DNA dat was geïsoleerd uit sponsdieren, teneinde de noodzaak van cultivatie te omzeilen. In totaal werden 37 unieke resistentiegenen geïdentificeerd en de bijbehorende eiwitsequenties van 15 hiervan deelden $<90 \%$ aminozuur identiteit met bekende eiwitten. Eén resistentiegen (blapsv-1) werd geclassificeerd in een nieuwe familie die tot zover alleen is gevonden in Pseudovibrio. Leden van het bacteriële genus Pseudovibrio vindt men exclusief in aquatische zoutwateromgevingen en ze worden veelvuldig geïsoleerd uit sponsdieren. Deze vindingen roepen vragen op wat betreft de functie van resistentiegenen in sponzen, maar belangrijker is dat de functionaliteit van deze genen in $E$. coli aantoont dat ze ingezet kunnen worden door bacteriën in andere taxa en dus ook pathogenen. Derhalve zijn deze resultaten een wekroep voor wat betreft de significantie van marine resistentie reservoirs.

Het genus Pseudovibrio was bestudeerd in detail in dit proefschrift door de genoominformatie van 31 Pseudovibrio stammen te vergelijken. De hypothese was dat Pseudovibrio symbionten zijn van sponsdieren en tevens was er een vermoeden dat ze dienen als hub voor de verspreiding van antibiotica resistentie. Er werd geconcludeerd dat leden van dit genus meer genetische overeenkomsten vertoonden wanneer ze waren geïsoleerd op dezelfde geografische locatie. Dit duidt op geografische speciatie. Er waren ook aanwijzingen voor spons-specifieke kolonisatie of adaptatie doordat Pseudovibrio stammen in een gebied nabij de Spaanse kust meer overeenkomsten vertoonden wanneer ze waren geïsoleerd uit dezelfde sponssoort. Veel steun voor de hypothese dat Pseudovibrio symbionten zijn van sponzen werd geleverd doordat de genomen codeerden voor een veelzijdigheid aan symbiose gerelateerde genen die ook nog eens sterk geconserveerd waren.

Het doel van de laatste studie was om door middel van een gerichte aanpak nieuwe soorten bacteriën uit de menselijke darmen te isoleren die zouden kunnen dienen als reservoirs voor antibiotica resistentie. Daarvoor werden monsters van faeces van intensive care patiënten die profylactische antibiotica (tobramycine, polymyxine E, amphotericine B en cefotaxime) toegediend kregen anaerobisch uitgeplaat op agar kweekmedia, waarna de bacteriële groei werd geanalyseerd 
middels 16S rRNA gen amplicon sequenties. Er werden zes nieuwe bacteriële taxa geïdentificeerd die groeiden op agar kweekmedia waaraan de profylactische antibiotica waren toegevoegd en zodoende zouden deze bacteriën kunnen dienen als klinisch relevante resistentie reservoirs. Een lid van één van deze zes taxa werd vervolgens gericht geïsoleerd. De grote hoeveelheid antibioticaresistente taxa in de menselijke darmen, waarvan nog geen leden zijn gecultiveerd, verantwoordt vervolgonderzoek naar hun mogelijke rol in de verspreiding van antibiotica resistentie.

Samenvattend, de kennis besloten in dit proefschrift verschaft nieuwe inzichten over de rol van verschillende ecologisch niches als antibiotica resistentie reservoirs. De resultaten kunnen dienen als leidraad en inspiratie voor vervolgonderzoek. 


\section{Acknowledgements}

The first step to finishing your PhD is getting lucky with your supervision, and that is definitely something I did very well. The contribution of my supervisors to the work described in this thesis is tremendous. First off, I would like to acknowledge my promotor Hauke, who thankfully gave me the opportunity to start as a PhD student in the MolEco group. Hauke, thanks for your continuous critical feedback and patience. Your dedication to us preparing quality manuscripts and doing quality research is appreciated. Next, I would like to acknowledge Mark, the man in charge of my daily supervision. Mark, I believe that your high standards in combination with your honest critiques were key to my development as a scientist. The high interest that you showed in any result or every bit of progress was motivating, and I would like to think that some of your high energy level and passion for science have rubbed off on me. Thanks. Finally, Detmer, thanks for your supervision and the opportunities you provided when my research focus started shifting from antibiotic resistance to sponges. Your curiosity and pragmatic approach towards scientific hurdles ensured our steady progress.

I would like to acknowledge all the people from the Laboratory of Microbiology and SSB (we don't want to forget anybody), and particularly the Molecular Ecology group. I am glad for your great company during breaks and at the many social activities (lab trips, Christmas dinners, MolEco cakes etc.), but also for our discussions in the lab or at MolEco and PhD meetings.

I also want to thank all the internal and external collaborators for their valuable contributions to the work in this thesis. Teresita, it was great to have another $\mathrm{PhD}$ student around who focussed exclusively on antibiotic resistance. Thanks for your valuable input during our many meetings, and our collaboration on the MicroDish study. My gratitude also goes out to Erwin for his insights into studies and associated methods of the human gut microbiota. Naim and Kyle, we did some good work studying sponge microbiota. Willem, thanks for our collaboration on the metatranscriptomics story amongst other stories. Elena, our collaboration on the analysis of the fosmid inserts was interesting as well as fruitful. Colin, Zalan and Petra, thanks for our discussions on the application of the MicroDish Chip. Morten, thanks for allowing me to visit your lab at the DTU and our collaboration. Mari, thanks for teaching me how to prepare small-insert libraries, and more importantly, for all the 
delicious lunches that you prepared and shared with me. Marco, your involvement in finishing the metatranscriptomics story was hugely appreciated. When it comes to 16S rRNA gene amplicon analysis, I would like to give a shout out to Javier for answering all my questions. Bart, I am happy for our successful collaboration on the Pseudovibrio comparative genomics story, thanks. Other people who discussed bioinformatics and statistical analyses with me, and whom I would like to acknowledge are: Jasper, Bastian, Peter, Edoardo, Lennart, Gerben and Romy.

Over the years there were many people that I depended on for carrying out wet lab work. Ineke, thanks for ordering countless batches of primers and loads of other lab materials. Steven and Monica, your instructions in the lab were much appreciated, especially with respect to helping me prepare for practicals. And Sjoerd, thanks for your insights into DNA manipulation. Other people that I would like to have mentioned are Sjon, Wilma, Jorn, Hans, Philippe and Ton.

As far as I could tell, the Laboratory of Microbiology always kept running smoothly. Let me identify the key players. Willem de Vos, thanks for creating a stimulating environment (and also your input on my work, mostly at PhD meetings). Anja and Carolien, thank you both for helping me arrange all kinds of bureaucratic (but necessary) stuff. Wim, thanks for your ICT and technical support.

I am happy to have supervised Tu, Steven and $\mathrm{Nga}$, who through their questions and results have also contributed to the work in this thesis.

I think that the word "sponge" in the thesis title gives away that I am also a proud member of the sponge team. I would like to thank the sponge people, namely Indra, Naim, Jie, Kyle, Johanna, Dat, João, Georg and Tika, for our discussions and their company. Naim, it was my pleasure being your paranymph.

I would like to thank all my office mates for creating a nice working atmosphere. My initial office mates were Thomas, Farai and Corina, to be followed by Tian, Yanling, Lennart, Indra, Peng Peng and Jie, and Ying, Kees, Georg and Hikmah in the Helix building. I would like to especially acknowledge Thomas for sharing his wisdom on a broad range of topics but especially on the politics of science.

Indra, it is an honour that you are my paranymph. I enjoyed our fun times in the office, and I am thankful that you always listened when I needed to complain about something. Clearly our silver medal shows that we formed an amazing badminton duo. I wish you the best of luck. 
Lennart, I am also honoured to have you as a paranymph at my side at a time when you yourself are at the last stretch of your $\mathrm{PhD}$. Our swimming sessions were a welcome and fun distraction from work. I was impressed by your eye for detail, both with respect to sports and science, and I can say that my front crawl will never be the same again. Good luck finishing your PhD.

Buiten het werk zijn er naaste familie en vrienden die hier niet genoemd worden, maar waar ik enorm dankbaar voor ben. LooWee, bedankt voor al je liefde en steun en tevens je begrip in perioden waarin alles leek te draaien om het proefschrift. De meeste dank ben ik verschuldigd aan mijn ouders, op wie ik al mijn hele leven onvoorwaardelijk kan rekenen. Zonder jullie had dit proefschrift er nooit gekomen.

Dennis Versluis 


\section{About the author}

Dennis Versluis was born on the $9^{\text {th }}$ of May, 1983, in Gorinchem, The Netherlands. He received his primary education at the public school CBS De Regenboog in Gorinchem, followed by pre-university secondary education at lyceum Oude Hoven in the same town. In 2001, he enrolled at Wageningen University, Wageningen, into the Biotechnology programme of which the MSc Biotechnology was awarded on March $13^{\text {th }}, 2008$, with Medical Biotechnology as specialisation.

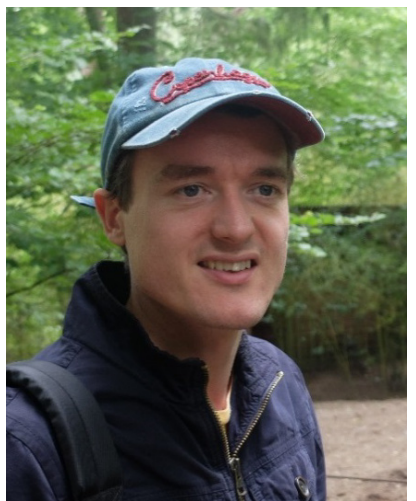
Next, he pursued the MSc of Neurosciences at Vrije Universiteit Amsterdam, which was awarded on August 31 $1^{\text {st }}, 2011$.

In February 2012, he started his $\mathrm{PhD}$ research at the Laboratory of Microbiology, Wageningen University, under the supervision of Prof. Dr. Hauke Smidt, Dr. Mark W.J. van Passel and Dr. Detmer Sipkema. The PhD research, which is described in this thesis, investigated antibiotic resistance reservoirs with a particular focus on the sponge microbiota and the human gut microbiota. In November 2016, he began working as a postdoctoral researcher at the Environmental Biochemistry group led by Prof. Dr. Peter Schupp at the Institute for Chemistry and Biology of the Marine Environment - Terramare, Wilhelmshaven, Germany. There, his studies focus on bioactivity and ecological roles of microorganisms associated with marine invertebrates. 


\section{List of publications}

Buelow E., Bello González T.deJ., Versluis D., Oostdijk E.A., Ogilvie L.A., van Mourik M.S., Oosterink E., van Passel M.W.J., Smidt H., D'Andrea M.M., de Been M., Jones B.V., Willems R.J., Bonten M.J., van Schaik W.. 2014. Effects of selective digestive decontamination $(S D D)$ on the gut resistome. J Antimicrob Chemother 69:2215-2223.

Versluis D, D'Andrea M.M., Ramiro Garcia J., Leimena M.M., Hugenholtz F., Zhang J., Ozturk B., Nylund L., Sipkema D., van Schaik W., de Vos W.M., Kleerebezem M., Smidt H., van Passel M.W.J.. 2015. Mining microbial metatranscriptomes for expression of antibiotic resistance genes under natural conditions. Sci. Rep. 5:11981.

Versluis D, McPherson K., van Passel M.W.J., Smidt H., Sipkema D.. 2016. High throughput recovery of previously uncultured bacterial genera from three Mediterranean sponges. Submitted to Marine Biotechnology.

Versluis D, de Evgrafov M.R., Sommer M.O.A., Sipkema D., Smidt H., van Passel M.W.J.. 2016. Functional metagenomics confirm sponge microbiota as a reservoir of antibiotic resistance genes. Submitted to Frontiers in Microbiology.

Versluis D, Nijsse, B.. Naim M.A., Koehorst J., Hornung B., Xu Y., Li Q., Wiese J., Zhang Y., Imhoff J.F., Schaap P., van Passel M.W.J., Smidt H., Sipkema D.. Comparative genomics of the marine genus Pseudovibrio highlights symbiotic capacities of versatile bacteria. To be submitted to Genome Biology and Evolution.

Versluis D, Bello González T.deJ., Zoetendal E.G, van Passel M.W.J., Smidt H.. High throughput cultivation-based screening on the MicroDish platform allows targeted isolation of antibiotic resistant human gut bacteria. In preparation. 


\section{Co-author affiliations}

Marco Maria D'Andrea ${ }^{1}$

Teresita de J. Bello González ${ }^{2}$

Mark de Been ${ }^{3}$

Marc J.M. Bonten ${ }^{3}$

Elena Bülow ${ }^{3}$

Bastian Hornung ${ }^{4}$

Floor Hugenholtz ${ }^{2,5,6}$

Johannes F. Imhoff ${ }^{7}$

Brian V. Jones ${ }^{8,9}$

Michiel Kleerebezem ${ }^{2,5,10,11}$

Jasper Koehorst ${ }^{4}$

Milkha M. Leimena ${ }^{2,5}$

Qi Li ${ }^{12}$

Kyle McPherson ${ }^{2}$

Maaike S.M. van Mourik ${ }^{3}$

Mohd Azrul Naim²

Bart Nijsse ${ }^{2,4}$

Lotta Nylund ${ }^{13,14}$

Lesley A. Ogilvie 8,15

Evelien A.N. Oostdijk ${ }^{3}$

Els Oosterink ${ }^{3}$

Başak Öztürk ${ }^{2}$

Mark W.J. van Passel ${ }^{2,16}$

Javier Ramiro Garcia ${ }^{2,5}$

Mari Rodriguez de Evgrafov ${ }^{17}$

Peter Schaap ${ }^{2}$

Willem van Schaik ${ }^{3}$

Detmer Sipkema ${ }^{2}$

Hauke Smidt ${ }^{3,5}$

Morten O.A. Sommer ${ }^{17}$

Willem M. de Vos $2,14,18$

Jutta Wiese $^{7}$

Rob J.L. Willems ${ }^{3}$

Ying $\mathrm{Xu}^{12,19}$

Jing Zhang ${ }^{2}$

Yu Zhang ${ }^{19}$

Erwin G. Zoetendal ${ }^{2}$

${ }^{1}$ Department of Medical Biotechnologies, University of Siena, Italy

${ }^{2}$ Laboratory of Microbiology, Wageningen University, Wageningen, The Netherlands

${ }^{3}$ Department of Medical Microbiology, University Medical Center Utrecht, Utrecht,

The Netherlands 
${ }^{4}$ Laboratory of Systems and Synthetic Biology, Wageningen University, Wageningen, The Netherlands

${ }^{5}$ Top Institute Food and Nutrition, Wageningen, The Netherlands

${ }^{6}$ Netherlands Consortium for Systems Biology, University of Amsterdam, The

Netherlands

${ }^{7}$ Marine Microbiology, GEOMAR Helmholtz Centre for Ocean Research Kiel, Kiel, Germany

${ }^{8}$ Center for Biomedical and Health Science Research, School of Pharmacy and Biomolecular Sciences, University of Brighton, Brighton, United Kingdom ${ }^{9}$ Queen Victoria Hospital NHS Foundation Trust, East Grinstead, United Kingdom

${ }^{10} \mathrm{NIZO}$ Food Research B.V., Ede, The Netherlands

${ }^{11}$ Host-Microbe Interactomics Group, Wageningen University, Wageningen, The Netherlands

${ }^{12}$ Division of Life Science, The Hong Kong University of Science and Technology, Hong Kong SAR, China

${ }^{13}$ Functional Foods Forum, University of Turku, Turku, Finland

${ }^{14}$ Department of Veterinary Biosciences, University of Helsinki, Finland

${ }^{15}$ Department of Vertebrate Genomics, Max Planck Institute for Infection Biology, Berlin, Germany

${ }^{16}$ National Institute for Public Health and the Environment, Bilthoven, The Netherlands

${ }^{17}$ Novo Nordisk Foundation Center for Biosustainability, Technical University of Denmark, Hørsholm, Denmark

${ }^{18}$ Department of Bacteriology and Immunology, Haartman Institute, University of Helsinki, Finland

${ }^{19}$ Shenzhen Key Laboratory of Marine Bioresource \& Eco-environmental Science, College of Life Science, Shenzhen University, Shenzhen, China 


\section{Overview of completed training activities}

\section{Discipline specific activities}

\section{Meetings}

- EvotAR annual meeting (oral presentation), Paris, FR

- NVvM spring meeting (oral presentation), Papendal, NL

- FEMS Microbiology Congress (poster presentation),

- EvoTAR annual meeting (oral presentation), Madrid, ES 2013

- NVvM spring meeting (2 oral presentations), Papendal, NL 2014

- EvoTAR annual meeting (oral presentation), Copenhagen, DK 2015

- NVvM spring meeting (poster presentation), Papendal, NL 2015

- ICETAR2015 (poster presentation), Amsterdam, NL 2015

Courses

- NBIC Metagenomics approaches and data analysis 2013 (oral presentation), Leiden, NL

- Rubicon ARB/SILVA basic training, Wageningen, NL 2014

- MolMed Adobe Photopshop \& Illustrator CS6 Workshop, 2014 Rotterdam, NL

\section{General courses}

- VLAG PhD week, Baarlo, NL 2012

- PhD Competence Assessment, Wageningen, NL 2012

- WGS Interpersonal Communication for PhD candidates, 2013 Wageningen, NL

- WGS Voice Matters - Voice and Presentation Skills Training, 2014 Wageningen, NL

- WGS Last Stretch of the PhD Programme, Wageningen, NL 2014

- WGS Career Perspectives, Wageningen, NL 2015

- BVO Assertiviteit op de Werkplek, Utrecht, NL 2015

- WGS Scientific Writing, Wageningen, NL

\section{Optional activities}

- Advanced bioinformatics (BIF-30806) 2012

- Preparation of research proposal 2012

- Laboratory of Microbiology PhD trip 2013

- Laboratory of Microbiology PhD meetings 2012-2016

- Molecular Ecology group meetings 2012-2016 
The research described in this thesis was financially supported by the European Commission's Seventh Framework Programme under grant agreement HEALTH.2011.2.3.1-2 (EvoTAR), coordinated by R.J. Willems (University Medical Center Utrecht).

Cover: abstract water

Printed by: GVO drukkers \& vormgevers, Ede, The Netherlands

Financial support from the Laboratory of Microbiology, Wageningen University, for printing of this thesis is gratefully acknowledged 
